Universidad deValladolid

PROGRAMA DE DOCTORADO EN INGENIERÍA INDUSTRIAL

TESIS DOCTORAL:

\title{
Desarrollo de un modelo de simulación energía-economía-medio ambiente basado en dinámica de sistemas
}

\author{
Presentada por Ignacio de Blas Sanz \\ para optar al grado de
}

Doctor por la Universidad de Valladolid

Dirigida por:

Dr. Luis Javier Miguel González

Dr. Carlos de Castro Carranza 

Universidad deValladolid

PROGRAMA DE DOCTORADO EN INGENIERÍA INDUSTRIAL

TESIS DOCTORAL:

\title{
Desarrollo de un modelo de simulación energía-economía-medio ambiente basado en dinámica de sistemas
}

\author{
Presentada por Ignacio de Blas Sanz \\ para optar al grado de
}

Doctor por la Universidad de Valladolid

Dirigida por:

Dr. Luis Javier Miguel González

Dr. Carlos de Castro Carranza 

Esta tesis doctoral se ha desarrollado parcialmente en el marco de los proyectos europeos MEDEAS y LOCOMOTION financiados por el programa de investigación e innovación Horizonte 2020 de la Unión Europea en virtud de los acuerdos de subvención 691287 y 821105 respectivamente. El autor agradece también el apoyo del proyecto MODESLOW financiado por el Ministerio de Economía y Competitividad de España. (ECO2017-85110-R). 



\section{Resumen}

Los modelos de energía-economía-medio ambiente, o modelos de evaluación integrada (IAM), surgen con el objetivo de ayudar a analizar las complejas relaciones existentes entre las cuestiones socioeconómicas y las medioambientales, e influir en la toma de decisiones para reducir los impactos que la actividad humana está causando sobre el medio ambiente. El desarrollo de estos modelos, cada vez más utilizados por las principales instituciones, es clave para poder analizar la sostenibilidad y conseguir preservar las condiciones ambientales del planeta.

Esta tesis doctoral muestra el desarrollo completo de un modelo de energía-economía-medio ambiente, comenzando con una revisión previa de la literatura para buscar las limitaciones existentes en otros modelos y finalizando con un ejemplo de aplicación del propio modelo. El modelo, llamado MEDEAS-W debido al proyecto europeo homónimo dentro del cual se ha desarrollado, añade características que, por lo general, no están incorporadas en otros modelos. Algunas de las nuevas características son: la representación de las limitaciones biofísicas a la disponibilidad de energía y su realimentación sobre la economía, el modelado de los requerimientos energéticos y materiales para la transición energética, la integración de la estructura económica a través de tablas input-output o la realimentación de los daños del cambio climático en las variables socioeconómicas.

Las potencialidades del modelo han sido mostradas mediante simulaciones de escenarios continuistas en las que se obtienen que, en ausencia de restricciones energéticas y de daños causados por el cambio climático, el PIB y las emisiones de gas efecto invernadero (GEI) crecerán durante todo el siglo XXI generando un aumento de la temperatura global del planeta de más de $3^{\circ} \mathrm{C}$ respecto a los valores preindustriales. $\mathrm{Al}$ activar una de las restricciones anteriores, o ambas conjuntamente, los resultados se modifican completamente y el modelo muestra una recesión económica persistente durante las próximas décadas.

El modelo MEDEAS-W también ha permitido estudiar la descarbonización del sector transporte a nivel mundial. Este análisis muestra, por ejemplo, que las tendencias actuales en la descarbonización del sector transporte están muy lejos de poder alcanzar los objetivos de reducción de emisiones de GEI planteados por las principales instituciones, y que el uso masivo de vehículos eléctricos encuentra importantes problemas de disponibilidad en los minerales clave como son el litio, el cobre o el manganeso. Las simulaciones del modelo nos señalan que para poder alcanzar los principales objetivos de descarbonización es necesaria la aplicación de políticas radicales de sustitución de vehículos convencionales por vehículos eléctricos muy ligeros como bicicletas y ciclomoteres, junto con cambios importantes en los modos de comportamiento de los ciudadanos.

Por último, y aunque MEDEAS-W es una herramienta que permite analizar la sostenibilidad global y evaluar las políticas que se pueden llevar a cabo con el objetivo de evitar el deterioro medioambiental del planeta, en esta tesis se muestran algunas limitaciones del modelo encontradas durante su proceso de validación y se señalan futuras líneas de trabajo. 


\begin{abstract}
Energy-economy-environment models, or integrated assessment models (IAMs), arise with the aim of helping to analyze the complex relationships between socio-economic and environmental issues, and influence decision-making to reduce impacts that human activity is causing on the environment. The development of these models, increasingly used by the leading institutions, is the key to analyzing sustainability and preserving the planet's environmental conditions.

This doctoral thesis shows the complete development of an energy-economy-environment model, beginning with a previous literature review in order to explore existing limitations in other models and ending with an example of the application of the model. The model, called MEDEAS-W, due to the homonymous European project within which it was developed, adds features that are generally not incorporated in other models. Some of the new features are: the representation of the biophysical limitations to the availability of energy and its feedback on the economy, the modeling of the energy and material requirements for the energy transition, the integration of the economic structure through input-output tables, or the feedback of the damages of climate change in the socioeconomic variables.
\end{abstract}

The potential of the model has been shown through simulations of BAU scenario in which it is obtained that, in the absence of energy restrictions and damage caused by climate change, GDP and greenhouse gas (GHG) emissions will be growing throughout the XXI century generating an increase in the global temperature of the planet of more than $3{ }^{\circ} \mathrm{C}$. By activating one of the restrictions, or both together, the results completely modify and the model shows a persistent economic recession for the next few decades.

The MEDEAS-W model has also helped to study the decarbonisation of the world transportation sector. This analysis shows, for example, that current trends in the decarbonisation of the transportation sector are far from being able to achieve the GHG emission reduction targets set by the leading institutions, and that the massive use of electric vehicles faces significant availability problems in key minerals such as lithium, copper or manganese. The model simulations show us that in order to achieve the main decarbonisation objectives, it is necessary to apply radical policies to replace conventional vehicles with very light electric vehicles such as bicycles and mopeds, together with important changes in the behavior of citizens.

Finally, and although MEDEAS-W is a tool that allows to analyze global sustainability and evaluate the policies that can be carried out with the aim of avoiding the environmental deterioration of the planet, this doctoral thesis shows certain limitations of the model found during its process of validation and possible further lines of work are pointed out. 


\section{Agradecimientos}

La realización de esta tesis doctoral ha sido posible gracias a la ayuda de muchas personas y es por ello que me gustaría agradecer:

A mis supervisores Epi y Carlos, por su confianza en mí desde el principio, por sus aportaciones, consejos, ayuda y ánimos para que esta larga tarea haya llegado a su fin.

A todos los miembros del GEEDS de la Universidad de Valladolid, ya que esta tesis en cierta medida es fruto del trabajo de todos ellos, por todo lo que me han enseñado, por su paciencia con cada una de las cuestiones que les planteaba, por su cercanía y por su apoyo. En particular, a Iñigo, Jaime, David y Marga por su infatigable ayuda, por su generosidad y por haber hecho que esta aventura se haya convertido en una gran experiencia a su lado. También agradecer a la comunidad universitaria de la Universidad de Valladolid que me ha acompañado y ayudado durante todo el doctorado, en especial al Departamento de Ingeniería de Sistemas y Automática de la Escuela de Ingenieros Industriales, y a Yania y al resto de profesores con los que he trabajado de la Escuela de Ingeniera Informática.

A los socios de los proyectos europeos con los que he estado trabajando durante estos años, por todos los conocimientos que me han transmitido acerca de la investigación científica y la importancia del trabajo colectivo. En especial a Roger, Antonio y Jordi del CSIC-Instituto de Ciencias del Mar de Barcelona por lo bien que me acogieron y todo lo que me enseñaron durante mi estancia de varios meses en su institución.

Por último, dar las gracias a mis padres, mi hermana, Soraya y el resto de mi familia y amigos por su apoyo incondicional durante todos mis años de formación y sin el cual no habría podido llevar a cabo este proyecto. 
PARTE I: INTRODUCCIÓN Y REVISIÓN DE LITERATURA ......................................................1

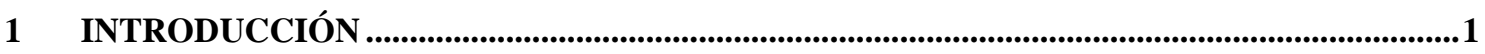

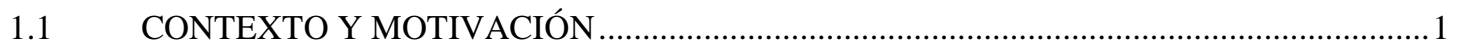

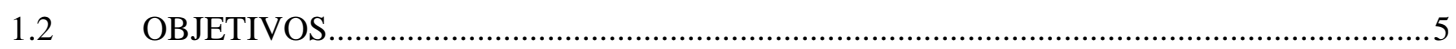

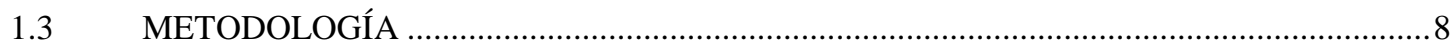

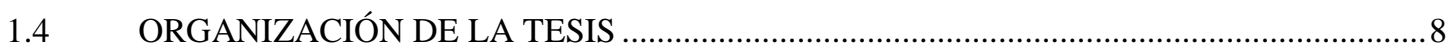

2 REVISIÓN DE MODELOS DE ENERGÍA-ECONOMÍA-MEDIO AMBIENTE (IAM) .........11 PARTE II: DESARROLLO DE UN MODELO DE ENERGÍA-ECONOMÍA-MEDIO AMBIENTE: MEDEAS.

3 MEDEAS: UN NUEVO MARCO DE MODELIZACIÓN QUE INTEGRA LAS LIMITACIONES BIOFÍSICAS Y SOCIOECONÓMICAS MUNDIALES .........................................12

4 MODELIZACIÓN DE LA DEMANDA ENERGÉTICA SECTORIAL A TRAVÉS DE LAS INTENSIDADES ENERGÉTICAS EN LOS MODELOS DE EVALUACIÓN INTEGRADA MEDEAS

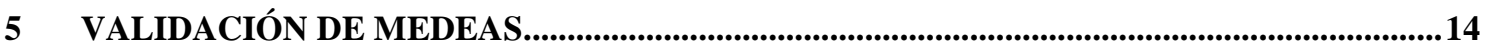

5.1 INTRODUCCIÓN A LA VALIDACIÓN DE MODELOS ....................................................

5.1.1 VALIDACIÓN DE MODELOS DE SIMULACIÓN …………………………....................

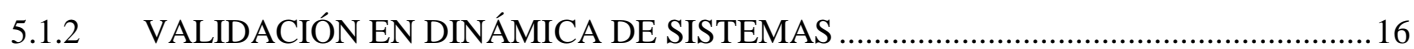

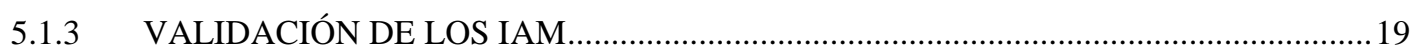

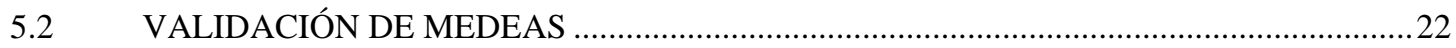

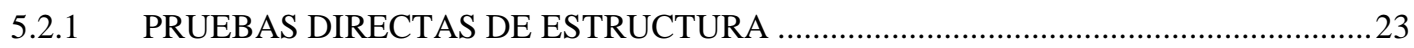

5.2.2 PRUEBAS INDIRECTAS DE ESTRUCTURA …………….........................................22

5.2.3 PRUEBAS DE PATRONES DE COMPORTAMIENTO_...................................................53

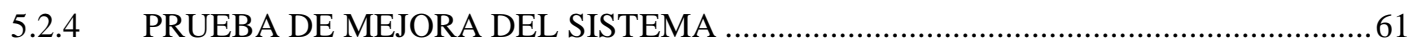

5.3 CONCLUSIONES DEL PROCESO DE VALIDACIÓN DE MEDEAS ……………...............62

PARTE III: APLICACIÓN DE MEDEAS: ANÁLISIS DE POLÍTICAS EN EL SECTOR

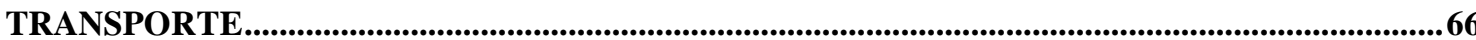

6 LOS LÍMITES DE LA DESCARBONIZACIÓN DEL TRANSPORTE EN EL ACTUAL PARADIGMA DE CRECIMIENTO.................................................................................................66

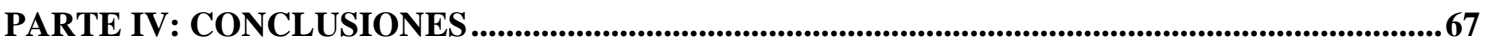

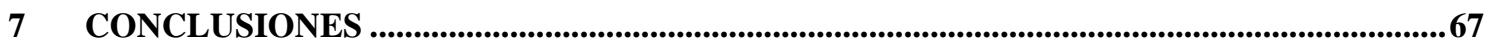

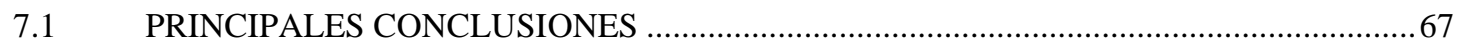

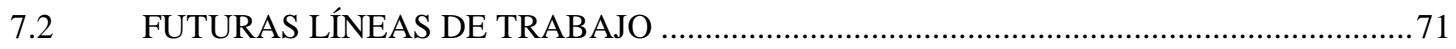

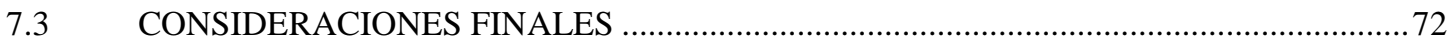

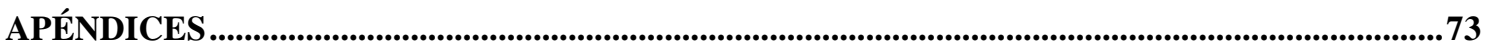

APPENDIX A: MATERIAL SUPLEMENTARIO DEL CAPÍTULO 3 …………………………........73

APPENDIX B: MATERIAL SUPLEMENTARIO DEL CAPÍTULO 4...................................................73

APPENDIX C: MATERIAL SUPLEMENTARIO DEL CAPÍTULO 6 ……………………………....73 
REFERENCIAS 


\section{Listado de tablas:}

Tabla 5.1: Ventajas y limitaciones de ocho métodos de evaluación de los IAM...................................221

Tabla 5.2: Tabla de los límites del módulo de la demanda de energía en MEDEAS-W v2.0 ..................27

Tabla 5.3: Valores del PIB y del aumento de la temperatura global en los años 1995, 2050 y 2100 utilizando

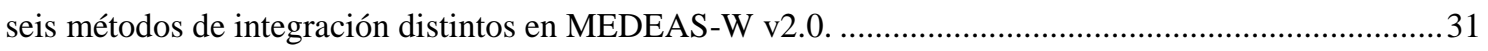
Tabla 5.4: Valores del PIB y del cambio en la temperatura global en los años 1995, 2050 y 2100 utilizando seis tiempos de paso distintos en MEDEAS-W v2.0 ...................................................................... 32 Tabla 5.5: Rangos de incertidumbre de las variable de entrada para el análisis de condiciones extremas en MEDEAS-W v2.0.

Tabla 5.6: Nombres de los parámetros exógenos en pymedeas_eu 0.3.0 utilizados para el análisis de sensibilidad junto con su descripción, sus unidades y sus valores nominales, mínimos y máximos..........40 Tabla 5.7: Nombres de los parámetros endógenos en pymedeas_eu 0.3.0 utilizados para el análisis de sensibilidad junto con su descripción, sus unidades, los índices afectados y sus valores mínimos y máximos.

Tabla 5.8: Lista de salidas del modelo MEDEAS-EU que se han utilizado en la prueba de análisis de sensibilidad.

Tabla 5.9: Rangos de incertidumbre de las variables de entrada para el análisis de incertidumbre realizado en los 4 cuatro casos de estudio de Capellán-Pérez et al., (Capellán-Pérez et al., 2020).. . .45 


\section{Listado de figuras:}

Figura 1.1: Estado actual de siete de los nueve límites planetarios. ........................................................4

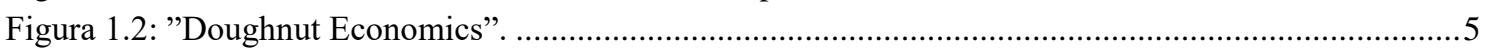

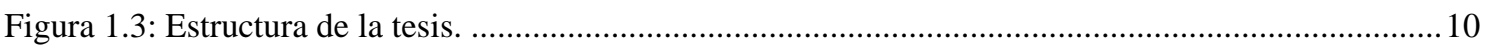

Figura 5.1: Versión simplificada del proceso de desarrollo de un modelo con sus fases de validación. .... 16 Figura 5.2: Secuencia lógica de las pruebas a realizar para la validación de un modelo de dinámica de

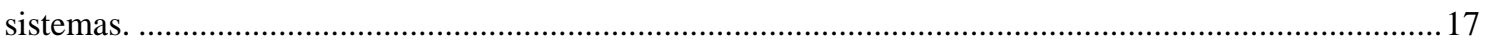

Figura 5.3: Secuencia lógica de desarrollo de un modelo con sus pruebas de validación. .......................... 18

Figura 5.4: Diagrama causal que muestra los efectos de la escasez de una energía final (i) en el marco de

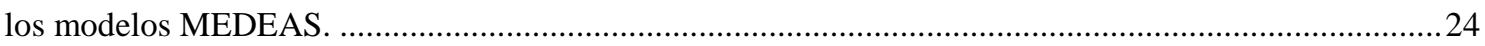

Figura 5.5: Diagrama causal que muestra las principales relaciones de las intensidades energéticas en el

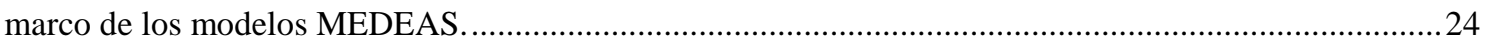

Figura 5.6: Ejemplo de bucle de primer orden. Variable de estado: "S" y flujo: "Net Inflow Rate". ........25 Figura 5.7: Parte del submódulo de las intensidades energéticas en MEDEAS-W v2.0 en el que se muestran los bucles de primer orden entre el stock y sus flujos de entrada y salida..........................................225

Figura 5.8: Diagrama de subsistema del módulo de la demanda de energía en MEDEAS-W..................28

Figura 5.9: Evolución del PIB a nivel mundial en billones de dólares de EEUU del año 1995 utilizando seis

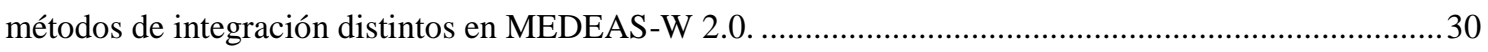

Figura 5.10: Cambio en la temperatura global utilizando seis métodos de integración distintos en MEDEAS-

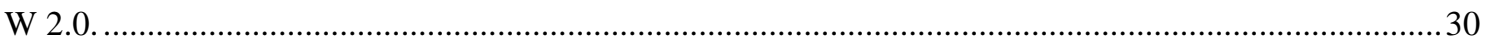

Figura 5.11: Evolución del PIB en billones de dólares de EEUU del año 1995 a nivel mundial utilizando seis tiempos de paso distintos en MEDEAS-W 2.0 ................................................................................ 31 Figura 5.12: Cambio en la temperatura global utilizando seis tiempos de paso distintos en MEDEAS-W 2.0.

Figura 5.13: Intervalos de confianza del PIB, del aumento de la temperatura global y del consumo total de energía final a nivel mundial bajo las condiciones extremas de la Tabla 5.5 en el modelo MEDEAS-W v2.0.

Figura 5.14: Intervalos de confianza de la parte de la energía cubierto por fuentes renovables, de la extracción total de petróleo y de la superficie de tierra necesaria para energías renovables a nivel mundial bajo las condiciones extremas de la Tabla 5.5 en el modelo MEDEAS-W v2.0.....................................3

Figura 5.15: Intervalos de confianza para el EROI estándar del sistema tras las 1000 simulaciones del análisis de Monte Carlo en el modelo MEDEAS-EU v1.2 ...................................................................38

Figura 5.16: Intervalos de confianza para las variables PIB, PIB per cápita, consumo final de energía total y emisiones totales de $\mathrm{CO}_{2}$ tras las 1000 simulaciones del análisis de Monte Carlo en el modelo MEDEAS-

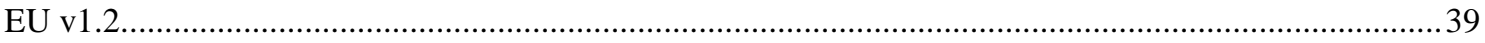
Figura 5.17: "Spider plots" en el año 2050 obtenidas en el análisis de sensibilidad realizado a pymedeas_eu

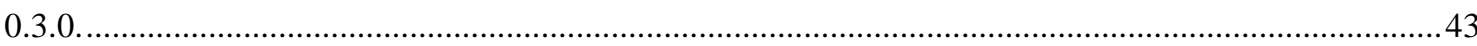

Figura 5.18: Distribución de probabilidades del consumo total de energía final per cápita en GJ por persona en MEDEAS-W v1.3.33, para los cuatro casos considerados en Capellán-Pérez et al (Capellán-Pérez et al., 2020).

Figura 5.19: Distribución de probabilidades de la parte de la energía primaria total cubierta por energías renovables en MEDEAS-W v1.3.33 para los cuatro casos considerados en Capellán-Pérez et al (CapellánPérez et al., 2020). .48

Figura 5.20: Distribución de probabilidades del producto interior bruto per cápita (PIBpc) en dólares de EEUU del año 1995 en MEDEAS-W v1.3.33, para los cuatro casos considerados en Capellán-Pérez et al (Capellán-Pérez et al., 2020).

Figura 5.21: Distribución de probabilidades de las emisiones totales de gases de efecto invernadero en gigatoneladas de $\mathrm{CO}_{2}$ equivalente en MEDEAS-W v1.3.33, para los cuatro casos considerados en CapellánPérez et al (Capellán-Pérez et al., 2020).

Figura 5.22: Distribución de probabilidades del aumento en la temperatura global en ${ }^{\circ} \mathrm{C}$ en MEDEAS-W v1.3.33, para los cuatro casos considerados en Capellán-Pérez et al (Capellán-Pérez et al., 2020)...........51 
Figura 5.23: Distribución de probabilidades de la intensidad de $\mathrm{CO}_{2}$ equivalente en la energía final en gigatoneladas de $\mathrm{CO}_{2}$ equivalente partido EJ en MEDEAS-W v1.3.33, para los cuatro casos considerados en Capellán-Pérez et al (Capellán-Pérez et al., 2020).

Figura 5.24: Ejemplos de síntomas que se obtienen en MEDEAS-W y muestran un deterioro ambiental del planeta en una simulación del escenario BAU.

Figura 5.25: Comparación entre el comportamiento obtenido en el modelo y el comportamiento histórico en las variables.

.55

Figura 5.26: Panel con la evolución de distintas variables para dos simulaciones en las que se activa/desactiva el bucle del EROI sobre la demanda de energía en MEDEAS-W v2.0.........................57 Figura 5.27: Evolución del PIB per cápita (dólares de EEUU de 1995/persona) y de las emisiones de $\mathrm{CO}_{2}$ (gigatoneladas de $\mathrm{CO}_{2}$ ), para cuatro modelos MEDEAS.

Figura 5.28: Panel con la evolución de distintas variables al mantener las tendencias actuales (línea roja) y en una situación de gran electrificación del transporte (línea verde) en MEDEAS-W v1.3.33. . .60 


\section{Abreviaturas y acrónimos}

- BAU: Business as usual

- BECCS: Bioenergy with carbon capture and sequestration

- BEV: Battery electric vehicles

- BP: British Petroleum

- CC: Climate change

- CCS: Carbon capture and sequestration

- CDR: carbon dioxide removal

- CEV: Catenary electric vehicles

- CHV: Catenary hybrid vehicles

- CNG: Compressed natural gas

- CSP: Concentrated solar power

- CTL: Coal to liquids

- Dmnl: Dimensionless.

- EEA: European Environment Agency

- EERE: Energy Efficiency and Renewable Energy

- EI: Energy intensity

- EOL: End-of-lifetime

- EROI: Energy return on energy investment

- ESOI: Energy stored on energy invested.

- EU: European Union. EU-28.

- EV: Electric vehicles

- EWG: Energy Watch Group

- FAO: Food and Agriculture Organization

- FE: Final energy

- FF: Forgetting factor

- GDP: Gross domestic product

- GDPpc: Gross domestic product per capita

- GEEDS: Grupo de energía, economía y dinámica de sistemas

- GEI: Gas de efecto invernadero

- GFCF: Gross fixed capital formation.

- GG: Green growth

- GHG: Greenhouse gas

- GTL: Gas to liquids

- HDI: Human Development Index

- HH: Households.

- HV: Heavy vehicles

- IAM: Integrated assessment model

- IAMC: Integrated assessment model consortium

- ICE: Internal combustion engines

- IEA: International Energy Agency

- IIASA: International Institute for Applied Systems Analysis

- iLUC: indirect land use change

- IOA: Input output analysis

- IOT: Input-Output tables.

- IPCC: Intergovernmental Panel on Climate Change

- IRENA: International Renewable Energy Agency

- LCA: Life-cycle analysis. 
- LNG: Liquefied natural gas

- LPG: Liquefied petroleum gas

- LV: Light vehicles

- MEDEAS-AUT: MEDEAS-Austria

- MEDEAS-BG: MEDEAS-Bulgaria

- MEDEAS-EU: MEDEAS-Europe

- MEDEAS-W: MEDEAS-World

- MIP: Model inter-comparison project

- NRE: Non-renewable energy sources

- MLP: Multi-Level Perspective

- ONU: Organización de Naciones Unidas

- O\&M: Operation and maintenance

- PIB: Producto interior bruto

- PHS: Pumped hydro storage.

- PS: Perception of scarcity

- PV: Photovoltaic

- RCP: Radiative concentration pathway

- RES: Renewable energy sources

- RK: Runge Kutta

- RMSD: Raíz del error cuadrático medio

- RTS: Reference technology scenario

- R\&D: Research and development

- SDG: Sustainable development goal

- SS: Sensitivity to scarcity

- SSP: Shared socioeconomic pathway

- TEV: Tracked electric vehicle

- TFEC: Total final energy consumption

- UN: United Nations

- UNEP: United Nations Environment Programme

- UNFCCC: United Nations Framework Convention on Climate Change

- URR: Ultimately Recoverable Resources

- WEO: World Energy Output

- WMO: World Meteorological organization

- WIOD: World Input-output database 


\section{PARTE I: INTRODUCCIÓN Y REVISIÓN DE LITERATURA}

\section{Introducción}

\subsection{Contexto y motivación}

La preocupación por la pérdida de la biodiversidad del planeta y su impacto sobre las estructuras socioeconómicas, debido especialmente a los efectos del cambio climático, es cada vez más importante para la comunidad científica, para las principales instituciones y para la sociedad en general. Una prueba de ello es que la presidenta de la Comisión Europea, Ursula von der Leyen, en su discurso de presentación ante la Comisión Europea, estableció como el principal objetivo de su mandato mantener el planeta sano (European Comission, 2019):

"Our most pressing challenge is keeping our planet healthy. This is the greatest responsibility and opportunity of our times. I want Europe to become the first climate-neutral continent in the world by 2050"

Las cuestiones medioambientales marcarán las políticas de los próximos años tanto a nivel europeo como a nivel mundial según los compromisos adquiridos en la cumbre del clima de París de 2015, donde una gran mayoría de países se comprometieron a reducir sus emisiones para evitar que el aumento de la temperatura global del planeta supere $\operatorname{los} 2^{\circ} \mathrm{C}$ (UNFCCC, 2015).

La preocupación por los cambios que el ser humano está generando sobre el planeta no es algo reciente. Hace más de 50 años, en el año 1968, científicos y políticos de distintos países fundaron el Club de Roma con el objetivo de mejorar el futuro a largo plazo de la humanidad y del planeta desde una perspectiva interdisciplinar y global. El Club de Roma publicó en el año 1972 el informe "Los límites del crecimiento", elaborado entre otros por Donella y Dennis Meadows, en el que mostraba las tendencias insostenibles a nivel mundial que se producían en los escenarios continuistas analizados en un modelo computacional elaborado por Jay Forrester (Meadows et al., 1972).

Años más tarde, en 1992, más de 1700 destacados científicos, entre los que se encontraban una gran parte de los premios Nobel en ciencias hasta la fecha, firmaron un documento llamado "Advertencia de los científicos del mundo a la humanidad" en el que señalaban que los seres humanos y el mundo natural están en camino a una colisión, que las actividades humanas infligen daños severos y a menudo irreversibles al medio ambiente y que si no se controlan estas actividades se está poniendo en grave riesgo el futuro de la sociedad humana y de los reinos vegetales y animales (UCS, 1992). En noviembre de 2017, 25 años después, más de 15000 científicos firmaron "Advertencia de los científicos del mundo a la humanidad: un segundo aviso" en el que planteaban que estamos poniendo en peligro nuestro futuro al no frenar nuestro intenso consumo de recursos (materiales, combustibles fósiles, alimentos, etc), no limitar adecuadamente el crecimiento demográfico, no reevaluar el papel de una economía basada en el crecimiento, no reducir los gases de efecto invernadero, no restaurar los ecosistemas, etc. (Ripple et al., 2017).

En las últimas décadas se han creado distintas organizaciones con el objetivo de luchar contra el deterioro que el ser humano está causando sobre el medio ambiente. Entre ellas destaca el "Grupo Intergubernamental de Expertos sobre el Cambio Climático de la ONU (IPCC)”. E1 IPCC publica informes periódicos sobre el papel del ser humano en el cambio climático (IPCC, 2018, 2014, 2007, 2001, 1992). Estos informes son utilizados en los principales tratados internacionales sobre cambio climático como el protocolo de Kyoto de 1997 (UN, 1998) o el acuerdo de París de 2015 (UNFCCC, 2015). La ONU también elaboró en el año 2015 los objetivos de desarrollo sostenible para el año 2030 (ODS) en los que plantea 17 objetivos a cumplir, entre los que se encuentran 
garantizar el acceso a una energía asequible, segura y sostenible para todos, conservar y utilizar de forma sostenible los océanos, luchar contra la desertificación o poner freno a la pérdida de diversidad biológica (UN, 2015a).

Sin embargo, pese a las advertencias realizadas por científicos y organizaciones, la humanidad no está tomando medidas urgentes para salvaguardar la salud del planeta. Los indicadores climáticos nos muestran una situación cada vez más preocupante. La concentración de $\mathrm{CO}_{2}$ en la atmósfera a finales del año 2020 ha alcanzado las 413 ppm en comparación con las 317 ppm del año 1960 (US Department of Commerce, 2020). Los últimos cinco años son los más cálidos de los que se tiene constancia, y la última década 2010-2019 es también la más cálida conocida (WMO, 2020). En 2019 el calentamiento global debido a la actividad humana alcanza aproximadamente $1,1 \pm 0,1^{\circ} \mathrm{C}$ por encima de los valores preindustriales (WMO, 2020). Este aumento de la temperatura se ve reflejado en el nivel del mar, que aumenta más de $3 \mathrm{~mm}$ por año, habiendo crecido casi $100 \mathrm{~mm}$ desde el año 1993, y el ritmo al que aumenta el nivel del mar se está acelerando cada vez más (NASA Global Climate Change, 2020).

Además, la destrucción y degradación de las reservas naturales y los ecosistemas por parte del ser humano es cada vez más evidente. En el período 1961-2013, la superficie de tierras desérticas ha aumentado más del 1\% de promedio anual, con una gran variabilidad interanual (IPCC, 2019). En 2015, alrededor de 500 millones de personas vivían dentro de zonas que se desertificaron en los últimos 40 años. Se estima que en la actualidad la erosión del suelo de los campos agrícolas es entre 10 y 100 veces más alta, dependiendo del tipo de cultivo, que la tasa de formación del suelo (IPCC, 2019). Por otra parte, se están derritiendo 335 mil millones de toneladas de hielo anuales, tres veces el volumen de todo el hielo acumulado en los Alpes, reduciéndose de una manera muy importante los glaciares del planeta (Zemp et al., 2019). La destrucción de las reservas naturales también ha llegado al reino animal. Desde el año 1993 se ha demostrado o se sospecha firmemente que se han extinguido 15 especies de aves y mamíferos y que actualmente el 23,7\% de las especies se encuentran en peligro de extinción (UN Environment, 2020).

Este deterioro ambiental del planeta se ha producido simultáneamente con un crecimiento exponencial de la población mundial desde los 4 mil millones de habitantes en 1975 a los más de 7,8 mil millones actuales (UN, 2020). La actividad económica prácticamente se ha triplicado en los últimos 30 años (World Bank, 2020) lo que junto al aumento de la población ha generado un crecimiento enorme en el consumo de recursos energéticos desde los 240 EJ en el año 1975 a los 583 EJ del año 2019 (BP, 2020). También se ha multiplicado el consumo de recursos minerales y de alimentos (FAO, 2020; IEA, 2019a). Cada vez son más los estudios que plantean que si no se cambian los patrones actuales de comportamiento será muy difícil mantener la sostenibilidad del planeta en el futuro (Alexander and Yacoumis, 2018; Cosme et al., 2017; Enríquez et al., 2020; Kerschner et al., 2018).

La defensa de la sostenibilidad del planeta es una necesidad para una gran mayoría de científicos y organizaciones especializadas. Existe una gran diversidad de definiciones de sostenibilidad. En 1997 la Agencia Europea del Medio Ambiente ya identificaba 300 interpretaciones diferentes (EEA, 1997). Una de las definiciones más influyentes es la que se encuentra en el informe "Our common future" de Naciones Unidas (UN, 1987). En él se define el desarrollo sostenible como aquel desarrollo que satisface las necesidades del presente sin comprometer la capacidad de las generaciones futuras para satisfacer sus propias necesidades. Esta definición tan general, y también tan antropocéntrica, está sujeta a múltiples interpretaciones (UN, 1987). Pese a esos muchos enfoques, la mayoría de definiciones de sostenibilidad están relacionadas con que: todo está sujeto a unos límites, es necesaria una distribución equitativa de los recursos y las 
oportunidades (tanto en términos regionales como temporales) y existe una interrelación entre la economía y la sociedad con el medio ambiente (Capellán-Pérez, 2016).

Desde que en el año 1972 el Club de Roma publicara el informe de "Los límites del crecimiento" (Meadows et al., 1972) ha existido un gran debate acerca de la posibilidad de poder mantener las tendencias actuales de comportamiento durante las próximas décadas y hasta qué punto este comportamiento puede modificar el planeta tal como lo conocemos. El informe de "Los límites del crecimiento" fue duramente criticado en los primeros años tras su publicación (Cole et al., 1976; Solow, 1973). Sin embargo, más de 30 años después se ha observado que los datos históricos se ajustan en gran medida a los obtenidos en el escenario base del informe (Turner, 2008). En la actualidad ese debate sigue existiendo, y aunque cada vez está más extendida la idea de que no es posible un crecimiento infinito dentro de un mundo finito, todavía una parte de la sociedad confía en que la capacidad de adaptación del ser humano y la mejora tecnológica permitan continuar con el comportamiento actual, aunque para ello se modifique una parte de los ecosistemas y las reservas naturales.

La realidad es que una gran parte de los recursos existentes en la biosfera ya han sido utilizados por la humanidad. A finales del siglo XX varios geólogos, siguiendo la estela de Hubbert (Hubbert, 1956), estimaron que el pico máximo de extracción de petróleo convencional se alcanzaría en algún momento de la primera década del siglo XXI (Campbell and Laherrère, 1998). Años después, en 2010, la Agencia Internacional de la Energía planteó que el pico máximo de extracción del petróleo convencional ya había pasado (IEA, 2010a). Los mismos fundamentos de la teoría del pico de Hubbert han permitido extender el concepto a otros combustibles no renovables, como el gas, el carbón y los combustibles fósiles no convencionales, planteando el pico de estos combustibles en la primera mitad del siglo XXI (Maggio and Cacciola, 2012; Mohr et al., 2015; Zittel et al., 2013). En el caso de los recursos energéticos renovables, estos tienen un potencial máximo que limita su producción total de energía (IPCC, 2012). Algunas energías renovables como la hidroelectricidad ya han utilizado una parte importante de su potencial máximo, sobre todo en Europa y Norteamérica (Gernaat et al., 2017). Otra metodología basada en la teoría del pico de Hubbert permite estudiar la disponibilidad de los recursos minerales, en ese estudio se obtiene que el ser humano ya ha utilizado una gran parte de las reservas existentes de algunos materiales (Valero et al., 2018). Además, varios autores plantean que futuros desarrollos de las tecnologías verdes como molinos eólicos, placas solares o vehículos eléctricos provocarán una escasez mayor de muchos materiales (Capellán-Pérez et al., 2019b; de Koning et al., 2018; Tokimatsu et al., 2017).

Además de los mencionados límites en los recursos que utiliza el ser humano, Rocksström et al., introdujeron en el año 2009 el concepto de "límites planetarios" (Rockström et al., 2009). Un límite planetario se refiere al punto específico relacionado con un proceso ambiental a escala mundial más allá del cual la humanidad no debería ir si se quieren evitar consecuencias desastrosas. Tras una revisión detallada se han establecido nueve límites planetarios que son los mostrados en la Figura 1.1 elaborada por Steffen et al., (Steffen et al., 2015). Los nueve límites son: cambio climático, nuevas entidades generadas por el ser humano, agotamiento del ozono estratosférico, carga de aerosoles atmosféricos, acidificación de los océanos, ciclos biogeoquímicos (fósforo y nitrógeno), uso del agua dulce, cambios en el uso de la tierra e integridad en la biosfera. El límite planetario se encuentra en el círculo grueso más interior de la Figura 1.1, justo donde se acaba la zona segura (verde) y empieza la zona de incertidumbre (amarillo). El circulo grueso exterior corresponde con el umbral crítico a partir del cual una pequeña perturbación puede alterar el estado o desarrollo de un sistema (tipping point). 


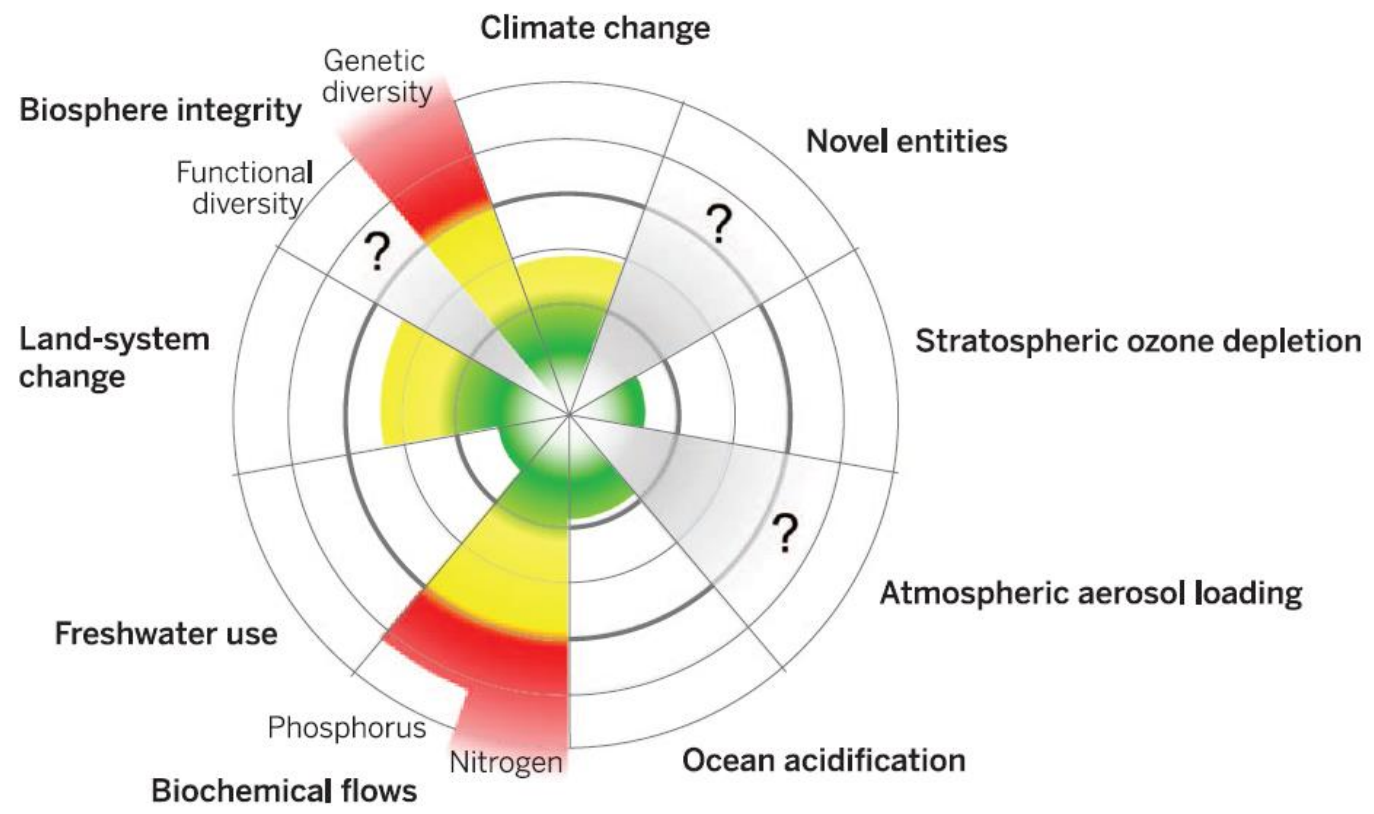

Beyond zone of uncertainty (high risk)

In zone of uncertainty (increasing risk)

Below boundary (safe)

Boundary not yet quantified

Figura 1.1: Estado actual de siete de los nueve límites planetarios.

Notas: La zona verde es la zona segura (por debajo del límite planetario), la zona amarilla representa la zona de incertidumbre en la que el riesgo aumenta y la zona roja es la zona de alto riesgo. El más interior de los círculos gruesos corresponde al límite planetario mientras que el círculo grueso exterior corresponde al umbral crítico. Las interrogaciones significan que el conocimiento existente sobre ese proceso ambiental es por ahora escaso.

Fuente: (Steffen et al., 2015).

Como puede verse en la Figura 1.1, detallada en el trabajo de Steffen et al. (Steffen et al., 2015), dos de los límites planetarios (integridad en la biosfera y ciclos biogeoquímicos) ya han superado el umbral crítico, y otros dos límites (cambio climático y cambios en el uso de la tierra) se encuentran en la zona de incertidumbre y son claves para evitar que el sistema cambie de forma irreversible. El conocimiento existente sobre la carga de aerosoles atmosféricos y las nuevas entidades que el hombre ha introducido o modificado en la naturaleza es muy escaso. Una de las principales limitaciones de la figura de Steffen es que no muestra las relaciones dinámicas que existen entre los distintos límites planetarios, dando a entender que son problemas diferenciados cuando en realidad están completamente interrelacionados.

Más recientemente, en el año 2017, Raworth incorporó un "fundamento social" a la visión "tecnoecológica" de los límites planetarios, donde se muestra lo que es seguro y justo para la humanidad añadiendo a la figura anterior un circulo interior como puede verse en la Figura 1.2 (Raworth, 2017). El fundamento social implica, entre otros aspectos, un acceso generalizado del ser humano al agua, los alimentos, la energía, la salud, la educación, la vivienda y la igualdad de género. Este comportamiento social, como se ha mencionado anteriormente, es uno de los aspectos claves para la sostenibilidad que, en una gran parte de las interpretaciones, implica una distribución equitativa de los recursos y de las oportunidades. 


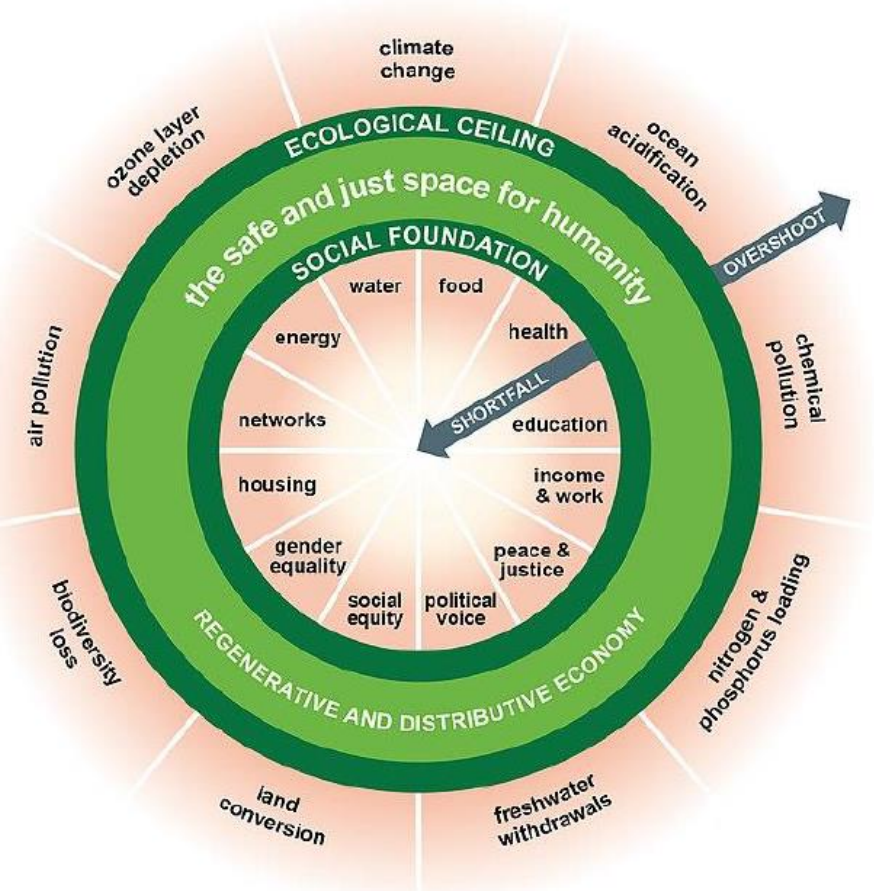

Figura 1.2: "Doughnut Economics".

Notas: Adaptación de la figura de los límites planetarios a los que se ha incorporado la parte social/económica para analizar lo que es seguro y justo para la humanidad.

Fuente: (Raworth, 2017).

La sostenibilidad implica también una equilibrada interrelación de la economía y la sociedad con el medio ambiente. Para el análisis de ambas ciencias se han utilizado generalmente distintos tipos de modelos. Los modelos son representaciones limitadas y simplificadas del mundo real que difieren de la realidad en muchísimos aspectos. Su objetivo es ayudar a describir, explicar o comprender mejor un problema de la realidad cuando no se puede trabajar directamente con él. Combinar las ciencias sociales con las medioambientales a través de los modelos es totalmente necesario para poder analizar la sostenibilidad del planeta. Para resolver esta difícil integración de ambas familias han surgido los modelos de energía-economía-medio ambiente o también llamados modelos de evaluación integrada (IAM). Un IAM es una herramienta de simulación numérica diseñada para ayudar a comprender las relaciones entre un gran número de variables de muy distintas áreas: tecnológicas, económicas, ambientales, sociales, etc. Existe una diversidad enorme entre los IAM. Una cuestión clave en el desarrollo de estos modelos son las realimentaciones entre los distintos subsistemas del modelo, especialmente las que relacionan las variables medioambientales con cuestiones económicas y sociales.

Los IAM son cada vez más utilizados para evaluar y analizar los efectos generados por la actividad humana sobre el planeta y para ayudar en la toma de decisiones políticas que permitan mantener la sostenibilidad en nuestra civilización (IPCC, 2014).

\subsection{Objetivos}

En el contexto descrito en la sección anterior, y con el objetivo de cubrir algunas de las limitaciones que los IAM actuales tienen en su intento de modelar el sistema energía-economíamedio ambiente, el grupo de Energía, Economía y Dinámica de Sistemas de la Universidad de 
Valladolid (GEEDS) utiliza la dinámica de sistemas como herramienta para analizar las complejas relaciones entre las variables que influyen en la sostenibilidad. A partir de trabajos sobre los límites de las energías fósiles y las energías renovables (de Castro et al., 2014, 2013, 2011, 2009; Mediavilla et al., 2013), GEEDS desarrolló en el año 2014 el modelo de simulación global "World Limits Model" WoLiM (Capellán-Pérez et al., 2017c, 2014). A partir del modelo WoLiM, y con el apoyo de los proyectos europeos MEDEAS ${ }^{1}$ y LOCOMOTION ${ }^{2}$, se ha desarrollado esta tesis doctoral con los siguientes objetivos:

Objetivo 1: Analizar la bibliografía existente sobre modelos de energía-economía-medio ambiente y especialmente aquellos que utilicen la dinámica de sistemas.

En la actualidad existen muchos modelos de energía-economía-medio ambiente (o IAM) con enfoques muy diferentes. Será necesario revisar la literatura existente sobre los IAM centrándose en los más utilizados por las principales instituciones y los modelos desarrollados en dinámica de sistemas. Esta revisión tendrá que ayudar a responder a las siguientes preguntas:

- ¿Por qué existen IAM tan diferentes, tanto en el enfoque utilizado como en los resultados que se obtienen?

- ¿Cuáles son los principales IAM utilizados por las instituciones y que les diferencia del resto?

- ¿Es la dinámica de sistemas una metodología apropiada para modelar los IAM? ¿Es una metodología muy utilizada dentro del conjunto de los IAM?

- ¿Cuáles son las limitaciones existentes en los principales IAM de la literatura?

- ¿Es necesario el desarrollo de un nuevo IAM a pesar de que existen actualmente muchos IAM diferentes o es mejor intentar mejorar los modelos ya existentes?

Objetivo 2: Construir y validar un modelo de energía-economía-medio ambiente utilizando la información histórica disponible correspondiente a cuestiones energéticas, económicas y medioambientales, así como los escenarios futuros que proponen los organismos internacionales.

El principal objetivo de esta tesis es construir y validar un modelo de energía-economía-medio ambiente que cubra las limitaciones encontradas en la revisión de la literatura sobre los IAM. Este modelo deberá basarse en los trabajos anteriores del GEEDS sobre los límites de las energías fósiles y las energías renovables. Será un modelo global, a nivel mundial, pero el marco de modelización tiene que ser adaptable a otros niveles de agregación regional, como la Unión Europea. El modelo se desarrollará conjuntamente con otros miembros del grupo de investigación GEEDS. Un nuevo modelo incorporado a la literatura tiene que dar respuesta a las siguientes preguntas:

- ¿Cuáles son las características incorporadas al modelo respecto a las existentes en otros IAM y cuáles son las ventajas de incorporarlas?

- ¿Qué potenciales aplicaciones tiene el modelo para analizar el sistema economía-energíamedio ambiente?

- ¿Cuáles son las principales limitaciones del modelo en las que se centre el trabajo futuro?

\footnotetext{
1 Proyecto MEDEAS: "Guiding European Policy toward a low-carbon economy. Modelling Energy system Development under Environmental And Socioeconomic constraints". (2016-2020). H2020-LCE-2015-2 (691287)

2 Proyecto LOCOMOTION: "Low-carbon society: an enhanced modelling tool for the transition to sustainability". (0106-2019 to 21-05-2023). H2020-LC-CLA-2018-2. Project Number 821105.
} 
- ¿Para qué nivel de agregación regional está diseñado? ¿Cómo se puede adaptar para utilizar el modelo en otros niveles de agregación?

- ¿Existe un informe en el que se expliquen las cuestiones técnicas del modelo (variables, ecuaciones, realimentación, etc.)? ¿Cómo se ha validado el modelo? ¿Está documentado su proceso de validación?

\section{Objetivo 3: Modelar la demanda energética, tanto de los distintos sectores económicos como de los hogares, considerando diferentes tipos de energía.}

Dentro del proceso de desarrollo de los IAM, estimar la demanda de energía es clave para el análisis de la transición energética y la elaboración de políticas alternativas eficaces. A su vez, las intensidades energéticas son un indicador muy relevante en la literatura de la eficiencia energética, ya que tienen una definición clara e intuitiva y un cálculo relativamente sencillo en los modelos. Por tanto, dentro de la construcción del IAM, modelar la demanda de energía a través de las intensidades energéticas será un objetivo importante de esta tesis. Este modelado tendrá que responder a las siguientes preguntas:

- ¿Cuáles son las principales variables que afectan a la demanda de energía y a las intensidades energéticas?

- ¿Cuáles son las potencialidades de la metodología de modelado elaborada? ¿Cómo evolucionarán bajo distintas condiciones las intensidades energéticas y la demanda de energía en el futuro?

- ¿Cuál es la incertidumbre del método planteado? ¿Cuál ha sido el proceso de validación? ¿Cómo se han calibrado los principales parámetros del modelo?

- ¿Es la metodología planteada adaptable a diferentes regiones, sectores o tipos de energía?

\section{Objetivo 4: Analizar distintas estrategias para descarbonizar el sector transporte, contemplando tanto opciones de electrificación y sustitución de vehículos como cambios en los modos de comportamiento de los ciudadanos.}

En la transición para alcanzar una sociedad más sostenible, el sector transporte es habitualmente identificado como uno de los sectores más difíciles de descarbonizar. Esto es debido, entre otros aspectos, al continuo crecimiento de la demanda mundial de movilidad y a las limitaciones técnicas para sustituir los combustibles derivados del petróleo. Estudiar distintas estrategias para descarbonizar el sector transporte utilizando el modelo desarrollado será otro objetivo importante de la tesis. Este análisis servirá de ejemplo de aplicación del modelo desarrollado y tendrá que responder las siguientes cuestiones:

- ¿Es posible descarbonizar el transporte siguiendo las tendencias actuales de sustitución de vehículos dependientes del petróleo por vehículos eléctricos? ¿Estamos cumpliendo los objetivos marcados por las principales instituciones?

- ¿Qué tipos de vehículos son los más adecuados para conseguir reducir las emisiones de $\mathrm{CO}_{2}$ del sector transporte?

- ¿Existen alternativas tecnológicas sostenibles para el transporte de mercancías?

- ¿Qué políticas son las más apropiadas para realizar la transición energética en el sector transporte? ¿Son compatibles estas políticas con nuestro actual modo de vida? 


\subsection{Metodología}

Como punto de partida, se revisarán los estudios realizados por el grupo de investigación GEEDS relacionados con los límites biofísicos de la energía y se analizará el modelo WoLiM en el que se han introducido dichos límites y se han relacionado con aspectos económicos y sociales. También se revisarán estudios similares encontrados en la literatura relacionados con los límites biofísicos.

Con el objetivo de adquirir el mayor conocimiento posible sobre los modelos de energíaeconomía-medio ambiente, se realizará una revisión de la literatura existente de estos modelos. Se pondrá especial atención en aquellos que utilicen la metodología de la dinámica de sistemas para el modelado. En el proceso de revisión de los IAM se analizarán las principales características de los modelos y cómo son implementadas matemáticamente en ellos. Se clasificarán los modelos y se buscarán sus principales cualidades y limitaciones. Se comparará el modelo WoLiM con los modelos existentes en la literatura y se planteará cómo mejorar el modelo y a la vez intentar cubrir las principales limitaciones encontradas durante la revisión de la literatura de los IAM.

Al mismo tiempo, se hará una revisión exhaustiva de los planes, programas y proyectos, tanto energéticos como medioambientales, definidos por las distintas instituciones y que están en proceso de implementación o que serán implementados en el futuro. Además, se recopilarán datos históricos de al menos los últimos 15 años sobre variables energéticas (oferta, demanda, transformación, etc.), económicas (demanda de los hogares, inversión, porcentaje de trabajo, etc.) y medioambientales (emisiones, uso de la tierra, recursos minerales, etc.)

Con toda la información recopilada, y basándose en el modelo WoLiM, se procederá al desarrollo de un modelo de evaluación integrada a nivel mundial mediante dinámica de sistemas, de tal manera que se puedan comprender y describir correctamente las relaciones dinámicas que existen entre las variables energéticas, económicas y medioambientales. La aplicación de la dinámica de sistemas permitirá incorporar retroalimentaciones de las que otras metodologías o modelos energéticos carecen. Durante el proceso de desarrollo se incluirán las técnicas de validación más relevantes en la literatura en el ámbito de la dinámica de sistemas y de los IAM. Se documentará con el mayor detalle posible tanto el proceso de modelado como el de validación. El desarrollo del modelo se realizará conjuntamente con otros miembros del grupo de investigación GEEDS.

El marco de modelización utilizado para desarrollar el modelo mundial se adaptará para poder utilizarse en otros niveles de agregación regional, principalmente a nivel europeo. Para ello se introducirán en el modelo nuevas características que no se modelan a nivel mundial, como el comercio entre regiones, y también se estudiará cómo relacionar los distintos modelos para que los resultados sean coherentes entre sí.

Por último, se utilizará el modelo para analizar las posibles políticas a aplicar para una transición sostenible. Estas políticas podrán ser energéticas, ambientales, económicas y tecnológicas, pero esta tesis se centrará en aquellas políticas que afectan al sector transporte, uno de los más difíciles de descarbonizar para alcanzar una transición energética sostenible. Se estudiarán diferentes opciones de electrificación y sustitución de vehículos y también cambios en los modos de comportamiento de los ciudadanos.

\subsection{Organización de la tesis}

La tesis está dividida en cuatro partes: 
- La parte I está formada por la introducción (capítulo 1), en la que se presenta el contexto, objetivos y metodología de esta tesis, y por una revisión de la literatura de los principales modelos de energía-economía-medio ambiente (capítulo 2).

- La parte II es la parte principal de esta tesis, en ella se muestra el desarrollo de un nuevo marco de modelización para analizar el sistema energía-economía-medio ambiente. El marco de modelización se llama MEDEAS debido al proyecto europeo homónimo dentro del cual se ha desarrollado. Esta parte está compuesta por 3 capítulos, los dos primeros (capítulos 3 y 4) explican cuestiones del modelado de MEDEAS y el capítulo 5 muestra el proceso de validación realizado a MEDEAS.

- La parte III muestra un ejemplo de aplicación de MEDEAS en el que se estudian distintas estrategias de descarbonización en el sector transporte (capítulo 6).

- La parte IV está formada por el capítulo 7 que muestra las conclusiones de esta tesis, remarcando los principales resultados y señalando las futuras líneas de desarrollo de MEDEAS.

Los capítulos 2, 3, 4 y 6 de esta tesis se corresponden con artículos que han sido publicados en revistas científicas de impacto y que se muestran en esta tesis en el idioma en el que han sido publicados, como es el caso de los capítulos 3, 4 y 6 que están escritos en inglés.

El capítulo 2 titulado "Revisión de modelos de energía-economía-medio ambiente (IAM)" es una versión más detallada del artículo "Modelos de Evaluación Integrada (IAM) aplicados al cambio climático y la transición energética" en el que se muestran las principales características y limitaciones de los IAM revisados en la literatura. El contenido mostrado en este capítulo ha sido publicado en la revista española DYNA como: De Blas, I., Miguel, L.J., De Castro, C., 2021. Integrated assessment models (IAMs) applied to climate change and energy transition. DYNA Ingeniería e Industria 96, 316-321. https://doi.org/10.6036/9922.

El capítulo 3 titulado "MEDEAS: un nuevo marco de modelización que integra las limitaciones biofísicas y socioeconómicas mundiales" describe el marco de modelización de los modelos MEDEAS que permite analizar las interacciones energéticas, económicas y medioambientales durante la transición energética. Además, este capítulo muestra las potencialidades que aporta el nuevo marco de modelización mediante la simulación de cuatro variantes de las tendencias actuales en el modelo MEDEAS-World (MEDEAS-W). Este capítulo ha sido publicado como: Capellán-Pérez, I., de Blas, I., Nieto, J., Castro, C. de, Miguel, L.J., Carpintero, Ó., Mediavilla, M., Lobejón, L.F., Ferreras-Alonso, N., Rodrigo, P., Frechoso, F., Álvarez-Antelo, D., 2020. MEDEAS: a new modeling framework integrating global biophysical and socioeconomic constraints. Energy Environ. Sci. 13, 986-1017. https://doi.org/10.1039/C9EE02627D. El objetivo de este artículo es describir de una manera detallada el nuevo marco de modelización desarrollado a lo largo de los cuatro años de proyecto MEDEAS, por lo que aparecen como autores todos los investigadores que han participado de alguna manera en su desarrollo. Es necesario remarcar que el desarrollo de MEDEAS ha sido un trabajo conjunto del grupo de investigación GEEDS y que las principales tareas que ha realizado el autor de esta tesis doctoral han sido el desarrollo de la parte energética, especialmente el modelado de la demanda de energía y del sector transporte, y la integración técnica de todos los submódulos dentro del modelo general, incluido la realización de las pruebas de validación.

El capítulo 4 titulado "Modelización de la demanda energética sectorial a través de las intensidades energéticas en los modelos de evaluación integrada MEDEAS" describe un novedoso método de estimación de la demanda energética en el nuevo marco de modelización MEDEAS basado en la evolución de las intensidades energéticas sectoriales. En el capítulo se 
muestra la potencialidad del método desarrollado mediante el análisis de distintos casos de estudio en MEDEAS-W. Este capítulo ha sido publicado como: de Blas, I., Miguel, L.J., Capellán-Pérez, I., 2019. Modelling of sectoral energy demand through energy intensities in MEDEAS integrated

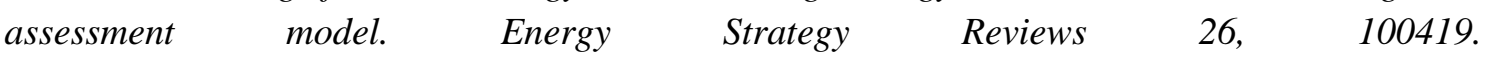
https://doi.org/10.1016/j.esr.2019.100419.

El capítulo 6 titulado: "Los límites de la descarbonización del transporte en el actual paradigma de crecimiento" estudia cuatro estrategias de descarbonización del sector transporte a nivel mundial para el año 2050, utilizando el modelo de evaluación integrada MEDEAS-W. En el capítulo se analizan los efectos de aplicar dichas estrategias a nivel económico, energético, climático y en cuanto al consumo de materiales. Este capítulo ha sido publicado como: de Blas, I., Mediavilla, M., Capellán-Pérez, I., Duce, C., 2020. The limits of transport decarbonization under the current growth paradigm. Energy Strategy Reviews 32, 100543. https://doi.org/10.1016/j.esr.2020.100543.

La Figura 1.3 muestra la estructura de esta tesis señalando los diferentes capítulos y los principales objetivos.

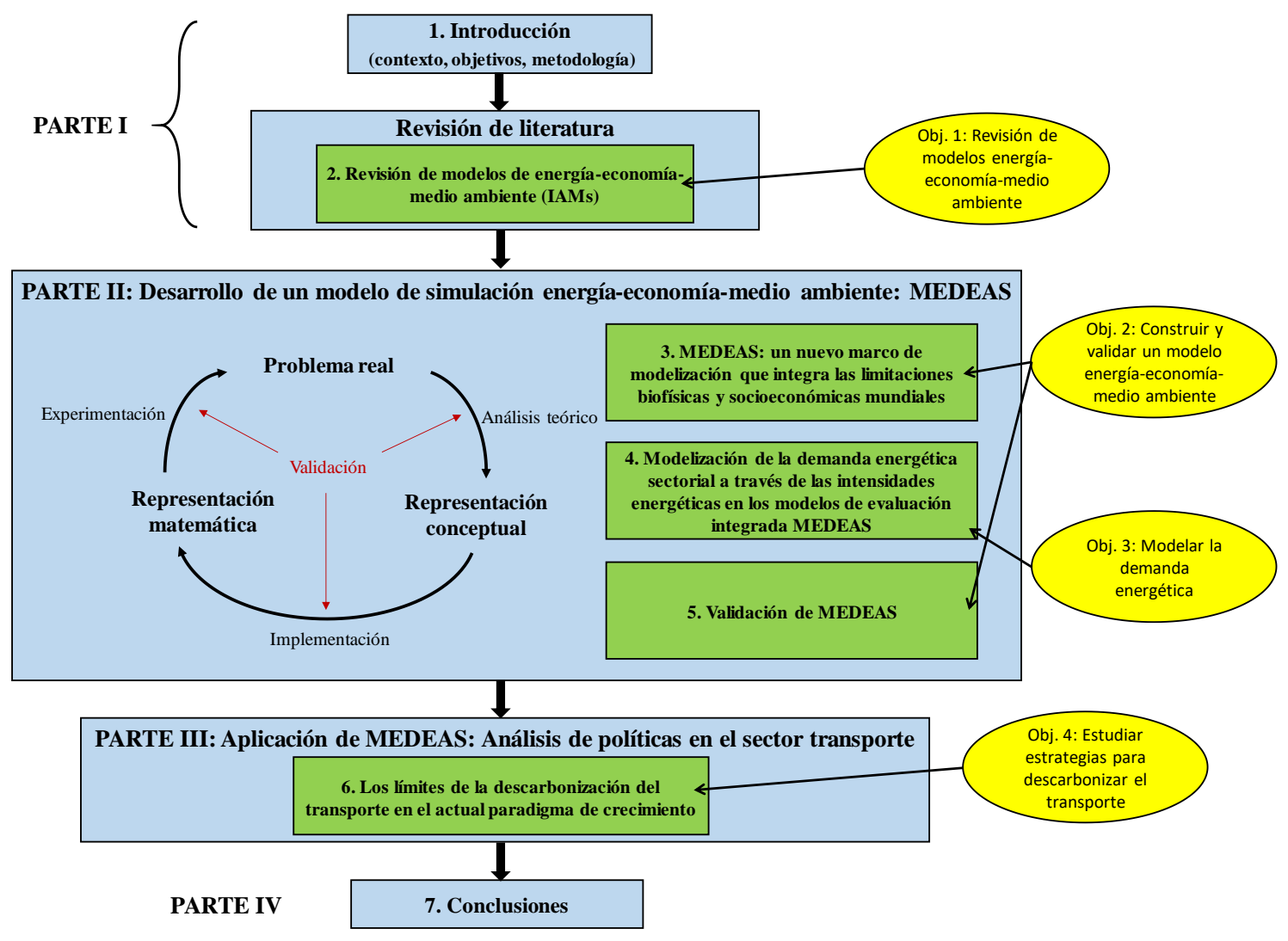

Figura 1.3: Estructura de la tesis.

Notas: Se muestran las cuatro partes, los siete capítulos y los cuatro objetivos principales de esta tesis. 


\section{Revisión de modelos de energía-economía-medio ambiente (IAM)}

$\underline{\text { Referencia: }}$

De Blas, I., Miguel, L.J., De Castro, C., 2021. Integrated assessment models (IAMs) applied to climate change and energy transition. DYNA Ingeniería e Industria 96, 316-321. https://doi.org/10.6036/9922.

\section{Resumen:}

El cambio climático que actualmente se está produciendo es debido al incremento de la concentración de gases de efecto invernadero (GEI) en la atmósfera como consecuencia de la actividad humana. El gran número de factores y variables que, de manera directa o indirecta, afectan a las emisiones de GEI, así como las múltiples y complejas relaciones entre ellas, dificulta la toma de decisiones sobre las mejores medidas que deben adoptarse para frenar o paliar el cambio climático y el análisis de las consecuencias que cada decisión arrastra. Ello ha motivado el desarrollo de complejos modelos de simulación denominados Modelos de Evaluación Integrada (IAMs) o de Energía-Economía-Medio Ambiente (modelos E3), centrados especialmente en el cambio climático. El desarrollo y uso de estos modelos para orientar las decisiones políticas en materia de cambio climático ha crecido muy notablemente en los últimos años, como se pone de manifiesto en los informes del Grupo Intergubernamental de Expertos sobre el Cambio Climático (IPCC). Este trabajo es una revisión panorámica de los principales IAMs existentes y analiza sus principales características. El trabajo se centra especialmente en el análisis de las limitaciones de los IAMs actuales, que deberían marcar los desarrollos futuros de estas herramientas. 


\section{PARTE II: DESARROLLO DE UN MODELO DE ENERGÍA- ECONOMÍA-MEDIO AMBIENTE: MEDEAS $^{3}$}

\section{MEDEAS: un nuevo marco de modelización que integra las limitaciones biofísicas y socioeconómicas mundiales}

\section{Referencia:}

Capellán-Pérez, I., Blas, I. de, Nieto, J., Castro, C. de, Miguel, L.J., Carpintero, Ó., Mediavilla, M., Lobejón, L.F., Ferreras-Alonso, N., Rodrigo, P., Frechoso, F., Álvarez-Antelo, D., 2020. MEDEAS: a new modeling framework integrating global biophysical and socioeconomic constraints. Energy Environ. Sci. 13, 986-1017. https://doi.org/10.1039/C9EE02627D.

\section{Resumen:}

A diversity of integrated assessment models (IAMs) coexists due to the different approaches developed to deal with the complex interactions, high uncertainties and knowledge gaps within the environment and human societies. This paper describes the open-source MEDEAS modeling framework, which has been developed with the aim of informing decision-making to achieve the transition to sustainable energy systems with a focus on biophysical, economic, social and technological restrictions and tackling some of the limitations identified in the current IAMs. MEDEAS models include the following relevant characteristics: representation of biophysical constraints to energy availability; modeling of the mineral and energy investments for the energy transition, allowing a dynamic assessment of the potential mineral scarcities and computation of the net energy available to society; consistent representation of climate change damages with climate assessments by natural scientists; integration of detailed sectoral economic structure (input-output analysis) within a system dynamics approach; energy shifts driven by physical scarcity; and a rich set of socioeconomic and environmental impact indicators. The potentialities and novel insights that this framework brings are illustrated by the simulation of four variants of current trends with the MEDEAS-world model: the consideration of alternative plausible assumptions and methods, combined with the feedback-rich structure of the model, reveal dynamics and implications absent in classical models. Our results suggest that the continuation of current trends will drive significant biophysical scarcities and impacts which will most likely derive in regionalization (priority to security concerns and trade barriers), conflict, and ultimately, a severe global crisis which may lead to the collapse of our modern civilization. Despite depicting a much more worrying future than conventional projections of current trends, we however believe it is a more realistic counterfactual scenario that will allow the design of improved alternative sustainable pathways in future work.

\footnotetext{
${ }^{3}$ The development of MEDEAS has been a joint work of the GEEDS research group and the main tasks performed by the author of this doctoral thesis have been the development of the energy modules, especially the modelling of the energy demand and the transportation sector, and the technical integration of all the submodules in the general model, including the validation tests.
} 


\section{Modelización de la demanda energética sectorial a través de las intensidades energéticas en los modelos de evaluación integrada MEDEAS}

Referencia:

de Blas, I., Miguel, L.J., Capellán-Pérez, I., 2019. Modelling of sectoral energy demand through energy intensities in MEDEAS integrated assessment model. Energy Strategy Reviews 26, 100419. https://doi.org/10.1016/j.esr.2019.100419.

\section{Resumen:}

The estimation of future energy demand is a key factor for the development of effective alternative policies towards a low carbon economy. This paper describes a novel method to estimate the energy demand in the new integrated assessment framework MEDEAS based on the projection of sectoral final energy intensities. The dynamic of each of the sectoral final energy intensity is broken down into (1) improvement in energy efficiency and (2) substitution of the final energy. The speed of changes in these factors depend on physical supply-demand unbalances in the market, climate mitigation and other energy saving policies and the perception of scarcity of the different economic agents. The simulated case studies in MEDEAS-World under the narrative of the Business-as-usual (BAU) scenario have allowed validating the model's robustness and showing the potentiality of its application. 


\section{Validación de MEDEAS}

\subsection{Introducción a la validación de modelos}

\subsubsection{Validación de modelos de simulación}

Validar un modelo es demostrar que dentro de su ámbito de aplicabilidad posee un rango satisfactorio de precisión compatible con la aplicación prevista del modelo (Sargent, 2013). Esta definición utilizada por Sargent es una de las más utilizadas para la validación de modelos de simulación. Sin embargo, el proceso de validación siempre ha estado muy abierto a debate debido, entre otras cosas, a la subjetividad de lo que puede ser "un rango satisfactorio de precisión" o al significado de la palabra validación (Oreskes, 1998; Oreskes et al., 1994; Sterman, 2000). La forma y los métodos de validar los modelos de simulación también han sido con frecuencia motivo de discusión entre los desarrolladores de modelos (Balci, 1994; Barlas, 1996). De lo que apenas hay dudas en la comunidad científica es que demostrar la credibilidad del modelo es crucial para que genere confianza a sus usuarios.

Como se ha explicado en el capítulo segundo de esta tesis, los modelos pueden clasificarse de muchas maneras. En lo relativo a la validación, una diferencia muy importante entre modelos es si son "causales-descriptivos" (cajas blancas) o modelos puramente "correlativos" (cajas negras) (Barlas, 1996). En los modelos considerados "cajas negras", al no haber relaciones de causalidad en el modelo, la única manera de validarlos es comprobar si sus resultados coinciden con los resultados "reales" dentro de un nivel definido de precisión. Por otra parte, en los modelos "cajas blancas" tener gran precisión en los resultados no es suficiente para validar el modelo, lo que es realmente importante es validar la estructura interna del modelo (Barlas, 1996). Estos modelos no solo reproducen/predicen el comportamiento de un sistema, sino que también explican cómo se genera el comportamiento y permiten sugerir cambios en dicho comportamiento. La validación de los modelos conocidos como "cajas blancas" es mucho más compleja que la de los modelos "cajas negras". Los problemas para la validación de los modelos "cajas blancas" son tanto técnicos como filosóficos. Técnicamente es difícil porque no existe un consenso sobre qué pruebas formales se pueden usar para decidir si el modelo está suficientemente cerca de la estructura "real", siendo unas pruebas adecuadas para unos modelos, pero no para otros.

Además, la validación de los modelos está directamente relacionada con la cuestión filosófica no resuelta de verificar la verdad de una declaración científica. Esto quiere decir que nuestra validación del modelo depende de nuestra metodología de obtención y confirmación del conocimiento (Barlas, 1996). En su artículo, Barlas señala dos principales corrientes de pensamiento: la escuela reduccionista/positivista y la escuela relativista/holística. Para la escuela reduccionista/positivista, el modelo puede ser "correcto" o "incorrecto", y una vez el modelo se confronta con la realidad su validez o falsedad quedan automáticamente demostradas. En esta corriente la validación se entiende como una cuestión de precisión más que de utilidad (Barlas and Carpenter, 1990). Por el contrario, para la escuela relativista/holística un modelo válido es una de las posibles maneras de describir una situación real. Ningún modelo puede tener absoluta objetividad, los modelos no son verdaderos o falsos, para validar los modelos se tiene en cuenta su utilidad. Según esta escuela, la validación de la estructura interna de un modelo no puede ser totalmente objetiva, formal y cuantitativa. Una revisión más detallada de los principales fundamentos epistemológicos sobre la validez de los modelos se puede encontrar en el trabajo de Schwaninger y Groesser (Schwaninger and Groesser, 2020).

Muy clara tiene esta cuestión Sterman, quien afirma que la verificación y validación de los modelos es imposible (Sterman, 2000). Como explica en su libro, todos los modelos están en 
cierto sentido equivocados, ya que son representaciones limitadas y simplificadas del mundo real. Los modelos difieren de la realidad en muchísimos aspectos. La imposibilidad de la validación en el sentido de establecer la verdad también ha sido reflejada por otros autores como Oreskes, Shrader-Frechette y Belitz, quienes escribían que "los modelos son representaciones útiles para guiar el estudio posterior pero no susceptibles de ser probados" (Oreskes et al., 1994). En el mismo sentido Forrester decía (Forrester, 1961):

"Any "objective" model-validation procedure rests eventually at some lower level on a judgment or faith that either the procedure or its goals are acceptable without objective proof."

Además, es esencial un proceso de comunicación entre los modeladores y los usuarios de los modelos para estudiar su utilidad. Por tanto, será necesaria una integración entre puntos de vista subjetivos y la creación de un consenso, por lo que la validación de modelos es también intrínsecamente social (Sterman, 2000).

Una vez abandonado el dualismo de modelos correctos e incorrectos, y reconociendo que hay un comportamiento social y totalmente subjetivo en la validación de los modelos, es necesario marcar una serie de pautas y técnicas que, de manera científica, ayuden a evaluar los modelos y aumentar la confianza de los modeladores y usuarios. Algunas de estas pautas se describen en los siguientes párrafos.

La primera cuestión en el desarrollo de un modelo, que resultará clave para su validación, es definir correctamente la finalidad del modelo. En el momento en que se une la validez de un modelo con su utilidad es imposible definir la validez separada de cuál es su finalidad. Así que es totalmente necesario especificar, con la mayor claridad posible, la finalidad del modelo, incluyendo sus usos previstos y el grupo de usuarios que va a tener.

Las pruebas de validación tienen que estar integradas dentro del proceso de modelado, hay que entender la validación como un proceso iterativo de pruebas que se tienen que hacer durante todo el desarrollo del modelo. Al evaluar continuamente los distintos pasos realizados en el desarrollo se descubren errores importantes con antelación, se evita tener que repetir con posterioridad partes del modelo y se generan conocimientos para los pasos posteriores. En su versión simplificada del proceso de desarrollo de los modelos de simulación, y como se puede observar en la Figura 5.1, Sargent separa en tres los tipos de validaciones durante el proceso de desarrollo: validación del modelo conceptual, validación de la programación del modelo y validación de la aplicabilidad de modelo (Sargent, 2013). Estas validaciones se deben realizar cada vez que se hace un cambio en el modelo. El proceso de validación se tiene que repetir tantas veces como sea necesario hasta conseguir demostrar la validez del modelo. 


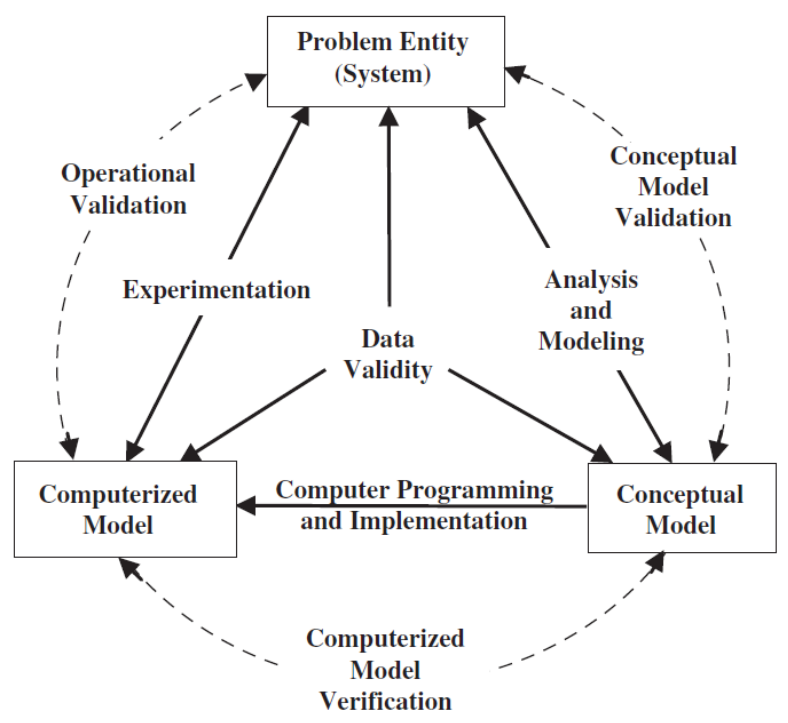

Figura 5.1: Versión simplificada del proceso de desarrollo de un modelo con sus fases de validación.

Fuente: (Sargent, 2013).

A los modelos se les pueden realizar muchas pruebas en cualquiera de las etapas de su desarrollo, pero desafortunadamente no existen unas pruebas específicas que permitan determinar con claridad la validez de un modelo. Algunas de las pruebas son válidas para unos modelos, pero no para otros. Por lo general, cuantas más pruebas se realicen al modelo mayor confianza se podrá tener en que el modelo refleje el comportamiento correctamente (Featherston and Doolan, 2012).

Una de las cuestiones más importante para los desarrolladores es la documentación del modelo (Sterman, 2000). Por lo general, documentar los modelos es una tarea frecuentemente descuidada, pero la documentación es necesaria para asegurar que los resultados puedan ser comprendidos, replicados, criticados y extendidos por otros. Sin la documentación adecuada, el trabajo no es ni científico ni útil. Además, es necesario documentar la utilidad del modelo describiendo el proceso de validación y publicando las conclusiones de las pruebas realizadas. Por consiguente, documentar tanto el modelo como su proceso de validación es totalmente necesario para demostrar la utilidad de un modelo.

\subsubsection{Validación en dinámica de sistemas}

Los modelos de dinámica de sistemas son herramientas muy útiles para el análisis de políticas debido, entre otras causas, a su capacidad de mostrar las relaciones dinámicas entre distintas partes del sistema. Sin embargo, a pesar de sus capacidades, la aceptación de estos modelos por la comunidad más amplia de modeladores y encargados de tomar decisiones es bastante limitada. Una de las principales razones es la resistencia de los modeladores en dinámica de sistemas a exponer sus modelos a procedimientos de validación formal (Qudrat-Ullah, 2012). Sus procedimientos subjetivos y calificativos han sido comúnmente criticados (Featherston and Doolan, 2012).

Como se decía en el apartado anterior, para definir la validez de un modelo es imposible separarla de su utilidad, así que para determinar si un modelo de dinámica de sistemas cumple con la finalidad marcada es necesario un proceso no técnico, informal y cualitativo. Además, en los 
modelos de simulación, y por consiguiente en los modelos de dinámica de sistemas, es necesario validar la estructura interna del modelo para poder hacer una validación completa del mismo.

Esto no quiere decir que se rechace por completo el uso de mecanismos formales y cuantitativos en la validación de la dinámica de sistemas, sino todo lo contrario. Es necesario establecer un proceso de validación con métodos rigurosos y formales para validar conjuntamente con las valoraciones subjetivas e informales. Varios han sido los intentos en la literatura de establecer un conjunto de pruebas para validar los modelos de dinámica de sistemas como, por ejemplo, las pruebas para aumentar la confianza en los modelos elaboradas por Forrester y Senge (Forrester and Senge, 1980). Otros autores también han tratado de elaborar un proceso de validación de los modelos de dinámica de sistemas con distintos tipos de pruebas (Barlas, 1996; Schwaninger and Groesser, 2020; Sterman, 2000). Por ejemplo, Barlas estableció una secuencia lógica de pruebas formales para validar los modelos de dinámica de sistemas que se muestra en la Figura 5.2.

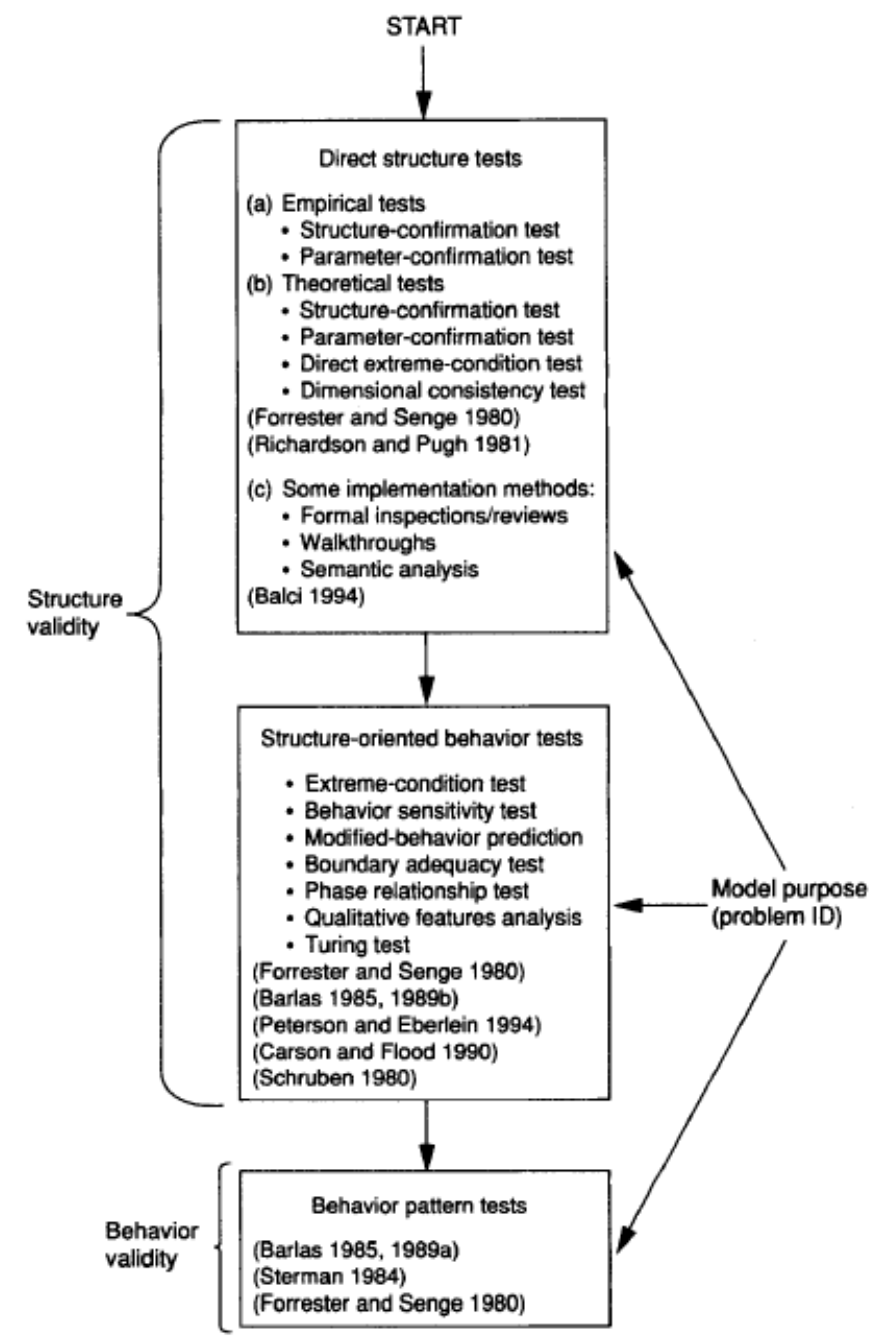

Direct structure tests

Figura 5.2: Secuencia lógica de las pruebas a realizar para la validación de un modelo de dinámica de sistemas.

Fuente: (Barlas, 1996).

Como se observa en la Figura 5.2, se pueden distinguir dos tipos de pruebas para analizar la estructura interna del modelo: las pruebas directas de estructura y las pruebas de 
comportamiento orientadas a la estructura. Las pruebas directas de estructura evalúan la validez de la estructura del modelo a través de una comparación directa con el conocimiento existente sobre la estructura real del sistema. Las pruebas de comportamiento orientadas a la estructura evalúan la validez de la estructura indirectamente aplicando pruebas en patrones de comportamiento generados por los modelos. Este segundo tipo de pruebas pueden implicar una simulación, pueden estar aplicadas a todo el modelo, así como a submodelos del mismo y ayudan al modelador a descubrir potenciales fallos estructurales (Barlas, 1996).

Una vez realizadas suficientes pruebas para validar la estructura del modelo con las dos categorías descritas anteriormente, el modelador tendrá la confianza necesaria para empezar a aplicar pruebas dedicadas a medir con cuanta precisión se reproducen en el modelo los patrones de comportamiento mostrados por el sistema real. Es importante remarcar que el objetivo es comparar los patrones de comportamiento (periodos, frecuencias, tendencias, amplitudes) más que la predicción de puntos exactos.

Aunque en la Figura 5.2 se establece una secuencia lógica de las pruebas, no hay que olvidar que la validación es un proceso iterativo de pruebas y que según estas se van realizando y se van encontrando posibles fallos habrá que volver hacia atrás a modificar cuestiones de la estructura del modelo. La Figura 5.3, elaborada también por Barlas, muestra ese proceso iterativo en la elaboración/validación de un modelo.

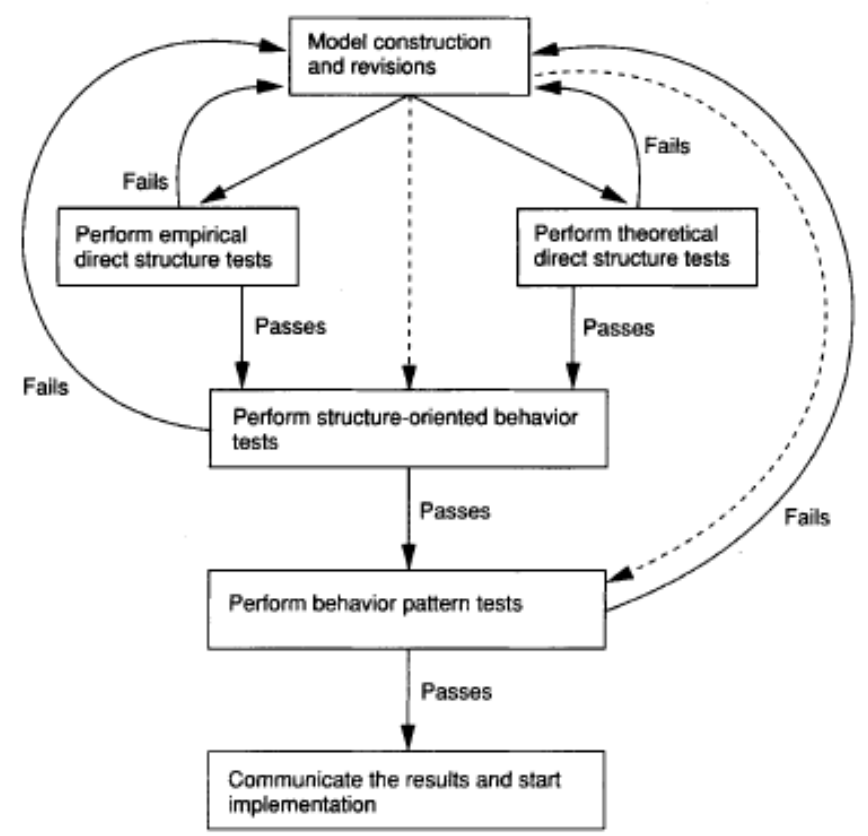

Figura 5.3: Secuencia lógica de desarrollo de un modelo con sus pruebas de validación.

Fuente: (Barlas, 1996).

Como se ve en la Figura 5.3, una vez realizadas las pruebas que evalúan los patrones de comportamiento, el siguiente paso es comunicar los resultados del proceso de validación. Para ello es necesario que, según se vayan realizando las pruebas al modelo y se vayan obteniendo sus resultados, se documente con detalle toda esa información.

En trabajos más actuales como los de Sterman (Sterman, 2000) y Schwaninger y Grosses (Schwaninger and Groesser, 2020), el proceso de validación de los modelos es muy similar al elaborado por Barlas. En los respectivos trabajos de los autores anteriores está perfectamente 
detallada la documentación donde se explica cómo realizar las distintas pruebas de validación. Es necesario remarcar que ninguna prueba es la más adecuada, una amplia gama de pruebas ayuda a la comprobar la robustez del modelo y comprender sus limitaciones (Sterman, 2000).

Por último, para este tipo de modelos, hay que intentar ampliar el proceso de validación al rango más amplio posible de personas fuera de los desarrolladores. Una buena decisión es hacer que los diferentes usuarios del modelo participen en el proceso de validación realizando ellos diferentes pruebas. Otra opción para aumentar la credibilidad del modelo es que sean personas totalmente ajenas al modelo las que realicen una verificación y validación independiente. Cuando la validación se ha llevado a cabo por personas independientes y estos concluyen que el modelo es válido, hay una probabilidad mucho mayor de que el modelo sea aceptado y se consideren los resultados del modelo como válidos (Sargent, 2013).

\subsubsection{Validación de los IAM}

Los modelos de evaluación integrada (IAM), como ya se ha explicado anteriormente, son modelos complejos diseñados para ayudar a comprender las relaciones entre un gran número de variables tecnológicas, económicas, ambientales y sociales. Los resultados obtenidos en los IAM se utilizan para tomar decisiones muy importantes sobre políticas climáticas y energéticas (IPCC, 2014). Es necesario entonces preguntarse hasta qué punto estos modelos tan complejos proporcionan respuestas fiables y qué márgenes de incertidumbre tienen (NCC, 2015; Schwanitz, 2013). Cómo validar los IAM ha generado una gran preocupación en la comunidad de desarrolladores y usuarios de los IAM y por ahora no se ha resuelto de manera definitiva, no existen normas ni protocolos claros para su validación (Alexandrov et al., 2011; Risbey et al., 1996).

Junto a las limitaciones comentadas en la sección ¡Error! No se encuentra el origen de la referencia. de esta tesis, no acompañar a los modelos de su proceso de validación ha generado distintas críticas a lo largo de la historia de los IAM. Algunas de las cuestiones más planteadas a los desarrolladores de los IAM son: ¿Cómo se sabe que los resultados de situaciones futuras que nos proporcionan los modelos son válidos? ¿Se ha probado a simular en años anteriores y ver si el modelo predice el presente? Más allá del enfoque bastante equivocado que algunas de estas críticas tienen sobre el objetivo de los IAM, que recordamos que no es predecir el futuro, sino proporcionar representaciones viables de lo que puede ocurrir en el futuro, es esencial establecer un protocolo para poder validar los modelos y generar confianza a sus posibles usuarios.

Con ese objetivo, el consorcio IAMC, creó en el año 2013 el "Grupo de trabajo en evaluación y diagnóstico de los IAM" (IAMC, 2020). Algunos de los objetivos de este grupo de trabajo son: identificar los métodos de evaluación y diagnóstico más utilizados por los desarrolladores de los IAM, generar un debate sobre la evaluación de los IAM incluyendo expertos de fuera del consorcio, recomendar unas pruebas comunes para la evaluación de los modelos y fomentar la documentación de los modelos. Algunas actividades ya realizadas por este grupo son la obtención de un documento de trabajo sobre como evaluar los IAM (Wilson et al., 2017), o la elaboración de la "wiki" online (https://www.iamcdocumentation.eu/index.php/IAMC_wiki). Las primeras conclusiones del grupo de trabajo del IAMC ratifican lo comentado en los apartados anteriores sobre validación de modelos.

Los IAM por sus características se tienen que acercar más a la idea de "cajas blancas" que a la de "cajas negras" (Schwanitz, 2013). Al no tener como objetivo obtener unos resultados concretos, sino ayudar a la toma de decisiones basado en representaciones de lo que puede ocurrir en un futuro, la validación de la estructura interna es igual de importante que la validación del 
comportamiento del modelo. La estructura interna de un IAM está formada por parámetros, variables, ecuaciones, principios de causalidad, etc., y la mayoría de estos elementos presenta incertidumbres que, por lo general, no han sido muy evaluadas en los IAM (Pastor et al., 2020). También hay que considerar, a la hora de analizar ecuaciones y relaciones, que las leyes y procesos fundamentales en la naturaleza se conocen casi siempre de manera incompleta.

En relación a la validación del comportamiento del modelo, hay que tener en cuenta que validar modelos complejos como los IAM tiene muchas limitaciones (Wilson et al., 2017). Wilson et al., en su artículo especifica alguna de ellas. En primer lugar, los resultados de la simulación pueden ser debidos a parametrizaciones afinadas y así mostrar solo "adecuación empírica forzada". En segundo lugar, existe más de un modelo que puede proporcionar los mismos resultados, lo que implica que no hay una solución única. Tercero, dos o más errores en los parámetros o variables del modelo pueden anularse entre sí. Todo esto conlleva a que una buena precisión en los resultados de un modelo no significa necesariamente que el modelo sea correcto. También lo contrario, que los resultados no coincidan no implica que el modelo esté mal, pues esto puede deberse, por ejemplo, a errores en las condiciones iniciales. Además, la evaluación de los IAM, como dice Wilson et al., se enfrenta a otros muchos problemas como, por ejemplo, que la validación solo puede realizarse haciendo una retrospectiva, es decir, utilizando valores históricos, y además, una precisa representación del periodo histórico no implica que en el futuro vaya a seguir ese comportamiento.

Por tanto, teniendo en cuenta la naturaleza de los IAM y las limitaciones existentes en la evaluación de los modelos, el grupo de trabajo del IAMC establece que los IAM tienen que cumplir cinco criterios teóricos (Wilson et al., 2017):

- $\quad$ Adecuación: el modelo tiene que estar orientado para su finalidad y diseño.

- Interpretabilidad: la forma en la que el modelo esta conceptualizado y representado tiene que ser clara para el análisis y comunicación de sus resultados.

- Verificabilidad: el código del modelo debe documentarse de manera clara y transparente para permitir un análisis independiente.

- Credibilidad: tanto los modeladores como los usuarios tienen que tener una gran confianza en el modelo como herramienta de análisis.

- Utilidad: los modelos deben fomentar la comprensión de las políticas y retos.

Para poder cumplir estos criterios, y teniendo en cuenta que la evaluación de un modelo es un proceso iterativo de múltiples pruebas, correcciones y mejoras, el grupo de trabajo del IAMC distingue ocho métodos de evaluación de los IAM con sus ventajas y limitaciones como puede verse en la Tabla 5.1. 
Tabla 5.1: Ventajas y limitaciones de ocho métodos de evaluación de los IAM.

Fuente: (Wilson et al., 2017).

\begin{tabular}{|c|c|c|}
\hline $\begin{array}{l}\text { Evaluation } \\
\text { method }\end{array}$ & Strengths & Limitations \\
\hline $\begin{array}{l}\text { historical } \\
\text { simulations }\end{array}$ & $\begin{array}{l}\text { - use of observations } \\
\text { - behavioral validity test }\end{array}$ & $\begin{array}{l}\text { - not as relevant for normative model } \\
\text { applications (e.g., cost-effective pathways) } \\
\text { - not predictive of future conditions } \\
\text { - difficulty of separating forcings from } \\
\text { system responses in dynamic baselines } \\
\text { - limited to specific causal mechanisms or } \\
\text { spatial scales } \\
\text { - limited implications for structural validity } \\
\text { (and issues with over-tuning) }\end{array}$ \\
\hline $\begin{array}{l}\text { near-term } \\
\text { observations }\end{array}$ & $\begin{array}{l}\text { - use of observations } \\
\text { - behavioral validity test }\end{array}$ & $\begin{array}{l}\text { - models not designed to capture short-term } \\
\text { variability } \\
\text { - (other limitations as for historical } \\
\text { simulations) }\end{array}$ \\
\hline $\begin{array}{l}\text { generalizable } \\
\text { historical } \\
\text { patterns }\end{array}$ & $\begin{array}{l}\text { - use of patterns in observations } \\
\text { - broad applicability (less sensitive to } \\
\text { specific historical conditions and data } \\
\text { constraints) } \\
\text { - contributes to behavioral validity }\end{array}$ & $\begin{array}{l}\text { - subjective comparisons, no standardized } \\
\text { tests } \\
\text { - difficulty of identifying reasons for } \\
\text { divergence and implications for structural } \\
\text { validity }\end{array}$ \\
\hline $\begin{array}{l}\text { hierarchy of } \\
\text { models \& simple } \\
\text { models }\end{array}$ & $\begin{array}{l}\text { - tests understanding of key system } \\
\text { processes } \\
\text { - links model behavior to structural } \\
\text { validity } \\
\text { - clearly interpretable results }\end{array}$ & $\begin{array}{l}\text { - limited applicability and policy-relevance } \\
\text { - difficulty in simplifying heterogeneous } \\
\text { causal mechanisms }\end{array}$ \\
\hline $\begin{array}{l}\text { model inter- } \\
\text { comparison } \\
\text { projects (MIPs) }\end{array}$ & $\begin{array}{l}\text { - identify results robust to (and sensitive } \\
\text { to) structural uncertainty } \\
\text { - insights on structural validity } \\
\text { - peer review, exchange of data and } \\
\text { methods, tacit leaming among } \\
\text { modelling teams }\end{array}$ & $\begin{array}{l}\text { - limited standardization of scenario } \\
\text { implementation across diverse models } \\
\text { - difficulty of attributing divergent results to } \\
\text { individual model differences } \\
\text { - risk of groupthink in shared modelling } \\
\text { strategies, removal of outliers }\end{array}$ \\
\hline $\begin{array}{l}\text { diagnostic } \\
\text { indicators }\end{array}$ & $\begin{array}{l}\text { - standardized and comparable model } \\
\text { performance metrics } \\
\text { - generalizable model classification or } \\
\text { 'fingerprint' } \\
\text { - link differences between models' } \\
\text { structure and parameterization to } \\
\text { differences in models' behavior } \\
\text { - insights on structural validity }\end{array}$ & $\begin{array}{l}\text { - descriptive indicators of model behavior, } \\
\text { not explanatory } \\
\text { - risk of over-tuning to harmonize } \\
\text { diagnostic model runs } \\
\text { - not appropriate or possible for all models } \\
\text { with different designs }\end{array}$ \\
\hline $\begin{array}{l}\text { sensitivity } \\
\text { analysis }\end{array}$ & $\begin{array}{l}\text { - identifies influential inputs and } \\
\text { assumptions } \\
\text { - links model inputs and } \\
\text { parameterization to model behavior }\end{array}$ & $\begin{array}{l}\text { - does not address structural uncertainty in } \\
\text { models } \\
\text { - computational cost of global methods } \\
\text { - limited insights from local methods }\end{array}$ \\
\hline $\begin{array}{l}\text { documentation, } \\
\text { checks, review }\end{array}$ & $\begin{array}{l}\text { - third party verification \& expert } \\
\text { review } \\
\text { - transparency, openness }\end{array}$ & $\begin{array}{l}\text { - costly (time, capacity, intellectual } \\
\text { property) }\end{array}$ \\
\hline
\end{tabular}

Aunque los métodos utilizados por los modelos existentes en la literatura son muy diferentes, el más común es el denominado "proyectos de comparación de modelos (MIPs)", algunos ejemplos de MIPs son EMF33 (Bauer et al., 2018), CD-LINKS (McCollum et al., 2018b; Roelfsema et al., 2020), ADVANCE (Luderer et al., 2018; Vrontisi et al., 2018) o AMPERE (Kriegler et al., 2015). Uno de los grandes problemas de las comparaciones de modelos es que suelen utilizar siempre los mismos modelos, y se corre el riesgo, como se dice en la Tabla 5.1, de que los resultados obtenidos sean siempre de modelos con una línea de pensamiento muy parecida, olvidándose de los modelos con resultados atípicos.

Además de los MIPs, los modelos descritos en la ¡Error! No se encuentra el origen de la referencia. de esta tesis utilizan, en mayor o menor medida, otros métodos de validación. Este es el caso del modelo AIM (Fujimori et al., 2017), que ha realizado pruebas de validación del comportamiento hindcasting en las que compara en distintas partes del modelo los resultados obtenidos en su modelo con los datos históricos (Fujimori et al., 2016). También se ha utilizado este método de validación en el modelo GCAM (Calvin et al., 2020) por ejemplo en la parte 
relativa al módulo del uso del suelo (Calvin et al., 2017). Por otra parte, el modelo REMIND (Aboumahboub et al., 2020) ha realizado análisis de sensibilidad a distintas partes del modelo, como por ejemplo a las energías renovables variables (Ueckerdt et al., 2017)

Sin embargo, pese a los esfuerzos en los últimos años por establecer unos criterios comunes para la validación de los modelos, y pese a las recomendaciones por utilizar y documentar los diferentes métodos, actualmente sigue sin haber un protocolo claro sobre cómo realizar la validación, y las distintas pruebas de validación realizadas a los modelos son escasas o no están documentadas en la literatura. Mientras tanto, cada vez son más los artículos e informes donde se cuestionan la validación de los modelos y las malas prácticas existentes en ellos (Saltelli et al., 2020) y en los que se siguen proponiendo nuevos métodos y alternativas para la validación de los IAM (Eker et al., 2019; Hamilton et al., 2019).

\subsection{Validación de MEDEAS}

En el desarrollo de MEDEAS se ha considerado la validación como el conjunto de pruebas que sirven para mostrar la utilidad de un modelo con el objetivo de resolver un problema. Entre las dos principales escuelas filosóficas planteadas en el apartado anterior, se ha adoptado una visión relativista/holística de la validación, alejándose de la dualidad de modelo correcto e incorrecto. Se ha asumido, por tanto, que no se puede conseguir una objetividad absoluta en el modelo y, a través de las pruebas de validación, se intenta generar una gran confianza en los resultados que este proporciona. Siguiendo esta escuela de pensamiento, el término que se ha utilizado para comprobar la utilidad del modelo es el de "validación", en vez del término menos conflictivo "evaluación" utilizado en otros IAM.

El modelo MEDEAS-W se ha elaborado en los últimos cuatro años siguiendo el proceso lógico de modelado en el que las pruebas de validación se van realizando a la vez que se va desarrollando el modelo. MEDEAS-W es un modelo desarrollado en dinámica de sistemas, por lo que se han realizado las principales pruebas que esta metodología establece (Barlas, 1996; Forrester and Senge, 1980; Schwaninger and Groesser, 2020; Sterman, 2000). Para ello se han utilizado las herramientas disponibles en el software utilizado para el desarrollo del modelo: Vensim DSS 7.2.

En las siguientes páginas se documentan las principales pruebas de validación que se han realizado al modelo durante su proceso de desarrollo. Las pruebas han sido clasificadas en cuatro tipos distintos: pruebas directas de estructura, pruebas indirectas de estructura (corresponden a las llamadas por Barlas pruebas de comportamiento orientadas a la estructura), pruebas de patrones de comportamiento y prueba de mejora del sistema.

Es importante tener en cuenta que el modelo ha ido evolucionado a lo largo del proceso de desarrollo, por lo que las pruebas de validación se han realizado con distintas versiones del modelo MEDEAS-W. En cada prueba de validación que implique una simulación se dirá la versión del modelo que se ha utilizado. Salidas del modelo como el PIB o las emisiones de $\mathrm{CO}_{2}$ pueden ser ligeramente diferentes en las distintas pruebas de validación, ya que han podido utilizar una versión distinta del modelo con pequeñas diferencias en su estructura. Todas las simulaciones realizadas para las pruebas de validación siguen un escenario Business as Usual (BAU) en el que se mantienen las principales tendencias económicas, energéticas y medioambientales. La descripción de las principales hipótesis y valores que caracterizan la narrativa BAU en MEDEASW se han explicado en el Appendix A.1 de esta tesis. 


\subsubsection{Pruebas directas de estructura}

Las pruebas directas de estructura evalúan la validez de la estructura del modelo a través de una comparación directa con el conocimiento existente sobre la estructura del sistema real (Barlas, 1996). En este tipo de pruebas se evalúa la estructura sin examinar el comportamiento del sistema, no implican una simulación del modelo. Ejemplos de estas pruebas son la prueba de la confirmación de la estructura, la prueba de confirmación de los parámetros, la prueba de adecuación de los límites del sistema o la prueba de consistencia en las dimensiones. Estas pruebas se pueden clasificar como empíricas o teóricas según se comparen con la información obtenida del sistema real o del conocimiento general en la literatura respectivamente.

Las pruebas directas de estructura se realizan en el proceso de desarrollo del modelo comprobando sus parámetros, ecuaciones y unidades. En MEDEAS-W se han realizado estas pruebas a todos los subsistemas del modelo permitiendo corregir errores y realizar modificaciones en la estructura. Mostrar en esta tesis las pruebas directas de estructura es extremadamente difícil debido a su naturaleza iterativa y a la gran complejidad del modelo MEDEAS-W v2.0, compuesto por miles de variables y ecuaciones.

Para mostrar el proceso de validación con las pruebas directas de estructura, esta tesis se va a centrar en la parte relativa a la demanda de energía a través de las intensidades energéticas, ya que es un submódulo que ocupa una parte importante en el desarrollo de la tesis (capítulo 4). El resto de partes del modelo han sido igualmente validados con las pruebas directas de estructura.

\section{Confirmación de la estructura}

La prueba de confirmación de la estructura consiste en comparar la estructura del modelo directamente con la estructura del sistema real que el modelo representa. También puede realizarse como una prueba teórica, comparando las ecuaciones del modelo con los conocimientos generales de la literatura. Para superar esta prueba, la estructura del modelo no debe contradecir el conocimiento sobre la estructura del sistema real. En la mayoría de los casos, la prueba de verificación de la estructura se realiza primero sobre la base de los conocimientos personales del desarrollador del modelo, y luego se amplía para incluir las críticas de otras personas con experiencia directa en el sistema real (Forrester and Senge, 1980).

Las pruebas de confirmación de la estructura son quizás las más difíciles de formalizar y cuantificar. La información necesaria para este tipo de pruebas es de naturaleza altamente cualitativa, no se puede obtener por un conjunto de datos numéricos. Un ejemplo de prueba de confirmación de la estructura es la realizada sobre las variables que físicamente nunca pueden ser negativas. Esto significa que debe haber un bucle de realimentación negativa de primer orden de modo que el flujo sea cero cuando el stock sea nulo. Estos bucles deben ser de primer orden porque cualquier retraso en el bucle podría hacer que la tasa continuara reduciendo el stock incluso después de que el stock llegara a cero, lo cual es físicamente imposible. Comprobar la presencia del bucle de primer orden en estas variables se puede hacer mediante la inspección directa de las ecuaciones (Sterman, 2000). Otros ejemplos de pruebas de confirmación de la estructura son los diagramas de subsistema o los diagramas de causalidad.

Para el caso de estudio de la estimación del consumo de energía a través de las intensidades energéticas en MEDEAS-W lo primero que se va a mostrar son los diagramas de causalidad. En la Figura 5.4 se muestra como el consumo de energía depende de la intensidad energética y de las variables económicas, y a su vez se muestra como el consumo de energía está realimentado por 
la escasez energética a partir de tres bucles negativos. Estos tres bucles negativos, como se explica en el capítulo 4 de esta tesis, tienden a estabilizar el modelo cuando hay escasez (de Blas et al., 2019).

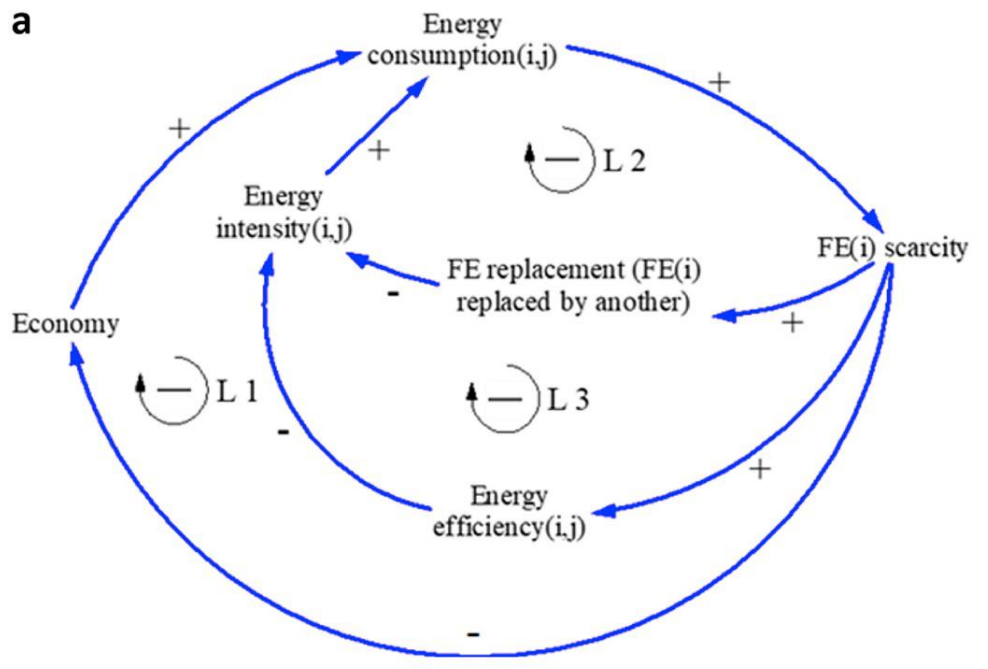

Figura 5.4: Diagrama causal que muestra los efectos de la escasez de una energía final $(i)$ en el marco de los modelos MEDEAS.

Notas: La j representa los 35 sectores económicos considerados en MEDEAS-W.

Fuente: (de Blas et al., 2019).

En la Figura 5.4 también se observa como las intensidades energéticas dependen de la sustitución de un tipo de energía final por otra y de la eficiencia energética. Estas dos fuerzas a su vez dependen de la percepción de la escasez y de políticas externas, como se puede ver en el diagrama causal de la Figura 5.5. Una explicación detallada de esas relaciones se muestra en el trabajo realizado por de Blas et al., (de Blas et al., 2019) y en el capítulo 4 de esta tesis.

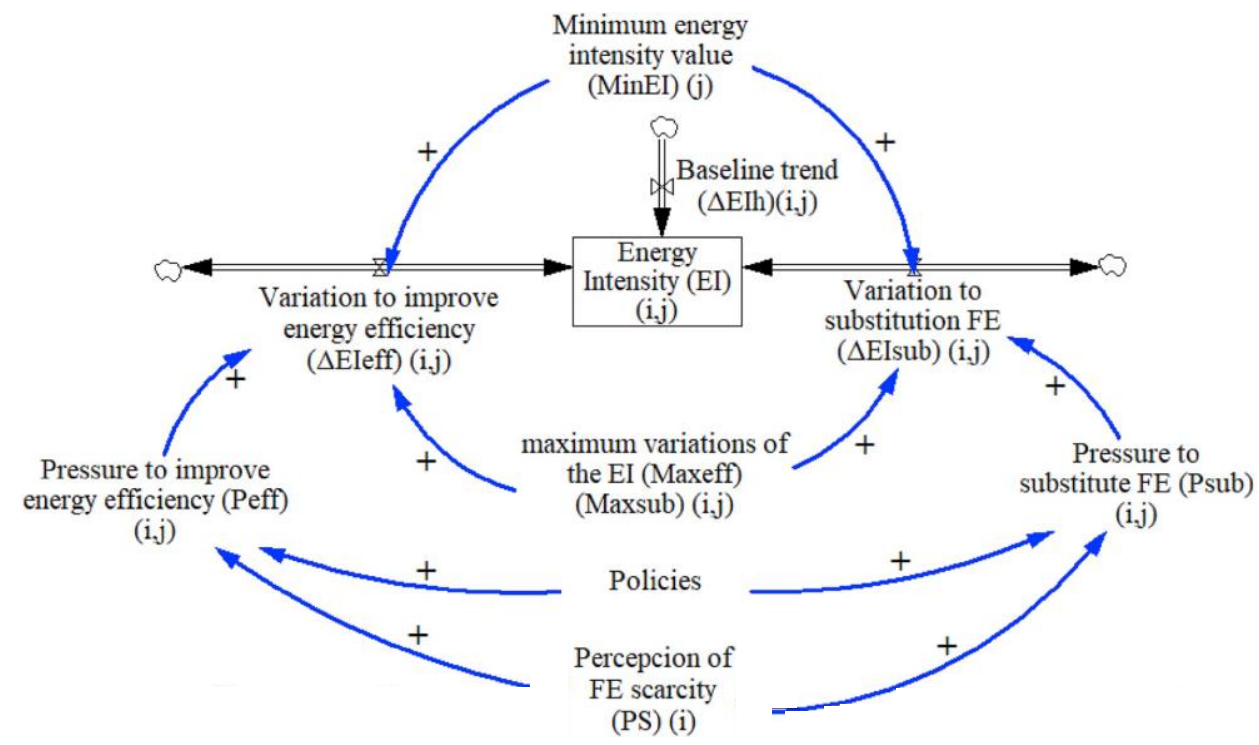

Figura 5.5: Diagrama causal que muestra las principales relaciones de las intensidades energéticas en el marco de los modelos MEDEAS.

Notas: La i representa las energías finales y la j los 35 sectores económicos considerados.

Fuente: (de Blas et al., 2019). 
Como se puede observar en la Figura 5.5, la intensidad energética en los modelos MEDEAS es una variable de estado del sistema (stock) y esta variable, por definición, nunca puede ser menor que cero, las intensidades energéticas no pueden ser negativas. Hay que comprobar si hay un bucle de retroalimentación negativa de primer orden para que las intensidades nunca sean menores que cero.

Un bucle de primer orden es el sistema de realimentación más simple que existe en dinámica de sistemas. Hay una variable de estado (S) que acumula su tasa de entrada neta y, a su vez, la entrada neta depende del estado del sistema, como puede verse en la Figura 5.6 (Sterman, 2000).

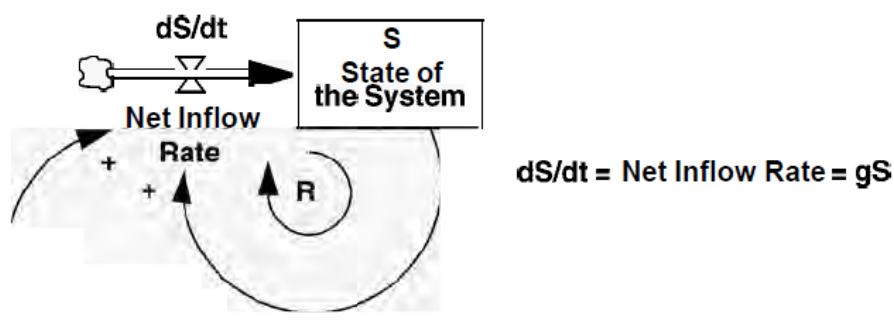

Figura 5.6: Ejemplo de bucle de primer orden. Variable de estado: "S" y flujo: "Net Inflow Rate".

Fuente: Bussines Dynamics (Sterman, 2000).

Con una inspección directa del submódulo de las intensidades energéticas de MEDEAS-W v2.0 se puede observar como los flujos que entran/salen en el stock (intensidad energética) dependen a su vez de esa variable, mostrando el bucle de primer orden y, con ello, que las intensidades energéticas nunca se harán negativas (ver Figura 5.7).

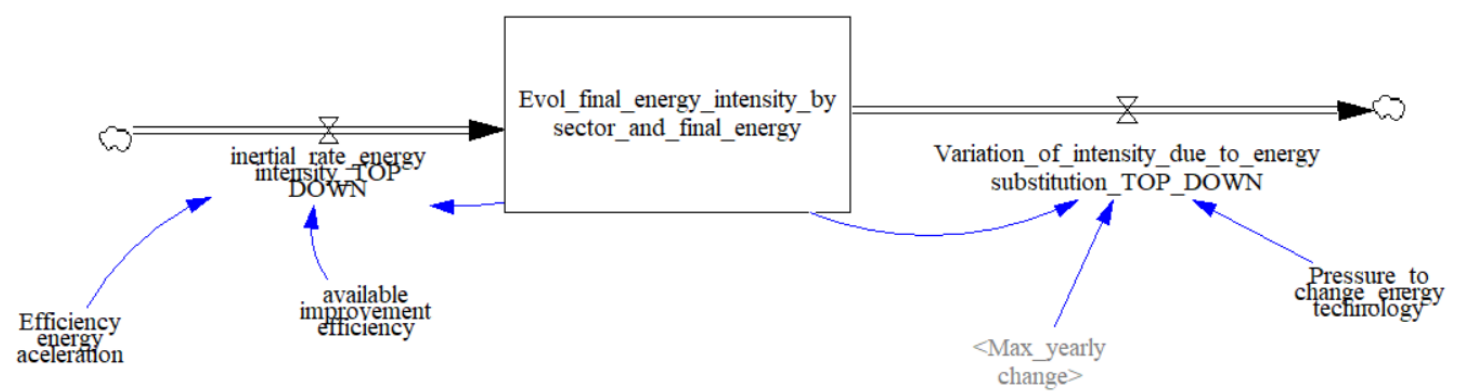

Figura 5.7: Parte del submódulo de las intensidades energéticas en MEDEAS-W v2.0 en el que se muestran los bucles de primer orden entre el stock y sus flujos de entrada y salida.

Fuente: (Capellán-Pérez et al., 2017a).

Analizando las ecuaciones se puede observar como ambas variables dependen del stock, confirmando el bucle de realimentación negativa de primer orden:

- inertial_rate_energy_intensity_TOP_DOWN=Evol_final_energy_intensity_by_secto $\underline{r}$ _and_final_energy*Efficiency_energy_aceleration*available_improvement_efficiency

- Variation_of_intensity_due_to_energy_substitution_TOP_DOWN=Max_yearly_ch ange*Evol final energy intensity by sector and final energy* Pressure_to_change_e nergy_technology

Estos son algunos ejemplos de las muchas pruebas de confirmación de la estructura que se han hecho en todos los submódulos del modelo MEDEAS-W v2.0. Como se ha dicho anteriormente, 
estas son las pruebas más difíciles de formalizar y cuantificar, pero son totalmente necesarias para el correcto desarrollo del modelo.

\section{Confirmación de los parámetros}

La prueba de confirmación de los parámetros consiste en comparar los parámetros del modelo con el conocimiento que se tenga de los parámetros en el sistema real, tanto conceptual como numéricamente (Forrester and Senge, 1980). La confirmación conceptual consiste en ser capaz de identificar los elementos del sistema real que corresponden a los parámetros del modelo, mientras que la confirmación numérica consiste en estimar el valor numérico del parámetro con suficiente precisión (Barlas, 1996).

Antes de decidir cómo estimar un parámetro lo primero es asegurarse de que ese parámetro tiene un significado claro y real. A continuación, el parámetro se puede estimar a través de dos metodologías: mediante estadística formal o mediante conocimiento subjetivo a través de la opinión de expertos obtenido en entrevistas, talleres, material de archivo u otros métodos. En la práctica, ambos métodos se utilizan conjuntamente. El conocimiento del sistema real limita el rango de muchos parámetros; la estimación estadística proporciona una comprobación de las estimaciones con criterio. (Sterman, 2000).

En un modelo grande como MEDEAS-W suele ser poco práctico estimar todos los parámetros críticos simultáneamente, así que una estimación parcial de un subsistema del modelo es muy útil para la selección de parámetros. Al igual que en la prueba anterior se va a mostrar solo la estimación de parámetros del submódulo de intensidades energéticas. Los parámetros exógenos de esta parte del modelo se han obtenido mediante estimaciones estadísticas tomando como referencia los valores históricos, a través del conocimiento de los expertos y también mediante pruebas de calibración.

Un ejemplo es la estimación estadística del parámetro "variación máxima anual de las intensidades energéticas por sector y tipo de energía final" utilizando para ello los datos históricos de la base de datos WIOD entre los años 1995 y 2009 (Dietzenbacher et al., 2013). Se han estimado la variación máxima anual debido a la mejora de eficiencia energética $\left(\Delta \mathrm{EI}^{\mathrm{h} \text { eff }}{ }_{\mathrm{ij}}\right)$ y la variación máxima anual debida a la sustitución entre tipos de energía $\left(\Delta \mathrm{EI}^{\mathrm{h}}{ }^{\mathrm{sub}}{ }_{\mathrm{ij}}\right)$. Las variables $\Delta \mathrm{EI}^{\mathrm{h}}$ eff $_{i j} \mathrm{y} \Delta \mathrm{EI}^{\mathrm{h} \text { sub }}{ }_{\mathrm{ij}}$ se han definido como variables aleatorias con una distribución de probabilidad definida por su valor medio y por su varianza $\mu\left(\left(\Delta \mathrm{EI}^{\mathrm{h} \text { eff }_{i j}}\right), \sigma^{2}\left(\Delta \mathrm{EI}^{\mathrm{h}} \mathrm{eff}_{\mathrm{ij}}\right)\right.$ y $\mu\left(\Delta \mathrm{EI}^{\mathrm{h} \text { sub }_{\mathrm{ij}}}\right), \sigma^{2}\left(\Delta \mathrm{EI}^{\mathrm{h} \text { sub }_{i j}}\right)$. La variación máxima anual para las intensidades energéticas por sector y por tipo de energía final se obtienen a través de las siguientes ecuaciones (eq. 5.1 y eq. 5.2) y se muestran en la ¡Error! No se encuentra el origen de la referencia. y la ¿Error! No se encuentra el origen de la referencia. del Appendix B.1 de esta tesis:

$$
\begin{gathered}
\operatorname{Max}^{\text {eff }}{ }_{j}=\sqrt{\frac{\sigma^{2}\left(\Delta \mathrm{EI}^{e f f}{ }_{j}\right)}{n *(1-\alpha)}} \\
\operatorname{Max}^{s u b}{ }_{i j}=\mu\left(\Delta \mathrm{EI}^{\text {sub }}{ }_{i j}\right)+\sqrt{\frac{\sigma^{2}\left(\Delta \mathrm{EI}^{\text {sub }}{ }_{i j}\right)}{n *(1-\alpha)}}
\end{gathered}
$$

Un ejemplo dentro del submódulo de las intensidades energéticas en el que la estimación se ha realizado basándose en datos de expertos es el parámetro "valor mínimo de la intensidad 
energética por sector". Se ha utilizado un estudio de Ligthfoot and Green (Lightfoot and Green, 2002) en el que analiza las posibles mejoras de eficiencia de los diferentes sectores (electricidad, transporte, residencial, industrial y comercial) hasta el año 2100 considerando mejoras técnicas de eficiencia energética a nivel mundial.

\section{Adecuación de los límites del sistema}

Las pruebas de adecuación de los límites del sistema evalúan la idoneidad de los límites del modelo para el propósito en cuestión (Sterman, 2000). Esta prueba analiza si dentro de los límites se incluye toda la estructura relevante en la modelización del sistema. Al realizar esta prueba hay que tener en cuenta cuál es el propósito del modelo. Si no se hace, los límites del modelo pueden extenderse indefinidamente a medida que se incorporan al modelo otros aspectos de la estructura del sistema real que, aunque sean exactos, no son necesarios para el propósito particular. Entre las herramientas útiles para esta prueba se incluyen las tablas de los límites del modelo y los diagramas de subsistema. Por lo general, en esta prueba hay que plantearse relaciones potencialmente importantes que se hayan omitido en el modelo y que tengan relación con su propósito.

Como en las pruebas anteriores, a continuación se van a mostrar las herramientas utilizadas para la prueba de adecuación de los límites del sistema al submódulo de la demanda de energía en MEDEAS-W (ver ¡Error! No se encuentra el origen de la referencia.). Una tabla de los límites del modelo resume el alcance del mismo enumerando las variables clave que se incluyen de forma endógena, las que son exógenas y las que se excluyen del modelo. En la Tabla 5.2 se muestran los límites del módulo de la demanda de energía en el modelo MEDEAS-W v2.0.

Tabla 5.2: Tabla de los límites del módulo de la demanda de energía en MEDEAS-W v2.0

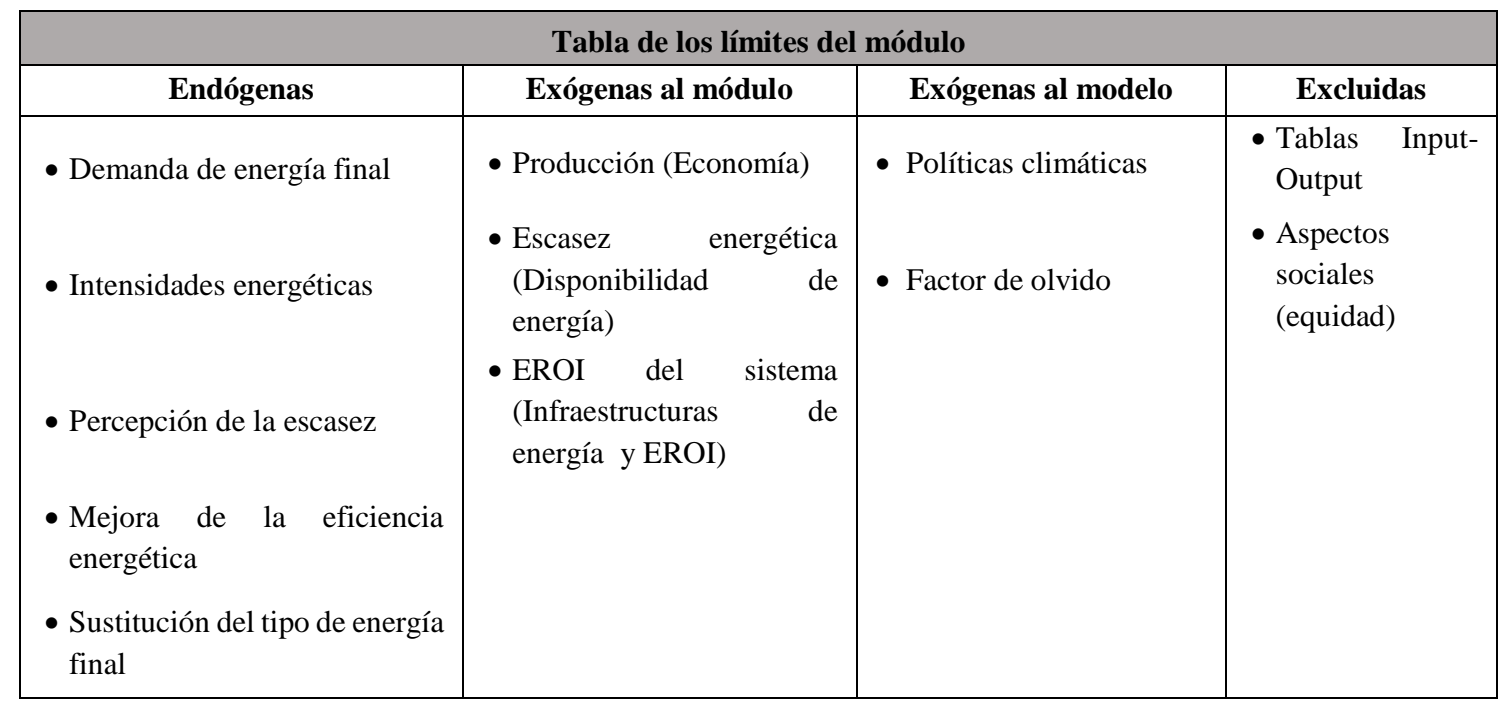

En la tabla anterior se puede observar cómo han quedado excluidas del modelo tanto las tablas input-output del módulo de economía como los aspectos sociales, por ejemplo, la equidad. Sin embargo, las tablas input-output después de debatirlo con expertos se ha llegado a la conclusión de que es necesario que estén dentro de los límites de este submódulo puesto que su relación con las intensidades energéticas en muy importante. No incluir las tablas input-output en este módulo es una de las limitaciones actuales del modelo que se ha descubierto gracias a una prueba de adecuación de los límites. Actualmente se está trabajando en relacionar ambas variables, pero 
como en MEDEAS-W v2.0 no está aún modelada, esta relación queda excluida en la tabla anterior.

Para entender mejor las variables señaladas en la Tabla 5.2, se muestra a continuación el diagrama de subsistema donde quedan reflejados los límites del módulo y sus relaciones con otros módulos (Figura 5.8). Las políticas de cambio climático son totalmente exógenas al modelo, pero como se deciden a partir de los efectos mostrados en el módulo climático se muestran en la figura unidas a ese modulo con una línea de puntos.

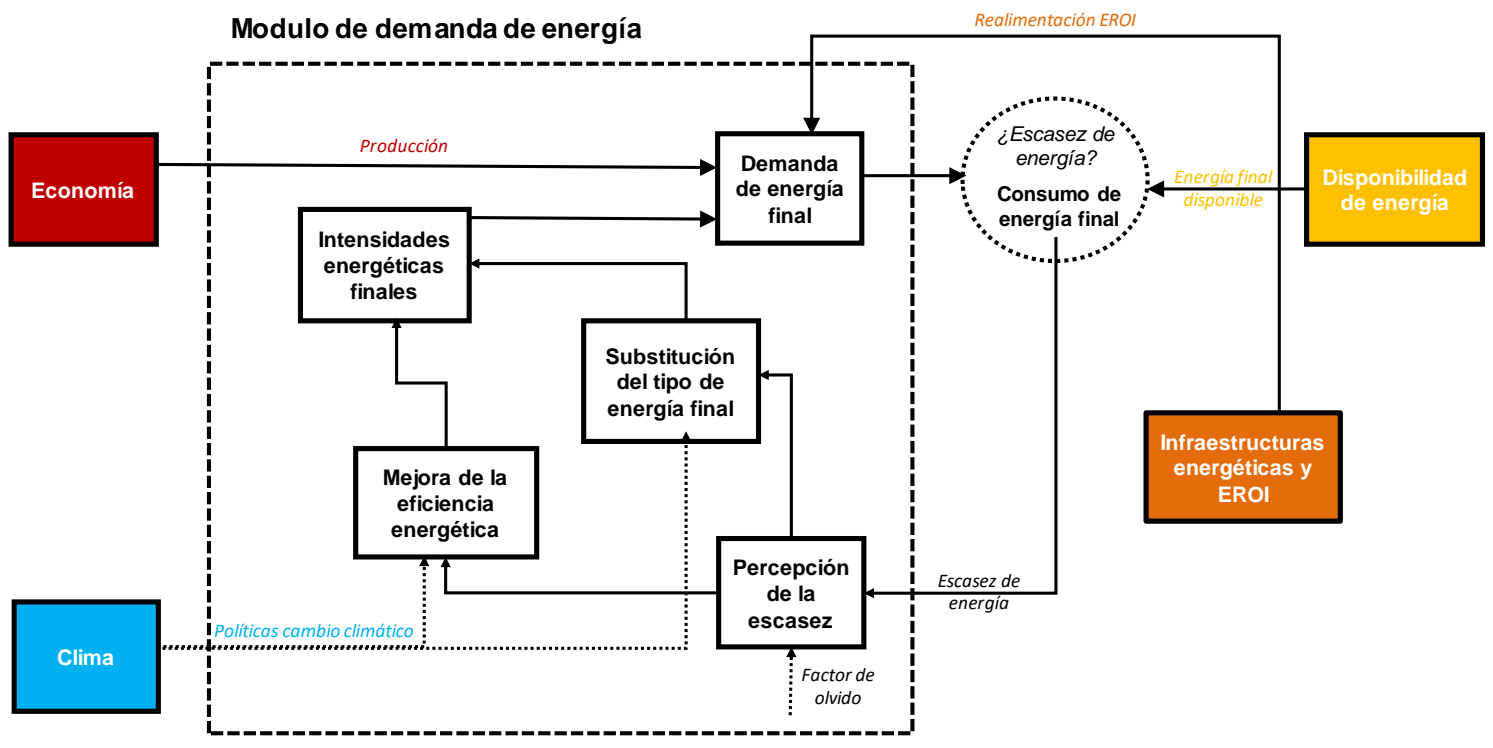

Figura 5.8: Diagrama de subsistema del módulo de la demanda de energía en MEDEAS-W.

Notas: La línea de puntos muestra las variables exógenas al modelo.

El módulo de la demanda de energía es uno de los más "sencillos" del modelo MEDEAS-W en cuanto a número de variables endógenas y relaciones con otros módulos. En el trabajo de Capellán-Pérez et al., que corresponde al capítulo 3 de esta tesis se han mostrado diagramas de subsistemas de otros módulos del modelo (Capellán-Pérez et al., 2020).

\section{Consistencia en las dimensiones}

La prueba de consistencia dimensional consiste en comprobar que las dimensiones de los dos lados de las ecuaciones son iguales (Forrester and Senge, 1980). Los errores en las unidades del modelo suelen revelar importantes fallos en la estructura. Cada ecuación debe ser dimensionalmente consistente sin la inclusión de factores de escala arbitrarios que no tienen significado en el mundo real. La única forma de identificar esos factores de distorsión es mediante la inspección directa de las ecuaciones. Los parámetros con nombres sin sentido, combinaciones extrañas de unidades (personas ${ }^{2} / \$$ años $^{3}$ ), o parámetros no dimensionales con valores de unidad son sospechosos (Sterman, 2000).

Como en todas las pruebas directas de estructura, en esta tesis se van a mostrar, en cuanto a consistencia en las dimensiones, solo los resultados del módulo de la demanda de energía. La demanda de energía se mide generalmente en el modelo MEDEAS-W en EJ ( 1 exajulio $(E J)=$ $10^{18} \mathrm{~J}$ ). Las variables económicas se miden en el modelo en dólares de Estados Unidos del año 1995 (\$ US1995). Las intensidades energéticas, por consiguiente, al ser una división entre una variable energética y una económica, se miden en EJ/T\$, siendo $1 \mathrm{~T} \$=10^{12} \$$. 
Sin considerar la producción económica que ya hemos dicho que se mide en dólares US1995, las otras variables que entran a este módulo desde otras partes del modelo tienen la peculiaridad de ser variables adimensionales (ver Figura 5.8). La escasez de energía se define en MEDEAS como la división entre la demanda de energía no cubierta por la disponibilidad de energía (fes) y la demanda de energía (fed) para cada tipo de energía final "k" como se muestra en la eq. 5.3, por lo que será adimensional (EJ/EJ).

$$
\text { escasez }_{k}=\frac{f e d_{k}-f e s_{k}}{f e d_{k}}
$$

Lo mismo ocurre para el EROI del sistema, que se define como la relación entre la energía final entregada a la sociedad y las inversiones energéticas asociadas a la producción de esa energía. El EROI será entonces también adimensional (EJ/EJ). Siendo dos de las tres variables que entran al módulo adimensionales (ver Figura 5.8), en el módulo de la demanda de energía solo se han considerado variables con unidades energéticas y económicas.

La validación general de la consistencia en las dimensiones del modelo MEDEAS-W v2.0 no es aún satisfactoria completamente. Se han detectado durante el proceso de validación errores formales relacionados especialmente con variables adimensionales que van a ser corregidos en próximas versiones del modelo.

\subsubsection{Pruebas indirectas de estructura}

Las pruebas indirectas de estructura, o también denominadas pruebas de comportamiento orientadas a la estructura, evalúan indirectamente la validez de la estructura aplicando pruebas en patrones de comportamiento generados por los modelos. Este tipo de pruebas implica una simulación del modelo y pueden estar aplicadas a todo el modelo, así como a submodelos del mismo. Ejemplos de estas pruebas son efectuar cambios en el método de integración, realizar una simulación de condiciones extremas o hacer un análisis de sensibilidad e incertidumbre.

\section{Cambios en el método de integración}

Los modelos de dinámica de sistemas suelen formularse en tiempo continuo y resolverse mediante integración numérica, utilizando para simular un tiempo de paso y un método de integración. Los resultados de los modelos no deben ser sensibles ni al tiempo de paso y ni al método de integración seleccionado.

En el modelo MEDEAS-W se han utilizado las opciones que dispone el software Vensim DSS relativas a los métodos de integración y al tiempo de paso. En cuanto a los métodos de integración se pueden utilizar Euler y Runge-Kutta (RK) de $2^{\circ}$ y de $4^{\circ}$ orden con ajuste automático del tamaño de paso o con un paso fijo (RK 2 Auto, RK 4 Auto, RK 2 Fixed y RK 4 Fixed). También permite usar un método por diferencias que realiza la integración de Euler pero almacena los valores de las variables auxiliares calculadas en el tiempo de paso anterior. Respecto al tiempo de paso, en Vensim DSS se puede simular con un tiempo de paso de un año o reducir ese tiempo un número de veces potencia de $2(0.5,0.25,0.125,0.0625,0.03125 \ldots)$.

En la Figura 5.9 y la Figura 5.10 se muestran la evolución del PIB y el cambio en la temperatura global, respectivamente, para simulaciones del modelo MEDEAS-W v2.0, utilizando los seis métodos de integración que dispone Vensim DSS. 
PIB

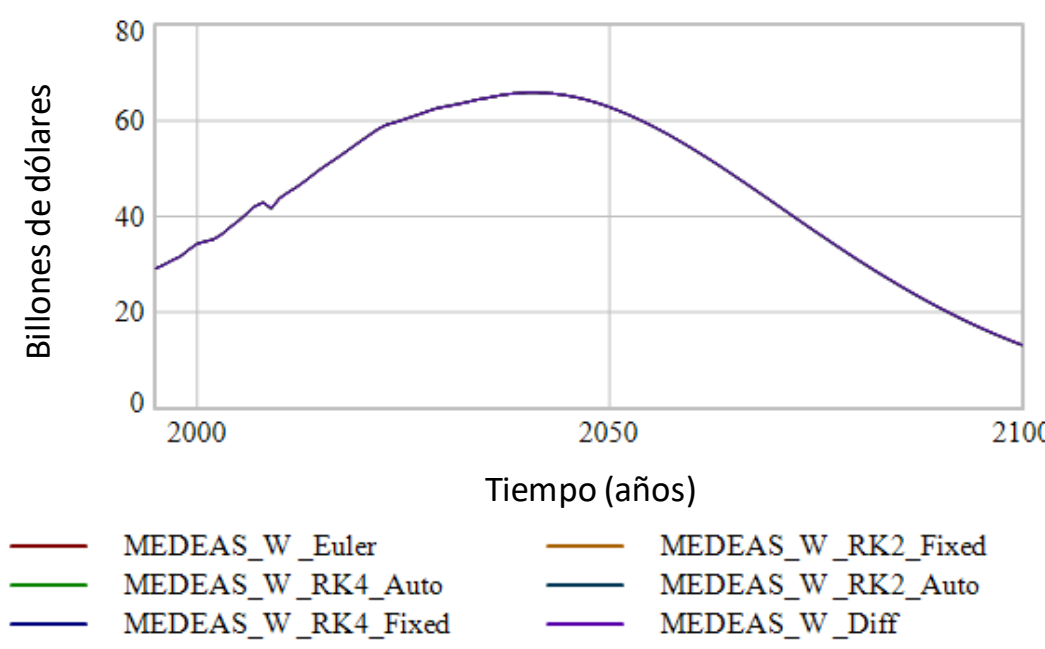

Figura 5.9: Evolución del PIB a nivel mundial en billones de dólares de EEUU del año 1995 utilizando seis métodos de integración distintos en MEDEAS-W 2.0.

Notas: Los métodos de integración utilizados son Euler, RK 2 paso automático, RK 4 paso automático, $R K 2$ paso fjo, $R K 4$ paso fjo y Euler por diferencias.

\section{Cambio en la temperatura}

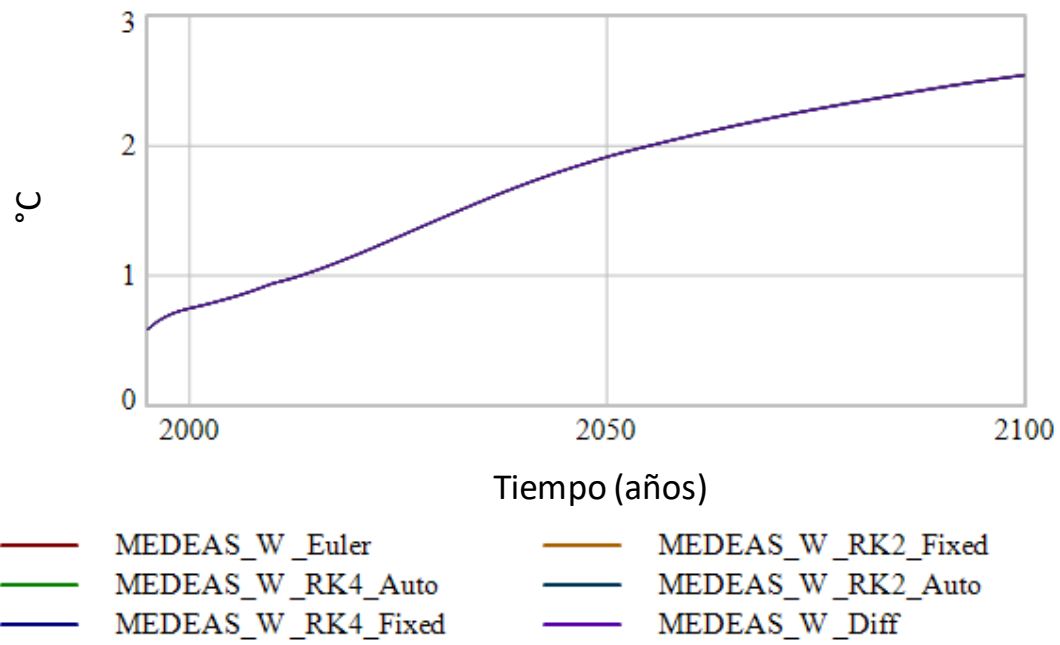

Figura 5.10: Cambio en la temperatura global utilizando seis métodos de integración distintos en MEDEAS-W 2.0.

Notas: Los métodos de integración utilizados son Euler, RK 2 paso automático, RK 4 paso automático, RK 2 paso fjo, RK 4 paso fjo y Euler por diferencias.

La exploración visual muestra que apenas hay diferencia en los resultados obtenidos usando un método de integración u otro. Para una mayor precisión en esta prueba de validación se han analizado las diferencias en los datos año a año para varias salidas del modelo, obteniendo como conclusión que la elección del método de integración no genera, en las variables analizadas, una diferencia superior al 1.5\%. Los resultados para el caso de las dos variables mostradas anteriormente se pueden observar en la Tabla 5.3. 
Tabla 5.3: Valores del PIB y del aumento de la temperatura global en los años 1995, 2050 y 2100 utilizando seis métodos de integración distintos en MEDEAS-W v2.0.

Notas: Los métodos de integración utilizados son Euler, RK 2 paso automático, RK 4 paso automático, RK 2 paso fjo, $R K 4$ paso fjo y Euler por diferencias.

\begin{tabular}{|l|l|l|l|}
\hline PIB (Billones de dólares) & \multicolumn{3}{l}{} \\
\hline Tiempo (años) & 1995 & 2050 & 2100 \\
\hline MEDEAS_W_Euler_0_03125 & 29.15855 & 62.90068 & 13.18703 \\
\hline MEDEAS_W_RK4_Fixed_0_03125 & 29.15855 & 62.90925 & 13.0994 \\
\hline MEDEAS_W_RK4_Auto_0_03125 & 29.15855 & 62.95601 & 13.18871 \\
\hline MEDEAS_W_RK2_Fixed_0_03125 & 29.15855 & 62.90024 & 13.0942 \\
\hline MEDEAS_W_RK2_Auto_0_03125 & 29.15855 & 62.90238 & 13.12114 \\
\hline MEDEAS_W_Diff_0_03125 & 29.15855 & 62.92056 & 13.20749 \\
\hline Diferencia máxima entre simulaciones & $0.000 \%$ & $0.089 \%$ & $0.858 \%$ \\
\hline & & & \\
\hline Aumento de la temperatura (ㅇ) & & & \\
\hline Tiempo (años) & 1995 & 2050 & 2100 \\
\hline MEDEAS_W_Euler_0_03125 & 0.585 & 1.91633 & 2.54722 \\
\hline MEDEAS_W_RK4_Fixed_0_03125 & 0.585 & 1.91493 & 2.54623 \\
\hline MEDEAS_W_RK4_Auto_0_03125 & 0.585 & 1.91625 & 2.54993 \\
\hline MEDEAS_W_RK2_Fixed_0_03125 & 0.585 & 1.91642 & 2.54997 \\
\hline MEDEAS_W_RK2_Auto_0_03125 & 0.585 & 1.91635 & 2.54754 \\
\hline MEDEAS_W_Diff_0_03125 & 0.585 & 1.91575 & 2.54695 \\
\hline Diferencia máxima entre simulaciones & $0.000 \%$ & $0.078 \%$ & $0.147 \%$ \\
\hline
\end{tabular}

También se ha realizado un análisis variando el tiempo de paso de la simulación. Se han probado todas las opciones que permite Vensim DSS $(1,0.5,0.25,0.125,0.0625,0.03125,0.015625 \mathrm{y}$ 0.007825 años) utilizando Euler como método de integración. Las diferencias entre los resultados de estas simulaciones son mínimas, como puede observarse en la Figura 5.11 y la Figura 5.12, que muestran la evolución del PIB y el cambio en la temperatura global, respectivamente, para tiempos de paso entre 0.25 y 0.007825 .

PIB

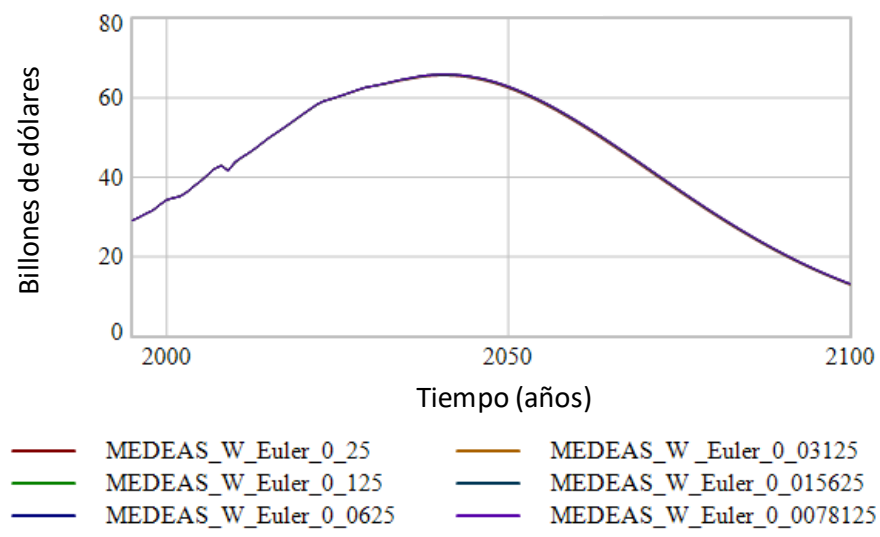

Figura 5.11: Evolución del PIB en billones de dólares de EEUU del año 1995 a nivel mundial utilizando seis tiempos de paso distintos en MEDEAS-W 2.0.

Notas: Los tiempos de paso considerados son 0.25, 0.125, 0.0625, $0.03125,0.015625$ y 0.007825 años. 


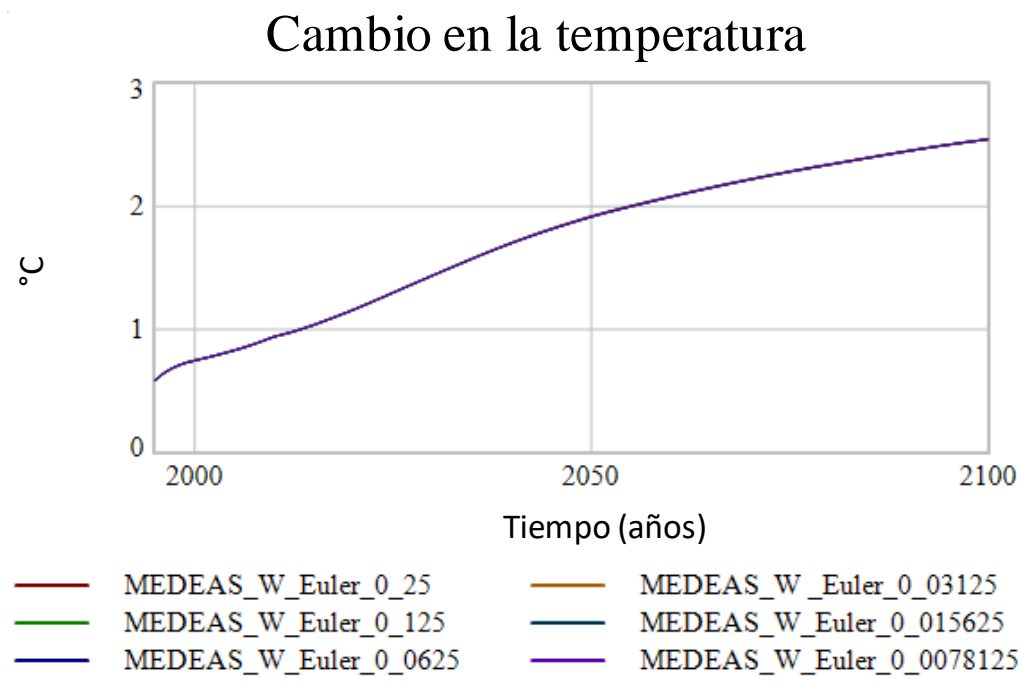

Figura 5.12: Cambio en la temperatura global utilizando seis tiempos de paso distintos en MEDEAS-W 2.0.

Notas: Los tiempos de paso considerados son 0.25, 0.125, 0.0625, $0.03125,0.015625$ y 0.007825 años.

Si analizamos los datos igual que en el caso anterior para ambas variables, vemos que según se va reduciendo el tiempo de paso las diferencias en los resultados entre simulaciones son más pequeñas. Este comportamiento es normal: cuanto más pequeño es el tiempo de paso mayor es la aproximación del método de integración. Para todas las salidas que se han analizado se observa que reducir el tiempo de paso por debajo de 0.03125 años implica un cambio en los resultados del modelo menor del $0.25 \%$. Por ello, buscando un equilibro entre rapidez y precisión en la simulación, se ha establecido como tiempo de paso por defecto en MEDEAS-W el valor 0.03125 años. Usar tiempos de paso más pequeños implicaría aumentar mucho el tiempo de simulación del modelo y, sin embargo, el cambio en los resultados de las variables sería menor del $0.25 \%$. En la Tabla 5.4 se muestran los resultados de cambiar el tiempo de paso de la simulación utilizando Euler como método de integración para dos de las variables analizadas, el PIB y el cambio en la temperatura global.

Tabla 5.4: Valores del PIB y del cambio en la temperatura global en los años 1995, 2050 y 2100 utilizando seis tiempos de paso distintos en MEDEAS-W v2.0.

Notas: Los tiempos de paso considerados son 0.25, 0.125, 0.0625, 0.03125, 0.015625 y 0.007825 años. También se muestra el cambio generado al reducir el tiempo de paso en las simulaciones.

\begin{tabular}{|c|c|c|c|c|}
\hline PIB (Billones de dólares) & & & & $\begin{array}{l}\% \text { cambio al } \\
\text { reducir tiempo de } \\
\text { paso }\end{array}$ \\
\hline Tiempo (años) & 1995 & 2050 & 2100 & \\
\hline MEDEAS_W_Euler_0_25 & 29.15855 & 62.49755 & 12.95388 & - \\
\hline MEDEAS_W_Euler_0_125 & 29.15855 & 62.74212 & 13.08647 & $1.01 \%$ \\
\hline MEDEAS_W_Euler_0_0625 & 29.15855 & 62.85795 & 13.1548 & $0.52 \%$ \\
\hline MEDEAS_W_Euler_0_03125 & 29.15855 & 62.90068 & 13.18703 & $0.24 \%$ \\
\hline MEDEAS_W_Euler_0_015625 & 29.15855 & 62.92099 & 13.20473 & $0.13 \%$ \\
\hline MEDEAS_W_Euler_0_0078125 & 29.15855 & 62.93029 & 13.21569 & $0.08 \%$ \\
\hline Diferencia máxima entre simulaciones & $0.000 \%$ & $0.688 \%$ & $1.981 \%$ & \\
\hline
\end{tabular}




\begin{tabular}{|c|c|c|c|c|}
\hline \multirow{2}{*}{$\begin{array}{l}\text { Aumento de la temperatura }\left({ }^{\circ} \mathrm{C}\right) \\
\text { Tiempo (años) }\end{array}$} & & & & \multirow[t]{2}{*}{$\begin{array}{l}\% \text { cambio al } \\
\text { reducir tiempo de } \\
\text { paso }\end{array}$} \\
\hline & 1995 & 2050 & 2100 & \\
\hline MEDEAS_W_Euler_0_25 & 0.585 & 1.922 & 2.55 & - \\
\hline MEDEAS_W_Euler_0_125 & 0.585 & 1.919 & 2.548 & $0.05 \%$ \\
\hline MEDEAS_W_Euler_0_0625 & 0.585 & 1.917 & 2.548 & $0.03 \%$ \\
\hline MEDEAS_W_Euler_0_03125 & 0.585 & 1.916 & 2.547 & $0.02 \%$ \\
\hline MEDEAS_W_Euler_0_015625 & 0.585 & 1.916 & 2.547 & $0.01 \%$ \\
\hline MEDEAS_W_Euler_0_0078125 & 0.585 & 1.916 & 2.547 & $0.01 \%$ \\
\hline Diferencia máxima entre simulaciones & $0.000 \%$ & $0.312 \%$ & $0.118 \%$ & \\
\hline
\end{tabular}

Es necesario explicar qué ocurre con los tiempos de paso de 1 y 0.5 años que no se han mostrado en las figuras ni en la Tabla 5.4. Simular MEDEAS-W v2.0 con estos tiempos de paso genera una pequeña inestabilidad en el modelo, ya que hay variables de MEDEAS definidas con un tiempo menor que esos tiempos de paso. Un ejemplo de variable con un valor de tiempo menor que 1 año es el tiempo de planificación o construcción de algunas plantas renovables como solares o eólicas.

La conclusión de esta prueba de validación es que el modelo no es sensible a la elección del método de integración y del tiempo de paso. Con la excepción de tiempos de paso superiores a 0.5 años, las diferencias entre los resultados son mínimas. Es importante remarcar que el objetivo del modelo MEDEAS-W, y de los modelos de dinámica de sistemas en general, no es predecir al detalle los resultados de un sistema sino mostrar unos patrones de comportamiento que ayuden a la toma de decisiones, por lo que estas diferencias entre simulaciones son asumibles para este tipo de modelos. Además, los errores en los datos de entrada del modelo pueden ser mayores que los mostrados al cambiar el tiempo de paso.

\section{Simulación de condiciones extremas}

Los modelos deben ser robustos en condiciones extremas, es decir, deben comportarse de manera realista sin importar que las variables de entrada y políticas que se le impongan sean extremas (Sterman, 2000). Por ejemplo, bajo ninguna circunstancia la población puede ser menor que cero o que alguna variable del sistema se vaya a infinito creando un problema computacional. Cuando una simulación de condiciones extremas genera un comportamiento inverosímil, se deben examinar las ecuaciones afectadas para ver cuál es la causa del fallo. Esta prueba consiste en asignar valores extremos a unos parámetros seleccionados y comparar el comportamiento generado en el modelo con el comportamiento real bajo la misma condición extrema (Barlas, 1996). También se le llama prueba de estrés (Balci, 1994).

En esta sección se van a mostrar los resultados de dos pruebas de simulación de condiciones extremas. Primero se muestra una simulación de condiciones extremas en las variables de entrada del modelo realizada a MEDEAS-W v2.0, y a continuación se muestran los resultados de otra prueba de condiciones extremas que han sido publicados sobre el modelo MEDEAS-EU v1.2 (Samsó et al., 2020).

En la prueba de simulación de condiciones extremas realizada a MEDEAS-W v2.0 se han elegido un total de 75 variables correspondientes a las variables exógenas del modelo. Las variables y sus rangos de incertidumbre se muestran en la Tabla 5.5. Estas 75 variables han sido seleccionadas debido a su naturaleza incierta y a que se espera que tengan mucha influencia en las salidas del 
modelo. Ejemplo de ellas son la variación de la población o del PIB per cápita cuya influencia es enorme en prácticamente todos los módulos del modelo, o los potenciales tecno-económicos de las energías renovables, cuyos valores varían en varios órdenes de magnitud según la bibliografía considerada. Los rangos de incertidumbre superiores e inferiores se han estimado para cada grupo de variables intentando cubrir el rango más amplio posible de los valores que permita comprobar la robustez del modelo.

Tabla 5.5: Rangos de incertidumbre de las variable de entrada para el análisis de condiciones extremas en MEDEAS-W $v 2.0$.

\begin{tabular}{|c|c|c|}
\hline Entradas e hipótesis de los escenarios & \multicolumn{2}{|c|}{$\begin{array}{l}\text { Rangos de incertidumbre de las entradas para el } \\
\text { análisis de condiciones extremas (en referencia con los } \\
\text { valores del escenario BAU) }\end{array}$} \\
\hline Población (2015-2100) & \multicolumn{2}{|c|}{ $\pm 70 \%$ del crecimiento de la población en escenario SSP2 } \\
\hline Crecimiento del PIB esperado (2015-2100) & \multicolumn{2}{|c|}{$\begin{array}{l}\text { Valor min: }-5 \% \text { anual, valor max } 20 \% \text { anual (valor del } \\
\text { modelo: } 2 \% \text { ) }\end{array}$} \\
\hline Objetivo de participación del trabajo en el PIB & \multicolumn{2}{|c|}{ Valor min: 0.35 , valor max: 0.7 (valor del modelo: 0.52 ) } \\
\hline \multicolumn{3}{|l|}{ Intensidades energéticas finales } \\
\hline $\begin{array}{l}\text { Intensidad energética final mínima respecto } \\
\text { inicial }\end{array}$ & \multicolumn{2}{|l|}{ $\pm 50 \%$} \\
\hline $\begin{array}{l}\text { \% de cambio sobre la variación histórica } \\
\text { máxima de las intensidades energéticas finales. }\end{array}$ & \multicolumn{2}{|l|}{ $\pm 50 \%$} \\
\hline Factor de olvido de la escasez energética & \multicolumn{2}{|c|}{ Valor min: 1 año, valor max:10 años (modelo: 5 años) } \\
\hline Transporte terrestre y de los hogares & \multicolumn{2}{|c|}{$\begin{array}{l} \pm 100 \% \text { sobre el objetivo del porcentaje de cada tipo de } \\
\text { vehículo entre los años } 2020 \text { y } 2050\end{array}$} \\
\hline $\begin{array}{l}\text { Tasa de reciclado de minerales } \\
\text { (19 minerales) }\end{array}$ & \multicolumn{2}{|c|}{$\begin{array}{l}\text { Mejora del } 0 \%-50 \% \text { anual de la tasa de reciclado entre los } \\
\text { años } 2020 \text { y } 2050\end{array}$} \\
\hline Capacidad nuclear & \multicolumn{2}{|c|}{$\begin{array}{l}\text { Variación de la capacidad nuclear de -50-50\%/año entre } \\
\text { los años } 2020 \text { y } 2050\end{array}$} \\
\hline \multicolumn{3}{|c|}{ Curvas de agotamiento de las energías no renovables } \\
\hline Petróleo & \multicolumn{2}{|c|}{ 0-100\% para la URR y el nivel de extracción máxima } \\
\hline Gas Natural & \multicolumn{2}{|c|}{ 0-100\% para la URR y el nivel de extracción máxima } \\
\hline Carbón & \multicolumn{2}{|c|}{ 0-100\% para la URR y el nivel de extracción máxima } \\
\hline Uranio & \multicolumn{2}{|c|}{ 0-100\% para la URR y el nivel de extracción máxima } \\
\hline $\begin{array}{l}\text { Emisiones de GEI de otros gases que no son } \mathrm{CO}_{2} \\
\text { and } \mathrm{CH}_{4}\end{array}$ & \multicolumn{2}{|c|}{$\begin{array}{l}50 \% \text { probabilidad RCP6.0 } \\
50 \% \text { probabilidad RCP8.5 }\end{array}$} \\
\hline Impactos del cambio climático & \multicolumn{2}{|c|}{$\begin{array}{l}50 \% \text { prob. de la función de daño calibrada a }+1.75^{\circ} \mathrm{C} \text { con } \\
\text { unas pérdidas del PIBpc del } 1 \% \\
50 \% \text { prob. de la función de daño calibrada a }+1.75^{\circ} \mathrm{C} \text { con } \\
\text { unas pérdidas del PIBpc del } 3 \%\end{array}$} \\
\hline \multicolumn{3}{|l|}{ Energías renovables } \\
\hline & $\begin{array}{l}\text { Capacidad crecimiento } \\
\text { anual }(2015-2100)\end{array}$ & Potencial técno-sostenible \\
\hline $\begin{array}{l}\text { Todas las tecnologías para generación de } \\
\text { electricidad }\end{array}$ & Min: $0 \%$ - Max: $30 \%$ & $/ 10-\mathrm{x} 10$ \\
\hline Todas las tecnologías para generación de calor & Min: $0 \%$ - Max: $30 \%$ & $/ 10-\mathrm{x} 10$ \\
\hline Bioenergía convencional & - & $/ 10-\mathrm{x} 10$ \\
\hline Bioenergía de campos de cultivo de $2^{a}$ generación & Min: $0 \%$ - Max: $30 \%$ & \multirow{2}{*}{$/ 10-x 10$} \\
\hline Bioenergía de campos de cultivo de $3^{\text {a }}$ generación & Min: $0 \%$ - Max: $30 \%$ & \\
\hline Bioenergía de residuos & Min: $0 \%$ - Max: $30 \%$ & $/ 10-x 10$ \\
\hline Bioenergía de tierras marginales & Min: $0 \%$ - Max: $30 \%$ & $10-\mathrm{x} 10$ \\
\hline
\end{tabular}

Para realizar esta prueba de simulación de condiciones extremas se ha utilizado la herramienta de Vensim DSS para opciones de sensibilidad. Se han ejecutado 1000 simulaciones de Monte Carlo tomando como muestra distribuciones uniformes entre los valores mínimos y máximos para cada 
parámetro. Los resultados de esta prueba se han analizado cualitativamente utilizando gráficas que muestran los intervalos de confianza en torno a la media de la distribución para cada variable de salida. Las variables de salida sobre las que se evalúa esta prueba se han elegido con el objetivo de analizar variables que abarquen al máximo posible de sistemas del modelo. Por razones prácticas en esta tesis solo se muestran los gráficos de seis de las variables de salida. La Figura 5.13 y la Figura 5.14 presentan los intervalos de confianza en torno a la media de la distribución de las seis variables de salida para las 1000 simulaciones realizadas. La línea roja corresponde al valor de la variable obtenida con los valores nominales de entrada (escenario BAU). 

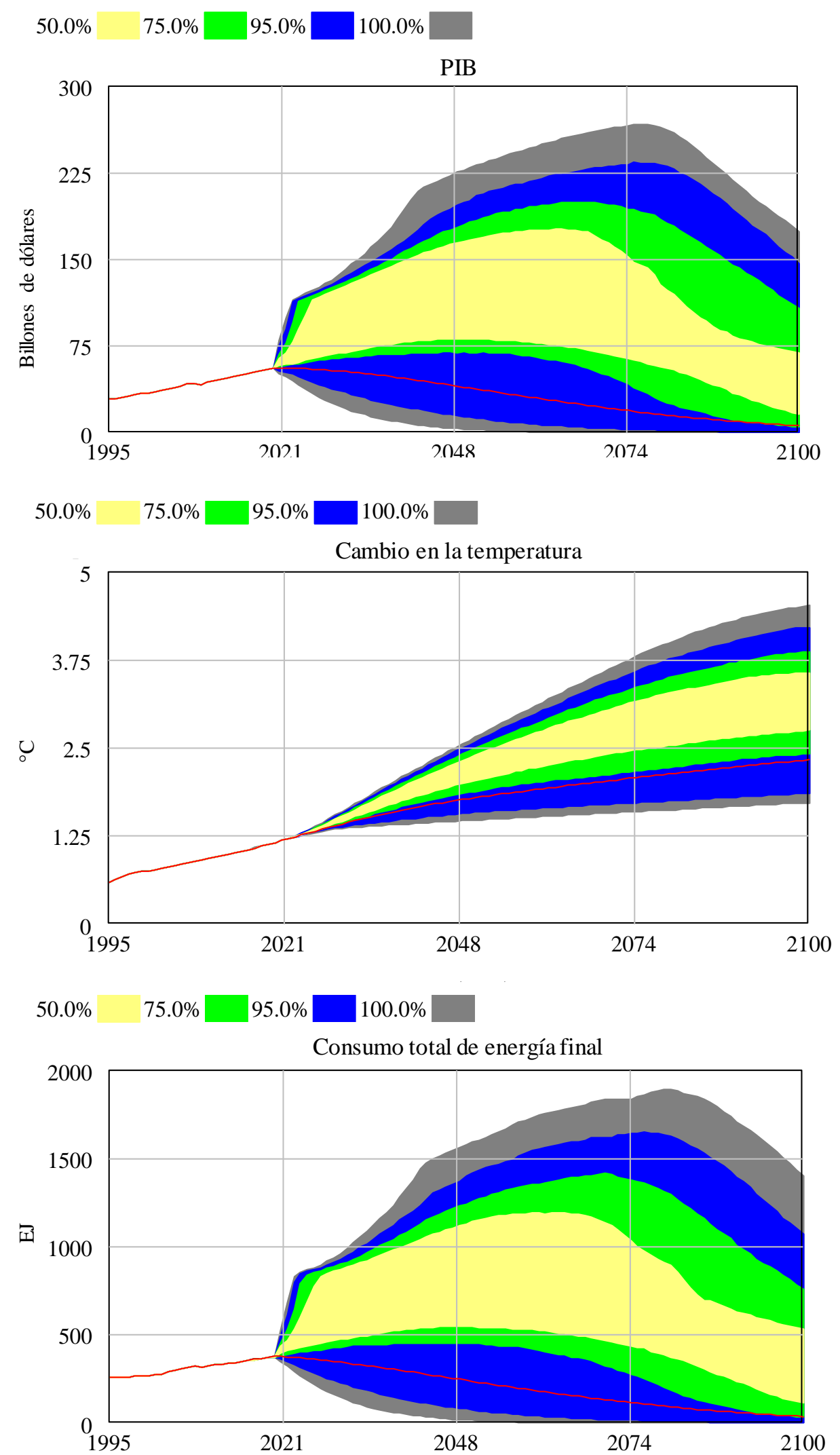

Figura 5.13: Intervalos de confianza del PIB, del aumento de la temperatura global y del consumo total de energía final a nivel mundial bajo las condiciones extremas de la Tabla 5.5 en el modelo MEDEAS-Wv2.0.

Nota: PIB en dólares de EEUU del año 1995. 

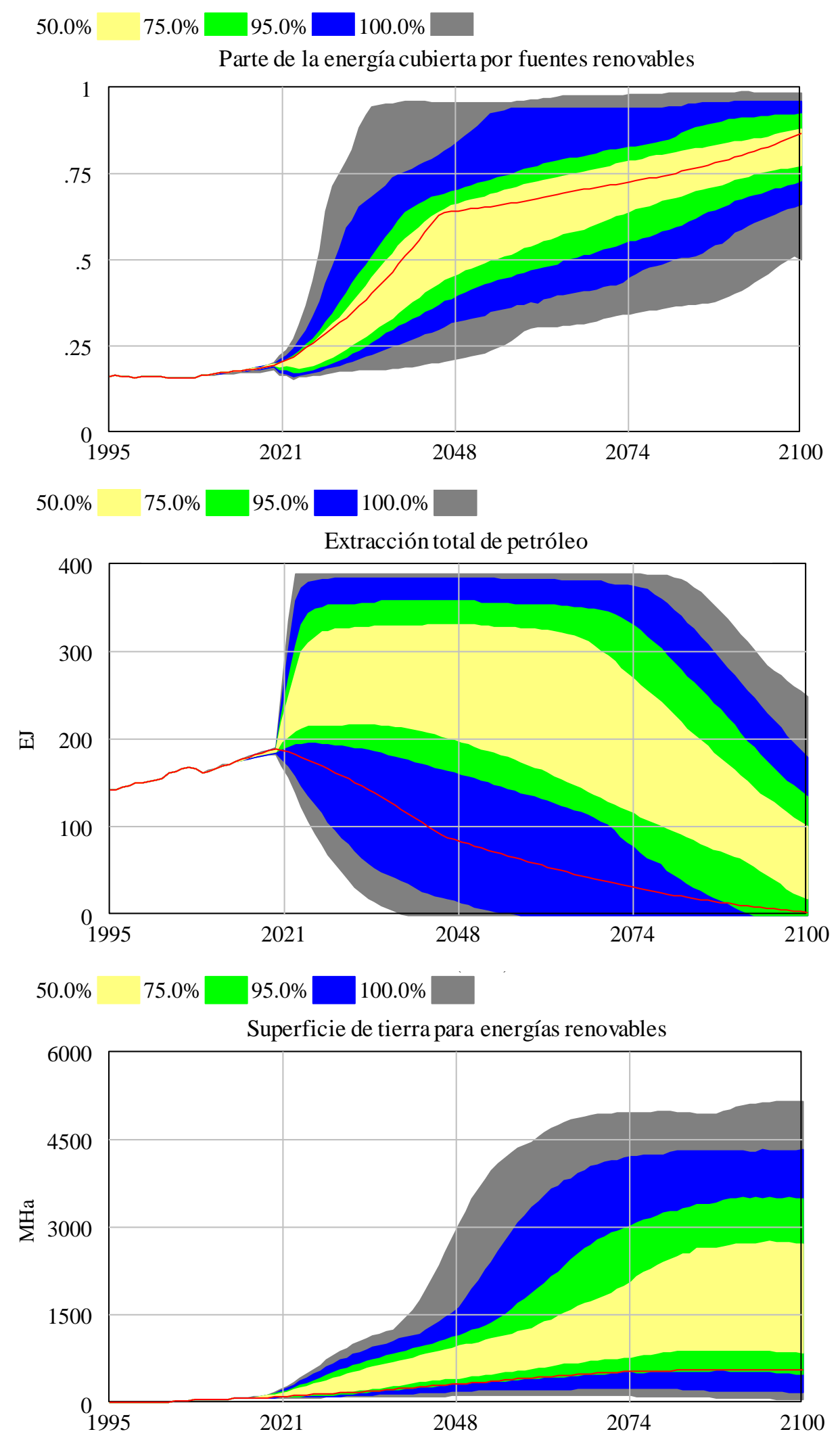

Figura 5.14: Intervalos de confianza de la parte de la energía cubierto por fuentes renovables, de la extracción total de petróleo y de la superficie de tierra necesaria para energías renovables a nivel mundial bajo las condiciones extremas de la Tabla 5.5 en el modelo MEDEAS-W v2.0. 
Los resultados muestran que ninguna de las 1000 simulaciones de Monte Carlo realizadas ha fallado en su intento de converger y que en las variables de salida analizadas se obtienen unos resultados coherentes con el comportamiento real bajo las mismas condiciones extremas. Por lo tanto, la principal conclusión de esta prueba es que, para los valores extremos considerados en la Tabla 5.5, el modelo es estable y robusto.

Como se dijo anteriormente, los resultados de una prueba de condiciones extremas sobre variables de entrada en el modelo MEDEAS-EU v1.2 han sido publicados en Energy Strategy Reviews (Samsó et al., 2020). Aunque por defecto en todas las pruebas de validación nos estamos centrando en el modelo MEDEAS-W, es interesante mostrar en esta tesis que se han realizado pruebas de validación a otros miembros del conjunto de modelos MEDEAS y los resultados han sido también muy positivos. En el modelo MEDEAS-EU v1.2 se ha realizado el análisis de condiciones extremas a 27 variables de entrada utilizando también 1000 simulaciones de un análisis de Monte Carlo. La Figura 5.15 y la Figura 5.16 muestran dos ejemplos de los resultados obtenidos en esta prueba. La explicación de estos resultados se puede encontrar en el trabajo de Samsó (Samsó et al., 2020). Al igual que en el caso anterior, lo más relevante de esta prueba es que el modelo MEDEAS-EU es estable bajo condiciones extremas.

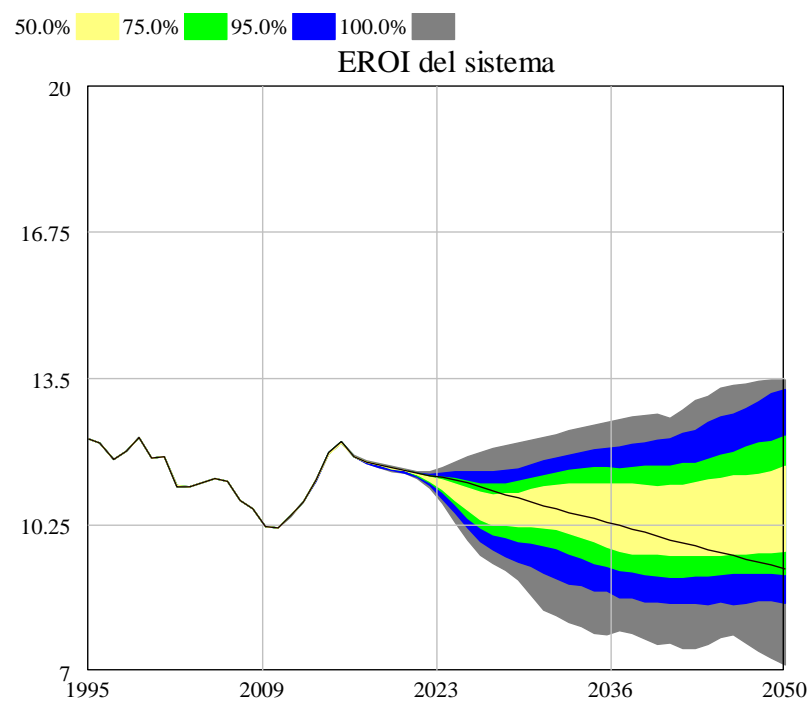

Figura 5.15: Intervalos de confianza para el EROI estándar del sistema tras las 1000 simulaciones del análisis de Monte Carlo en el modelo MEDEAS-EU v1.2. 

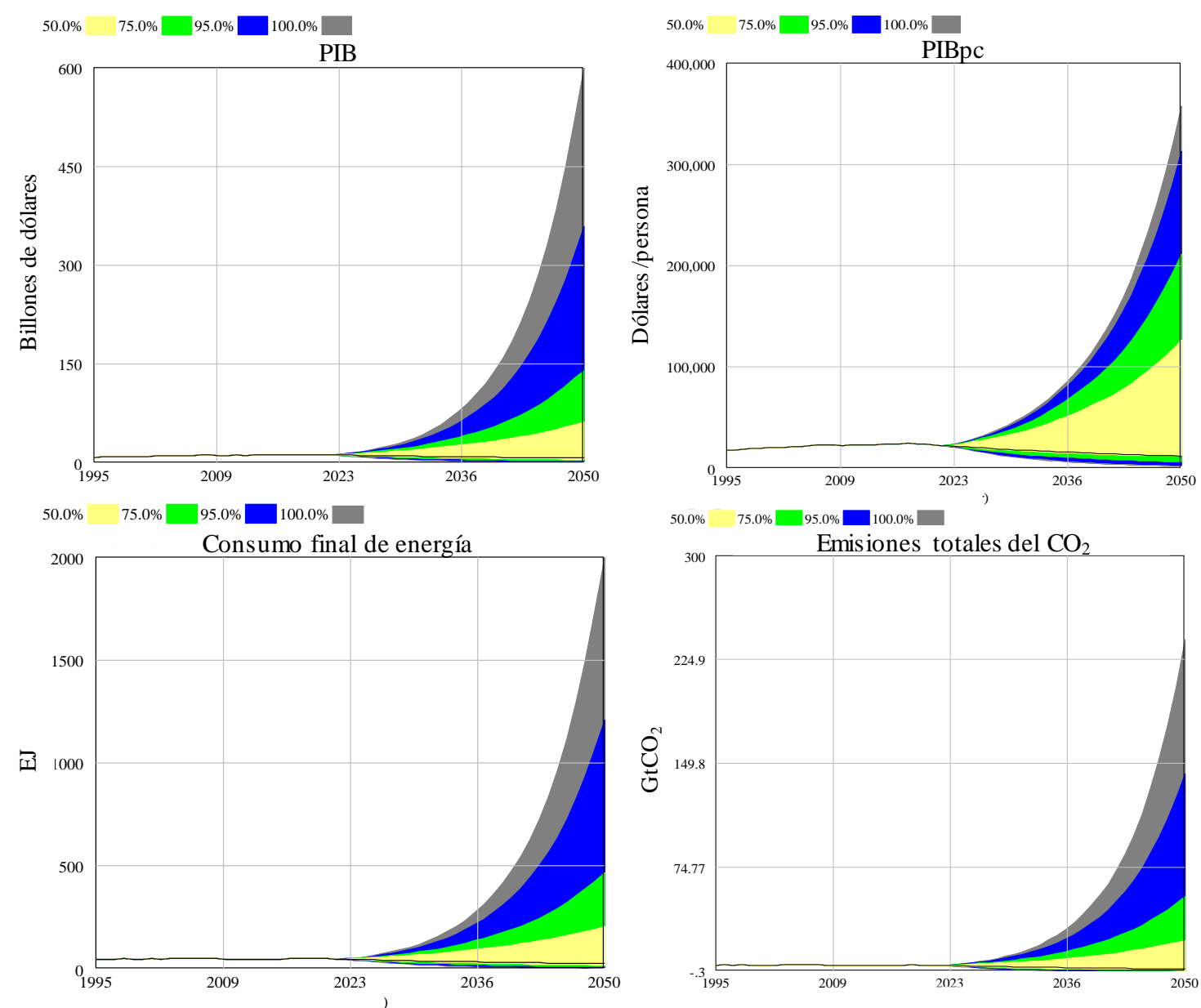

Figura 5.16: Intervalos de confianza para las variables PIB, PIB per cápita, consumo final de energía total y emisiones totales de $\mathrm{CO}_{2}$ tras las 1000 simulaciones del análisis de Monte Carlo en el modelo MEDEAS-EU v1.2.

Nota: PIB en dólares de EEUU del año 1995.

\section{Análisis de sensibilidad}

Las pruebas de análisis de sensibilidad del comportamiento consisten en determinar los parámetros internos a los que el modelo es extremadamente sensible (Barlas, 1996). Aunque por defecto en todas las pruebas de validación nos estamos centrando en el modelo MEDEAS-W, en este caso se van a mostrar los resultados obtenidos al realizar esta prueba al modelo MEDEASEU v1.2. a través de su versión en Python (pymedeas_eu 0.3.0). Este análisis de sensibilidad está documentado en el trabajo realizado por Samsó et al., (Samsó et al., 2020).

Para el análisis de sensibilidad se han seleccionado 19 parámetros del modelo cuyo valor está basado en valores de la literatura, pero es bastante incierto a juicio de los desarrolladores del modelo. Para esta prueba se han estimado unos valores máximos y mínimos de estos parámetros según la opinión de los modeladores basándose en la información de la literatura. Los 19 parámetros se presentan en la Tabla 5.6 obtenida de Samsó et al., (Samsó et al., 2020). 
Tabla 5.6: Nombres de los parámetros exógenos en pymedeas_eu 0.3.0 utilizados para el análisis de sensibilidad junto con su descripción, sus unidades y sus valores nominales, mínimos y máximos.

Fuente: (Samsó et al., 2020).

\begin{tabular}{|c|c|c|c|c|c|}
\hline $\begin{array}{l}\text { Nombre del parámetro } \\
\text { exógeno en Pymedeas }\end{array}$ & Descripción & Unidad & $\begin{array}{l}\text { Valor } \\
\text { nom. }\end{array}$ & $\begin{array}{l}\text { Valor } \\
\text { max. }\end{array}$ & $\begin{array}{l}\text { Valor } \\
\text { min. }\end{array}$ \\
\hline a1_coef_th & $\begin{array}{l}\text { Promedio de energía gastada en el } \\
\text { transporte de los hogares por vehículos } \\
\text { convencionales de cuatro ruedas dividido } \\
\text { por unidad de demanda económica de los } \\
\text { hogares. Se asume su uso y eficiencia } \\
\text { técnica actual. }\end{array}$ & $\begin{array}{l}\mathrm{EJ} / 10^{12} \\
1995 \\
\mathrm{US} \$\end{array}$ & 1.929 & 2.8935 & 0.9645 \\
\hline a2_coef_th & $\begin{array}{l}\text { Promedio de energía gastada en el } \\
\text { transporte de los hogares por vehículos } \\
\text { convencionales de dos ruedas dividido por } \\
\text { unidad de demanda económica de los } \\
\text { hogares. Se asume su uso y eficiencia } \\
\text { técnica actual. }\end{array}$ & $\begin{array}{l}\mathrm{EJ} / 10^{12} \\
1995 \\
\mathrm{US} \$\end{array}$ & 0.5502 & 0.8253 & 0.2751 \\
\hline $\begin{array}{l}\text { eolxdashxrr_minerals_alt } \\
\text { _techn_res_vsx_total_econ } \\
\text { omy }\end{array}$ & $\begin{array}{l}\text { Tasa de reciclado de los minerales } \\
\text { utilizados en las tecnologías alternativas } \\
\text { en relación con la tasa de reciclaje total de } \\
\text { la economía. }\end{array}$ & $\begin{array}{l}\text { Adimen } \\
\text { sional }\end{array}$ & 0.3333 & 1.0 & 0.0 \\
\hline $\begin{array}{l}\text { esoi_phs_depleted_potenti } \\
\text { al }\end{array}$ & $\begin{array}{l}\text { ESOI del potencial usado de PHS. El ESOI } \\
\text { disminuye linealmente con la capacidad } \\
\text { instalada de PHS. }\end{array}$ & $\begin{array}{l}\text { Adimen } \\
\text { sional }\end{array}$ & 5.0 & 10.0 & 1.0 \\
\hline $\begin{array}{l}\text { exponent_availability_con } \\
\text { v_gas }\end{array}$ & $\begin{array}{l}\text { Prioridad del gas convencional sobre el no } \\
\text { convencional. Cuanto menor sea el valor, } \\
\text { mayor será la prioridad del gas } \\
\text { convencional. }\end{array}$ & $\begin{array}{l}\text { Adimen } \\
\text { sional }\end{array}$ & 0.25 & 1.0 & 0.1 \\
\hline $\begin{array}{l}\text { exponent_availability_con } \\
\text { v_oil }\end{array}$ & $\begin{array}{l}\text { Prioridad del petróleo convencional sobre } \\
\text { el no convencional. Cuanto menor sea el } \\
\text { valor, mayor será la prioridad del petróleo } \\
\text { convencional. }\end{array}$ & $\begin{array}{l}\text { Adimen } \\
\text { sional }\end{array}$ & 0.25 & 1.0 & 0.1 \\
\hline $\begin{array}{l}\text { future_share_gasxdivxxco } \\
\text { alxplusxgasx_for_elec }\end{array}$ & $\begin{array}{l}\text { Parte de la electricidad cubierta por gas } \\
\text { natural en relación al cubierto por gas } \\
\text { natural y carbón conjuntamente. }\end{array}$ & $\begin{array}{l}\text { Adimen } \\
\text { sional }\end{array}$ & 0.3 & 1.0 & 0.0 \\
\hline $\begin{array}{l}\text { max_share_transmxandxd } \\
\text { istr_elec_losses }\end{array}$ & $\begin{array}{l}\text { Porcentaje máximo de pérdidas de } \\
\text { transmisión y distribución de electricidad } \\
\text { (cuando las energías renovables } \\
\text { suministran el } 100 \% \text { del consumo). }\end{array}$ & $\begin{array}{l}\text { Adimen } \\
\text { sional }\end{array}$ & 0.1698 & 0.5094 & 0.0566 \\
\hline min_cp_nuclear & $\begin{array}{l}\text { Factor de capacidad mínimo para la } \\
\text { energía nuclear. }\end{array}$ & $\begin{array}{l}\text { Adimen } \\
\text { sional }\end{array}$ & 0.6 & 0.9 & 0.3333 \\
\hline $\begin{array}{l}\text { min_energy_intensity_vs_i } \\
\text { ntial }\end{array}$ & $\begin{array}{l}\text { Valor mínimo alcanzable por la intensidad } \\
\text { energética de todos los sectores } \\
\text { económicos en relación con su valor en el } \\
\text { año } 2009 \text {. }\end{array}$ & $\begin{array}{l}\text { Adimen } \\
\text { sional }\end{array}$ & 0.3 & 0.45 & 0.15 \\
\hline $\begin{array}{l}\text { min_energy_intensity_vs_i } \\
\text { ntial_h }\end{array}$ & $\begin{array}{l}\text { Valor mínimo alcanzable por la intensidad } \\
\text { energética de los hogares en relación con } \\
\text { su valor en el año } 2009 .\end{array}$ & $\begin{array}{l}\text { Adimen } \\
\text { sional }\end{array}$ & 0.3 & 0.45 & 0.15 \\
\hline min_lifetime_ev_batteries & $\begin{array}{l}\text { Vida útil mínima de las baterías de los } \\
\text { vehículos eléctricos. }\end{array}$ & Años & 5.0 & 10.0 & 2.5 \\
\hline $\begin{array}{l}\text { share_energy_requiremen } \\
\text { ts_for_decom_ev_batterie } \\
\text { s }\end{array}$ & $\begin{array}{l}\text { Parte de la energía necesaria para el } \\
\text { desmantelamiento de las baterías de los } \\
\text { vehículos eléctricos. }\end{array}$ & $\begin{array}{l}\text { Adimen } \\
\text { sional }\end{array}$ & 0.1 & 0.2 & 0.05 \\
\hline $\begin{array}{l}\text { share_gasxdivxxcoalxplus } \\
\text { xgasx_for_heat_plants }\end{array}$ & $\begin{array}{l}\text { Parte de la generación de calor cubierta } \\
\text { por gas natural en relación al cubierto por } \\
\text { gas natural y carbón conjuntamente. }\end{array}$ & $\begin{array}{l}\text { Adimen } \\
\text { sional }\end{array}$ & 0.72 & 1.0 & 0.0 \\
\hline
\end{tabular}




\begin{tabular}{|c|c|c|c|c|c|}
\hline $\begin{array}{l}\text { Nombre del parámetro } \\
\text { exógeno en Pymedeas }\end{array}$ & Descripción & Unidad & $\begin{array}{l}\text { Valor } \\
\text { nom. }\end{array}$ & $\begin{array}{l}\text { Valor } \\
\max .\end{array}$ & $\begin{array}{l}\text { Valor } \\
\text { min. }\end{array}$ \\
\hline $\begin{array}{l}\text { share_max_of_change_vs_ } \\
\text { historical_mean_h }\end{array}$ & $\begin{array}{l}\text { Tasa máxima de cambio de las } \\
\text { intensidades energéticas de los hogares } \\
\text { (por fuente final) con respecto a las } \\
\text { tendencias históricas. }\end{array}$ & $\begin{array}{l}\text { Adimen } \\
\text { sional }\end{array}$ & 0.5 & 0.75 & 0.25 \\
\hline $\begin{array}{l}\text { share_max_of_change_vs_ } \\
\text { historical_mean_rate }\end{array}$ & $\begin{array}{l}\text { Tasa máxima de cambio de las } \\
\text { intensidades energéticas de todos los } \\
\text { sectores económicos (por fuente final) con } \\
\text { respecto a las tendencias históricas. }\end{array}$ & $\begin{array}{l}\text { Adimen } \\
\text { sional }\end{array}$ & 0.5 & 0.75 & 0.25 \\
\hline $\begin{array}{l}\text { share_res_elec_generation } \\
\text { _curtailedxandxstored }\end{array}$ & $\begin{array}{l}\text { Parte de la generación de electricidad } \\
\text { cubierta por tecnologías de energía } \\
\text { renovable restringida o almacenada. }\end{array}$ & $\begin{array}{l}\text { Adimen } \\
\text { sional }\end{array}$ & 0.2 & 0.5 & 0.0 \\
\hline $\begin{array}{l}\text { threshold_remaining_pote } \\
\text { ntial_new_capacity }\end{array}$ & $\begin{array}{l}\text { Valor del potencial restante de cada } \\
\text { tecnología renovable para la generación de } \\
\text { electricidad por debajo del cual la } \\
\text { planificación de nueva capacidad no es } \\
\text { económicamente viable }\end{array}$ & $\begin{array}{l}\text { Adimen } \\
\text { sional }\end{array}$ & 0.5 & 0.9 & 0.1 \\
\hline $\begin{array}{l}\text { share_energy_requiremen } \\
\text { ts_for_decom_res_elec }\end{array}$ & $\begin{array}{l}\text { Necesidades energéticas para el } \\
\text { desmantelamiento de las plantas de } \\
\text { generación de electricidad renovable en } \\
\text { función de las necesidades energéticas } \\
\text { para la construcción. }\end{array}$ & $\begin{array}{l}\text { Adimen } \\
\text { sional }\end{array}$ & 0.1 & 0.2 & 0.05 \\
\hline
\end{tabular}

Además, en este análisis de sensibilidad se ha evaluado el impacto de dos parámetros endógenos del modelo (ver Tabla 5.7) multiplicando/dividiendo su valor por una constante.

Tabla 5.7: Nombres de los parámetros endógenos en pymedeas_eu 0.3.0 utilizados para el análisis de sensibilidad junto con su descripción, sus unidades, los índices afectados y sus valores mínimos y máximos.

Fuente: (Samsó et al., 2020).

\begin{tabular}{|c|c|c|c|c|c|}
\hline $\begin{array}{l}\text { Nombre del parámetro } \\
\text { endógeno en Pymedeas }\end{array}$ & Descripción & Unidad & $\begin{array}{l}\text { Subíndices } \\
\text { afectados }\end{array}$ & Mínimo & Máximo \\
\hline variation_nonxdashxenergy_use & $\begin{array}{l}\text { Variación anual de los } \\
\text { combustibles para usos } \\
\text { no energéticos. }\end{array}$ & EJ & $\begin{array}{l}\text { líquidos, } \\
\text { gases, sólidos. }\end{array}$ & $\mathrm{x} 0.5$ & $\mathrm{x} 2.0$ \\
\hline energy_per_x_t & $\begin{array}{l}\text { Energía por actividad } \\
\text { económica del sector del } \\
\text { transporte terrestre. }\end{array}$ & $\begin{array}{l}\mathrm{EJ} / 10^{12} \\
1995 \\
\mathrm{US} \$\end{array}$ & $\begin{array}{lr}\text { Todos } & \text { los } \\
\text { vehículos } & \text { de } \\
\text { transporte } & \\
\text { terrestre } & \end{array}$ & $\mathrm{x} 0.5$ & $\mathrm{x} 2.0$ \\
\hline
\end{tabular}

El análisis de sensibilidad se ha realizado sobre la versión "pymedeas_eu 0.3.0" que es una traducción del modelo MEDEAS-EU v1.2 a lenguaje de programación Python que permite realizar mejor este tipo de análisis. Se han realizado 43 simulaciones modificando únicamente un parámetro en cada simulación: 21 con los valores superiores, 21 con los valores inferiores y 1 con los valores nominales. Para analizar los resultados de las simulaciones se han utilizado tanto medidas cualitativas como cuantitativas:

- "Spider plot": trazar los valores de todas las variables de salida al final del tiempo de simulación frente al porcentaje de cambio de cada parámetro con respecto a su valor nominal. 
- Raíz del error cuadrático medio (RMSD) entre las curvas obtenidas para cada salida con los valores máximo y mínimo de los parámetros utilizados.

- Distancia euclidiana entre todas las salidas obtenidas con los valores modificados y nominal de cada parámetro utilizado.

Samsó et al., describe de manera detallada en su trabajo las ecuaciones con las que se obtienen las medidas cuantitativas (Samsó et al., 2020).

La lista de variables de salida sobre las que se evalúa esta prueba se han elegido a partir de una comparación con las salidas más utilizadas en otros modelos de la literatura y se muestran en la Tabla 5.8.

Tabla 5.8: Lista de salidas del modelo MEDEAS-EU que se han utilizado en la prueba de análisis de sensibilidad.

Fuente: (Samsó et al., 2020).

\begin{tabular}{|c|c|c|}
\hline $\begin{array}{l}\text { Nombre del parámetro de } \\
\text { salida }\end{array}$ & Descripción & Unidades \\
\hline eroist_system & EROI estándar del sistema & Adimensional \\
\hline gdp & PIB & $10^{12} 1995$ US\$ \\
\hline gdppc & PIB per cápita & \$/persona \\
\hline $\begin{array}{l}\text { real_fe_consumption_by_fuel[ } \\
\text { electricity] }\end{array}$ & $\begin{array}{l}\text { Consumo final de energía por combustible después de } \\
\text { confrontar con la disponibilidad de energía (electricidad) }\end{array}$ & EJ \\
\hline $\begin{array}{l}\text { real_fe_consumption_by_fuel[ } \\
\text { gases] }\end{array}$ & $\begin{array}{l}\text { Consumo final de energía por combustible después de } \\
\text { confrontar con la disponibilidad de energía (gases) }\end{array}$ & EJ \\
\hline $\begin{array}{l}\text { real_fe_consumption_by_fuel[ } \\
\text { heat] }\end{array}$ & $\begin{array}{l}\text { Consumo final de energía por combustible después de } \\
\text { confrontar con la disponibilidad de energía (calor) }\end{array}$ & EJ \\
\hline $\begin{array}{l}\text { real_fe_consumption_by_fuel[ } \\
\text { liquids] }\end{array}$ & $\begin{array}{l}\text { Consumo final de energía por combustible después de } \\
\text { confrontar con la disponibilidad de energía (líquidos) }\end{array}$ & EJ \\
\hline $\begin{array}{l}\text { real_fe_consumption_by_fuel[ } \\
\text { solids] }\end{array}$ & $\begin{array}{l}\text { Consumo final de energía por combustible después de } \\
\text { confrontar con la disponibilidad de energía (sólidos) }\end{array}$ & EJ \\
\hline real_tfec & $\begin{array}{l}\text { Consumo total de energía final (sin incluir los usos no } \\
\text { energéticos) }\end{array}$ & EJ \\
\hline $\begin{array}{l}\text { remaining_potential_tot_res_ } \\
\text { elec }\end{array}$ & $\begin{array}{l}\text { Potencial restante de energía para producción de electricidad } \\
\text { en función del potencial total. }\end{array}$ & Adimensional \\
\hline $\begin{array}{l}\text { share_res_electricity_generati } \\
\text { on }\end{array}$ & $\begin{array}{l}\text { Parte de la generación de electricidad cubierta por energías } \\
\text { renovables }\end{array}$ & Adimensional \\
\hline total_co2_emissions_gtco2 & Emisiones totales de $\mathrm{CO}_{2}$ & $\mathrm{Gt} \mathrm{CO}_{2} /$ Year \\
\hline total_fe_elec_generation_twh & Generación total de energía eléctrica & TWh \\
\hline $\begin{array}{l}\text { total_land_requirements_rene } \\
\text { w_mha }\end{array}$ & Requerimientos de tierra para las energías renovables & MHa \\
\hline tpes_intensity_ej_tdollar & Intensidad energética primaria total & $\begin{array}{ll}\mathrm{EJ} / 10^{12} & 1995 \\
\mathrm{US} \$ & \end{array}$ \\
\hline
\end{tabular}

Las gráficas "spider plots" se utilizan para evaluar cualitativamente el impacto de las modificaciones en los parámetros de la Tabla 5.6 y la Tabla 5.7 en las variables de salida de la 
Tabla 5.8 en el año 2050. En la Figura 5.17 se muestra como se ven afectadas seis de las salidas por las modificaciones en los parámetros de entrada.
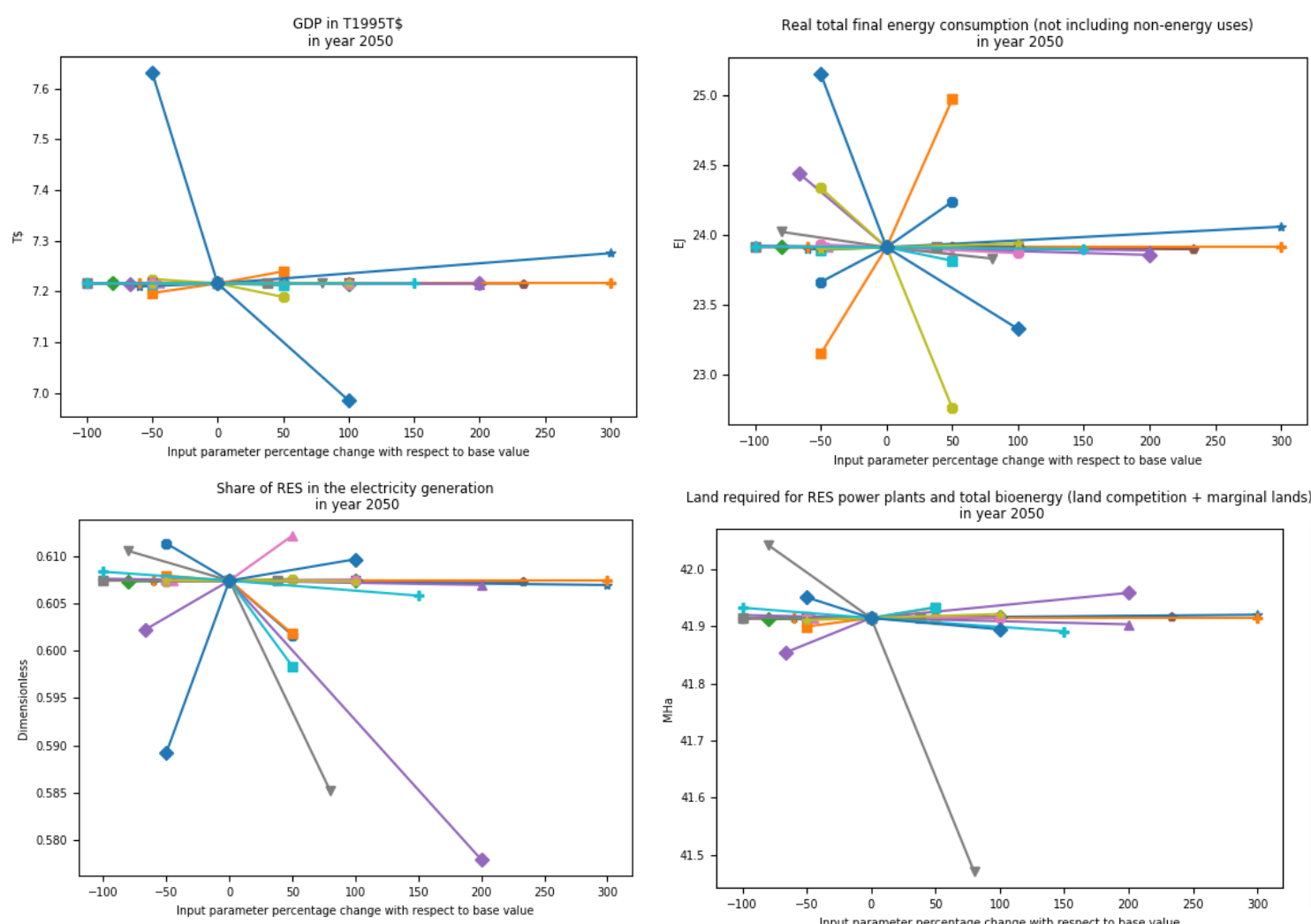

Land required for RES power plants and total bioenergy (land competition + marginal lands)
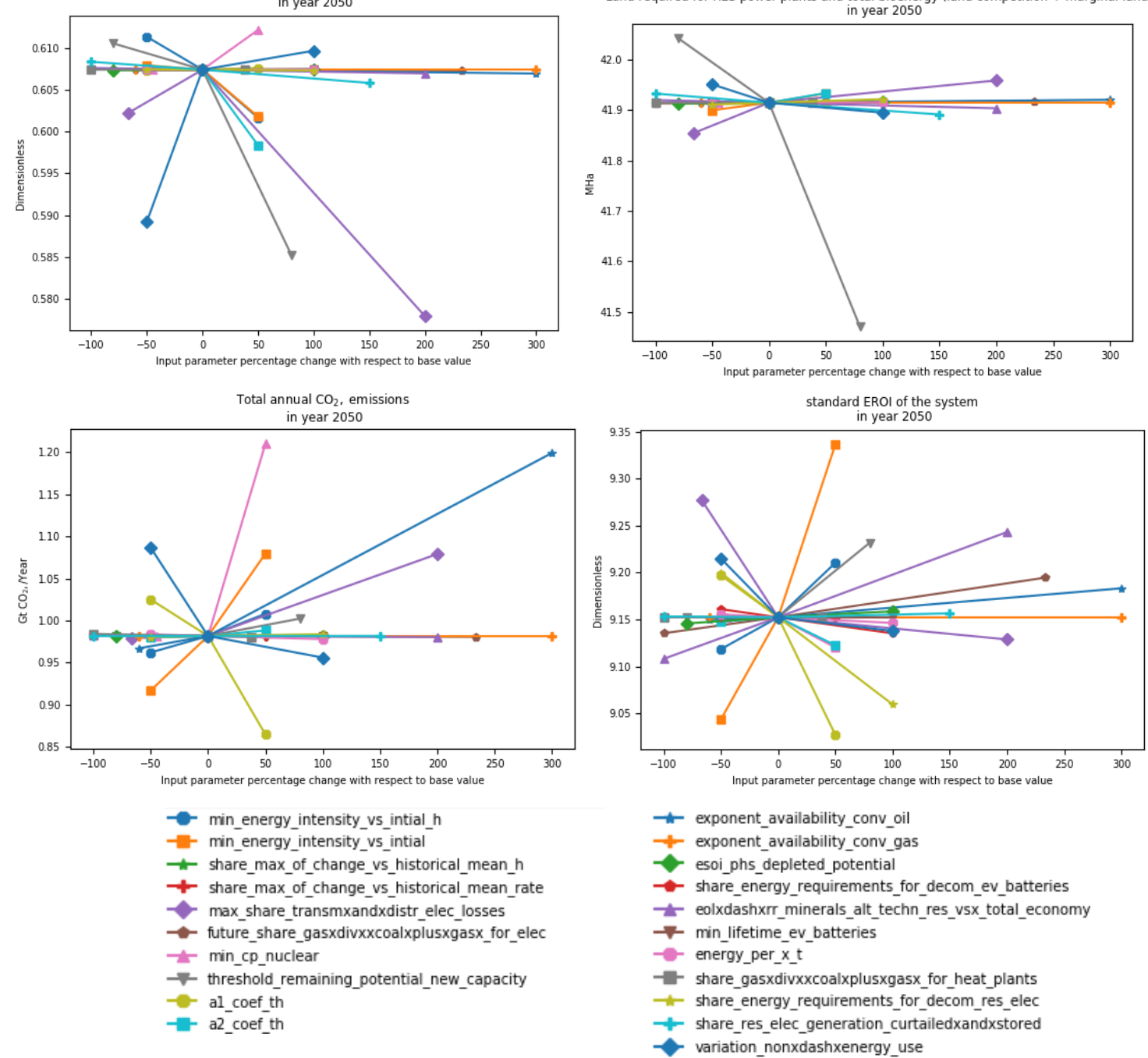

Figura 5.17: "Spider plots" en el año 2050 obtenidas en el análisis de sensibilidad realizado a pymedeas_eu 0.3.0.

Notas: De arriba abajo y de izquierda a derecha, PIB, consumo de energía final, parte de la electricidad producida por energías renovables, requerimientos de tierra para energías renovables, emisiones totales de $\mathrm{CO}_{2}$ y EROI estándar del sistema.

Fuente: (Samsó et al., 2020). 
Todas las variables de salida se han visto afectadas por la modificación individual de las variables de entradas, aunque en grados muy diferentes. Por lo general, el aumento o disminución del valor del parámetro de entrada no ha dado lugar a aumentos o disminuciones proporcionales en las variables de salida, lo que muestra una relación no lineal entre las entradas y salidas del modelo.

Las modificaciones del parámetro "variation_non-energy_use" afectaron significativamente a todas las salidas del modelo, la variación es particularmente importante en el PIB como se puede ver en la Figura 5.17. Las modificaciones del parámetro "min_energy_intensity" también producen cambios notables en una gran parte de las variables de salida siendo el EROI estándar del sistema y el consumo total de energía las más afectadas.

Otras conclusiones muy claras de este análisis de sensibilidad son: 1) el aumento del parámetro "threshold_remaining_potential_new_capacity" tiene un impacto negativo muy importante en la parte de la electricidad producida por energías renovables y en los requerimientos de tierra para renovable; 2) el parámetro "al_coef_th", relacionado con el consumo de energía del transporte en función de la producción económica, tiene una relación importante con el consumo total de energía final, las emisiones de $\mathrm{CO}_{2}$ y el EROI estándar del sistema; 3) existe una relación importante entre la variable "min_cp_nuclear" y las emisiones de $\mathrm{CO}_{2}$.

El análisis cuantitativo, a través de la raíz de error cuadrático medio y la distancia euclidiana, muestra que los parámetros de entrada "variation_non-energy_use", "min_energy_intensity" y "al_coef_th" son los parámetros que más impactan a las salidas estudiadas, afectando a 15, 8 y 7 de las salidas respectivamente. Estos resultados confirman los resultados cualitativos obtenidos con las gráficas "spider plots". Los resultados obtenidos en el análisis de sensibilidad cuantitativo se pueden revisar en el trabajo de Samsó et al., (Samsó et al., 2020).

Por el contrario, el análisis de sensibilidad nos ha mostrado que parámetros como "min_lifetime_ev_batteries", "share_energy_requirements_for_decom_ev_batteries" o "exponent_availability_conv_gas" tienen muy poca influencia sobre las principales variables de salida del modelo.

Los resultados de esta prueba de análisis de sensibilidad son muy importantes para el desarrollo futuro del modelo, indican cuáles son los parámetros del modelo que tienen una mayor influencia sobre las principales salidas. Gracias a este análisis de sensibilidad, actualmente se está trabajando en distintas alternativas al modelado de las partes que han resultado ser muy sensibles como por ejemplo la relación entre el consumo de energía del transporte con su producción económica.

\section{Análisis de incertidumbre}

La mayoría de los parámetros que componen la estructura interna del modelo presentan incertidumbres que por lo general no han sido muy evaluadas en los IAM (Gambhir et al., 2019; Pastor et al., 2020). Esta es una de las limitaciones señaladas en la revisión de los IAM realizada en el capítulo 2 de esta tesis. Es importante remarcar que no mostrar la incertidumbre en modelos complejos como los IAM implica una bajada en la credibilidad de los mismos. En el modelo MEDEAS-W se ha realizado un análisis para estudiar como la incertidumbre en las entradas del modelo se propaga en sus salidas, este análisis ha sido publicado como material suplementario del artículo metodológico de MEDEAS (Capellán-Pérez et al., 2020).

Se han elegido un total de 75 variables correspondientes a las variables exógenas del modelo. Se han tomado amplios rangos de incertidumbre para estudiar su impacto en los resultados obtenidos para los cuatro casos del trabajo de Capellán-Pérez et al., (ver sección ¡Error! No se encuentra 
el origen de la referencia. de está tesis para información sobre los cuatro casos analizados) (Capellán-Pérez et al., 2020). Como puede verse en la Tabla 5.9 se han tomado por defecto distribuciones uniformes aleatorias de $\pm 20 \%$ con referencia al valor del escenario BAU, mientras que para las entradas que se estima que están más afectadas por la incertidumbre se ha considerado un rango más amplio de $\pm 50 \%$ o incluso x2-/2 (es decir, el doble y la mitad).

Tabla 5.9: Rangos de incertidumbre de las variables de entrada para el análisis de incertidumbre realizado en los 4 cuatro casos de estudio de Capellán-Pérez et al., (Capellán-Pérez et al., 2020).

Notas: (UD): Distribución uniforme aleatoria.

Fuente: (Capellán-Pérez et al., 2020).

\begin{tabular}{|c|c|}
\hline Entradas e hipótesis de los escenarios & $\begin{array}{l}\text { Rangos de incertidumbre de las entradas para el análisis de } \\
\text { incertidumbre (en referencia con los valores del escenario } \\
\text { BAU) }\end{array}$ \\
\hline Población (2015-2100) & $\pm 20 \%$ del crecimiento de la población en SSP2 \\
\hline Crecimiento del PIB esperado (2015-2100) & $\begin{array}{l} \pm 20 \% \text { (UD) (el crecimiento medio entre } 2015-2100 \text { en SSP2 } \\
\text { corresponde a } 2.5 \% / \text { ) }\end{array}$ \\
\hline $\begin{array}{l}\text { Objetivo de participación del trabajo en el } \\
\text { PIB }\end{array}$ & $\begin{array}{l} \pm 20 \% \text { (UD) respecto al objetivo establecido en } 2050 \text { (en el } \\
\text { periodo histórico ha habido una variación de un } \pm 5 \% \text { ) }\end{array}$ \\
\hline \multicolumn{2}{|l|}{ Intensidades energéticas finales } \\
\hline $\begin{array}{l}\text { Intensidad energética final mínima respecto } \\
\text { inicial }\end{array}$ & $/ 2 ;+50 \%(\mathrm{UD})$ \\
\hline $\begin{array}{l}\text { \% de cambio sobre la variación histórica } \\
\text { máxima de las intensidades energéticas } \\
\text { finales. }\end{array}$ & $\pm 50 \%$ (UD) \\
\hline Sensibilidad a la escasez & $/ 2 ;+50 \%$ (UD) \\
\hline Factor de olvido de la escasez energética & $12 ;+50 \%$ (UD) \\
\hline \multicolumn{2}{|c|}{$\begin{array}{l}\text { Transporte terrestre y de los hogares } \\
\pm 50 \% \text { (UD) para cada porcentaje objetivo de cada vehículo } \\
\text { Porcentaje objetivo de dependencia del petróleo para cada categoría en } 2100 \text { : }\end{array}$} \\
\hline Vehículos de cuatro ruedas & $\begin{array}{l}\text { 1\%-67\% empezando en 2020-2050 (UD) con el año objetivo en } \\
2051-2100 \text { (UD) }\end{array}$ \\
\hline Vehículos de dos ruedas & $\begin{array}{l}\text { 0\%-50\% empezando en 2020-2050 (UD) con el año objetivo en } \\
2051-2100 \text { (UD) }\end{array}$ \\
\hline Vehículos pesados & $\begin{array}{l}\text { 61\%-87\% empezando en 2020-2050 (UD) con el año objetivo en } \\
2051-2100 \text { (UD) }\end{array}$ \\
\hline Buses & $\begin{array}{l}31 \%-77 \% \text { empezando en 2020-2050 (UD) con el año objetivo en } \\
2051-2100 \text { (UD) }\end{array}$ \\
\hline $\begin{array}{l}\text { Tasa de reciclado de minerales } \\
\text { (19 minerales) }\end{array}$ & $\begin{array}{l}\text { Mejora del 0\%-5\% (UD) anual de la tasa de reciclado entre los } \\
\text { años } 2020 \text { y } 2050 \text { (UD) }\end{array}$ \\
\hline Capacidad nuclear & $\begin{array}{l}\text { Variación de la capacidad nuclear de 0-5\%/año (UD) entre los } \\
\text { años } 2020 \text { y } 2050 \text { (UD) }\end{array}$ \\
\hline \multicolumn{2}{|c|}{ Curvas de agotamiento de las energías no renovables } \\
\hline Petróleo & 0-25\% (UD) para la URR y el nivel de extracción máxima \\
\hline Gas Natural & 0-25\% (UD) para la URR y el nivel de extracción máxima \\
\hline Carbón & 0-25\% (UD) para la URR y el nivel de extracción máxima \\
\hline Uranio & 0-25\% (UD) para la URR y el nivel de extracción máxima \\
\hline $\begin{array}{l}\text { Emisiones de GEI de otros gases que no son } \\
\mathrm{CO}_{2} \text { and } \mathrm{CH}_{4}\end{array}$ & $\begin{array}{l}50 \% \text { probabilidad RCP6.0 } \\
50 \% \text { probabilidad RCP } 8.5\end{array}$ \\
\hline
\end{tabular}




\begin{tabular}{|c|c|c|}
\hline Entradas e hipótesis de los escenarios & \multicolumn{2}{|c|}{$\begin{array}{l}\text { Rangos de incertidumbre de las entradas para el análisis de } \\
\text { incertidumbre (en referencia con los valores del escenario } \\
\text { BAU) }\end{array}$} \\
\hline Impactos del cambio climático & \multicolumn{2}{|c|}{$\begin{array}{l}50 \% \text { prob. de la función de daño calibrada a }+1.75^{\circ} \mathrm{C} \text { con unas } \\
\text { pérdidas del PIBpc del } 1 \% \\
50 \% \text { prob. de la función de daño calibrada a }+1.75^{\circ} \mathrm{C} \text { con unas } \\
\text { pérdidas del PIBpc del } 3 \%\end{array}$} \\
\hline \multicolumn{3}{|l|}{ Energías renovables } \\
\hline & $\begin{array}{l}\text { Capacidad crecimiento anual } \\
(2015-2100)\end{array}$ & Potencial técno-sostenible \\
\hline $\begin{array}{l}\text { Todas las tecnologías para generación de } \\
\text { electricidad }\end{array}$ & $\pm 20 \%$ (UD) & /2-x2 (UD) \\
\hline $\begin{array}{l}\text { Todas las tecnologías para generación de } \\
\text { calor }\end{array}$ & $\pm 20 \%$ (UD) & /2-x2 (UD) \\
\hline Bioenergía convencional & - & /2-x2 (UD) \\
\hline $\begin{array}{l}\text { Bioenergía de campos de cultivo de } 2^{\mathrm{a}} \\
\text { generación }\end{array}$ & $\pm 20 \%$ (UD) & \multirow{2}{*}{ /2-x2 (UD) } \\
\hline $\begin{array}{l}\text { Bioenergía de campos de cultivo de } 3^{\mathrm{a}} \\
\text { generación }\end{array}$ & $\begin{array}{l} \pm 20 \% \text { (UD) empezando en } 2025 \text { - } \\
2050 \text { (UD) }\end{array}$ & \\
\hline Bioenergía de residuos & $\begin{array}{l} \pm 20 \% \text { (UD) empezando en } 2025- \\
2050 \text { (UD) }\end{array}$ & /2-x2 (UD) \\
\hline Bioenergía de tierras marginales & $\begin{array}{l} \pm 20 \% \text { (UD) empezando en } 2025- \\
2050 \text { (UD) }\end{array}$ & $\pm 20 \%$ (UD) \\
\hline
\end{tabular}

Para realizar el análisis de incertidumbre se ha utilizado, como en otras pruebas en el proceso de validación, la herramienta de Vensim DSS para opciones de sensibilidad, se han realizado 1000 simulaciones de Monte Carlo al modelo MEDEAS-W v1.3.33. Los resultados de esta prueba se han analizado cualitativamente utilizando gráficas que muestran los intervalos de confianza en torno a la media de la distribución para cada variable de salida. A continuación se muestran los resultados de algunas variables: consumo total de energía final per cápita (Figura 5.18), parte de la energía primaria total cubierta por energías renovables (Figura 5.19), producto interior bruto per cápita (Figura 5.20), emisiones totales de gases de efecto invernadero (Figura 5.21), aumento en la temperatura global (Figura 5.22) e intensidad de $\mathrm{CO}_{2}$ eq en la energía final (Figura 5.23). La línea roja muestra los cuatro casos reportados en el trabajo de Capellán-Pérez et al., (CapellánPérez et al., 2020). 


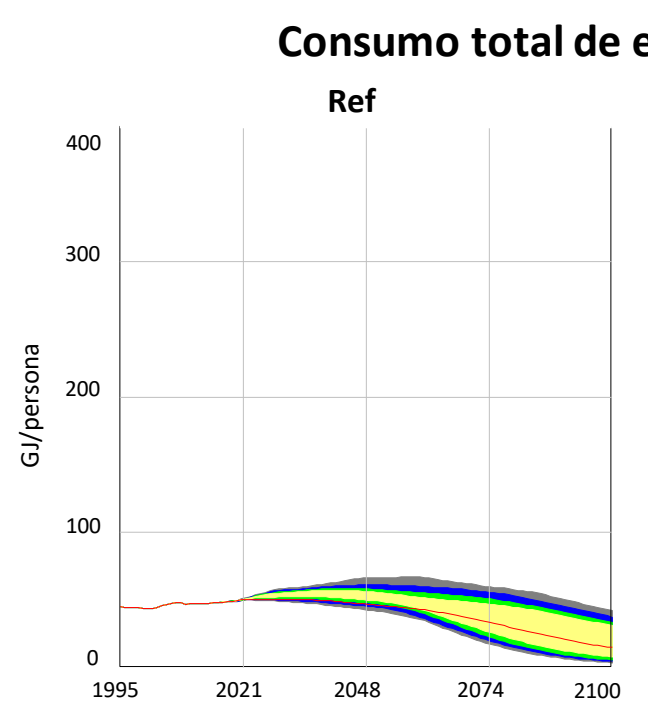

energía final per cápita
Ref noER
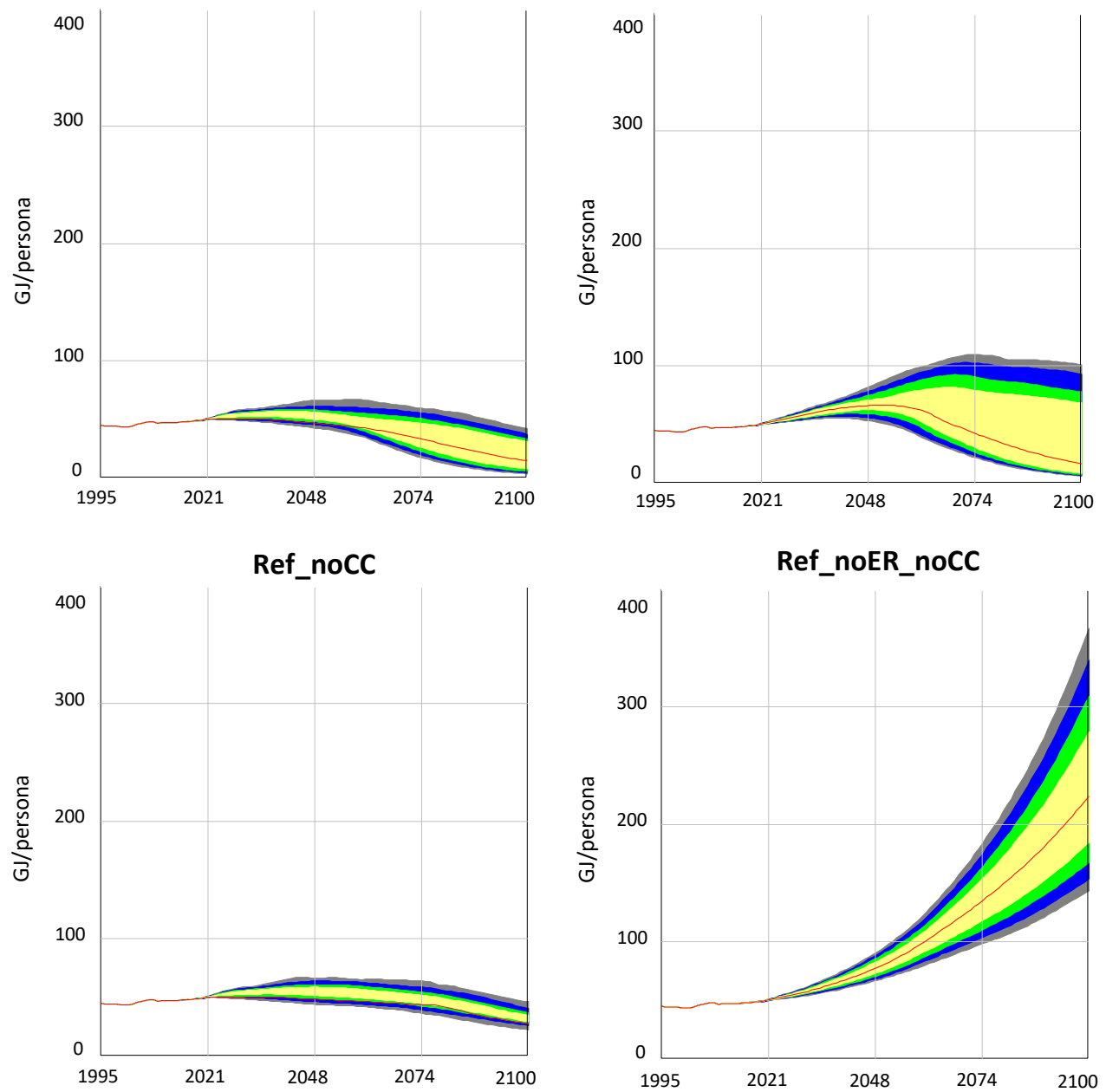

Intervalos de

confianza

$50 \%$
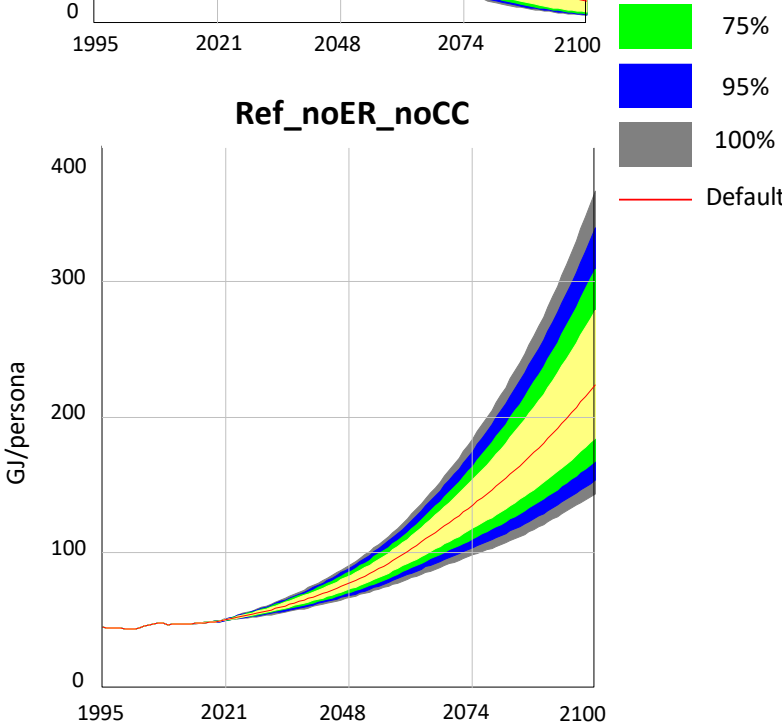

Figura 5.18: Distribución de probabilidades del consumo total de energía final per cápita en GJ por persona en MEDEAS-W v1.3.33, para los cuatro casos considerados en Capellán-Pérez et al (Capellán-Pérez et al., 2020).

Notas: Las áreas coloreadas representan los intervalos de confianza. 


\section{Energías renovables vs energía primaria total}
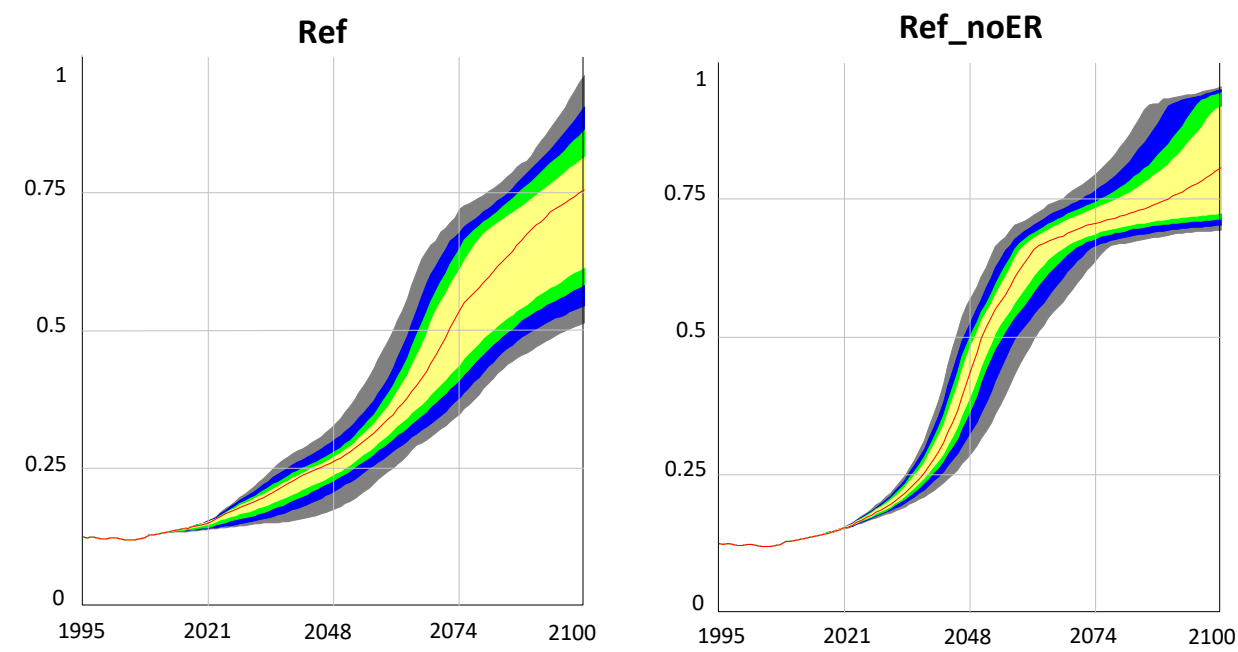

Intervalos de

confianza
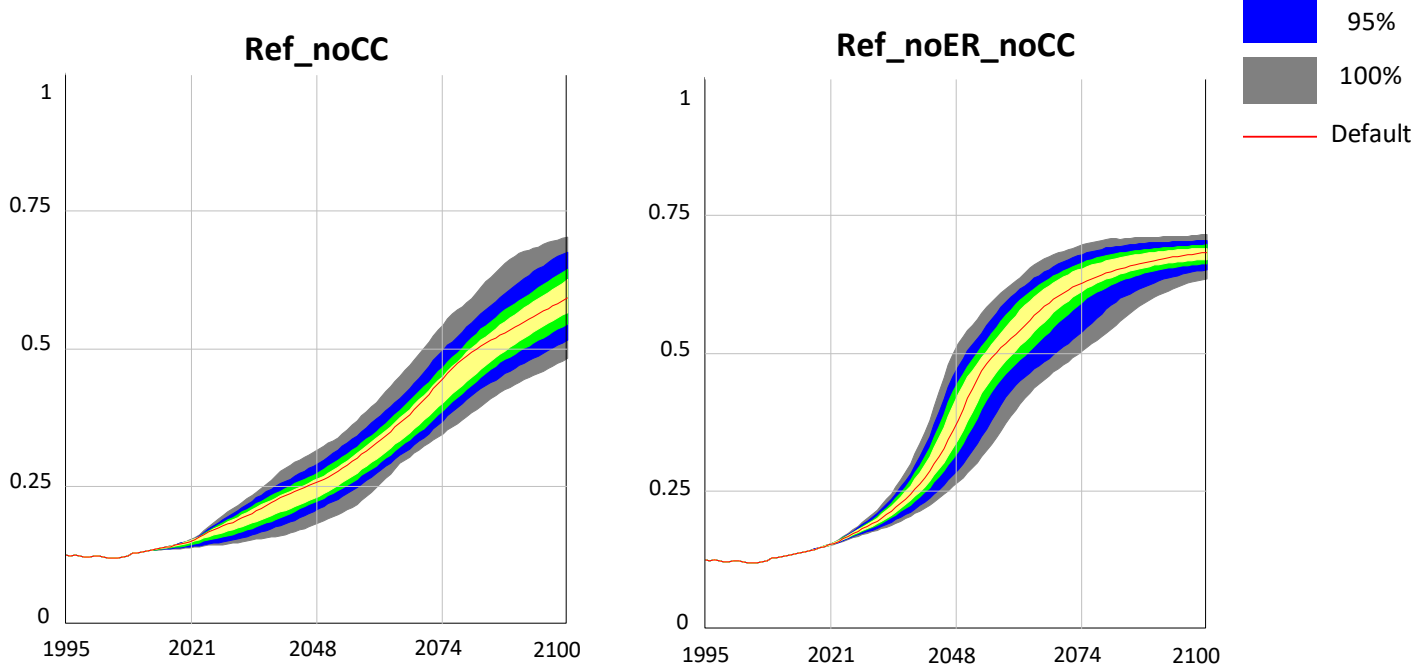

Figura 5.19: Distribución de probabilidades de la parte de la energía primaria total cubierta por energías renovables en MEDEAS-W v1.3.33 para los cuatro casos considerados en CapellánPérez et al (Capellán-Pérez et al., 2020).

Notas: Las áreas coloreadas representan los intervalos de confianza. 


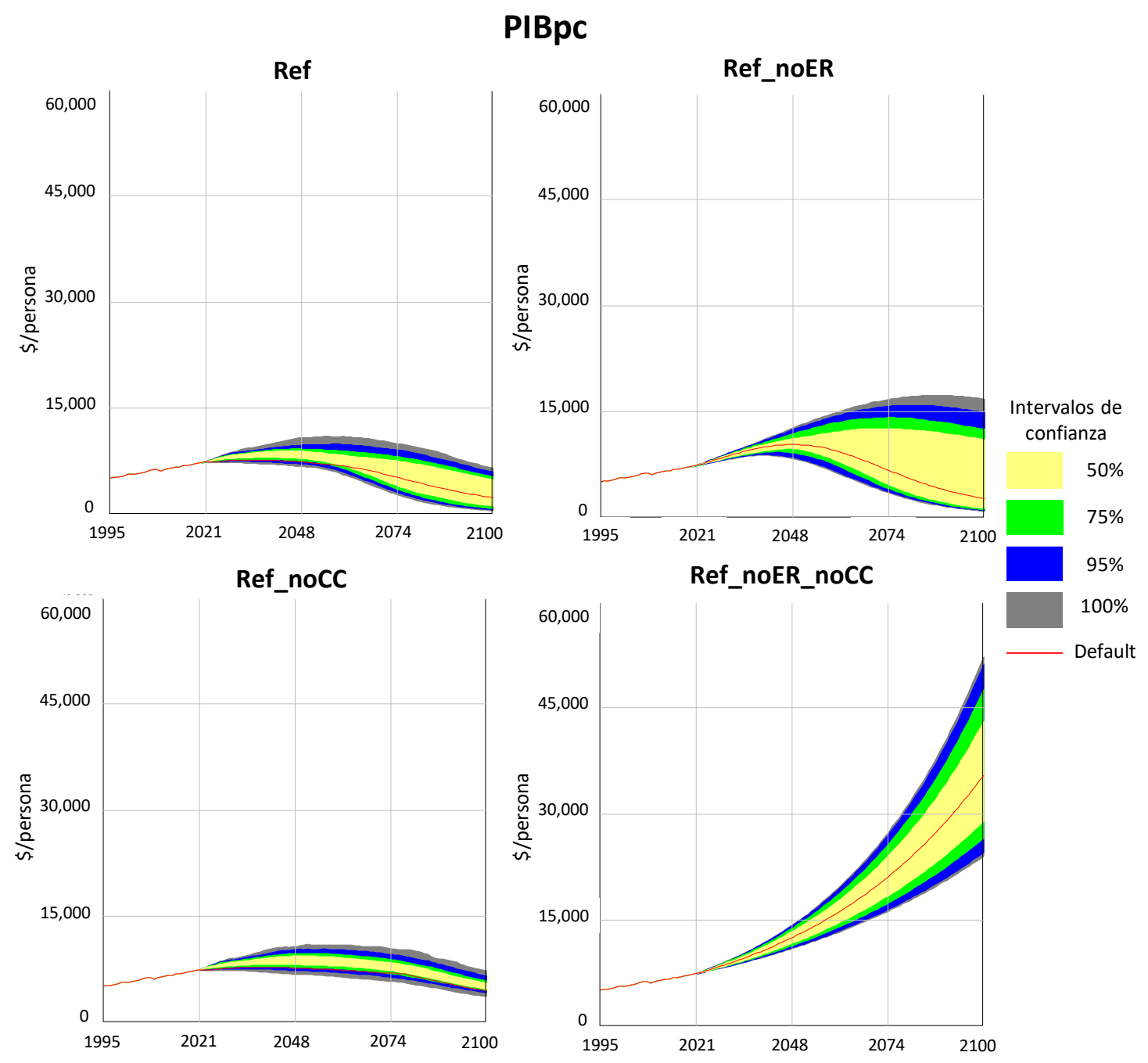

Figura 5.20: Distribución de probabilidades del producto interior bruto per cápita (PIBpc) en dólares de EEUU del año 1995 en MEDEAS-W v1.3.33, para los cuatro casos considerados en Capellán-Pérez et al (Capellán-Pérez et al., 2020).

Notas: Las áreas coloreadas representan los intervalos de confianza. 


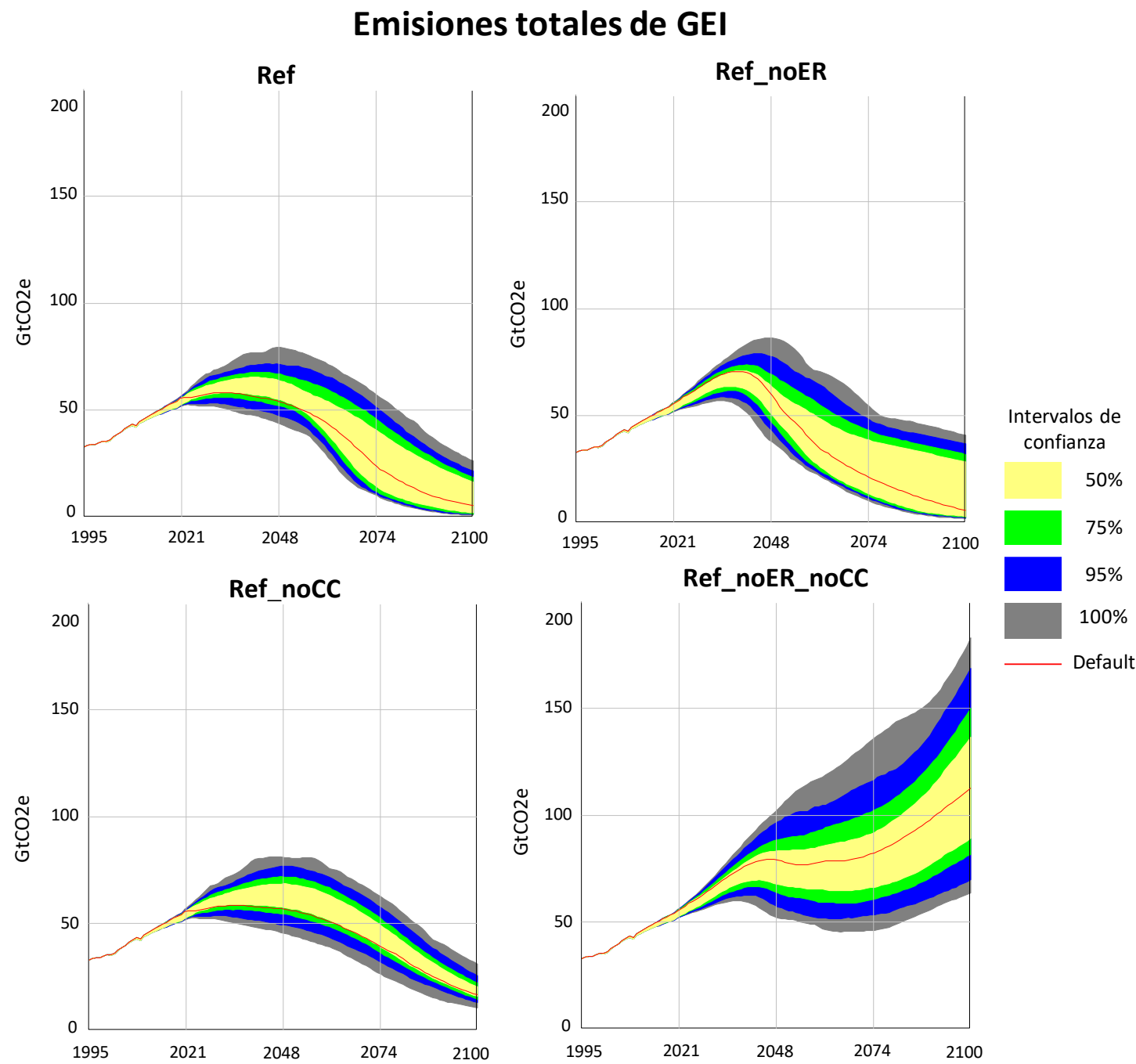

Figura 5.21: Distribución de probabilidades de las emisiones totales de gases de efecto invernadero en gigatoneladas de $\mathrm{CO}_{2}$ equivalente en MEDEAS-W v1.3.33, para los cuatro casos considerados en Capellán-Pérez et al (Capellán-Pérez et al., 2020).

Notas: Las áreas coloreadas representan los intervalos de confianza. 


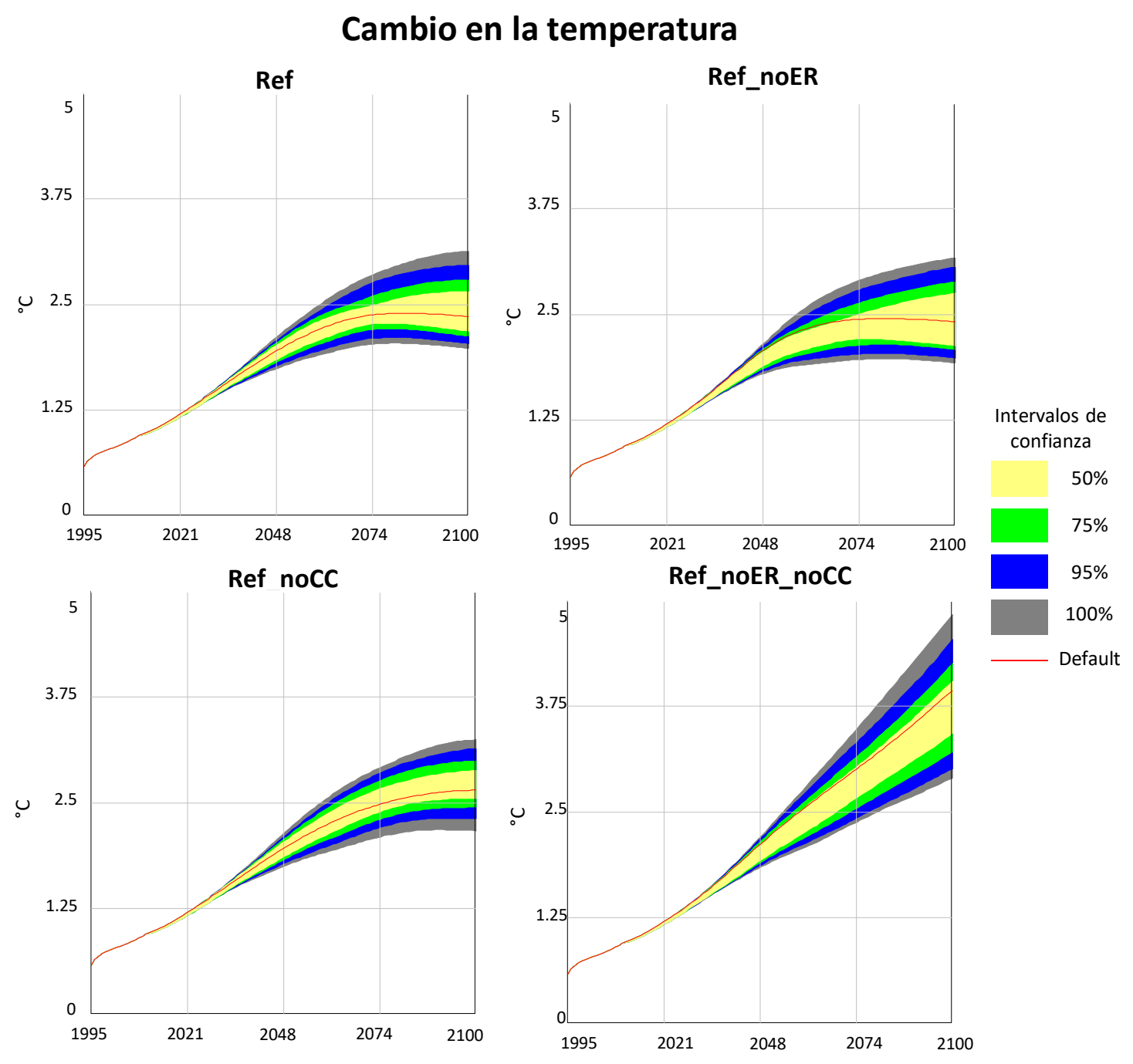

Figura 5.22: Distribución de probabilidades del aumento en la temperatura global en ${ }^{\circ} \mathrm{C}$ en MEDEAS-W v1.3.33, para los cuatro casos considerados en Capellán-Pérez et al (CapellánPérez et al., 2020).

Notas: Las áreas coloreadas representan los intervalos de confianza. 


\section{Intensidad de $\mathrm{CO}_{2}$ eq en la energía final}
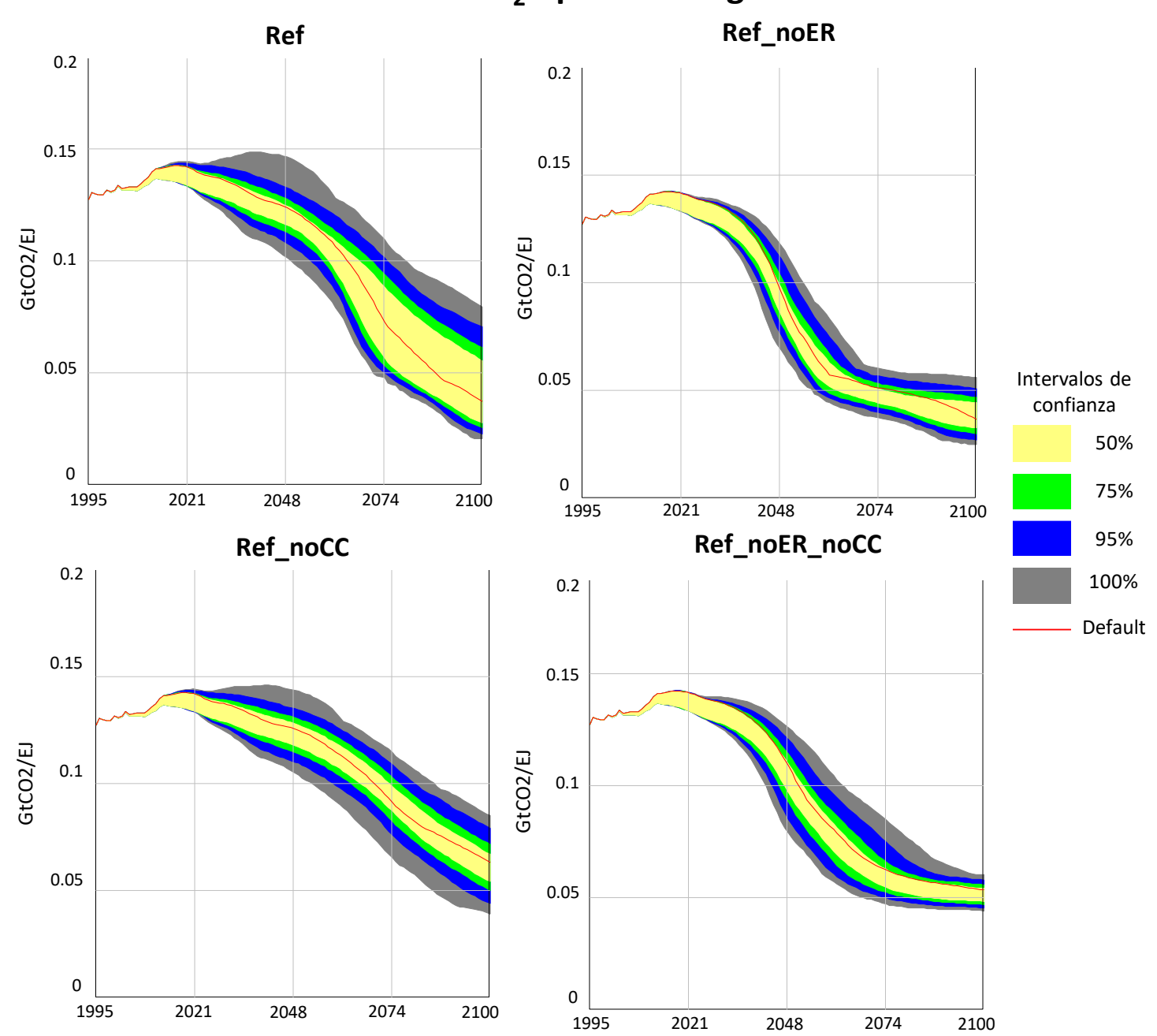

Figura 5.23: Distribución de probabilidades de la intensidad de $\mathrm{CO}_{2}$ equivalente en la energía final en gigatoneladas de $\mathrm{CO}_{2}$ equivalente partido EJ en MEDEAS-W v1.3.33, para los cuatro casos considerados en Capellán-Pérez et al (Capellán-Pérez et al., 2020).

Notas: Las áreas coloreadas representan los intervalos de confianza.

La incertidumbre en los valores de entrada se propaga en el modelo generando una incertidumbre en las variables de salida en el año 2100. Por lo general, cabe señalar que se obtiene un buen nivel de estabilidad en los resultados para los cuatro casos estudiados pese a los amplios rangos de incertidumbre considerados en las variables de entrada. La simulación del caso Ref_noER_noCC donde no hay limitaciones climáticas ni energéticas genera unos rangos de incertidumbre mayores que en el resto de simulaciones en algunas de sus salidas: el consumo total de energía final per cápita (150-370 GJ/persona), el PIB per cápita (25000-50000 US1995\$/persona), las emisiones totales de GEI (65-185 $\left.\mathrm{GtCO}_{2} \mathrm{e}\right)$ o el cambio en la temperatura $\left(3-5^{\circ} \mathrm{C}\right)$. Estos amplios rangos son debidos a que, en este caso, las variables del modelo crecen ilimitadamente al no existir restricciones ni climáticas ni energéticas y la incertidumbre en los valores de entrada se propaga sin limitaciones a las variables de salida. En el resto de casos planteados: Ref, Ref_noER y Ref_noCC, el rango de incertidumbre en las salidas es menor. En estas simulaciones las limitaciones del modelo, ya sean energéticas o climáticas, aparecen antes o después durante el siglo XXI e impiden un crecimiento ilimitado tanto del valor por defecto como de la incertidumbre. También es importante señalar que hay una cierta asimetría en los resultados 
respecto al valor por defecto de cada caso (línea roja) debido, sobre todo, a que los rangos de incertidumbre para la disponibilidad de recursos fósiles son asimétricos.

Sterman señala que lo importante de este análisis es comprobar si las conclusiones cambian de manera importante cuando las variables de entrada varían con rangos factibles de incertidumbre (Sterman, 2000). En nuestro caso, las conclusiones obtenidas en la simulación con los valores por defecto son muy parecidas después de aplicar unos amplios rangos de incertidumbre en las entradas. Los escenarios en los que los límites del sistema impedían tener un crecimiento económico ilimitado muestran ese mismo comportamiento una vez considerada la incertidumbre de las entradas, el pico en el producto interior bruto per cápita o el consumo de energía per cápita se puede adelantar o retrasar, pero en cualquier caso acaba llegando antes del final de la simulación (ver Figura 5.18 y Figura 5.20). Aunque las conclusiones sean parecidas es importante acompañar los resultados siempre de sus rangos de incertidumbre, como se ha hecho en el estudio de Capellán-Pérez et al., para que cualquier usuario pueda tener una visión general de la incertidumbre existente y no entender los resultados como exactos (Capellán-Pérez et al., 2020).

También hay que tener en cuenta que en el modelo hay muchas más fuentes de incertidumbre que los parámetros de entrada, existe también una incertidumbre estructural que hay considerar a la hora de valorar los resultados (Pastor et al., 2020).

\subsubsection{Pruebas de patrones de comportamiento}

Una vez se ha generado suficiente confianza en la validez de la estructura del modelo, mediante pruebas directas o indirectas, se pueden aplicar al modelo pruebas diseñadas para medir la precisión con la que el modelo puede reproducir los principales patrones de comportamiento del sistema real (Barlas, 1996). Es importante remarcar que el objetivo es comparar los patrones de comportamiento (periodos, frecuencias, tendencias, amplitudes) más que la predicción de puntos exactos. Ejemplos de pruebas de patrones de comportamiento son la evaluación de los patrones de comportamiento, la prueba de comportamiento anómalo del sistema, la prueba de comportamiento de distintos miembros de la misma familia o la prueba de comportamiento sorpresa.

\section{Evaluación de los patrones de comportamiento}

Las herramientas más comunes para evaluar la capacidad de un modelo de reproducir el comportamiento de un sistema son los métodos estadísticos estándar como el coeficiente de determinación $\mathrm{R}^{2}$, la media o la varianza. Sin embargo, en la validación de la dinámica de sistemas se utilizan muy poco las pruebas estadísticas estándar (Barlas, 1996). Los problemas que plantea la utilización de estas pruebas son muchos. Por ejemplo, la mayoría de las pruebas estadísticas suponen que los datos sean independientes (no autocorrelacionados), no relacionados transversalmente y normalmente distribuidos. Sin embargo, los datos generados por los modelos de dinámica de sistemas son, por su propia naturaleza, autocorrelacionados y relacionados transversalmente, por lo que no se pueden utilizar con éxito las pruebas estadísticas. Además, en los modelos de dinámica de sistemas no suele haber una única variable de salida en la que se puedan centrar las pruebas de patrones de comportamiento, normalmente hay muchas variables importantes para analizar.

La alternativa es realizar pruebas cualitativas de evaluación del comportamiento para analizar los patrones obtenidos en el modelo. En MEDEAS-W el análisis de los patrones de comportamiento 
será cualitativo, sin entrar en cuestiones estadísticas, y es necesario hacerlo tanto de los datos históricos del modelo como de sus predicciones futuras.

Un ejemplo de prueba cualitativa de evaluación de los patrones de comportamiento es la prueba de generación de síntomas. Esta prueba examina si el modelo recrea o no los problemas que han motivado la construcción del modelo. Por lo general, un modelo se desarrolla para solucionar un problema, por lo que si el modelo no es capaz de generar dicho problema no podrá alterar las causas que lo provocan. En el caso de MEDEAS-W, los síntomas que debe mostrar el modelo son aquellos que muestren un deterioro ambiental del planeta como, por ejemplo, el aumento de la temperatura global por encima de $\operatorname{los} 2^{\circ} \mathrm{C}$, la subida del nivel del mar, el agotamiento de una parte de los minerales utilizados actualmente o importantes cambios en el uso del suelo debido, por ejemplo, a la deforestación o al uso de biocombustibles. El modelo MEDEAS-W v2.0 es capaz de recrear esos comportamientos en el escenario BAU como se observa en la Figura 5.24. Es necesario destacar que algunos de estos síntomas son frutos de varias realimentaciones, como es el caso del aumento en la temperatura, y otros son únicamente variables de salida, como la escasez de minerales, cuya realimentación sería necesaria para conocer el efecto global del síntoma, pero actualmente en MEDEAS-W solo tienen carácter informativo.

a)

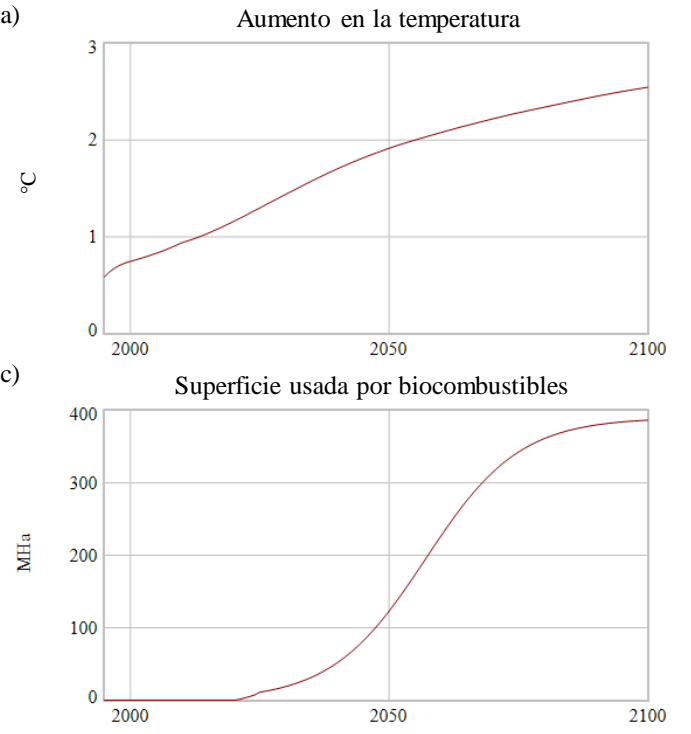

b)

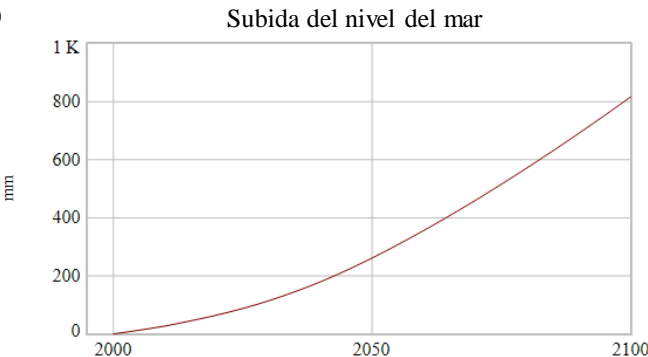

d)

\begin{tabular}{llll}
\multicolumn{3}{c}{ Riesgo de escasez de minerales en 2100} \\
Aluminio & $\bigcirc$ & Manganeso \\
Cadmio & $\bigcirc$ & Molibdeno & $\bigcirc$ \\
Cobre & $\bigcirc$ & Níquel & $\bigcirc$ \\
Cromo & $\bigcirc$ & Plata & $\bigcirc$ \\
Estaño & $\bigcirc$ & Plomo & $\bigcirc$ \\
Galio & $\bigcirc$ & Telurio & $\bigcirc$ \\
Indio & $\bigcirc$ & Vanadio & $\bigcirc$ \\
Litio & $\bigcirc$ & Zinc & \\
Magnesio & $\bigcirc$ &
\end{tabular}

Figura 5.24: Ejemplos de síntomas que se obtienen en MEDEAS-W y muestran un deterioro ambiental del planeta en una simulación del escenario BAU.

Notas: a) aumento de la temperatura $\left({ }^{\circ} \mathrm{C}\right)$, b) subida el nivel del mar (mm), c) superficie usada por biocombustibles (millones de hectáreas) y d) escasez de minerales (rojo: alta; amarilla: media; verde: baja).

Generar los patrones históricos es otra importante prueba de evaluación de los patrones de comportamiento. El modelo MEDEAS-W v2.0 muestra en su periodo histórico un comportamiento muy parecido al mostrados por los datos históricos para las distintas variables del modelo analizadas, como se muestra en la Figura 5.25 para la energía primaria total, la extracción de petróleo convencional, las emisiones de $\mathrm{CO}_{2}$ y la energía primaria obtenida por renovables. 


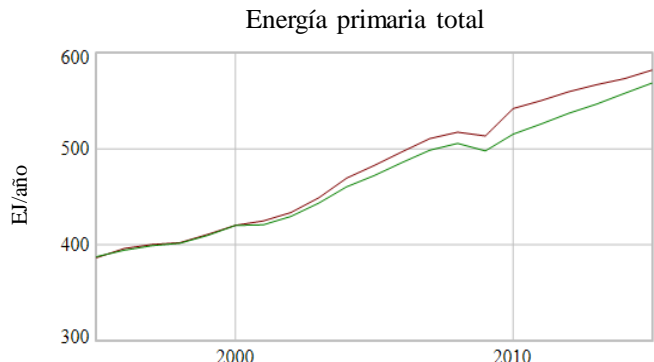

c)

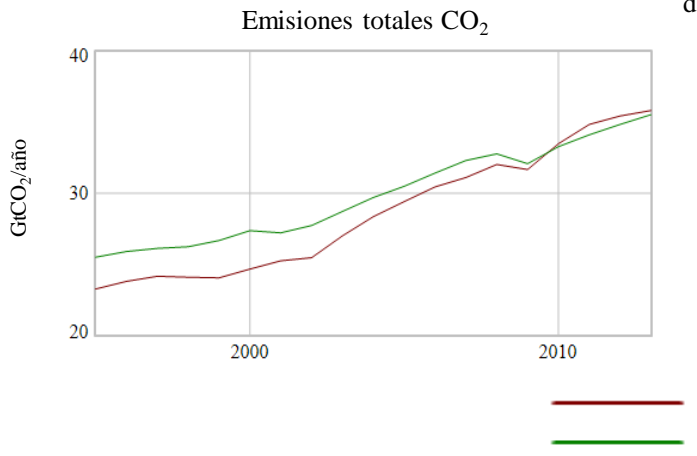

b)

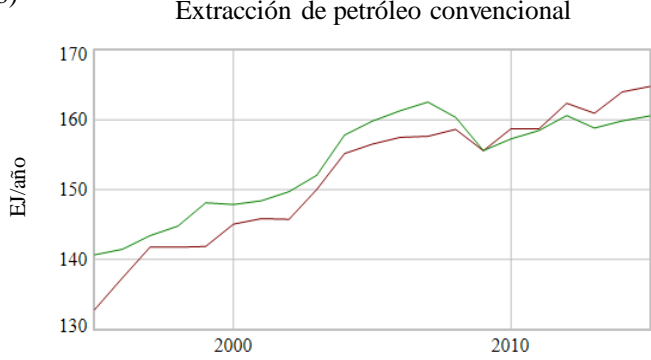

d)

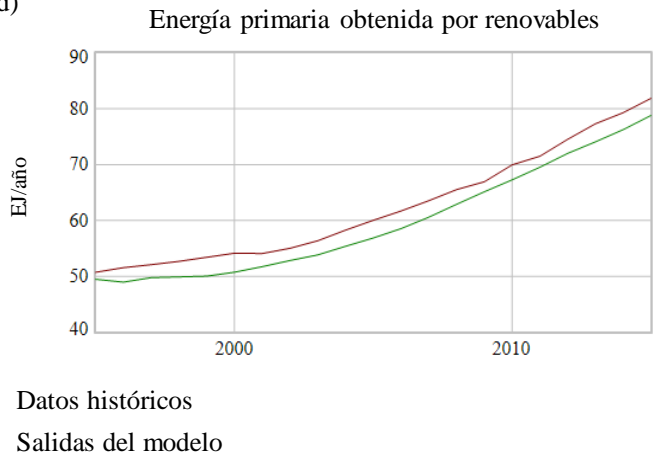

Figura 5.25: Comparación entre el comportamiento obtenido en el modelo y el comportamiento histórico en las variables.

Notas: a) energía primaria total (EJ), b) extracción de petróleo convencional (EJ), c) emisiones de $\mathrm{CO}_{2}$ (gigatoneladas de $\mathrm{CO}_{2}$ ) y d) energía primaria obtenida por renovables (EJ).

Aunque el comportamiento del modelo es muy parecido a los datos históricos, en la Figura 5.25 se puede observar que existen diferencias entre ambos datos. Estas diferencias se deben a las diversas incertidumbres que existen en el modelo como, por ejemplo, en los parámetros, en la estructura o incluso en los datos históricos, que pueden ser diferentes según la fuente utilizada. Como la transparencia es uno de los objetivos más importantes de MEDEAS, las diferencias existentes en el periodo histórico en algunas variables del modelo, como las de la Figura 5.25, se muestran en el propio modelo. Además, es importante reconocer que el modelo MEDEAS-W utiliza para algunas variables datos de su periodo histórico, práctica no recomendable en el modelado en dinámica de sistemas. En esas variables, los datos hasta 2012-2015 son históricos en vez de simulaciones desde el tiempo inicial del modelo, el año 1995. Existen varias razones por la que se ha usado esta práctica, por ejemplo, en el desarrollo de muchas de las energías renovables los datos históricos en el año 1995 son prácticamente nulos, por lo que ha sido necesario utilizar datos históricos hasta 2015 para poder representar mejor su evolución. Otro ejemplo es la utilización de datos históricos en la parte económica del modelo, que ha permitido recrear situaciones como la crisis económica del año 2009. Sin embargo, aunque se han utilizado datos históricos para algunas variables del modelo, esto no ocurre para la gran mayoría de las variables, como las mostradas en la Figura 5.25.

La realización de pruebas de evaluación de los patrones de comportamiento a las predicciones futuras que proporciona el modelo es una tarea muy compleja en un modelo como MEDEAS-W. El uso de observaciones a corto plazo que se puedan validar con los datos de la actualidad no tiene mucho sentido ya que los IAM como MEDEAS-W no están diseñados para capturar la variabilidad a corto plazo, sino para mostrar tendencias a largo plazo. En cuanto a los patrones a largo plazo, su evaluación será también muy complicada ya que, como se ha comentado en el apartado 5.1.3 de validación de los IAM, existen muchas limitaciones. 
Sin embargo, el comportamiento futuro de las variables del modelo se puede analizar gráficamente buscando patrones de comportamiento extraños o imposibles con respecto al comportamiento esperado. También se puede analizar buscando las relaciones entre el comportamiento de distintas variables del modelo. Por ejemplo, no tiene sentido que el PIB siga creciendo si el modelo determina una escasez enorme de energía, o que un aumento de la temperatura global no tenga sus efectos sobre la economía. El modelo MEDEAS-W está formado por miles de variables y gráficamente se ha analizado el comportamiento de muchas de ellas, pudiéndose encontrar distintos ejemplos del análisis del comportamiento de variables en el futuro en los capítulos 3, 4 y 6 de esta tesis.

\section{Comportamiento anómalo del sistema}

Las pruebas de comportamiento anómalo del sistema examinan la importancia de las estructuras del modelo analizando la aparición de comportamientos anómalos cuando la relación de esa estructura con el resto del modelo se borra o se modifica (Sterman, 2000). El análisis del cierre de un bucle es un método utilizado para buscar anomalías de comportamiento: si con el cierre del bucle aparece un comportamiento anómalo en el modelo eso demuestra la importancia del bucle cerrado. El análisis del cierre de un bucle es particularmente eficaz en conjunción con las pruebas de condiciones extremas. Otra prueba de comportamiento anómalo consiste en sustituir una estructura de desequilibrio por una estructura que asuma que ese subsistema está en equilibrio.

En el desarrollo de MEDEAS-W se han introducido en el modelo varios "interruptores" que permiten activar y desactivar bucles para analizar lo que ocurre en distintas partes del modelo. Algunos de los bucles que los "interruptores" permiten activar o desactivar en MEDEAS-W son: los efectos del cambio climático en la economía, la dinamización endógena de las intensidades energéticas y las tablas input-output o el efecto de tasa de retorno energético (EROI) sobre la demanda de energía. Se han realizado análisis del comportamiento al desactivar distintos bucles en MEDEAS. Por ejemplo, Capellán et al., en su artículo metodológico (capítulo 3 de esta tesis), analiza el comportamiento del modelo activando/desactivando los bucles con los efectos de los límites de los recursos energéticos y del cambio climático sobre el crecimiento económico (Capellán-Pérez et al., 2020). Solé et al., analiza las 16 combinaciones posibles al activar y desactivar cuatro bucles: los dos bucles estudiados anteriormente en el artículo de Capellán et al., la dinamización endógena de las intensidades energéticas y el efecto del EROI sobre la demanda de energía (Solé et al., 2020).

En esta tesis se va a mostrar, a modo de ejemplo, el análisis del cierre del bucle del EROI sobre la demanda de energía en el modelo MEDEAS-W v2.0. Como se muestra en el panel de la Figura 5.26, cuando se desactiva el bucle del EROI sobre la demanda de energía, hasta el año 2040 se observa una disminución de la demanda de energía requerida (línea roja en Figura 5.26a) y a su vez el PIB mundial aumenta (línea roja en Figura 5.26b), es decir, el sistema económico consigue crecer con menos energía. Hay que tener en cuenta que, en las dos simulaciones de esta prueba, el resto de hipótesis del modelo que pueden afectar a esta relación, como la mejora tecnológica, son iguales. En las dos simulaciones, las energías renovables aumentan paulatinamente su peso en la producción total de energía (Figura 5.26c) y a su vez se observa como el EROI del sistema baja (Figura 5.26d), ya que se sustituyen recursos fósiles, como el petróleo, con un EROI muy grande, por energías renovables, como la energía solar o los biocombustibles, con un EROI mucho más pequeño. Aparece entonces un comportamiento anómalo en la simulación con el bucle del EROI desactivado: la sustitución de los recursos fósiles por energías renovables no tiene apenas efecto sobre la relación PIB/Energía, que sigue un comportamiento tendencial (línea roja en 
Figura 5.26e). Un cambio tan importante en la energía utilizada (las energías renovables pasan de cubrir el $15 \%$ actual de la energía total a más del $50 \%$ en 2050), teniendo en cuenta que estas energías tienen EROIs muy diferentes, tiene que tener un efecto sobre la relación PIB/Energía, como puede observase cuando el bucle está activado (línea verde en Figura 5.26e). Esta prueba del cierre del bucle nos ha mostrado la importancia del bucle del efecto del EROI sobre la demanda de energía. Los resultados a partir del año 2040 no tienen que ser tomados en cuenta para esta prueba, ya que aparecen otros efectos como la escasez de energía que afectan a la relación PIB/Energía.

a)

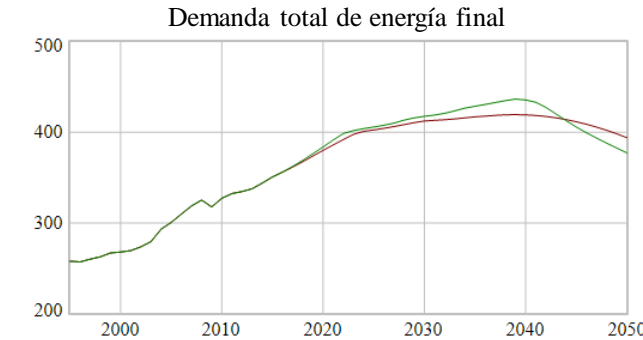

c)

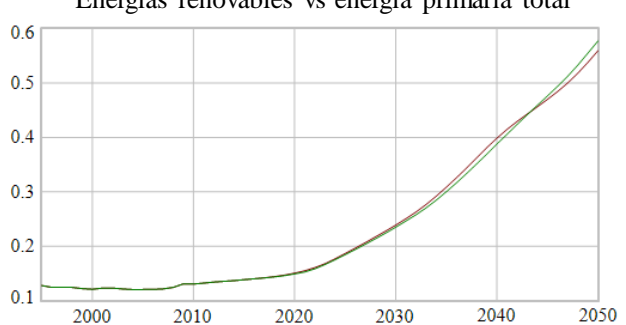

e)

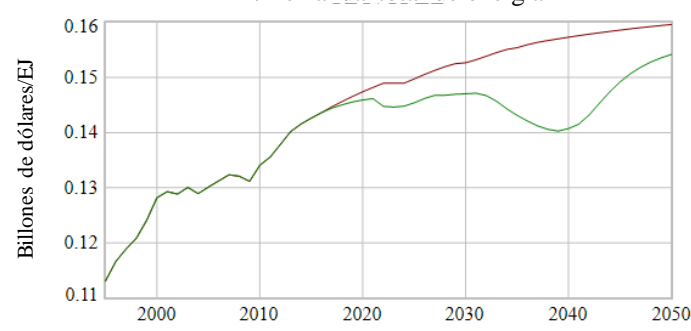

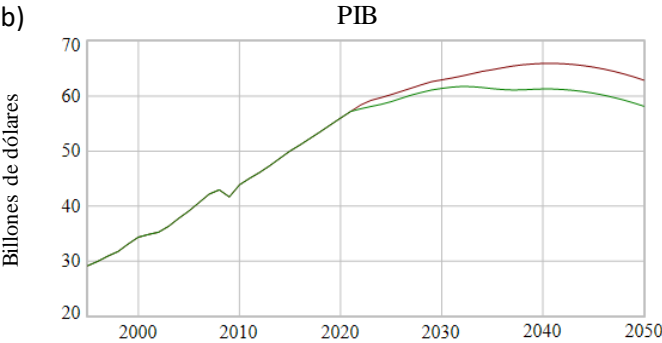

d)

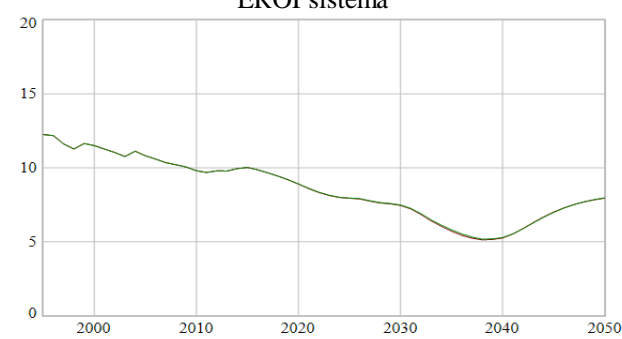

Bucle del EROI desactivado

Bucle del EROI activado

Figura 5.26: Panel con la evolución de distintas variables para dos simulaciones en las que se activa/desactiva el bucle del EROI sobre la demanda de energía en MEDEAS-W v2.0.

Notas: a) demanda total de energía final en EJ, b) PIB en billones de dólares de EEUU del año 1995, c) parte de la energía primaria obtenida por energías renovables, d) EROI del sistema y e) relación entre PIB/demanda total de energía primara en billón de dólares partido EJ.

Un análisis similar se ha realizado en todos los "interruptores" del modelo MEDEAS-W que ha permitido evaluar el efecto de los distintos subsistemas en el modelo.

\section{Comportamiento de distintos miembros de la misma familia}

La prueba de evaluación del comportamiento de distintos miembros de la misma familia consiste en analizar el comportamiento generado por el modelo en otros componentes de la misma clase que el modelo construido. Un modelo no debe explicar solo el comportamiento de un sistema, sino también el de otros sistemas similares con distintas políticas y parámetros (Sterman, 2000). Esta prueba es realmente interesante cuando el modelo obtiene una amplia gama de patrones de 
comportamiento. Por lo general hay que sospechar de un modelo que solo pueda mostrar un único comportamiento.

Para el modelo MEDEAS-W, replicar otro componente de la misma clase es lógicamente imposible, pero como se ha dicho anteriormente, dentro del enfoque MEDEAS se han desarrollado distintos modelos: MEDEAS-EU (Unión Europea), MEDEAS-AUT (Austria) y MEDEAS-BG (Bulgaria) y actualmente se está trabajando en otros modelos como MODESLOW ${ }^{4}$, que será un MEDEAS para España. La estructura de estos modelos es muy parecida, y son relativamente pocas las diferencias, las más importantes son las referidas al comercio internacional, que a nivel mundial no se considera y las referidas al cambio climático, en el que los efectos son globales. Esta prueba es realmente interesante para el caso de MEDEASAUT y MEDEAS-BG en el que los modelos son prácticamente iguales, la diferencia está en los parámetros y políticas aplicadas.

La Figura 5.27 muestra la evolución del PIB per cápita y de las emisiones de $\mathrm{CO}_{2}$ para los cuatro modelos MEDEAS desarrollados hasta ahora. Estos resultados se han obtenido con las siguientes versiones de cada modelo: MEDEAS-AUT v1.2 para Austria, MEDEAS-BG v1.2 para Bulgaria, MEDEAS-EU v1.3 para la Unión Europea y MEDEAS-W v1.4 para el mundo. La figura muestra que el comportamiento de estas dos variables es diferente en los distintos modelos. Por ejemplo, cabe destacar que el PIB per cápita (línea azul) crece durante toda la simulación en Bulgaria mientras que en el resto de regiones antes del año 2050 empieza a decrecer. En cuanto a las emisiones de $\mathrm{CO}_{2}$ (línea naranja), crecen en los cuatro modelos respecto a valores actuales, pero de manera muy diferente: en el año 2050 en Bulgaria las emisiones de $\mathrm{CO}_{2}$ crecen casi un $70 \%$ respecto a las del año 2015, en Austria un 25\%, en el mundo un 5\% y en la Unión Europea un $4 \%$. Lo importante de esta prueba es mostrar como el modelo sirve para estudiar regiones muy diferentes y según los condicionantes de esas regiones, sus parámetros y políticas, el comportamiento de las variables del modelo será muy diferente. Es importante remarcar que los resultados de Austria, Bulgaria y la Unión Europea son todavía preliminares, pues el proceso de desarrollo de estos modelos todavía no ha sido completado.

\footnotetext{
4 Proyecto MODESLOW: Modelización y simulación de escenarios de transición energética hacia una economía baja
} en carbono: el caso español. (ECO2017-85110-R) 

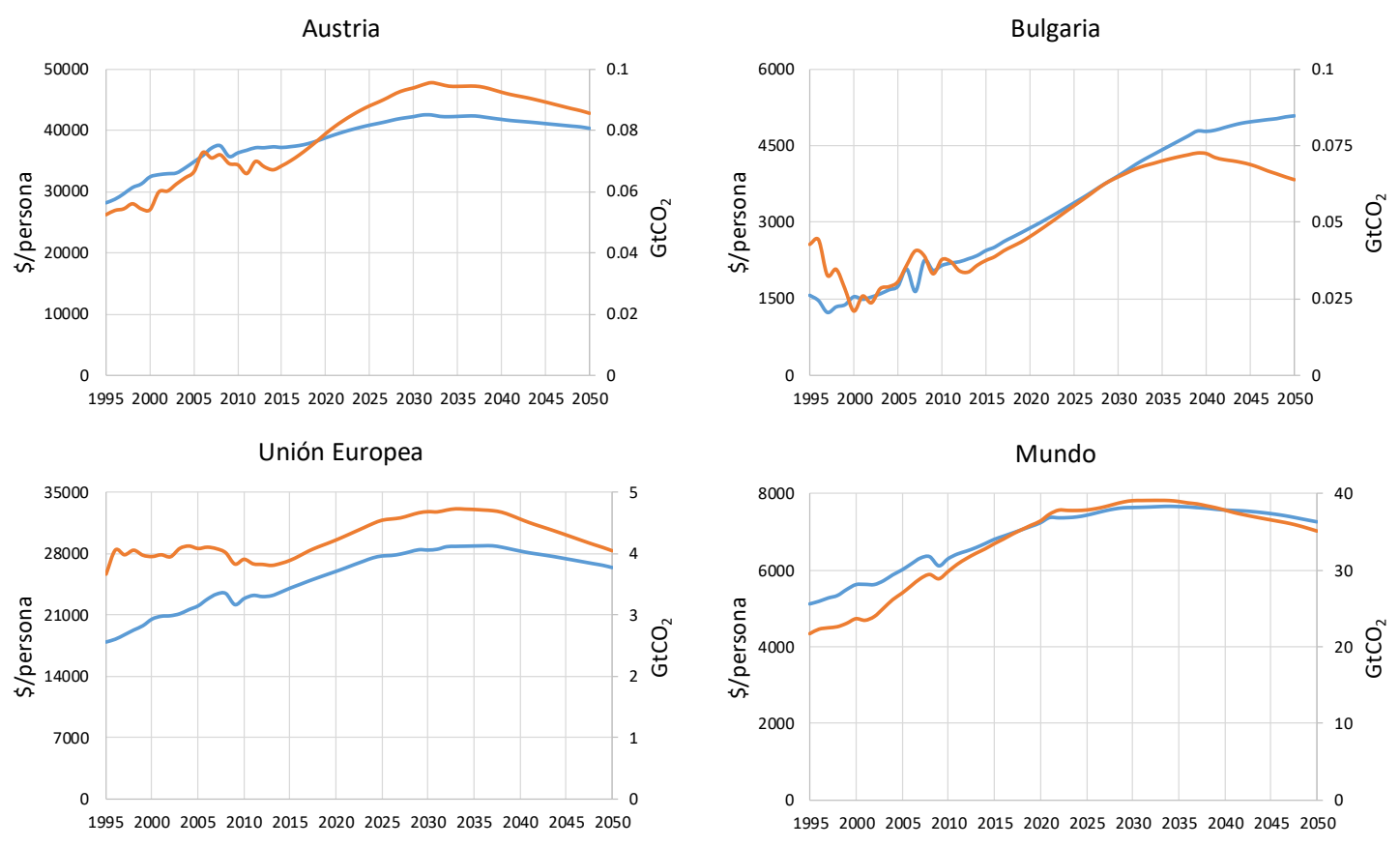

- PIBpc - Emisiones $\mathrm{CO}_{2}$

Figura 5.27: Evolución del PIB per cápita (dólares de EEUU de 1995/persona) y de las emisiones de $\mathrm{CO}_{2}$ (gigatoneladas de $\mathrm{CO}_{2}$ ), para cuatro modelos MEDEAS.

Notas: Se han utilizado los siguientes modelos: MEDEAS-AUT v1.2 para Austria, MEDEAS-BG v1.2 para Bulgaria, MEDEAS-EUv1.3 para la Unión Europea y MEDEAS-Wv1.4 para el mundo.

\section{Comportamiento sorpresa}

Un buen modelo de dinámica de sistemas es probable que muestre comportamientos que están presentes en el sistema real pero que han pasado desapercibidos en el modelo mental (Forrester and Senge, 1980). Las discrepancias entre el comportamiento del modelo y las expectativas que tienen los modeladores o usuarios indican que hay fallos en el modelo formal, en el modelo mental o en ambos. Generalmente son debidos a fallos en el modelo formal, pero algunas veces, sin embargo, son debidos a fallos en el modelo mental. Esta prueba se pasa cuando el modelo genera comportamientos no reconocidos previamente pero que sin embargo ocurren en realidad (Sterman, 2000). Para que la prueba de comportamiento sorpresa sea efectiva, se debe mirar el comportamiento de todas las variables, no solo de los principales resultados, es necesario rastrear todo el modelo buscando comportamientos contraintuitivos o inesperados.

A lo largo del desarrollo de MEDEAS-W se han dado varios comportamientos contraintuitivos, tanto para los desarrolladores del modelo como para los usuarios del mismo. Estos comportamientos han ayudado a comprender mejor el sistema y a utilizar de una manera diferente algunas de las políticas planteadas. Un ejemplo de comportamiento contraintuitivo se ha generado en el modelo MEDEAS-W v1.3.33 al simular una política muy agresiva de electrificación de los vehículos terrestres, que es una de las políticas más planteadas para reducir las emisiones de $\mathrm{CO}_{2}$. Para analizar esta política, dentro de un escenario BAU, se ha aumentado el porcentaje de vehículos eléctricos en el parque total de vehículos (Figura 5.28a). Sin embargo, y como puede observarse en la Figura 5.28b, el aumento del número de vehículos eléctricos no se ve reflejado con una reducción de las emisiones de $\mathrm{CO}_{2}$. De hecho, el comportamiento es contrario, cuanto 
más rápido aumenta el número de vehículos eléctricos, más aumentan las emisiones de $\mathrm{CO}_{2}$. Este comportamiento, en teoría contraintuitivo, es debido a que en un escenario BAU, en el que el crecimiento de las energías renovables se mantiene a niveles actuales, las energías renovables no cubren el 50\% de la electricidad generada hasta aproximadamente el año 2035 (Figura 5.28c). Por tanto, para cubrir el aumento en electricidad generado por el desarrollo de los vehículos eléctricos es necesario aumentar el uso de recursos fósiles como el carbón o el gas. Este aumento implica emitir más emisiones de $\mathrm{CO}_{2}$, es decir, al electrificar el transporte terrestre se aumentan las emisiones de $\mathrm{CO}_{2}$ (ver periodo 2020-2030 en la Figura 5.28b). Estos resultados nos indican que la electrificación del sector transporte debe ir acompañada de un aumento en la electricidad generada por las energías renovables. Un análisis más completo de la electrificación del transporte se encuentra en el trabajo realizado por de Blas et al., (de Blas et al., 2020) y está explicado en el capítulo 6 de esta tesis.

a) Vehículos eléctricos ligeros $4 \mathrm{r}$ respecto al total ligeros $4 \mathrm{r}$

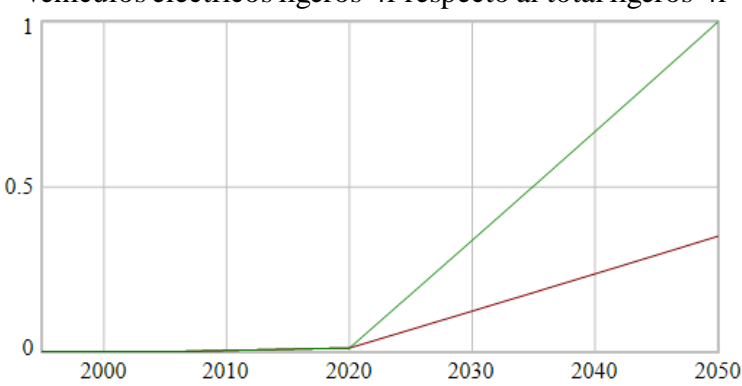

b)

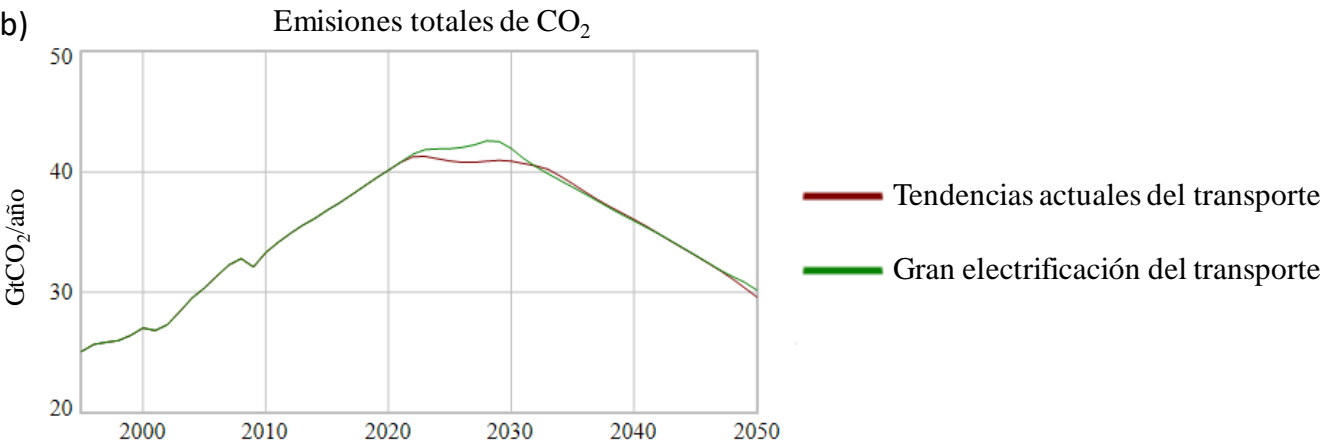

c)

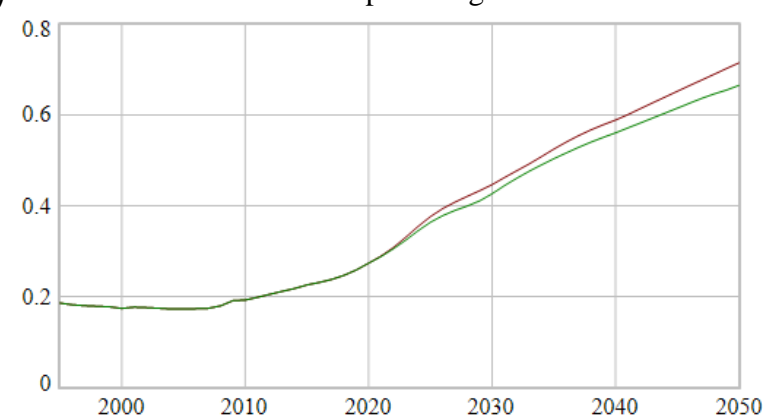

Figura 5.28: Panel con la evolución de distintas variables al mantener las tendencias actuales (línea roja) y en una situación de gran electrificación del transporte (línea verde) en MEDEASWv1.3.33.

Notas: a) evolución de los vehículos ligeros eléctricos de 4 ruedas respecto al total de vehículos ligeros de 4 ruedas, b) evolución de las emisiones totales de $\mathrm{CO}_{2}$ en gigatoneladas de $\mathrm{CO}_{2}$ y c) evolución de la generación total de electricidad que cubren las energías renovables. 4r: vehículos de cuatro ruedas. 
Como se ha explicado con el ejemplo anterior, el modelo MEDEAS-W es capaz de mostrar comportamientos contraintuitivos que ayuden a comprender mejor el sistema real. Otro ejemplo de este tipo de comportamiento mostrado por el modelo MEDEAS-W es que el aumento de la temperatura en el escenario BAU alcanza en el año 2100 un valor por debajo de los $3^{\circ} \mathrm{C}$, siendo este resultado muy diferente al mostrado por el escenario BAU en otros modelos. La razón de este comportamiento diferente radica en los límites y realimentaciones que existen en MEDEASW. Al reducirse la disponibilidad de energía y al realimentar los efectos climáticos sobre la economía, tanto el consumo económico como el energético se reducen, disminuyendo también las emisiones de GEI y, por consiguiente, aumentando en menor medida la temperatura global del planeta en comparación con otros modelos.

\subsubsection{Prueba de mejora del sistema}

El objetivo final del modelado es resolver un problema. La prueba de mejora del sistema pregunta si el proceso de modelado ha contribuido en la resolución del problema ayudando a cambiar el sistema para mejor. Esta prueba consiste en identificar las políticas que conducen a la mejora del sistema, implementarlas en el sistema real y comprobar si el rendimiento del sistema mejora. En la práctica, evaluar el impacto de un modelo es extremadamente difícil. Es complicado también evaluar hasta qué punto el proceso de modelización cambió las creencias de las personas respecto al sistema (Sterman, 2000).

En el caso de MEDEAS-W, los objetivos del modelo son principalmente dos. El primero de ellos es evaluar las políticas a tomar durante los próximos años y facilitar que las conclusiones de dicha evaluación tengan su efecto en las políticas que establezcan las instituciones internacionales. No sabemos si instituciones como la Unión Europea han llegado a tomar decisiones basadas en las conclusiones obtenidas en el modelo MEDEAS-W, pero el hecho de que la Unión Europea haya concedido el proyecto LOCOMOTION (https://www.locomotion-h2020.eu/) con el objetivo de continuar desarrollando el modelo MEDEAS-W implica que se ha hecho un hueco en la comunidad científica y que la Unión Europa es consciente de su utilidad. Se han publicado artículos de MEDEAS en revistas de alto impacto y los artículos han tenido gran repercusión. En cuanto a la parte más técnica de MEDEAS-W, no es posible demostrar por ahora que el uso de las políticas evaluadas en MEDEAS-W han generado en el sistema real el comportamiento que se obtenía en el modelo. La mayoría de políticas que se pueden plantear en MEDEAS-W son a largo plazo (2050 o 2100), por tanto, es necesario que pasen unos años, mínimo hasta el año 2025 o 2030, para evaluar si el comportamiento que muestra el modelo sigue los mismos patrones en la realidad. En el modelo World3 pasaron 30 años desde su publicación para poder demostrar que se estaban cumpliendo en el sistema real las tendencias mostradas por el modelo en su escenario base (Turner, 2008). Además, en el caso de que el comportamiento del sistema coincida con lo planteado en el modelo, esto no implica que haya sido debido concretamente a las políticas planteadas, ese comportamiento puede ser debido a cualquier otra política o suceso. Por tanto, será extremadamente difícil comprobar si realmente el modelo ha ayudado a resolver el problema en este caso.

El otro objetivo principal del modelo MEDEAS-W es facilitar a los ciudadanos la comprensión de la importancia de mantener la sostenibilidad global del planeta, con el doble propósito de concienciarlos sobre los efectos adversos de no mantenerla y ayudarlos a comprender mejor la complejidad de las soluciones a tomar. Evaluar si el modelo cumple este objetivo es igualmente difícil, ya que es muy complicado saber el grado en el que el modelo MEDEAS-W ha servido para concienciar a los ciudadanos de la importancia de mantener la sostenibilidad del planeta. 
Sin embargo, gracias al modelo MEDEAS-W y a las herramientas diseñadas para su explotación y uso, como "Crossroads" (Capellán-Pérez et al., 2019a), sí que podemos saber que el modelo ha llegado a usuarios fuera del ámbito de los desarrolladores. El hecho de que varios profesores de secundaria de la región de Castilla y León estén utilizando MEDEAS-W en sus clases nos indica que, en alguna medida, el modelo les ha ayudado a comprender mejor la complejidad de mantener la sostenibilidad global, y nos asegura que el modelo MEDEAS-W está llegando al menos a una parte de los alumnos de secundaria de la región.

\subsection{Conclusiones del proceso de validación de MEDEAS}

La validación de los modelos de simulación es un proceso complejo que ha generado mucho debate a lo largo de la historia, tanto filosófico en cuanto al sentido del propio concepto de validación, como técnico en cuanto a las pruebas que se pueden realizar para validar un modelo. En esta tesis se entiende por validación al conjunto de pruebas que sirven para mostrar la utilidad de un modelo con el objetivo de resolver un problema.

MEDEAS-W es un modelo de dinámica de sistemas. Para poder generar confianza en sus resultados se han realizado al modelo distintas pruebas de validación. Estas pruebas corresponden a las planteadas por Sterman en el capítulo de su libro dedicado a la validación de modelos de dinámica de sistemas (Sterman, 2000) y son muy similares a las planteadas en otros trabajos de validación en dinámica de sistemas (Barlas, 1996; Forrester and Senge, 1980; Schwaninger and Groesser, 2020).

La realización de las pruebas de validación ha permitido encontrar errores en la estructura del modelo durante su proceso de desarrollo. Pruebas como el análisis de condiciones extremas, el análisis de incertidumbre o el cambio en el método de integración han permitido corregir fallos existentes en el modelo. Las pruebas de patrones de comportamiento han ayudado a comprender mejor el funcionamiento del modelo y, gracias a ello, se han podido realizar modificaciones para mejorar su utilidad. Aunque a partir del proceso de validación se han realizado muchos cambios en el modelo, existen todavía cuestiones detectadas en el proceso de validación que no se han modificado en MEDEAS-W. Por ejemplo, la prueba de adecuación de los límites al submódulo de la demanda de energía ha señalado la importancia de la relación entre las tablas input-output y las intensidades energéticas. La prueba de consistencia en las unidades al modelo MEDEAS-W ha mostrado que es necesario corregir ciertos errores en las unidades, especialmente con las variables adimensionales. La prueba del análisis de sensibilidad ha señalado los parámetros a los que los resultados del modelo son más sensibles, como la relación entre el consumo de energía del sector transporte con su producción económica o la evolución del uso no energético de los recursos fósiles. Los resultados de estas pruebas de validación están guiando el desarrollo actual del modelo dentro del proyecto LOCOMOTION. Mostrar de una manera más detallada la incertidumbre de las variables del modelo es otro de los objetivos planteados en el proyecto LOCOMOTION para mejorar el modelo MEDEAS-W.

Somos conscientes de que el modelo MEDEAS-W tiene muchas limitaciones. Como cualquier modelo difiere de la realidad en muchísimos aspectos, y más siendo este un modelo que trata de representar el sistema económico, energético y medioambiental de manera global. A pesar de las limitaciones existentes, tras el proceso de validación, consideramos que el modelo MEDEAS-W es una buena herramienta a la hora de comprender mejor la importancia de mantener la sostenibilidad global del planeta y de evaluar las políticas que se pueden llevar a cabo para evitar el deterioro medioambiental y sus consecuencias sobre el desarrollo humano. Las principales 
pautas que la literatura propone para validar un modelo se han llevado a cabo en MEDEAS-W. Se ha definido claramente el objetivo del modelo para poder analizar su utilidad, se han realizado de manera iterativa las pruebas de validación dentro del proceso de desarrollo del modelo, se ha realizado un número importante de pruebas de validación en las que no se han encontrado, a juicio de los desarrolladores, motivos que generen una desconfianza en el modelo MEDEAS-W y se ha documentado todo el proceso de validación con las pruebas realizadas al modelo.

Además de intentar cumplir con todas las pautas que la literatura considera en cuanto a validación de modelos de simulación, durante el proceso de desarrollo del modelo MEDEAS-W se han seguido las recomendaciones que el "Grupo de trabajo en evaluación y diagnóstico de los IAM" del IAMC ha ido planteando. El GEEDS de la Universidad de Valladolid forma parte del IAMC desde el año 2018. Uno de los objetivos de este vínculo es incorporar a los modelos MEDEAS los avances que se produzcan en la comunidad científica de los IAM, entre ellos los avances relacionados con validación del modelo.

En el documento de trabajo elaborado por el grupo de evaluación de los IAM del IAMC se establecen cinco aspectos teóricos que deben cumplir todos los IAM (Wilson et al., 2017). Los cinco aspectos son: adecuación, interpretabilidad, verificabilidad, credibilidad y utilidad. MEDEAS-W tiene el objetivo de cumplir los cinco criterios. En cuanto a la adecuación, MEDEAS-W está totalmente orientado para su finalidad, que es ayudar a comprender la importancia de mantener la sostenibilidad global del planeta, para ello considera una gran parte de los problemas medioambientales existentes y permite analizar políticas para su mitigación. La metodología de la dinámica de sistemas permite, con su representación gráfica, que el modelo sea interpretable de una manera relativamente "sencilla" en comparación con otras metodologías, habiéndose desarrollado además un interfaz gráfico para una mejor comunicación de los resultados. El código del modelo está disponible para cualquier usuario en la página web del proyecto (https://www.medeas.eu) y además en los últimos meses se ha realizado un esfuerzo en aumentar la claridad del modelo para una mejor verificabilidad. Gracias a las pruebas de validación realizadas durante todo el proceso de desarrollo, se ha conseguido, a juicio de los desarrolladores, tener un gran nivel de confianza en los resultados que proporciona el modelo. Para aumentar la credibilidad de los usuarios en el modelo, algunas de las pruebas de validación han sido publicadas en la literatura, y el resto han sido documentadas en esta tesis. La utilidad del modelo ha sido en parte demostrada al conceder la Unión Europea el proyecto LOCOMOTION con el objetivo de continuar desarrollando el modelo MEDEAS y también gracias al uso que algunos profesores de secundaria de la región de Castilla y León están dando al modelo con el objetivo de concienciar a sus alumnos de la importancia de mantener la sostenibilidad global.

Wilson et al., proponen en su trabajo ocho métodos para evaluar los IAM, remarcando las ventajas y limitaciones de cada uno de ellos (Wilson et al., 2017). Aunque la validación de MEDEAS-W se ha centrado en las pruebas de validación usadas en la dinámica de sistemas, es importante también utilizar los métodos planteados por el IAMC para los IAM. Algunos de los métodos planteados son muy parecidos a los ya realizados en las pruebas de validación de dinámica de sistemas, como la evaluación de los patrones de comportamiento históricos y de las proyecciones futuras o los análisis de sensibilidad. Sin embargo, hay otros métodos planteados por el IAMC que son muy utilizados para la evaluación de los IAM y que no coinciden con las pruebas ya realizadas al modelo, como son los proyectos de comparación de modelos. Los modelos que se utilizan en los proyectos de comparación suelen ser siempre los mismos, y actualmente se está haciendo un gran esfuerzo en que se utilice MEDEAS-W en esos proyectos. Formar parte del consorcio IAMC puede ayudar a que MEDEAS-W participe en los próximos proyectos de comparación de modelos. 
Por último, hay que recordar que el modelo MEDEAS-W está en continuo desarrollo en los proyectos LOCOMOTION y MODESLOW, y que la validación es una parte dentro del proceso de desarrollo del modelo. Es por ello que, con el objetivo de seguir aumentando la confianza de modeladores y usuarios en MEDEAS-W, se continuarán realizando pruebas de validación al modelo en dichos proyectos. 


\section{PARTE III: APLICACIÓN DE MEDEAS: ANÁLISIS DE POLÍTICAS EN EL SECTOR TRANSPORTE}

\section{Los límites de la descarbonización del transporte en el actual paradigma de crecimiento}

Referencia:

de Blas, I., Mediavilla, M., Capellán-Pérez, I., Duce, C., 2020. The limits of transport decarbonization under the current growth paradigm. Energy Strategy Reviews 32, 100543. https://doi.org/10.1016/j.esr.2020.100543

\section{Resumen:}

Achieving ambitious reductions in greenhouse gases (GHG) is particularly challenging for transportation due to the technical limitations of replacing oil-based fuels. We apply the integrated assessment model MEDEAS-World to study four global transportation decarbonization strategies for 2050. The results show that a massive replacement of oil-fueled individual vehicles to electric ones alone cannot deliver GHG reductions consistent with climate stabilization and could result in the scarcity of some key minerals, such as lithium and magnesium. In addition, energyeconomy feedbacks within an economic growth system create a rebound effect that counters the benefits of substitution. The only strategy that can achieve the objectives globally follows the Degrowth paradigm, combining a quick and radical shift to lighter electric vehicles and nonmotorized modes with a drastic reduction in total transportation demand. 


\section{PARTE IV: CONCLUSIONES}

\section{Conclusiones}

Los impactos que la actividad humana está generando sobre el medio ambiente son cada vez más evidentes. En las últimas décadas, científicos de muy distintos ámbitos han avisado de que si no se modifican los actuales patrones de comportamiento se está poniendo en grave riesgo tanto el futuro de la sociedad humana como del medio ambiente. Con el objetivo de estudiar las complejas relaciones existentes entre las cuestiones socioeconómicas y las medioambientales, se han desarrollado en los últimos 50 años distintos tipos de modelos que simulan escenarios futuros con diferentes alternativas de actuación para ayudar en la toma de decisiones. Estos modelos, denominados modelos de energía-economía-medio ambiente o modelos de evaluación integrada (IAM), son cada vez más utilizados por las principales instituciones y su desarrollo es clave para poder analizar la sostenibilidad y conseguir preservar las condiciones ambientales del planeta que permitan el desarrollo de la sociedad.

El desarrollo de los IAM es una tarea muy compleja, pues integra variables de áreas del conocimiento muy diversas con múltiples realimentaciones y relaciones no lineales. Existen muchos enfoques para intentar resolver estas dificultades, lo que ha generado un conjunto amplio de IAM, cada uno de ellos con sus características y limitaciones. Entre los distintos modelos, WoLiM sigue un enfoque biofísico basado en principios ecológicos y termodinámicos, y utiliza la dinámica de sistemas como metodología para representar las múltiples interrelaciones entre variables.

WoLiM ha servido de base para desarrollar un nuevo modelo más complejo siguiendo su mismo enfoque y utilizando también la dinámica de sistemas. Este modelo, denominado MEDEAS-W por el proyecto europeo homónimo en el que se ha desarrollado, ha tratado de cubrir algunas de las principales limitaciones detectadas en otros IAM. El desarrollo del modelo MEDEAS-W, en colaboración con el resto del grupo de investigación GEEDS, ha sido el trabajo fundamental que ha permitido realizar esta tesis doctoral

Las principales conclusiones de la tesis doctoral están resumidas en la sección 7.1, y están agrupadas en las tres tareas principales de esta tesis, la revisión de los IAM, el desarrollo del modelo MEDEAS-W y la aplicación de MEDEAS-W para estudiar la descarbonización del sector transporte. En la sección 7.2 se plantean algunas de las futuras líneas de trabajo en el desarrollo del modelo tanto en el corto como en el largo plazo. Por último, en la sección 7.3 se indican unas consideraciones generales sobre esta tesis doctoral.

\subsection{Principales conclusiones}

\section{Conclusiones de la revisión de los IAM}

Una profunda revisión de los modelos de evaluación integrada existentes en la literatura ha sido realizada antes de desarrollar un nuevo modelo. Las conclusiones de esta revisión de los IAM, relacionadas con el objetivo 1 de esta tesis, han sido las siguientes:

- Existen decenas de IAM en la literatura con distintos enfoques y estructuras. La gran variedad de modelos es debida a que tanto el objetivo con el que los modelos han sido desarrollados como la metodología utilizada son muy diferentes. Se pueden clasificar en modelos de evaluación o de optimización, recursivos dinámicos o de predicción perfecta, 
modelos de beneficio-coste o modelos de procesos detallados y otras muchas posibles clasificaciones entre modelos.

- Pese a existir muchos IAM, los modelos más utilizados por las principales instituciones como el IPCC o la IEA son casi siempre los mismos. Los modelos AIM, GCAM, IMAGE, MESSAGE, REMIND y WITCH se utilizan en la mayoría de proyectos de comparación de los IAM y también en los principales informes del IPCC. Nuevas herramientas como la enciclopedia online del IAMC y la apertura a nuevos modelos en los futuros informes del IPCC están ayudando a conocer distintas metodologías y modelos.

- La dinámica de sistemas es una metodología utilizada con frecuencia para el desarrollo de los IAM ya que permite representar explícita y dinámicamente las realimentaciones, los retrasos existentes y las no linealidades que predominan en los sistemas complejos. El pionero World3, cuya utilidad ha sido demostrada más de 40 años después, fue desarrollado utilizando la dinámica de sistemas.

- Por su complejidad, los IAM tienen muchas limitaciones. Algunas de las más relevantes son la falta de transparencia en muchos de los modelos, las escasas realimentaciones existentes entre módulos, la predominancia de la parte económica-tecnológica sobre la social o las similitudes en las hipótesis y escenarios utilizados.

\section{Conclusiones del desarrollo del modelo MEDEAS-W}

Con el objetivo de intentar cubrir algunas de las principales limitaciones encontradas en la literatura, se ha desarrollado un nuevo modelo de economía-energía-medio ambiente mediante la colaboración de varios miembros del grupo de investigación GEEDS y que ha sido el marco principal de trabajo de esta tesis. El modelo mundial, MEDEAS-W, da respuesta a las principales cuestiones señaladas en el objetivo 2 de la introducción de esta tesis sobre nuevos modelos de evaluación integrada:

- MEDEAS-W incorpora características que, por lo general, no están desarrolladas en otros IAM. Algunas de las nuevas características son:

- La representación de las limitaciones biofísicas a la disponibilidad de energía y su realimentación sobre la economía.

- El modelado de los requerimientos energéticos y materiales para la transición energética.

- La realimentación de los daños del cambio climático en las variables socioeconómicas.

- La integración de la estructura económica sectorial de manera detallada (análisis input-output).

- Los cambios en el consumo de energía impulsados por una escasez física.

- MEDEAS-W admite la simulación de escenarios muy diferentes mediante la activación de distintos interruptores, opciones que permiten al usuario introducir sus propias hipótesis en el modelo.

- Las potencialidades de MEDEAS-W se han ilustrado mediante la simulación de cuatro casos continuistas (BAU):

- En ausencia de restricciones de disponibilidad de energía y de daños causados por el cambio climático, los resultados obtenidos a lo largo del siglo XXI son, en líneas generales, similares a los obtenidos por los escenarios BAU de otros IAM de la literatura: el PIB per cápita crece unas 4 veces el valor actual, las emisiones de GEI aumentan durante todo el siglo y el cambio en la temperatura es de 3.5$4^{\circ} \mathrm{C}$ por encima de los valores preindustriales. 
- Al activar una de las restricciones anteriores (escasez energética y daños climáticos), o ambas conjuntamente, los resultados se modifican por completo. El modelo muestra una recesión económica persistente durante las próximas décadas con niveles del PIB per cápita similares o inferiores a los actuales. También muestra una gran penetración de las renovables en el mix energético (60-80\%) que impulsa grandes necesidades de minerales, inversiones energéticas y suelo.

- El marco de modelización utilizado para desarrollar el modelo mundial está adaptado para poder utilizarse en otros niveles de agregación regional como la Unión Europea, Austria o Bulgaria. Los resultados preliminares de los modelos en estas regiones muestran que el marco de modelización es adaptable a datos y políticas muy diferentes.

- La realización de las pruebas de validación ha permitido encontrar errores en la estructura del modelo durante su proceso de desarrollo como la existencia de ciertos errores en las unidades, especialmente con las variables adimensionales. A pesar de las limitaciones existentes, el modelo MEDEAS-W es una buena herramienta a la hora de comprender la importancia de mantener la sostenibilidad global y de evaluar las políticas que se pueden llevar a cabo para evitar el deterioro medioambiental del planeta

La estimación de la demanda de energía en los modelos MEDEAS es clave para el análisis de la transición energética y la elaboración de políticas alternativas eficaces. Para su estimación se han utilizado las intensidades energéticas, ya que tienen una definición clara e intuitiva y un cálculo relativamente sencillo en los modelos. Durante el proceso de desarrollo de esta parte de los modelos se han obtenido las siguientes conclusiones, relacionadas con el objetivo 3 de la introducción:

- Estimar la demanda de energía a través de las intensidades energéticas mediante un enfoque "top-down" tiene la ventaja de permitir un análisis más general del sistema, pudiendo capturar complejidades como el efecto rebote en la economía. Por el contrario, este enfoque no es capaz de proporcionar datos con un alto nivel de detalle para un sector determinado. Algunos de los modelos más relevantes en la literatura combinan el enfoque "top-down" con el enfoque "bottom-up" para estimar la demanda energética.

- La evolución de las intensidades energéticas finales depende en gran medida de dos factores: la mejora de la eficiencia energética y la sustitución de un tipo de energía final por otro. A su vez, estos factores están afectados por la presión generada por la escasez energética y por la presión generada por políticas externas, como por ejemplo las debidas al cambio climático. Estas presiones dependen en gran medida de cuestiones sociales como la percepción de la escasez o el factor de olvido.

- La potencialidad del nuevo método se ha puesto de manifiesto en varios casos de estudio en los que se ha mostrado la importancia que tienen variables como la percepción de la escasez energética o el valor máximo de mejora de eficiencia anual sobre las principales salidas del modelo como el PIB per cápita o el consumo de energía final.

- La combinación, en un escenario BAU, de políticas muy agresivas de mejora de eficiencia energética en todos los sectores y, además, una política de electrificación general del sector hogares muestra en MEDEAS-W una reducción de la intensidad energética total en el año 2060 de aproximadamente un 35\% respecto de los valores actuales. Esta reducción es claramente inferior a la mostrada con políticas similares en otros modelos de la literatura. 
- El proceso de validación ha mostrado una importante limitación de esta metodología al no modelar la relación existente entre las intensidades energéticas y los coeficientes de las tablas input-output. A pesar de esta limitación, la validación muestra que la metodología planteada es una buena herramienta para modelar la demanda de energía a través de las intensidades energéticas y, además, posee una gran flexibilidad, pudiéndose adaptar a diferente número de sectores y regiones.

Conclusiones de la aplicación de MEDEAS-W para estudiar la descarbonización del sector $\underline{\text { transporte }}$

El sector transporte es habitualmente identificado como uno de los sectores más difíciles de descarbonizar en la transición hacia una sociedad más sostenible. El modelo MEDEAS-W se ha utilizado para estudiar distintas estrategias para descarbonizar el sector transporte. Las principales conclusiones de este análisis se muestran a continuación (objetivo 4):

- Las tendencias actuales en la descarbonización del sector transporte están muy lejos de poder alcanzar los objetivos de reducción de emisiones de GEI de las principales instituciones nacionales e internacionales, las cuales marcan unos objetivos de hasta un $80 \%$ de reducción en el año 2050 respecto de los valores actuales.

- Escenarios futuros en los que se plantea una rápida sustitución de los vehículos de combustión convencionales por vehículos eléctricos tampoco muestran una reducción de las emisiones a los objetivos marcados, consiguiendo únicamente reducir las emisiones en el año 2050 en torno a un 15\% respecto de los valores actuales.

- El uso masivo de vehículos eléctricos encuentra, además, importantes problemas de disponibilidad en minerales clave como son el litio, el cobre o el manganeso. Las reservas actuales de estos minerales estarían prácticamente agotadas en el año 2050 y una gran parte de sus recursos totales estarían ya extraídos.

- La sustitución de los coches actuales por vehículos muy ligeros como bicicletas o ciclomotores eléctricos ayudaría a reducir una parte de las emisiones de GEI. Los vehículos de gas natural no se pueden considerar como una alternativa viable porque sus emisiones, considerando todo el ciclo de vida del gas natural, son parecidas a la de los vehículos de gasolina o diésel. Los vehículos con biocombustibles tampoco son una alternativa a largo plazo debido a su baja densidad de potencia y a las grandes cantidades de superficie que serían necesarias para cubrir con ellos una parte importante de los vehículos totales.

- La descarbonización del transporte de mercancías con vehículos pesados, así como la del transporte marítimo y aéreo, es mucho más difícil de realizar ya que no existen en la actualidad alternativas eléctricas viables a los vehículos convencionales. Los escenarios simulados en MEDEAS-W muestran que sustituir una parte de las mercancías transportadas en camiones por transporte mediante trenes eléctricos conseguiría reducir en una parte significativa las emisiones del transporte de mercancías.

- Solo el escenario con políticas muy radicales de sustitución de vehículos convencionales por vehículos eléctricos muy ligeros y un ambicioso plan de reciclado de minerales, junto con la reducción de la demanda de transporte, especialmente para el transporte aéreo, es capaz de alcanzar en MEDEAS-W los objetivos de descarbonización del transporte planteados por las principales instituciones. Este escenario se ajusta al paradigma del decrecimiento programado y está totalmente relacionado con cambios en el comportamiento de la sociedad. 


\subsection{Futuras líneas de trabajo}

El objetivo de los IAM es representar todas las relaciones existentes entre la sociedad humana y el medio ambiente, una tarea extremadamente compleja y llena de limitaciones e incertidumbres. El modelo MEDEAS-W, pese a haber cubierto algunas de las limitaciones existentes en el ámbito de los IAM, también contiene diversas limitaciones, algunas de ellas han sido mostradas en el proceso de validación del modelo, capítulo 5 de esta tesis. Corregir estas limitaciones en el corto plazo es una importante línea de trabajo. Además, pese al gran esfuerzo en transparencia realizado en MEDEAS, es posible seguir aumentando la claridad del modelo para aumentar su credibilidad. Mejorar su documentación técnica, establecer una convención de nombres única para sus símbolos y evitar números mágicos en sus ecuaciones son ejemplos de trabajos a corto-medio plazo para seguir mejorando la transparencia del modelo. Desarrollar herramientas de comunicación de los resultados es otro objetivo para conseguir que los resultados y las conclusiones obtenidas en los modelos MEDEAS lleguen a la mayor parte de la sociedad posible.

Por otra parte, en el largo plazo, existen características muy importantes en el sistema que no han sido modeladas en MEDEAS-W. Algunos ejemplos de estas características que guiaran el desarrollo futuro del modelo son:

- El modelado de la intermitencia de las energías renovables a nivel horario.

- La realimentación del consumo de minerales sobre la economía y sobre el desarrollo de las tecnologías renovables.

- El modelado del empleo a través de la demanda de trabajo.

- Las múltiples relaciones de la parte financiera.

- La estimación endógena en el modelo de la población y su división por cohortes de edad.

- La desagregación en el módulo del uso de la tierra en distintos tipos de cultivos y bosques.

El proyecto europeo LOCOMOTION tiene como objetivo continuar con el desarrollo del modelo MEDEAS-W, en dicho proyecto se está trabajando actualmente en añadir algunas de las características anteriores.

Otra futura línea de trabajo a largo plazo es transformar MEDEAS-W en un modelo multirregional para poder analizar las políticas a nivel regional y para poder estudiar los flujos económicos, energéticos y materiales entre, por lo menos, las regiones más importantes del mundo como la UE27, EEUU, China y Rusia. En paralelo, otra línea de trabajo es adaptar el modelo actual a España, este trabajo ya se está realizando dentro del proyecto MODESLOW concedido por el Ministerio de Ciencia e Innovación. Adaptar el modelo a más regiones es otra posible línea de trabajo en el futuro.

Además, en una gran parte de las variables y módulos del modelo, todavía no se han analizado las distintas políticas de actuación, por lo que realizar un estudio detallado de esos módulos que pueda ayudar en la toma de decisiones políticas será otra futura línea de trabajo. En ese sentido, obtener los resultados en el modelo de simular escenarios alternativos como un crecimiento verde o un decrecimiento programado es uno de los principales objetivos a realizar en los próximos meses.

En relación con el ejemplo de aplicación del modelo MEDEAS-W mostrado en esta tesis sobre las distintas estrategias de descarbonización en el sector transporte (capítulo 6), existen también futuras líneas de trabajo. Por una parte, el módulo de transporte de MEDEAS se puede desarrollar con más detalle a través de un enfoque completamente bottom-up en el que, por ejemplo, se defina la demanda de movilidad en cuestión de pasajeros*km o toneladas*km, se clasifiquen los 
desplazamientos por distancia o se introduzcan nuevos tipos de vehículos. Por otra parte, se pueden estudiar nuevas políticas para descarbonizar el transporte como, por ejemplo, sustituir el vehículo privado por el transporte público o la bicicleta, o reducir el número de viajes con medidas como el teletrabajo o el coche compartido.

\subsection{Consideraciones finales}

El modelo MEDEAS-W es una herramienta desarrollada con la finalidad de ayudar a las instituciones internacionales en la evaluación de las políticas a tomar en los próximos años. Además, con el modelo MEDEAS-W se quiere facilitar a los ciudadanos la comprensión de la importancia de mantener la sostenibilidad global del planeta, con el doble propósito de concienciarlos sobre los efectos adversos de no mantenerla y ayudarlos a comprender mejor la complejidad de las soluciones a tomar. El objetivo último de esta tesis doctoral es que el trabajo realizado para desarrollar el modelo y sus aplicaciones sirva realmente a la sociedad en la toma de decisiones para una buena transición energética y para la lucha frente al cambio climático. Por ello, es totalmente necesario que el modelo genere confianza a los posibles usuarios, que sea transparente y que sus resultados sean interpretados correctamente. Aunque esta tesis ha intentado contribuir a este propósito, aún queda mucho trabajo por hacer y mi intención es seguir colaborando en la mejora de los modelos. 


\section{APÉNDICES}

\section{Appendix A: Material suplementario del capítulo 3}

Appendix B: Material suplementario del capítulo 4

Appendix C: Material suplementario del capítulo 6

\section{Referencias}

Abbasi, T., Abbasi, S.A., 2012. Is the Use of Renewable Energy Sources an Answer to the Problems of Global Warming and Pollution? Critical Reviews in Environmental Science and Technology 42, 99-154. https://doi.org/10.1080/10643389.2010.498754

Aboumahboub, T., Auer, C., Bauer, N., Baumstark, L., Bertram, C., Bi, S., Dietrich, J., Dirnaichner, A., Giannousakis, A., Haller, M., Hilaire, J., Klein, D., Koch, J., Körner, A., Kriegler, E., Leimbach, M., Levesque, A., Lorenz, A., Luderer, G., Ludig, S., Lüken, M., Malik, A., Manger, S., Merfort, L., Mouratiadou, I., Pehl, M., Pietzker, R., Piontek, F., Popin, L., Rauner, S., Rodrigues, R., Roming, N., Rottoli, M., Schmidt, E., Schreyer, F., Schultes, A., Sörgel, B., Strefler, J., Ueckerdt, F., 2020. REMIND - REgional Model of INvestments and Development - Version 2.1.0 [WWW Document]. URL https://www.pik-potsdam.de/research/transformation-pathways/models/remind (accessed 9.16.20).

Acemoglu, D., 2008. Introduction to Modern Economic Growth. Princeton University Press, Princetown, New Jersey (USA).

Aghion, P., Howitt, P.W., 2008. The Economics of Growth. MIT Press, Cambridge, Massachusetts \& London, England.

Agora Verkehrwende, Agora Energiewende, Frontier Economics, 2018. The future cost of electricity-based synthetic fuels.

Akhtar, M.K., Wibe, J., Simonovic, S.P., MacGee, J., 2013. Integrated assessment model of society-biosphere-climate-economy-energy system. Environmental Modelling \& Software 49, 1-21. https://doi.org/10.1016/j.envsoft.2013.07.006

Aleklett, K., Höök, M., Jakobsson, K., Lardelli, M., Snowden, S., Söderbergh, B., 2010. The Peak of the Oil Age - Analyzing the world oil production Reference Scenario in World Energy Outlook 2008. Energy Policy 38, 1398-1414. https://doi.org/10.1016/j.enpol.2009.11.021

Alexander, S., Yacoumis, P., 2018. Degrowth, energy descent, and “low-tech" living: Potential pathways for increased resilience in times of crisis. Journal of Cleaner Production, Technology and Degrowth 197, 1840-1848. https://doi.org/10.1016/j.jclepro.2016.09.100

Alexandrov, G.A., Ames, D., Bellocchi, G., Bruen, M., Crout, N., Erechtchoukova, M., Hildebrandt, A., Hoffman, F., Jackisch, C., Khaiter, P., Mannina, G., Matsunaga, T., Purucker, S.T., Rivington, M., Samaniego, L., 2011. Technical assessment and evaluation of environmental models and software: Letter to the Editor. Environmental Modelling \& Software, Thematic issue on the assessment and evaluation of environmental models and software 26, 328-336. https://doi.org/10.1016/j.envsoft.2010.08.004

Álvarez Antelo, D., de Blas Sanz, I., Capellán-Pérez, I., Carpintero Redondo, Ó., De Castro, C., Frechoso, F., Lobejón, L.F., Lomas Huertas, P.L., Mediavilla, M., Miguel, L.J., Nieto, J., Parrado, G., Rodrigo González, P., 2018. D4.3 MEDEAS Model and IOA implementation at country level: the cases of Austria and Bulgaria (Deliverable MEDEAS project, http://www.medeas.eu/deliverables). MEDEAS project, Barcelona, Spain. 
Anandarajah, G., Pye, S., Usher, W., Kesicki, F., Mcglade, C., 2011. TIAM-UCL Global Model Documentation. University College London.

Anderson, K., Broderick, J., 2017. Natural gas and climate change. Manchester: University of Manchester 27, 733-735.

Anderson, K., Peters, G., 2016. The trouble with negative emissions. Science 354, 182-183. https://doi.org/10.1126/science.aah4567

Anthoff, D., Tol, R.S.J., 2014. FUND Model TECHNICAL DESCRIPTION, VERSION 3.9.

Arigoni Ortiz, R., Markandya, A., 2009. Integrated Impact Assessment Models of Climate Change with an Emphasis on Damage Functions: a Literature Review (BC3 WORKING PAPER SERIES). Basque Centre for Climate Change (BC3).

Arto, I., Andreoni, V., Rueda-Cantuche, J.M., 2016a. Global use of water resources: A multiregional analysis of water use, water footprint and water trade balance. Water Resources and Economics 15, 1-14. https://doi.org/10.1016/j.wre.2016.04.002

Arto, I., Capellán-Pérez, I., Lago, R., Bueno, G., Bermejo, R., 2016b. The energy requirements of a developed world. Energy for Sustainable Development 33, 1-13. https://doi.org/10.1016/j.esd.2016.04.001

ASPO, 2009. ASPO Newsletter n. 100 (No. 100). The Association for the Study of Peak Oil and gas.

Ayres, R.U., 2007. On the practical limits to substitution. Ecological Economics 61, 115-128. https://doi.org/10.1016/j.ecolecon.2006.02.011

Ayres, R.U., van den Bergh, J.C.J.M., Lindenberger, D., Warr, B., 2013. The underestimated contribution of energy to economic growth. Structural Change and Economic Dynamics 27, 79-88. https://doi.org/10.1016/j.strueco.2013.07.004

Balci, O., 1994. Validation, verification, and testing techniques throughout the life cycle of a simulation study. Ann Oper Res 53, 121-173. https://doi.org/10.1007/BF02136828

Balcombe, P., Anderson, K., Speirs, J., Brandon, N., Hawkes, A., 2017. The Natural Gas Supply Chain: The Importance of Methane and Carbon Dioxide Emissions. ACS Sustainable Chem. Eng. 5, 3-20. https://doi.org/10.1021/acssuschemeng.6b00144

Bardi, U., 2014. Extracted: How the Quest for Mineral Wealth Is Plundering the Planet. Chelsea Green Publishing, White River Junction, Vermont.

Bardi, U., 2011. The limits to growth revisited. Springer, New York.

Barlas, Y., 1996. Formal aspects of model validity and validation in system dynamics. System Dynamics Review 12, 183-210. https://doi.org/10.1002/(SICI)1099-

1727(199623)12:3<183::AID-SDR103>3.0.CO;2-4

Barlas, Y., Carpenter, S., 1990. Philosophical roots of model validation: Two paradigms. System Dynamics Review 6, 148-166. https://doi.org/10.1002/sdr.4260060203

Barnes, J., Dove, M., Lahsen, M., Mathews, A., McElwee, P., McIntosh, R., Moore, F., O'Reilly, J., Orlove, B., Puri, R., Weiss, H., Yager, K., 2013. Contribution of anthropology to the study of climate change. Nature Climate Change 3, 541-544. https://doi.org/10.1038/nclimate1775

Barnett, H., Morse, C., 1963. Scarcity and Growth. The Economics of Natural Resource Availability. John Hopkins Press, Balitmore.

Barnhart, C.J., Benson, S.M., 2013. On the importance of reducing the energetic and material demands of electrical energy storage. Energy Environ. Sci 6, 1083-1092. https://doi.org/10.1039/C3EE24040A

Barnosky, A.D., Hadly, E.A., Bascompte, J., Berlow, E.L., Brown, J.H., Fortelius, M., Getz, W.M., Harte, J., Hastings, A., Marquet, P.A., Martínez, N.D., Mooers, A., Roopnarine, P., Vermeij, G., Williams, J.W., Gillespie, R., Kitzes, J., Marshall, C., Matzke, N., Mindell, D.P., Revilla, E., Smith, A.B., 2012. Approaching a state shift in Earth/'s biosphere. Nature 486, 52-58. https://doi.org/10.1038/nature11018

Bauer, N., Rose, S.K., Fujimori, S., van Vuuren, D.P., Weyant, J., Wise, M., Cui, Y., Daioglou, V., Gidden, M.J., Kato, E., Kitous, A., Leblanc, F., Sands, R., Sano, F., Strefler, J., Tsutsui, J., 
Bibas, R., Fricko, O., Hasegawa, T., Klein, D., Kurosawa, A., Mima, S., Muratori, M., 2018. Global energy sector emission reductions and bioenergy use: overview of the bioenergy demand phase of the EMF-33 model comparison. Climatic Change. https://doi.org/10.1007/s10584-018-2226-y

Bednar, J., Obersteiner, M., Wagner, F., 2019. On the financial viability of negative emissions. Nature Communications 10, 1-4. https://doi.org/10.1038/s41467-019-09782-x

Bernard, A., Vielle, M., 2008. GEMINI-E3, a general equilibrium model of international-national interactions between economy, energy and the environment. CMS 5, 173-206. https://doi.org/10.1007/s10287-007-0047-y

Bhattacharyya, S.C.T., Govinda R., 2009. Energy Demand Models For Policy Formulation: A Comparative Study Of Energy Demand Models, Policy Research Working Papers. The World Bank.

Bibas, R., Cassen, C., Crassous, R., Guivarch, C., Hamdi-Cherif, M., Hourcade, J.C., Leblanc, F., Mejean, A., Ó Broin, E., Rozenberg, J., Sassi, O., Vogt-Schilb, A., Waisman, H., 2016. IMACLIM-R World 1.1 model documentation. CNRS-CIRED, Nogent-sur-Marne (France).

Blanford, G.J., Kriegler, E., Tavoni, M., 2014. Harmonization vs. fragmentation: overview of climate policy scenarios in EMF27. Climatic Change 123, 383-396. https://doi.org/10.1007/s10584-013-0951-9

Bloomberg, 2018. New Energy Outlook 2018. New York (USA).

Bosetti, V., Carraro, C., Galeotti, M., Massetti, E., Tavoni, M., 2006. WITCH. A World Induced Technical Change Hybrid Model (Working Paper No. 2006_46). Department of Economics, University of Venice "Ca' Foscari."

Bovari, E., Giraud, G., Mc Isaac, F., 2018. Coping With Collapse: A Stock-Flow Consistent Monetary Macrodynamics of Global Warming. Ecological Economics 147, 383-398. https://doi.org/10.1016/j.ecolecon.2018.01.034

BP, 2020. Statistical Review of World Energy [WWW Document]. bp global. URL https://www.bp.com/en/global/corporate/energy-economics/statistical-review-ofworld-energy.html (accessed 1.8.21).

BP, 2016. BP Statistical Review of World Energy June 2016, Statistical Review of World Energy. British Petroleum.

Brandt, A.R., 2017. How Does Energy Resource Depletion Affect Prosperity? Mathematics of a Minimum Energy Return on Investment (EROI). Biophys Econ Resour Qual 2, 2. https://doi.org/10.1007/s41247-017-0019-y

Brandt, A.R., Farrell, A.E., 2007. Scraping the bottom of the barrel: greenhouse gas emission consequences of a transition to low-quality and synthetic petroleum resources. Climatic Change 84, 241-263. https://doi.org/10.1007/s10584-007-9275-y

Brecha, R.J., 2012. Logistic curves, extraction costs and effective peak oil. Energy Policy 51, 586-597. https://doi.org/10.1016/j.enpol.2012.09.016

Brunke, J.-C., Blesl, M., 2014. Energy conservation measures for the German cement industry and their ability to compensate for rising energy-related production costs. Journal of Cleaner Production 82, 94-111. https://doi.org/10.1016/j.jclepro.2014.06.074

Bumby, S., Druzhinina, E., Feraldi, R., Werthmann, D., Geyer, R., Sahl, J., 2010. Life Cycle Assessment of Overhead and Underground Primary Power Distribution. Environ. Sci. Technol. 44, 5587-5593. https://doi.org/10.1021/es9037879

Bunsen, T., Cazzola, P., Gorner, M., Paoli, L., Scheffer, S., Schuitmaker, R., Tattini, J., Teter, J., 2018. Global EV Outlook 2018: Towards cross-modal electrification. International Energy Agency.

Burke, M., Hsiang, S.M., Miguel, E., 2015. Global non-linear effect of temperature on economic production. Nature 527, 235-239. https://doi.org/10.1038/nature15725

Burniaux, J.-M., Truong, T.P., 2002. GTAP-E: An Energy-Environmental Version of the GTAP Model. Center for Global Trade Analysis, Indiana. 
Calvin, K., Clarke, L., Edmonds, J., Eom, J., Hejazi, M., Kim, S., Kyle, P., Link, R., Luckow, P., Patel, P., 2020. GCAM v5.3 Documentation: Global Change Assessment Model (GCAM) [WWW Document]. URL http://jgcri.github.io/gcam-doc/index.html (accessed 6.20.19).

Calvin, K., Wise, M., Kyle, P., Clarke, L., Edmonds, J., 2017. A HINDCAST EXPERIMENT USING THE GCAM 3.0 AGRICULTURE AND LAND-USE MODULE. Climate Change Economics. https://doi.org/10.1142/S2010007817500051

Calvo, G., Mudd, G., Valero, A., Valero, A., 2016. Decreasing Ore Grades in Global Metallic Mining: A Theoretical Issue or a Global Reality? Resources 5, 36. https://doi.org/10.3390/resources5040036

Campbell, C.J., Laherrère, J., 1998. The end of cheap oil. Scientific American 278, 60-65.

Capellán-Pérez, I., 2016. Development and application of environmental integrated assessment modelling towards sustainability (Thesis). University of the Basque Country, Bilbao, Spain.

Capellán-Pérez, I., Álvarez-Antelo, D., Miguel, L.J., 2019a. Global Sustainability Crossroads: A Participatory Simulation Game to Educate in the Energy and Sustainability Challenges of the 21st Century. Sustainability 11, 3672. https://doi.org/10.3390/su11133672

Capellán-Pérez, I., Arto, I., Polanco-Martínez, J.M., González-Eguino, M., Neumann, M.B., 2016. Likelihood of climate change pathways under uncertainty on fossil fuel resource availability. Energy Environ. Sci 9, 2482-2496. https://doi.org/10.1039/C6EE01008C

Capellán-Pérez, I., Blas, I. de, Nieto, J., Castro, C. de, Miguel, L.J., Carpintero, Ó., Mediavilla, M., Lobejón, L.F., Ferreras-Alonso, N., Rodrigo, P., Frechoso, F., Álvarez-Antelo, D., 2020. MEDEAS: a new modeling framework integrating global biophysical and socioeconomic constraints. Energy Environ. Sci. https://doi.org/10.1039/C9EE02627D

Capellán-Pérez, I., de Blas, I., Nieto, J., De Castro, C., Miguel, L.J., Mediavilla, M., Carpintero, Ó., Rodrigo, P., Frechoso, F., Cáceres, S., 2017a. D4.1 MEDEAS Model and IOA implementation at global geographical level (Deliverable MEDEAS project, http://www.medeas.eu/deliverables No. D4.1). MEDEAS project, Barcelona, Spain.

Capellán-Pérez, I., De Castro, C., 2019. Modelling of stochastic climate damages consistent with global Climate Emergency. Under review.

Capellán-Pérez, I., de Castro, C., 2017. Integration of global environmental change threat to human societies in energy-economy-environment models. Presented at the 12th Conference of the European Society for Ecological Economics, Budapest (Hungary).

Capellán-Pérez, I., de Castro, C., Arto, I., 2017b. Assessing vulnerabilities and limits in the transition to renewable energies: Land requirements under $100 \%$ solar energy scenarios. Renewable and Sustainable Energy Reviews 77, 760-782. https://doi.org/10.1016/j.rser.2017.03.137

Capellán-Pérez, I., de Castro, C., Mediavilla, M., Miguel, L.J., de Blas, I., 2017c. WoLiM_1.5 Model [WWW Document]. URL https://www.researchgate.net/publication/313823551_WoLiM_15_Model_Document ation

Capellán-Pérez, I., de Castro, C., Miguel González, L.J., 2019b. Dynamic Energy Return on Energy Investment (EROI) and material requirements in scenarios of global transition to renewable energies. Energy Strategy Reviews 26, 100399. https://doi.org/10.1016/j.esr.2019.100399

Capellán-Pérez, I., Mediavilla, M., Castro, C. de, Carpintero, Ó., Miguel, L.J., 2015. More growth? An unfeasible option to overcome critical energy constraints and climate change. Sustain Sci 10, 397-411. https://doi.org/10.1007/s11625-015-0299-3

Capellán-Pérez, I., Mediavilla, M., de Castro, C., Carpintero, Ó., Miguel, L.J., 2014. Fossil fuel depletion and socio-economic scenarios: An integrated approach. Energy 77, 641-666. https://doi.org/10.1016/j.energy.2014.09.063 
Capros, P., Van Regemorter, D., Paroussos, L., Karkatsoulis, P., 2013. GEM-E3 Model Documentation. Joint Research Centre.

Carbajales-Dale, M., Barnhart, C.J., Brandt, A.R., Benson, S.M., 2014. A better currency for investing in a sustainable future. Nature Clim. Change 4, 524-527. https://doi.org/10.1038/nclimate2285

Carrara, S., Longden, T., 2017. Freight futures: The potential impact of road freight on climate policy. Transportation Research Part D: Transport and Environment 55, 359-372. https://doi.org/10.1016/j.trd.2016.10.007

Carrington, G., Stephenson, J., 2018. The politics of energy scenarios: Are International Energy Agency and other conservative projections hampering the renewable energy transition? Energy Research \& Social Science 46, 103-113. https://doi.org/10.1016/j.erss.2018.07.011

Château, J., Dellink, R., Lanzi, E., 2014. An Overview of the OECD ENV-Linkages Model: Version 35. https://doi.org/10.1787/5jz2qck2b2vd-en

Chen, M., Ma, X., Chen, B., Arsenault, R., Karlson, P., Simon, N., Wang, Y., 2019. Recycling Endof-Life Electric Vehicle Lithium-Ion Batteries. Joule 3, 2622-2646. https://doi.org/10.1016/j.joule.2019.09.014

Christensen, P.P., 1989. Historical roots for ecological economics - Biophysical versus allocative approaches. Ecological Economics 1, 17-36. https://doi.org/10.1016/09218009(89)90022-0

Clack, C.T.M., Qvist, S.A., Apt, J., Bazilian, M., Brandt, A.R., Caldeira, K., Davis, S.J., Diakov, V., Handschy, M.A., Hines, P.D.H., Jaramillo, P., Kammen, D.M., Long, J.C.S., Morgan, M.G., Reed, A., Sivaram, V., Sweeney, J., Tynan, G.R., Victor, D.G., Weyant, J.P., Whitacre, J.F., 2017. Evaluation of a proposal for reliable low-cost grid power with $100 \%$ wind, water, and solar. PNAS 114, 6722-6727. https://doi.org/10.1073/pnas.1610381114

Cleveland, C., Stern, D., 2001. Natural resource scarcity indicators: an ecological economic synthesis, in: The Economics of Nature and the Nature of Economics (Eds Cleveland, Cutler J., Robert Costanza and David I. Stern). Edward Elgar Publishing, Cheltenham, UK, pp. 238-261.

Cleveland, C.J., 1991. Natural resource scarcity and economic growth revisited: Economic and biophysical perspectives. Ecological Economics: The Science and Management of Sustainability 289-317.

Cochran, T.B., Feiveson, H.A., Patterson, W., Pshakin, G., Ramana, M., Schneider, M., Suzuki, T., von Hippel, F., 2010. Fast breeder reactor programs: history and status. International Panel on Fissile Materials.

Cole, H.S., Freeman, C., Jahoda, M., 1976. Models of Doom. A Critique of the Limits to Growth. Population 31, 1327. https://doi.org/10.2307/1531545

Cosme, I., Santos, R., O'Neill, D.W., 2017. Assessing the degrowth discourse: A review and analysis of academic degrowth policy proposals. Journal of Cleaner Production 149, 321-334. https://doi.org/10.1016/j.jclepro.2017.02.016

Costanza, R., 1991. Ecological Economics: The Science and Management of Sustainability, Revised ed. edition. ed. Columbia University Press, New York.

Costanza, R., 1989. What is Ecological Economics? Ecological Economics 1, 1-17.

Costanza, R., Kubiszewski, I., Giovannini, E., Lovins, H., McGlade, J., Pickett, K.E., Ragnarsdóttir, K.V., Roberts, D., De Vogli, R., Wilkinson, R., 2014. Development: Time to leave GDP behind. Nature 505, 283-285. https://doi.org/10.1038/505283a

Cottrell, F., 1955. Energy and society: the relation between energy, social changes, and economic development. McGraw-Hill.

Creutzig, F., Jochem, P., Edelenbosch, O.Y., Mattauch, L., Vuuren, D.P. van, McCollum, D., Minx, J., 2015. Transport: A roadblock to climate change mitigation? Science 350, 911-912. https://doi.org/10.1126/science.aac8033 
Crownshaw, T., Morgan, C., Adams, A., Sers, M., Britto dos Santos, N., Damiano, A., Gilbert, L., Yahya Haage, G., Horen Greenford, D., 2018. Over the horizon: Exploring the conditions of a post-growth world. The Anthropocene Review 2053019618820350. https://doi.org/10.1177/2053019618820350

Cruz, L., Dias, J., 2016. Energy and CO2 intensity changes in the EU-27: Decompositon into explanatory effects.

Cumming, G.S., Alcamo, J., Sala, O., Swart, R., Bennett, E.M., Zurek, M., 2005. Are Existing Global Scenarios Consistent with Ecological Feedbacks? Ecosystems 8, 143-152. https://doi.org/10.1007/s10021-004-0075-1

Cunha, B.S.L., Garaffa, R., Gurgel, A.C., 2019. TEA Model Documentation (http://bibliotecadigital.fgv.br/dspace/bitstream/handle/10438/28756/TD\%20520\%20 -\%20FGVAGRO.pdf?sequence=1\&isAllowed=y).

Dafermos, Y., Papatheodorou, C., 2015. Linking functional with personal income distribution: a stock-flow consistent approach. International Review of Applied Economics 29, 787815. https://doi.org/10.1080/02692171.2015.1054365

Daily, G., 1997. Nature's services: societal dependence on natural ecosystems, Island Press. ed. Island Press, Washington DC (USA).

Dale, M., Krumdieck, S., Bodger, P., 2012a. Global energy modelling - A biophysical approach (GEMBA) Part 2: Methodology. Ecological Economics 73, 158-167. https://doi.org/10.1016/j.ecolecon.2011.10.028

Dale, M., Krumdieck, S., Bodger, P., 2012b. Global energy modelling - A biophysical approach (GEMBA) part 1: An overview of biophysical economics. Ecological Economics 73, 152157. https://doi.org/10.1016/j.ecolecon.2011.10.014

Dale, M., Krumdieck, S., Bodger, P., 2011. A Dynamic Function for Energy Return on Investment. Sustainability 3, 1972-1985. https://doi.org/10.3390/su3101972

D'Alessandro, S., Luzzati, T., Morroni, M., 2010. Energy transition towards economic and environmental sustainability: feasible paths and policy implications. Journal of Cleaner Production 18, 291-298. https://doi.org/10.1016/j.jclepro.2009.10.015

Daly, H., 1968. On economics as a life science. Journal of Political Economy 76, 392-406.

Danielsen, F., Beukema, H., Burgess, N.D., Parish, F., Brühl, C.A., Donald, P.F., Murdiyarso, D., Phalan, B., Reijnders, L., Struebig, M., Fitzherbert, E.B., 2009. Biofuel Plantations on Forested Lands: Double Jeopardy for Biodiversity and Climate. Conservation Biology 23, 348-358. https://doi.org/10.1111/j.1523-1739.2008.01096.x

Daudey, E., García-Peñalosa, C., 2007. The personal and the factor distributions of income in a cross-section of countries. The Journal of Development Studies 43, 812-829. https://doi.org/10.1080/00220380701384406

de Blas, I., Mediavilla, M., Capellán-Pérez, I., Duce, C., 2020. The limits of transport decarbonization under the current growth paradigm. Energy Strategy Reviews 32, 100543. https://doi.org/10.1016/j.esr.2020.100543

de Blas, I., Miguel, L.J., Capellán-Pérez, I., 2019. Modelling of sectoral energy demand through energy intensities in MEDEAS integrated assessment model. Energy Strategy Reviews 26, 100419. https://doi.org/10.1016/j.esr.2019.100419

de Blas Sanz, I., Capellán-Pérez, I., Carpintero Redondo, Ó., De Castro, C., Frechoso, F., Lobejón, L.F., Lomas Huertas, P.L., Mediavilla, M., Miguel, L.J., Nieto, J., Rodrigo, P., 2018. D4.2 MEDEAS Model and IOA implementation at European geographical level (Deliverable MEDEAS project, http://www.medeas.eu/deliverables). MEDEAS project, Barcelona, Spain.

de Castro, C., Capellán-Pérez, I., 2018. Concentrated Solar Power: Actual Performance and Foreseeable Future in High Penetration Scenarios of Renewable Energies. Biophys Econ Resour Qual 3, 14. https://doi.org/10.1007/s41247-018-0043-6 
de Castro, C., Carpintero, Ó., Frechoso, F., Mediavilla, M., de Miguel, L.J., 2014. A top-down approach to assess physical and ecological limits of biofuels. Energy 64, 506-512. https://doi.org/10.1016/j.energy.2013.10.049

de Castro, C., Mediavilla, M., Miguel, L.J., Frechoso, F., 2013. Global solar electric potential: A review of their technical and sustainable limits. Renewable and Sustainable Energy Reviews 28, 824-835. https://doi.org/10.1016/j.rser.2013.08.040

de Castro, C., Mediavilla, M., Miguel, L.J., Frechoso, F., 2011. Global wind power potential: Physical and technological limits. Energy Policy, Sustainability of biofuels 39, 66776682. https://doi.org/10.1016/j.enpol.2011.06.027

de Castro, C., Miguel, L.J., Mediavilla, M., 2009. The role of non conventional oil in the attenuation of peak oil. Energy Policy 37, 1825-1833. https://doi.org/10.1016/j.enpol.2009.01.022

De Haan, M., 2001. A Structural Decomposition Analysis of Pollution in the Netherlands. Economic Systems Research 13, 181-196. https://doi.org/http://dx.doi.org/10.1080/09537320120052452

de Koning, A., Kleijn, R., Huppes, G., Sprecher, B., van Engelen, G., Tukker, A., 2018. Metal supply constraints for a low-carbon economy? Resources, Conservation and Recycling 129, 202-208. https://doi.org/10.1016/j.resconrec.2017.10.040

DeCicco, J.M., Liu, D.Y., Heo, J., Krishnan, R., Kurthen, A., Wang, L., 2016. Carbon balance effects of U.S. biofuel production and use. Climatic Change 138, 667-680. https://doi.org/10.1007/s10584-016-1764-4

Delogu, M., Zanchi, L., Dattilo, C.A., Pierini, M., 2017. Innovative composites and hybrid materials for electric vehicles lightweight design in a sustainability perspective. https://doi.org/10.1016/j.mtcomm.2017.09.012

Després, J., Keramidas, K., Schmitz, A., Kitous, A., Schade, B., 2018. POLES-JRC model documentation (https://publications.jrc.ec.europa.eu/repository/bitstream/JRC113757/kjna29454enn .pdf). Joint Research Centre.

Diaz, D., Moore, F., 2017. Quantifying the economic risks of climate change. Nature Climate Change 7, 774-782. https://doi.org/10.1038/nclimate3411

Dietz, S., Stern, N., 2015. Endogenous Growth, Convexity of Damage and Climate Risk: How Nordhaus' Framework Supports Deep Cuts in Carbon Emissions. The Economic Journal 125, 574-620.

Dietz, T., Gardner, G.T., Gilligan, J., Stern, P.C., Vandenbergh, M.P., 2009. Household actions can provide a behavioral wedge to rapidly reduce US carbon emissions. PNAS 106, 18452-18456. https://doi.org/10.1073/pnas.0908738106

Dietzenbacher, E., Los, B., Stehrer, R., Timmer, M., de Vries, G., 2013. The Construction of World Input-Output Tables in the WIOD Project. Economic Systems Research 25, 7198. https://doi.org/10.1080/09535314.2012.761180

Duesenfeld, 2020. Savings of 4.8 t CO2 per ton of recycled batteries - Duesenfeld [WWW Document]. URL https://www.duesenfeld.com/recycling_en.html (accessed 6.10.20).

Duesenfeld, 2019. Duesenfeld | Recycling high-voltage lithium-ion energy storage systems efficiently [WWW Document]. URL https://www.duesenfeld.com/efficiency.html (accessed 11.29.19).

Dunn, J.B., Gaines, L., Sullivan, J., Wang, M.Q., 2012. Impact of Recycling on Cradle-to-Gate Energy Consumption and Greenhouse Gas Emissions of Automotive Lithium-Ion Batteries. Environ. Sci. Technol. 46, 12704-12710. https://doi.org/10.1021/es302420z

Dupont, E., Koppelaar, R., Jeanmart, H., 2020. Global available solar energy under physical and energy return on investment constraints. Applied Energy 257, 113968. https://doi.org/10.1016/j.apenergy.2019.113968 
Dupont, E., Koppelaar, R., Jeanmart, H., 2017. Global available wind energy with physical and energy return on investment constraints. Applied Energy 209, 322-338. https://doi.org/10.1016/j.apenergy.2017.09.085

EABEV, 2008. Energy Consumption, CO2 Emissions and other considerations related to Battery Electric Vehicles. http://www.going-electric.org/.

EASAC, 2018. Negative emission technologies: What role in meeting Paris Agreement targets?

EC, 2010. Critical raw materials for the UE. Report of the Ad-hoc Working Group on defining critical raw materials. European Commission.

Edmonds, J., Pitcher, H., Sands, R., 2004. Second Generation Model 2004: An Overview. Joint Global Change Research Institute, Maryland.

Edmonds, J., Reilly, J.M., 1985. Global energy: Assessing the future. Oxford University Press, New York.

Edmonds, J., Smith, S., 2008. Integrated Assessment Modeling (Conference paper). Presented at the 13th Annual CCSM Workshop, 13th Annual CCSM Workshop, Breckenridge, Colorado, US.

EEA, 2018. Electric vehicles from life cycle and circular economy perspectives TERM 2018: Transport and Environment Reporting Mechanism (TERM) report (No. 13/2018), EEA Report. European Environment Agency, Luxembourg: Publications Office of the European Union.

EEA, 1997. Towards Sustainable Development for Local Authorities - Approaches, Experiences and Sources (Publication). European Environment Agency, Copenhagen, Denmark.

EEB, 2019. Decoupling debunked - Evidence and arguments against green growth as a sole strategy for sustainability. EEB - The European Environmental Bureau. URL https://eeb.org/library/decoupling-debunked/ (accessed 11.29.19).

EEGFTF, 2011. Future Transport Fuels Report. European Expert Group on Future Transport Fuels.

EERE, 2018. FOTW \#1040, July 30, 2018: Average Vehicle Occupancy Remains Unchanged From 2009 to 2017 [WWW Document]. Energy.gov. URL https://www.energy.gov/eere/vehicles/articles/fotw-1040-july-30-2018-averagevehicle-occupancy-remains-unchanged-2009-2017 (accessed 11.21.19).

Egede, P., 2017. Environmental Assessment of Lightweight Electric Vehicles, Sustainable Production, Life Cycle Engineering and Management. Springer International Publishing.

Eggler, L., 2020. D3.1 Review of information on IAMs. LOCOMOTION Project. European Commission.

Ehrlich, P.R., 1989. The limits to substitution: Meta-resource depletion and a new economicecological paradigm. Ecological Economics 1, 9-16. https://doi.org/10.1016/09218009(89)90021-9

Eker, S., Rovenskaya, E., Langan, S., Obersteiner, M., 2019. Model validation: A bibliometric analysis of the literature. Environmental Modelling \& Software 117, 43-54. https://doi.org/10.1016/j.envsoft.2019.03.009

Elshkaki, A., Graedel, T.E., 2013. Dynamic analysis of the global metals flows and stocks in electricity generation technologies. Journal of Cleaner Production 59, 260-273. https://doi.org/10.1016/j.jclepro.2013.07.003

Emmerling, J., Drouet, L., Reis, L., Bevione, M., Berger, L., Bosetti, V., Carrara, S., De Cian, E., de Maere d'Aertrycke, G., Longden, T., Malpede, M., Marangoni, G., Sferra, F., Tavoni, M., Witajewski-Baltvilks, J., Havlík, P., 2016. The WITCH 2016 Model - Documentation and Implementation of the Shared Socioeconomic Pathways (SSRN Scholarly Paper No. ID 2800970). Social Science Research Network, Rochester, NY.

Emsley, J., 2001. Nature's building blocks : an A-Z guide to the elements /. Oxford University Press.

Enríquez, J.M., Duce, C., Miguel, L.J., 2020. Repensar la sostenibilidad. UNED. 
European Comission, 2019. Opening Statement Ursula von der Leyen European Parliament [WWW Document]. European Commission - European Commission. URL https://ec.europa.eu/commission/presscorner/detail/en/speech_19_4230 (accessed 10.22.19).

Eurostat, 2018. Eurostat. Statistics Explained [WWW Document]. URL https://ec.europa.eu/eurostat/statistics-explained/index.php/Main_Page (accessed 11.21.19).

Evans, S., Housefather, Z., 2018. Q\&A: How "integrated assessment models" are used to study climate change. Carbon Brief. URL https://www.carbonbrief.org/qa-how-integratedassessment-models-are-used-to-study-climate-change (accessed 4.18.20).

EWG, 2013. Fossil and Nuclear Fuels - the Supply Outlook (No. 2013/03/18 LBST). Energy Watch Group.

EWG, 2008. Crude Oil - The Supply Outlook. Energy Watch Group / Ludwig-BoelkowFoundation.

EWG, 2007. Coal: Resources and Future Production (No. EWG-Paper No. 1/07).

EWG, 2006. Uranium Resources and Nuclear Energy (No. 1/2006), EWG-Series. Energy Watch Group.

Façanha, C., Blumberg, K., Miller, J., 2012. Global Transportation Energy and Climate Roadmap: The impact of transportation policies and their potential to reduce oil consumption and greenhouse gas emissions. Report, International Council on Clean Transportation, Washington http://www. theicct. org/sites/default/files/publications/ICCT\% 20Roadmap\% 20Energy\% 20Report. pdf.

Fan, X., Hu, E., Ji, X., Zhu, Y., Han, F., Hwang, S., Liu, J., Bak, S., Ma, Z., Gao, T., Liou, S.-C., Bai, J., Yang, X.-Q., Mo, Y., Xu, K., Su, D., Wang, C., 2018. High energy-density and reversibility of iron fluoride cathode enabled via an intercalation-extrusion reaction. Nature Communications 9, 1-12. https://doi.org/10.1038/s41467-018-04476-2

FAO, 2020. World Food and Agriculture 2020 Statistical Yearbook. https://doi.org/10.4060/cb1329en

FAOSTAT, 2019. Statistics Division of the FAO. Food and Agriculture Organization of the United Nations, Rome (Italy).

Fargione, J., Hill, J., Tilman, D., Polasky, S., Hawthorne, P., 2008. Land Clearing and the Biofuel Carbon Debt. Science 319, 1235-1238. https://doi.org/10.1126/science.1152747

Farley, J., Daly, H.E., 2003. Ecological Economics: Principles and Applications, 1 edition. ed. Island Press, Washington.

Featherston, C., Doolan, M., 2012. A Critical Review of the Criticisms of System Dynamics.

Fernández Durán, R., González Reyes, L., 2018. En la espiral de la energía, $2^{\text {a }}$ edición. ed. Libros en Acción, https://www.ecologistasenaccion.org/?p=29055.

Ferroni, F., Hopkirk, R.J., 2016. Energy Return on Energy Invested (ERoEI) for photovoltaic solar systems in regions of moderate insolation. Energy Policy 94, 336-344. https://doi.org/10.1016/j.enpol.2016.03.034

Fiddaman, T., Siegel, L.S., Sawin, E., Jones, A.P., Sterman, J., 2017. C-ROADS simulator reference guide (v78b) (No. v78b).

Fiddaman, T., Siegel, L.S., Sawin, E., Jones, A.P., Sterman, J., 2016. C-ROADS simulator reference guide (No. v74).

Fiddaman, T.S., 2002. Exploring policy options with a behavioral climate-economy model. Syst. Dyn. Rev. 18, 243-267. https://doi.org/10.1002/sdr.241

Fiddaman, T.S., 1997. Feedback complexity in integrated climate-economy models. Massachusetts Institute of Technology.

Field, C.B., Campbell, J.E., Lobell, D.B., 2008. Biomass energy: the scale of the potential resource. Trends in Ecology \& Evolution 23, 65-72.

https://doi.org/10.1016/j.tree.2007.12.001 
Filippini, M., Hunt, L.C., 2011. Energy Demand and Energy Efficiency in the OECD Countries: A Stochastic Demand Frontier Approach. The Energy Journal 32, 59-80.

Fischedick, M., Marzinkowski, J., Winzer, P., Weigel, M., 2014. Techno-economic evaluation of innovative steel production technologies. Journal of Cleaner Production, Special Volume: The sustainability agenda of the minerals and energy supply and demand network: an integrative analysis of ecological, ethical, economic, and technological dimensions 84, 563-580. https://doi.org/10.1016/j.jclepro.2014.05.063

Fisher, A.C., 1979. Measures of natural resource scarcity, in: Smith, V.K. (Ed.), Scarcity and Growth Reconsidered. pp. 249-275.

Fisher-Vanden, K., Schu, K., Sue Wing, I., Calvin, K., 2012. Decomposing the impact of alternative technology sets on future carbon emissions growth. Energy Economics, The Asia Modeling Exercise: Exploring the Role of Asia in Mitigating Climate Change 34, S359-S365. https://doi.org/10.1016/j.eneco.2012.07.021

Fleiter, T., Fehrenbach, D., Worrell, E., Eichhammer, W., 2012. Energy efficiency in the German pulp and paper industry - A model-based assessment of saving potentials. Energy 40, 84-99. https://doi.org/10.1016/j.energy.2012.02.025

Forrester, J., Senge, P., 1980. Tests for building confidence in system dynamics models.

Forrester, J.W., 1961. Industrial Dynamics. M.I.T. Press.

Fouquet, R., 2010. The slow search for solutions: Lessons from historical energy transitions by sector and service. Energy Policy 38, 6586-6596. https://doi.org/10.1016/j.enpol.2010.06.029

Freire-González, J., 2017. Evidence of direct and indirect rebound effect in households in EU-27 countries. Energy Policy 102, 270-276. https://doi.org/10.1016/j.enpol.2016.12.002

Frenzel, M., Ketris, M.P., Gutzmer, J., 2014. On the geological availability of germanium. Miner Deposita 49, 471-486. https://doi.org/10.1007/s00126-013-0506-z

Frenzel, M., Ketris, M.P., Seifert, T., Gutzmer, J., 2016. On the current and future availability of gallium. Resources Policy 47, 38-50. https://doi.org/10.1016/j.resourpol.2015.11.005

Fricko, O., Havlik, P., Rogelj, J., Klimont, Z., Gusti, M., Johnson, N., Kolp, P., Strubegger, M., Valin, H., Amann, M., Ermolieva, T., Forsell, N., Herrero, M., Heyes, C., Kindermann, G., Krey, V., McCollum, D.L., Obersteiner, M., Pachauri, S., Rao, S., Schmid, E., Schoepp, W., Riahi, K., 2017. The marker quantification of the Shared Socioeconomic Pathway 2: A middle-of-the-road scenario for the 21st century. Global Environmental Change 42, 251-267. https://doi.org/10.1016/j.gloenvcha.2016.06.004

Friedemann, A.J., 2016. When Trucks Stop Running, America Stops, in: Friedemann, A.J. (Ed.), When Trucks Stop Running: Energy and the Future of Transportation, SpringerBriefs in Energy. Springer International Publishing, Cham, pp. 1-3.

Fujimori, S., Dai, H., Masui, T., Matsuoka, Y., 2016. Global energy model hindcasting. Energy 114, 293-301. https://doi.org/10.1016/j.energy.2016.08.008

Fujimori, S., Hasegawa, T., Masui, T., 2017. AIM/CGE V2.0: Basic Feature of the Model, in: Fujimori, S., Kainuma, M., Masui, T. (Eds.), Post-2020 Climate Action: Global and Asian Perspectives. Springer Singapore, Singapore, pp. 305-328.

Fuss, S., Canadell, J.G., Peters, G.P., Tavoni, M., Andrew, R.M., Ciais, P., Jackson, R.B., Jones, C.D., Kraxner, F., Nakicenovic, N., Le Quéré, C., Raupach, M.R., Sharifi, A., Smith, P., Yamagata, Y., 2014. Betting on negative emissions. Nature Clim. Change 4, 850-853. https://doi.org/10.1038/nclimate2392

Gagnon, N., Hall, C.A.S., Brinker, L., 2009. A Preliminary Investigation of Energy Return on Energy Investment for Global Oil and Gas Production. Energies 2, 490-503. https://doi.org/10.3390/en20300490

Gambhir, A., Butnar, I., Li, P.-H., Smith, P., Strachan, N., 2019. A Review of Criticisms of Integrated Assessment Models and Proposed Approaches to Address These, through the Lens of BECCS. Energies 12, 1747. https://doi.org/10.3390/en12091747 
García-Olivares, A., Ballabrera-Poy, J., García-Ladona, E., Turiel, A., 2012. A global renewable mix with proven technologies and common materials. Energy Policy 41, 561-574. https://doi.org/10.1016/j.enpol.2011.11.018

García-Olivares, A., Solé, J., Osychenko, O., 2018. Transportation in a $100 \%$ renewable energy system. Energy Conversion and Management 158, 266-285. https://doi.org/10.1016/j.enconman.2017.12.053

Genty, A., Arto, I., Neuwahl, F., 2012. Final database of environmental satellite accounts: technical report on their compilation. WIOD Deliverable 4.6, Documentation, downloadable at http://www.wiod.org/publications/source_docs/Environmental_Sources.pdf.

Georgescu-Roegen, N., 1975. Dynamic models and economic growth. World Development 3, 765-783. https://doi.org/10.1016/0305-750X(75)90079-0

Georgescu-Roegen, N., 1971. The Entropy Law and the Economic Process, Reprint 2014 ed. edition. ed. Harvard University Press, Cambridge, Mass.

Gernaat, D.E.H.J., Bogaart, P.W., Vuuren, D.P. van, Biemans, H., Niessink, R., 2017. Highresolution assessment of global technical and economic hydropower potential. Nature Energy 2, 821-828. https://doi.org/10.1038/s41560-017-0006-y

Giampietro, M., Mayumi, K., 2009. The biofuel delusion: The fallacy of large scale agro-biofuels production. Routledge.

Girod, B., van Vuuren, D.P., de Vries, B., 2013. Influence of travel behavior on global CO2 emissions. Transportation Research Part A: Policy and Practice 50, 183-197. https://doi.org/10.1016/j.tra.2013.01.046

Gnann, T., Kühn, A., Plötz, P., Wietschel, M., 2017. How to decarbonise heavy road transport?

Gomiero, T., 2015. Are Biofuels an Effective and Viable Energy Strategy for Industrialized Societies? A Reasoned Overview of Potentials and Limits. Sustainability 7, 8491-8521. https://doi.org/10.3390/su7078491

Gotzens, F., Heinrichs, H., Hake, J.-F., Allelein, H.-J., 2018. The influence of continued reductions in renewable energy cost on the European electricity system. Energy Strategy Reviews 21, 71-81. https://doi.org/10.1016/j.esr.2018.04.007

Goudriaan, J., Ketner, P., 1984. A simulation study for the global carbon cycle, including man's impact on the biosphere. Climatic Change 6, 167-192. https://doi.org/10.1007/BF00144611

Graedel, T.E., Cao, J., 2010. Metal spectra as indicators of development. PNAS. https://doi.org/10.1073/pnas.1011019107

Graedel, T.E., Harper, E.M., Nassar, N.T., Nuss, P., Reck, B.K., 2015. Criticality of metals and metalloids. PNAS 112, 4257-4262. https://doi.org/10.1073/pnas.1500415112

Greene, D.L., 1999. An assessment of energy and environmental issues related to the use of gas-to-liquid fuels in transportation (No. ORNL/TM-1999/258). Oak Ridge National Lab., Oak Ridge, TN (US).

Grubler, A., Wilson, C., Bento, N., Boza-Kiss, B., Krey, V., McCollum, D.L., Rao, N.D., Riahi, K., Rogelj, J., De Stercke, S., Cullen, J., Frank, S., Fricko, O., Guo, F., Gidden, M., Havlík, P., Huppmann, D., Kiesewetter, G., Rafaj, P., Schoepp, W., Valin, H., 2018. A low energy demand scenario for meeting the $1.5^{\circ} \mathrm{C}$ target and sustainable development goals without negative emission technologies. Nature Energy 3, 515-527. https://doi.org/10.1038/s41560-018-0172-6

GWEC, 2017. Global Wind Report 2016. Global Wind Energy Council. http://gwec.net.

Haberl, H., Sprinz, D., Bonazountas, M., Cocco, P., Desaubies, Y., Henze, M., Hertel, O., Johnson, R.K., Kastrup, U., Laconte, P., Lange, E., Novak, P., Paavola, J., Reenberg, A., van den Hove, S., Vermeire, T., Wadhams, P., Searchinger, T., 2012. Correcting a fundamental error in greenhouse gas accounting related to bioenergy. Energy Policy 45, 18-23. https://doi.org/10.1016/j.enpol.2012.02.051 
Hall, C.A.S., 2017. Energy Return on Investment as Master Driver of Evolution, in: Energy Return on Investment. A Unifying Principle for Biology, Economics and Sustainability. pp. 59-72.

Hall, C.A.S., Balogh, S., Murphy, D.J.R., 2009. What is the Minimum EROI that a Sustainable Society Must Have? Energies 2, 25-47. https://doi.org/10.3390/en20100025

Hall, C.A.S., Klitgaard, K.A., 2012. Energy and the Wealth of Nations: Understanding the Biophysical Economy. Springer New York, New York, NY.

Hamilton, J.D., 2011. Historical Oil Shocks (Working Paper No. 16790). National Bureau of Economic Research.

Hamilton, S.H., Fu, B., Guillaume, J.H.A., Badham, J., Elsawah, S., Gober, P., Hunt, R.J., Iwanaga, T., Jakeman, A.J., Ames, D.P., Curtis, A., Hill, M.C., Pierce, S.A., Zare, F., 2019. A framework for characterising and evaluating the effectiveness of environmental modelling. Environmental Modelling \& Software 118, 83-98. https://doi.org/10.1016/j.envsoft.2019.04.008

Hammond, G., Jones, C., 2011. Inventory of Carbon \& Energy (ICE) Version 2.0. Sustainable Energy Research Team (SERT) Department of Mechanical Engineering University of Bath, UK.

Hannula, I., Reiner, D.M., 2019. Near-Term Potential of Biofuels, Electrofuels, and Battery Electric Vehicles in Decarbonizing Road Transport. Joule 3, 2390-2402. https://doi.org/10.1016/j.joule.2019.08.013

Hansen, J., Sato, M., Hearty, P., Ruedy, R., Kelley, M., Masson-Delmotte, V., Russell, G., Tselioudis, G., Cao, J., Rignot, E., Velicogna, I., Tormey, B., Donovan, B., Kandiano, E., von Schuckmann, K., Kharecha, P., Legrande, A.N., Bauer, M., Lo, K.-W., 2016. Ice melt, sea level rise and superstorms: evidence from paleoclimate data, climate modeling, and modern observations that $2{ }^{\circ} \mathrm{C}$ global warming could be dangerous. Atmos. Chem. Phys. 16, 3761-3812. https://doi.org/10.5194/acp-16-3761-2016

Hardt, L., O’Neill, D.W., 2017. Ecological Macroeconomic Models: Assessing Current Developments. Ecological Economics 134, 198-211. https://doi.org/10.1016/j.ecolecon.2016.12.027

Harmsen, J.H.M., Roes, A.L., Patel, M.K., 2013. The impact of copper scarcity on the efficiency of 2050 global renewable energy scenarios. Energy 50, 62-73. https://doi.org/10.1016/j.energy.2012.12.006

Harper, G., Sommerville, R., Kendrick, E., Driscoll, L., Slater, P., Stolkin, R., Walton, A., Christensen, P., Heidrich, O., Lambert, S., Abbott, A., Ryder, K., Gaines, L., Anderson, P., 2019. Recycling lithium-ion batteries from electric vehicles. Nature $575,75-86$. https://doi.org/10.1038/s41586-019-1682-5

Hejazi, M., Edmonds, J., Clarke, L., Kyle, P., Davies, E., Chaturvedi, V., Wise, M., Patel, P., Eom, J., Calvin, K., Moss, R., Kim, S., 2014. Long-term global water projections using six socioeconomic scenarios in an integrated assessment modeling framework. Technological Forecasting and Social Change 81, 205-226. https://doi.org/10.1016/j.techfore.2013.05.006

Hekkert, M.P., Hendriks, F.H.J.F., Faaij, A.P.C., Neelis, M.L., 2005. Natural gas as an alternative to crude oil in automotive fuel chains well-to-wheel analysis and transition strategy development. Energy Policy 33, 579-594. https://doi.org/10.1016/j.enpol.2003.08.018

Hernandez, R.R., Hoffacker, M.K., Murphy-Mariscal, M.L., Wu, G.C., Allen, M.F., 2015. Solar energy development impacts on land cover change and protected areas. PNAS 201517656. https://doi.org/10.1073/pnas.1517656112

Heun, M.K., Carbajales-Dale, M., Haney, B.R., 2015. Beyond GDP: National Accounting in the Age of Resource Depletion, Lecture Notes in Energy, Energy Analysis. Springer International Publishing.

Hickel, J., Kallis, G., 2019. Is Green Growth Possible? New Political Economy 0, 1-18. https://doi.org/10.1080/13563467.2019.1598964 
Hill, S.J., Lo, J., Vavreck, L., Zaller, J., 2013. How Quickly We Forget: The Duration of Persuasion Effects From Mass Communication. Political Communication 30, 521-547. https://doi.org/10.1080/10584609.2013.828143

Hirsch, R.L., 2008. Mitigation of maximum world oil production: Shortage scenarios. Energy Policy 36, 881-889. https://doi.org/10.1016/j.enpol.2007.11.009

Höök, M., Aleklett, K., 2010. A review on coal-to-liquid fuels and its coal consumption. International Journal of Energy Research 34, 848-864. https://doi.org/10.1002/er.1596

Höök, M., Tang, X., 2013. Depletion of fossil fuels and anthropogenic climate change-A review. Energy Policy 52, 797-809. https://doi.org/10.1016/j.enpol.2012.10.046

Höök, M., Zittel, W., Schindler, J., Aleklett, K., 2010. Global coal production outlooks based on a logistic model. Fuel 89, 3546-3558. https://doi.org/10.1016/j.fuel.2010.06.013

Hope, C., 2011. The Social Cost of CO2 from the PAGE09 Model. Cambridge Judge Business School Working Papers, Cambridge, UK.

Hopkinson, L., Cairns, S., Sloman, L., Newson, C., Hiblin, B., 2019. Radical Transport Policy TwoPagers | Transport for Quality of Life.

Hourcade, J.C., Jaccard, M., Bataille, C., Ghersi, F., 2006. Hybrid Modeling: New Answers to Old Challenges. The Energy Journal 2, 1-12.

House, K.Z., 2009. The limits of energy storage technology. Bulletin of the Atomic Scientists. URL https://thebulletin.org/2009/01/the-limits-of-energy-storage-technology/ (accessed 11.29.19).

Howarth, R.W., 2015. Methane emissions and climatic warming risk from hydraulic fracturing and shale gas development: implications for policy. EECT 3, 45-54. https://doi.org/10.2147/EECT.S61539

Howarth, R.W., Santoro, R., Ingraffea, A., 2011. Methane and the greenhouse-gas footprint of natural gas from shale formations. Climatic Change 106, 679-690. https://doi.org/10.1007/s10584-011-0061-5

Hubbert, M.K., 1956. Nuclear Energy and the Fossil Fuel, in: Drilling and Production Practice. American Petroleum Institute, San Antonio (Texas), US.

Hughes, B.B., Hossain, A., Irfan, M.T., 2004. The Structure of International Futures (IFs). WORKING PAPER 2004.07.19. Pardee Center for International Futures, Denver.

Hughes, J.D., 2013a. Drill Baby Drill: Can Unconventional Fuels Usher in a New Era of Energy Abundance?, 1st ed. CreateSpace Independent Publishing Platform.

Hughes, J.D., 2013b. Energy: A reality check on the shale revolution. Nature 494, 307-308. https://doi.org/10.1038/494307a

IAMC, 2020. Evaluation and diagnostics - iamconsortium. URL https://www.iamconsortium.org/scientific-working-groups/evaluation-anddiagnostics/ (accessed 11.20.20).

IEA, 2019a. Material efficiency in clean energy transitions. International Energy Agency.

IEA, 2019b. World Energy Model Documentation. International Energy Agency.

IEA, 2019c. IEA World Energy Statistics and Balances, World Energy Statistics and Balances (database). IEA/OECD, Paris (France).

IEA, 2019d. The Future of Rail Opportunities for energy and the environment. International Energy Agency.

IEA, 2018. World Energy Output 2018. IEA/OECD, Paris (France).

IEA, 2017a. The Future of Trucks Implications for energy and the environment. IEA/OECD, Paris. https://doi.org/10.1787/9789264279452-en.

IEA, 2017b. Energy Technology Perspectives 2017. IEA/OECD.

IEA, 2017c. World Energy Outlook 2017. OECD / IEA, Paris.

IEA, 2017d. Global EV Outlook 2017: Two million and counting. International Energy Agency, Paris. https://doi.org/10.1787/9789264278882-en.

IEA, 2016a. Energy Technology Perspectives 2016. IEA/OECD. 
IEA, 2016b. Global EV Outlook 2016: Beyond one million electric cars. IEA/OECD, Paris. https://doi.org/10.1787/9789264279469-en.

IEA, 2014. World Energy Outlook 2014. IEA/OECD, Paris (France).

IEA, 2013. Railway Handbook 2013 Energy Consumption and CO2 Emissions - Focus on Energy Mix. IEA/OECD.

IEA, 2012. World Energy Outlook 2012. IEA/OECD, Paris (France).

IEA, 2010a. World Energy Outlook 2010. IEA/OECD, Paris (France).

IEA, 2010b. The contribution of natural gas vehicles to sustainable transport. OECD Publishing.

IEA, 2009. Transport, Energy and CO2. Moving toward sustainability. IEA/OECD, Paris. https://doi.org/10.1787/9789264073173-en.

IEA, IRENA, 2017. IEA, and IRENA. "Perspectives for the Energy Transition. Investment Needs for a Low-Carbon Energy System." International Energy Agency and International Renewable Energy Agency.

IMAGE contributors, 2019. Welcome to IMAGE 3.0 Documentation - IMAGE [WWW Document]. URL

https://models.pbl.nl/image/index.php?title=Welcome_to_IMAGE_3.0_Documentatio n\&oldid=33240 (accessed 7.2.19).

Ioannou, P., Sun, J., 2013. Robust Adaptive Control. Courier Corporation.

IPCC, 2019. Climate Change and Land: an IPCC special report on climate change, desertification, land degradation, sustainable land management, food security, and greenhouse gas fluxes in terrestrial ecosystems [P.R. Shukla, J. Skea, E. Calvo Buendia, V. Masson-Delmotte, H.-O. Pörtner, D. C. Roberts, P. Zhai, R. Slade, S. Connors, R. van Diemen, M. Ferrat, E. Haughey, S. Luz, S. Neogi, M. Pathak, J. Petzold, J. Portugal Pereira, P. Vyas, E. Huntley, K. Kissick, M. Belkacemi, J. Malley, (eds.)]. In press.

IPCC, 2018. Global Warming of $1.5^{\circ} \mathrm{C}$ (IPCC Special Report). Intergovernmental Panel on Climate Change (IPCC). http://www.ipcc.ch/report/sr15/.

IPCC, 2014. Climate Change 2014: Mitigation of Climate Change. Cambridge University Press.

IPCC, 2012. Renewable Energy Sources and Climate Change Mitigation-Special Report of the Intergovernmental Panel on Climate Change. IPCC, New York.

IPCC, 2011. Special Report on Renewable Energy Sources and Climate Change Mitigation. Cambridge University Press, United Kingdom and New York (USA).

IPCC, 2007. Mitigation of Climate Change - Contribution of Working Group III. Fourth Assessment Report of the Intergovernmental Panel on Climate Change. Cambridge University Press.

IPCC, 2001. Climate Change 2001: Synthesis Report. A Contribution of Working Groups I, II, and III to the Third Assessment Report of the Integovernmental Panel on Climate Change [Watson, R.T. and the Core Writing Team (eds.)]. Cambridge University Press, Cambridge, United Kingdom, and New York, NY, USA.

IPCC, 1992. IPCC First Assessment Report Overview and Policymaker Summaries and 1992 IPCC Supplement.

IRENA db, 2019. IRENA Resource (Database). International Renewable Energy Agency, http://resourceirena.irena.org.

IRIZAR, 2015. i2e: $12 \mathrm{~m}$ urban bus with 100\% electric traction and climate control (PRODUCT/SERVICE). IRIZAR S. Coop.

Jacobson, M., A. Delucchi, M., Bazouin, G., F. Bauer, Z.A., C. Heavey, C., Fisher, E., B. Morris, S., Y. Piekutowski, D.J., A. Vencill, T., W. Yeskoo, T., 2015. 100\% clean and renewable wind, water, and sunlight (WWS) all-sector energy roadmaps for the 50 United States. Energy \& Environmental Science 8, 2093-2117. https://doi.org/10.1039/C5EE01283J

Jacobson, M.Z., Delucchi, M.A., 2011. Providing all global energy with wind, water, and solar power, Part I: Technologies, energy resources, quantities and areas of infrastructure, and materials. Energy Policy 39, 1154-1169.

https://doi.org/10.1016/j.enpol.2010.11.040 
James, D.E., Jansen, H.M.A., Opschoor, J.B., 1978. Economic Approaches to Environmental Problems. Elsevier North Holland, Amsterdam.

Janetos, A., Clarke, L., Collins, W., Ebi, K., Edmonds, J., Foster, I., Jacoby, H., Judd, K., Leung, L., Newell, R., others, 2009. Science challenges and future directions: climate change integrated assessment research. Dept. of Energy, Washington.

Kaack, L.H., Vaishnav, P., Morgan, M.G., Azevedo, I.L., Rai, S., 2018. Decarbonizing intraregional freight systems with a focus on modal shift. Environ. Res. Lett. 13, 83001. https://doi.org/10.1088/1748-9326/aad56c

Karkatsoulis, P., Siskos, P., Paroussos, L., Capros, P., 2017. Simulating deep CO2 emission reduction in transport in a general equilibrium framework: The GEM-E3T model. Transportation Research Part D: Transport and Environment 55, 343-358. https://doi.org/10.1016/j.trd.2016.11.026

Kartha, S., Dooley, K., 2016. The risks of relying on tomorrow" $s$ "negative emissions" to guide today" s mitigation action. Working Paper 2016-08, Stockholm Environment Institute. Retrieved 4 October, 2016 from https://www. sei-international. org/publications.

Kastner, T., Rivas, M.J.I., Koch, W., Nonhebel, S., 2012. Global changes in diets and the consequences for land requirements for food. PNAS 109, 6868-6872. https://doi.org/10.1073/pnas.1117054109

Kaya, A., Csala, D., Sgouridis, S., 2017. Constant elasticity of substitution functions for energy modeling in general equilibrium integrated assessment models: a critical review and recommendations. Climatic Change 145, 27-40. https://doi.org/10.1007/s10584-0172077-y

Kc, S., Lutz, W., 2017. The human core of the shared socioeconomic pathways: Population scenarios by age, sex and level of education for all countries to 2100 . Global Environmental Change 42, 181-192. https://doi.org/10.1016/j.gloenvcha.2014.06.004

Keith, D.W., DeCarolis, J.F., Denkenberger, D.C., Lenschow, D.H., Malyshev, S.L., Pacala, S., Rasch, P.J., 2004. The influence of large-scale wind power on global climate. PNAS 101, 16115-16120. https://doi.org/10.1073/pnas.0406930101

Kemfert, C., 2005. Induced technological change in a multi-regional, multi-sectoral, integrated assessment model (WIAGEM): Impact assessment of climate policy strategies. Ecological Economics, Technological Change and the Environment 54, 293-305. https://doi.org/10.1016/j.ecolecon.2004.12.031

Kerschner, C., Capellán-Pérez, I., 2017. Peak-Oil and Ecological Economics, in: Spash, C.L. (Ed.), Routdlege Handbook of Ecological Economics: Nature and Society. Abingdon, pp. 425435.

Kerschner, C., O'Neill, D.W., 2016. Economic Growth and Sustainability, in: Kopnina, H., Shoreman-Ouimet, E. (Eds.), Sustainability. Key Issues, Key Issues in Environment and Sustainability. Routledge, p. 392.

Kerschner, C., Wächter, P., Nierling, L., Ehlers, M.-H., 2018. Degrowth and Technology: Towards feasible, viable, appropriate and convivial imaginaries. Journal of Cleaner Production, Technology and Degrowth 197, 1619-1636. https://doi.org/10.1016/j.jclepro.2018.07.147

Kessides, I.N., Wade, D.C., 2011. Deriving an Improved Dynamic EROI to Provide Better Information for Energy Planners. Sustainability 3, 2339-2357. https://doi.org/10.3390/su3122339

Kim, H.C., Wallington, T.J., 2013. Life-Cycle Energy and Greenhouse Gas Emission Benefits of Lightweighting in Automobiles: Review and Harmonization. Environ. Sci. Technol. 47, 6089-6097. https://doi.org/10.1021/es3042115

King, C.W., 2020. An integrated biophysical and economic modeling framework for long-term sustainability analysis: the HARMONEY model. Ecological Economics 169, 106464. https://doi.org/10.1016/j.ecolecon.2019.106464 
King, C.W., 2016. Information Theory to Assess Relations Between Energy and Structure of the U.S. Economy Over Time. Biophys Econ Resour Qual 1, 10. https://doi.org/10.1007/s41247-016-0011-y

King, C.W., Maxwell, J.P., Donovan, A., 2015. Comparing World Economic and Net Energy Metrics, Part 1: Single Technology and Commodity Perspective. Energies 8, 1294912974. https://doi.org/10.3390/en81112346

Knutti, R., Rogelj, J., Sedláček, J., Fischer, E.M., 2016. A scientific critique of the two-degree climate change target. Nature Geoscience 9, 13-18. https://doi.org/10.1038/ngeo2595

Kousky, C., 2014. Informing climate adaptation: A review of the economic costs of natural disasters. Energy Economics 46, 576-592. https://doi.org/10.1016/j.eneco.2013.09.029

Krey, V., Havlik, P., Fricko, O., Zilliacus, J., Gidden, M., Strubegger, M., Kartasasmita, G., Ermolieva, T., Forsell, N., Gusti, M., Johnson, N., Kindermann, G., Kolp, P., McCollum, D.L., Pachauri, S., Rao, S., Rogelj, J., Valin, H., Obersteiner, M., Riahi, K., 2016. MESSAGE-GLOBIOM 1.0 Documentation. International Institute for Applied Systems Analysis (IIASA), Laxenburg, Austria.

Kriegler, E., O'Neill, B.C., Hallegatte, S., Kram, T., Lempert, R.J., Moss, R.H., Wilbanks, T., 2012. The need for and use of socio-economic scenarios for climate change analysis: A new approach based on shared socio-economic pathways. Global Environmental Change 22, 807-822. https://doi.org/10.1016/j.gloenvcha.2012.05.005

Kriegler, E., Riahi, K., Bauer, N., Schwanitz, V.J., Petermann, N., Bosetti, V., Marcucci, A., Otto, S., Paroussos, L., Rao, S., Arroyo Currás, T., Ashina, S., Bollen, J., Eom, J., Hamdi-Cherif, M., Longden, T., Kitous, A., Méjean, A., Sano, F., Schaeffer, M., Wada, K., Capros, P., P. van Vuuren, D., Edenhofer, O., 2015. Making or breaking climate targets: The AMPERE study on staged accession scenarios for climate policy. Technological Forecasting and Social Change 90, 24-44. https://doi.org/10.1016/j.techfore.2013.09.021

Kubiszewski, I., Costanza, R., Franco, C., Lawn, P., Talberth, J., Jackson, T., Aylmer, C., 2013. Beyond GDP: Measuring and achieving global genuine progress. Ecological Economics 93, 57-68. https://doi.org/10.1016/j.ecolecon.2013.04.019

Kyle, G.P., Luckow, P., Calvin, K.V., Emanuel, W.R., Nathan, M., Zhou, Y., 2011. GCAM 3.0 Agriculture and Land Use: Data Sources and Methods (No. PNNL-21025). Pacific Northwest National Lab. (PNNL), Richland, WA (United States), https://doi.org/10.2172/1036082.

Lacroix, K., 2018. Comparing the relative mitigation potential of individual pro-environmental behaviors. Journal of Cleaner Production 195, 1398-1407. https://doi.org/10.1016/j.jclepro.2018.05.068

Laherrère, J., 2018. Oil \& gas production forecasts (1900-2200) (Personal communication (April 2018)).

Laherrère, J., 2013. Oil \& gas production forecasts 1900-2100. Clarmix GEP/AFTP.

Laherrère, J., 2010. Peak Oil y Seguridad Energética. Presented at the Segundo Simposio ASPO Argentina Buenos Aires, Buenos Aires (Argentina).

Laherrère, J., 2006. Oil and gas, what future? Presented at the Groningen annual Energy Convention, Groningen, Netherlands.

Lamb, W.F., Steinberger, J.K., 2017. Human well-being and climate change mitigation. Wiley Interdisciplinary Reviews: Climate Change 8, e485. https://doi.org/10.1002/wcc.485

Lassaletta, L., Billen, G., Grizzetti, B., Garnier, J., Leach, A.M., Galloway, J.N., 2014. Food and feed trade as a driver in the global nitrogen cycle: 50 -year trends. Biogeochemistry 117. https://doi.org/10.1007/s10533-013-9923-4

Lavoie, M., 2014. Post-Keynesian Economics: New Foundations. Edward Elgar Publishing.

Lebedeva, N., Di Persio, F., Boon-Brett, L., 2016. Lithium ion battery value chain and related opportunities for Europe. Joint Research Centre, https://doi.org/10.2760/6060. 
Lejour, A., Veenendaal, P., Verweij, G., Van Leeuwen, N., 2006. WorldScan: a Model for International Economic Policy Analysis. CPB Netherlands Bureau for Economic Policy Analysis, The Hague.

Lenton, T.M., Ciscar, J.-C., 2013. Integrating tipping points into climate impact assessments. Climatic Change 117, 585-597. https://doi.org/10.1007/s10584-012-0572-8

Lenton, T.M., Held, H., Kriegler, E., Hall, J.W., Lucht, W., Rahmstorf, S., Schellnhuber, H.J., 2008. Tipping elements in the Earth's climate system. PNAS 105, 1786-1793. https://doi.org/10.1073/pnas.0705414105

Lenzen, M., 2010. Current State of Development of Electricity-Generating Technologies: A Literature Review. Energies 3, 462-591. https://doi.org/10.3390/en3030462

Lenzi, D., Lamb, W.F., Hilaire, J., Kowarsch, M., Minx, J.C., 2018. Don't deploy negative emissions technologies without ethical analysis. Nature 561, 303-305. https://doi.org/10.1038/d41586-018-06695-5

Leontief, W. (Ed.), 1986. Input-Output Economics, Second Edition. ed. Oxford University Press, Oxford, New York.

Leontief, W., 1970. Environmental Repercussions and the Economic Structure: An InputOutput Approach. The Review of Economics and Statistics 52, 262-271. https://doi.org/10.2307/1926294

Levin, S.A., Carpenter, S.R., Godfray, H.C.J., Kinzig, A.P., Loreau, M., Losos, J.B., Walker, B., Wilcove, D.S., 2009. The Princeton guide to ecology. Princeton University Press, Princeton, N.J. (USA).

Li, B., Li, J., Yuan, C., 2013. Life Cycle Assessment of Lithium Ion Batteries with Silicon Nanowire Anode for Electric Vehicles. Presented at the 2013 IEEE International Symposium on Sustainable Systems \& Technology (ISSST 2013). https://doi.org/10.6084/m9.figshare.805147

Lightfoot, H.D., Green, C., 2002. Energy intensity decline implications for stabilization of atmospheric CO2 content (No. Report No. 2001-7, October 2001). McGill Centre for Climate and Global Change Research (C2GCR).

Limpens, G., Jeanmart, H., 2018. Electricity storage needs for the energy transition: An EROI based analysis illustrated by the case of Belgium. Energy 152, 960-973. https://doi.org/10.1016/j.energy.2018.03.180

Lontzek, T.S., Cai, Y., Judd, K.L., Lenton, T.M., 2015. Stochastic integrated assessment of climate tipping points indicates the need for strict climate policy. Nature Climate Change 5, 441-444. https://doi.org/10.1038/nclimate2570

Loulou, R., Goldstein, G., Noble, K., 2004. Documentation for the MARKAL Family of Models.

Lucas, A., Alexandra Silva, C., Costa Neto, R., 2012. Life cycle analysis of energy supply infrastructure for conventional and electric vehicles. Energy Policy, Modeling Transport (Energy) Demand and Policies 41, 537-547. https://doi.org/10.1016/j.enpol.2011.11.015

Luderer, G., Leimbach, M., Bauer, N., Kriegler, E., Baumstark, L., Bertram, C., Giannousakis, A., Hilaire, J., Klein, D., Levesque, A., Mouratiadou, I., Pehl, M., Pietzcker, R., Piontek, F., Roming, N., Schultes, A., Schwanitz, V.J., Strefler, J., 2015. Description of the REMIND Model (Version 1.6) (SSRN Scholarly Paper No. ID 2697070). Social Science Research Network, Rochester, NY.

Luderer, G., Vrontisi, Z., Bertram, C., Edelenbosch, O.Y., Pietzcker, R.C., Rogelj, J., De Boer, H.S., Drouet, L., Emmerling, J., Fricko, O., Fujimori, S., Havlík, P., Iyer, G., Keramidas, K., Kitous, A., Pehl, M., Krey, V., Riahi, K., Saveyn, B., Tavoni, M., Van Vuuren, D.P., Kriegler, E., 2018. Residual fossil CO 2 emissions in $1.5-2{ }^{\circ} \mathrm{C}$ pathways. Nature Climate Change 8, 626-633. https://doi.org/10.1038/s41558-018-0198-6

Lund, J.W., Boyd, T.L., 2015. Direct Utilization of Geothermal Energy 2015 Worldwide Review, in: Proceedings World Geothermal Congress 2015. Presented at the World Geothermal Congress 2015, Melbourne, Australia, p. 31. 
Maggio, G., Cacciola, G., 2012. When will oil, natural gas, and coal peak? Fuel 98, 111-123. https://doi.org/10.1016/j.fuel.2012.03.021

Malins, C., 2019. Destination Deforestation - Aviation biofuels, vegetable oil and land use change. Rainforest Foundation Norway.

MAN Truck \& Bus, 2020. MAN eTruck | electromobility in distribution transport | MAN Truck Germany [WWW Document]. URL https://www.truck.man.eu/de/en/man-etruck.html (accessed 3.12.20).

Manne, A.S., Richels, R.G., 2005. Merge: An Integrated Assessment Model for Global Climate Change, in: Loulou, R., Waaub, J.-P., Zaccour, G. (Eds.), Energy and Environment. Springer US, Boston, MA, pp. 175-189.

Marcucci, A., Turton, H., 2012. THE MERGE-ETL MODEL: MODEL DOCUMENTATION. Energy Economics Group Laboratory for Energy Analysis. The Energy Departments. Paul Scherrer Institute, Switzerland.

Masnadi, M.S., Brandt, A.R., 2017. Energetic productivity dynamics of global super-giant oilfields. Energy Environ. Sci. 10, 1493-1504. https://doi.org/10.1039/C7EE01031A

McCollum, D., Bauer, N., Calvin, K., Kitous, A., Riahi, K., 2014. Fossil resource and energy security dynamics in conventional and carbon-constrained worlds. Climatic Change 123, 413-426. https://doi.org/10.1007/s10584-013-0939-5

McCollum, D.L., Wilson, C., Bevione, M., Carrara, S., Edelenbosch, O.Y., Emmerling, J., Guivarch, C., Karkatsoulis, P., Keppo, I., Krey, V., Lin, Z., Broin, E.Ó., Paroussos, L., Pettifor, H., Ramea, K., Riahi, K., Sano, F., Rodriguez, B.S., van Vuuren, D.P., 2018a. Interaction of consumer preferences and climate policies in the global transition to low-carbon vehicles. Nature Energy 3, 664-673. https://doi.org/10.1038/s41560-0180195-z

McCollum, D.L., Wilson, C., Pettifor, H., Ramea, K., Krey, V., Riahi, K., Bertram, C., Lin, Z., Edelenbosch, O.Y., Fujisawa, S., 2017. Improving the behavioral realism of global integrated assessment models: An application to consumers' vehicle choices. Transportation Research Part D: Transport and Environment 55, 322-342. https://doi.org/10.1016/j.trd.2016.04.003

McCollum, D.L., Zhou, W., Bertram, C., de Boer, H.-S., Bosetti, V., Busch, S., Després, J., Drouet, L., Emmerling, J., Fay, M., Fricko, O., Fujimori, S., Gidden, M., Harmsen, M., Huppmann, D., Iyer, G., Krey, V., Kriegler, E., Nicolas, C., Pachauri, S., Parkinson, S., PobleteCazenave, M., Rafaj, P., Rao, N., Rozenberg, J., Schmitz, A., Schoepp, W., van Vuuren, D., Riahi, K., 2018b. Energy investment needs for fulfilling the Paris Agreement and achieving the Sustainable Development Goals. Nature Energy 3, 589-599. https://doi.org/10.1038/s41560-018-0179-z

McGlade, C.E., 2012. A review of the uncertainties in estimates of global oil resources. Energy, Asia-Pacific Forum on Renewable Energy 2011 47, 262-270. https://doi.org/10.1016/j.energy.2012.07.048

MEA, 2005. Millennium Ecosystem Assessment. Ecosystems and Human Well-being: Scenarios, Global Assessment Reports. Island Press, Washington DC (USA).

Meadows, D.H., Meadows, D.L., Randers, J., Behrens, W.W., 1972. The limits to growth. Universe Books, New York.

Meadows, D.H., Randers, J., Meadows, D.L., 2004. The limits to growth: the 30-year update. Chelsea Green Publishing Company, White River Junction, Vt.

Mediavilla, M., de Castro, C., Capellán, I., Javier Miguel, L., Arto, I., Frechoso, F., 2013. The transition towards renewable energies: Physical limits and temporal conditions. Energy Policy, Special Section: Transition Pathways to a Low Carbon Economy 52, 297-311. https://doi.org/10.1016/j.enpol.2012.09.033

Meinshausen, M., Raper, S., Wigley, T., 2011. Emulating coupled atmosphere-ocean and carbon cycle models with a simpler model, MAGICC6 - Part 1: Model description and 
calibration. Atmos. Chem. Phys. 11, 1417-1456. https://doi.org/10.5194/acp-11-14172011

Melin, H.E., 2019. State-of-the-art in reuse and recycling of lithium-ion batteries - A research review. Circular Energy Storage.

Messner, S., Strubegger, M., 1995. User's Guide for MESSAGE III. IIASA Working Paper. (No. WP-95-069). IIASA, Laxenburg, Austria.

Millennium Institute, 2017. iSDG Documentation [WWW Document]. Millennium Institute. URL https://www.millennium-institute.org/documentation (accessed 9.7.20).

Miller, L.M., Gans, F., Kleidon, A., 2011. Estimating maximum global land surface wind power extractability and associated climatic consequences. Earth System Dynamics 2, 1-12. https://doi.org/10.5194/esd-2-1-2011

Miller, L.M., Keith, D.W., 2018. Climatic Impacts of Wind Power. Joule 2, 2618-2632. https://doi.org/10.1016/j.joule.2018.09.009

Miller, L.M., Kleidon, A., 2016. Wind speed reductions by large-scale wind turbine deployments lower turbine efficiencies and set low generation limits. PNAS 201602253. https://doi.org/10.1073/pnas.1602253113

Miller, R.E., Blair, P.D., 2009. Input-Analysis. Foundations and Extensions. Cambridge University Press, Cambridge, UK.

Miller, R.G., Sorrell, S.R., 2014. The future of oil supply. Philosophical Transactions of the Royal Society of London A: Mathematical, Physical and Engineering Sciences 372, 20130179. https://doi.org/10.1098/rsta.2013.0179

Mohr, S.H., 2012. Fossil fuel future production, world and Australia focus. Presented the Australian Frontiers of Science 2012: Science for a green economy, Sydney, 2-4 December 2012.

Mohr, S.H., Evans, G.M., 2011. Long term forecasting of natural gas production. Energy Policy 39, 5550-5560. https://doi.org/10.1016/j.enpol.2011.04.066

Mohr, S.H., Evans, G.M., 2009. Forecasting coal production until 2100. Fuel 88, 2059-2067. https://doi.org/10.1016/j.fuel.2009.01.032

Mohr, S.H., Wang, J., Ellem, G., Ward, J., Giurco, D., 2015. Projection of world fossil fuels by country. Fuel 141, 120-135. https://doi.org/10.1016/j.fuel.2014.10.030

Moriarty, P., Honnery, D., 2016. Can renewable energy power the future? Energy Policy 93, 37. https://doi.org/10.1016/j.enpol.2016.02.051

Moriarty, P., Honnery, D., 2013. Greening passenger transport: a review. JOURNAL OF CLEANER PRODUCTION 54, 14-22. https://doi.org/10.1016/J.JCLEPRO.2013.04.008

Moriarty, P., Honnery, D., 2012. What is the global potential for renewable energy? Renewable and Sustainable Energy Reviews 16, 244-252. https://doi.org/10.1016/j.rser.2011.07.151

Moriarty, P., Honnery, D., 2008. Low-mobility: The future of transport. Futures 40, 865-872. https://doi.org/10.1016/j.futures.2008.07.021

Mottschall, M., Kasten, P., Rodríguez, F., 2020. Decarbonization of on-road freight transport and the role of LNG from a German perspective. International Council on Clean Transportation, Berlin.

Moultak, M., Lutsey, N., Hall, D., 2017. TRANSITIONING TO ZERO-EMISSION HEAVY-DUTY FREIGHT VEHICLES. International Council on Clean Transportation.

Moyer, E.J., Woolley, M.D., Matteson, N.J., Glotter, M.J., Weisbach, D.A., 2014. Climate impacts on economic growth as drivers of uncertainty in the social cost of carbon. The Journal of Legal Studies 43, 401-425.

Mudd, G.M., 2010. The Environmental sustainability of mining in Australia: key mega-trends and looming constraints. Resources Policy 35, 98-115. https://doi.org/10.1016/j.resourpol.2009.12.001

Mudd, G.M., Jowitt, S.M., Werner, T.T., 2017. The world's by-product and critical metal resources part I: Uncertainties, current reporting practices, implications and grounds 
for optimism. Ore Geology Reviews 86, 924-938.

https://doi.org/10.1016/j.oregeorev.2016.05.001

Murphy, D.J., Carbajales-Dale, M., Moeller, D., 2016. Comparing Apples to Apples: Why the Net Energy Analysis Community Needs to Adopt the Life-Cycle Analysis Framework. Energies 9, 917. https://doi.org/10.3390/en9110917

Murphy, D.J., Hall, C.A.S., 2011. Energy return on investment, peak oil, and the end of economic growth. Annals of the New York Academy of Sciences 1219, 52-72. https://doi.org/10.1111/j.1749-6632.2010.05940.x

Nakicenovic, N., Alcamo, J., Grubler, A., Riahi, K., Roehrl, R.A., Rogner, H.-H., Victor, N., 2000. Special Report on Emissions Scenarios (SRES), A Special Report of Working Group III of the Intergovernmental Panel on Climate Change. Cambridge University Press, Cambridge.

NAS, 2017. Valuing Climate Damages: Updating Estimation of the Social Cost of Carbon Dioxide. National Academies Press.

NASA Global Climate Change, 2020. Sea Level | NASA Global Climate Change [WWW Document]. Climate Change: Vital Signs of the Planet. URL https://climate.nasa.gov/vital-signs/sea-level (accessed 1.8.21).

Natalini, D., Buchmann, K., Spannenkrebs, R., Nitschke, S., Jones, A., Perissi, I., Falsini, S., Bardi, U., Nikolaev, A., Baumann, M., Egler, L., Angela, H., Ploier, C., Capellán-Pérez, I., Miguel González, L.J., Henke, H.T.J., 2018. D5.1 Models' cross-comparison and qualitative evaluation (Deliverable MEDEAS project (Private) No. D5.1). MEDEAS project, Barcelona, Spain.

NCC, 2015. IAM helpful or not? Nature Climate Change 5, 81-81. https://doi.org/10.1038/nclimate2526

Neumayer, E., 2000. Scarce or Abundant? The Economics of Natural Resource Availability. Journal of Economic Surveys 14, 307-335. https://doi.org/10.1111/1467-6419.00112

Neumeyer, C., Goldston, R., 2016. Dynamic EROI Assessment of the IPCC 21st Century Electricity Production Scenario. Sustainability 8, 421. https://doi.org/10.3390/su8050421

NGV Global, 2019. Current Natural Gas Vehicle Statistics. Natural Gas Vehicle Knowledge Base, http://www.iangv.org/current-ngv-stats/.

Nieto, J., Carpintero, Ó., Miguel, L.J., de Blas, I., 2019. Macroeconomic modelling under energy constraints: Global low carbon transition scenarios. Energy Policy 111090. https://doi.org/10.1016/j.enpol.2019.111090

Nikas, A., Hawkes, A., Gambhir, A., Giarola, S., Bosello, F., Campagnolo, L., Moreno, J., van de Ven, D.-J., González-Eguino, M., Kolpakov, A., Shirov, A., Gargiulo, A.C., de Miglio, R., Vielle, M., Koasidis, K., Anger-Kraavi, A., 2019. D7.1 Documentation of Global IAMs. PARIS REINFORCE project. European Commission.

Nordhaus, W.D., 1992. The "Dice" Model: Background and Structure Of a Dynamic Integrated Climate-Economy Model of the Economics of Global Warning (Cowles Foundation Discussion Papers 1009). Cowles Foundation for Research in Economics, Yale Universisty, New Haven.

Nordhaus, W.D., 1979. The efficient use of energy resources, Cowles Foundation for Research in Economics, Yale Universisty. ed. Yale University Press, New Haven.

Nordhaus, W.D., Yang, Z., 1996. A Regional Dynamic General-Equilibrium Model of Alternative Climate-Change Strategies. The American Economic Review 86, 741-765.

Norgaard, R.B., 1990. Economic indicators of resource scarcity: A critical essay. Journal of Environmental Economics and Management 19, 19-25. https://doi.org/10.1016/00950696(90)90057-6

NREL, 2012. Renewable Electricity Futures Study (Entire Report) (4 vols. No. NREL/TP-6A2052409). National Renewable Energy Laboratory, Golden, CO, USA. 
OECD, 2019. Global Material Resources Outlook to 2060: Economic Drivers and Environmental Consequences. OECD Publishing, Paris (France).

Oeschger, H., Siegenthaler, U., Schotterer, U., Gugelmann, A., 1975. A box diffusion model to study the carbon dioxide exchange in nature. Tellus 27, 168-192. https://doi.org/10.3402/tellusa.v27i2.9900

OICA, 2019. World vehicles in use 2005-2015. International Organization of Motor Vehicle Manufacturers, http://www.oica.net/category/vehicles-in-use/.

O'Neill, B.C., Kriegler, E., Ebi, K.L., Kemp-Benedict, E., Riahi, K., Rothman, D.S., van Ruijven, B.J., van Vuuren, D.P., Birkmann, J., Kok, K., Levy, M., Solecki, W., 2017. The roads ahead: Narratives for shared socioeconomic pathways describing world futures in the 21st century. Global Environmental Change 42, 169-180. https://doi.org/10.1016/j.gloenvcha.2015.01.004

O’Neill, B.C., Kriegler, E., Riahi, K., Ebi, K.L., Hallegatte, S., Carter, T.R., Mathur, R., van Vuuren, D.P., 2014. A new scenario framework for climate change research: the concept of shared socioeconomic pathways. Climatic Change 122, 387-400. https://doi.org/10.1007/s10584-013-0905-2

Oreskes, N., 1998. Evaluation (not validation) of quantitative models. Environ Health Perspect 106, 1453-1460. https://doi.org/10.1289/ehp.98106s61453

Oreskes, N., Shrader-Frechette, K., Belitz, K., 1994. Verification, Validation, and Confirmation of Numerical Models in the Earth Sciences. Science 263, 641-646. https://doi.org/10.1126/science.263.5147.641

Overmars, K.P., Stehfest, E., Ros, J.P.M., Prins, A.G., 2011. Indirect land use change emissions related to EU biofuel consumption: an analysis based on historical data. Environmental Science \& Policy 14, 248-257. https://doi.org/10.1016/j.envsci.2010.12.012

Paleologu, C., Benesty, J., Ciochina, S., 2008. A Robust Variable Forgetting Factor Recursive Least-Squares Algorithm for System Identification. IEEE Signal Processing Letters 15, 597-600. https://doi.org/10.1109/LSP.2008.2001559

Palmer, G., 2018. A Biophysical Perspective of IPCC Integrated Energy Modelling. Energies 11, 839. https://doi.org/10.3390/en11040839

Papong, S., Chom-In, T., Noksa-nga, S., Malakul, P., 2010. Life cycle energy efficiency and potentials of biodiesel production from palm oil in Thailand. Energy Policy 38, 226233. https://doi.org/10.1016/j.enpol.2009.09.009

Pastor, A.V., Vieira, D.C.S., Soudijn, F.H., Edelenbosch, O.Y., 2020. How uncertainties are tackled in multi-disciplinary science? A review of integrated assessments under global change. CATENA 186, 104305. https://doi.org/10.1016/j.catena.2019.104305

Patzek, T.W., 2010. A Probabilistic Analysis of the Switchgrass Ethanol Cycle. Sustainability 2, 3158-3194. https://doi.org/10.3390/su2103158

Patzek, T.W., Croft, G.D., 2010. A global coal production forecast with multi-Hubbert cycle analysis. Energy 35, 3109-3122. https://doi.org/10.1016/j.energy.2010.02.009

Pezzey, J.C.V., Burke, P.J., 2014. Towards a more inclusive and precautionary indicator of global sustainability. Ecological Economics 106, 141-154. https://doi.org/10.1016/j.ecolecon.2014.07.008

Pfenninger, S., 2017. Energy scientists must show their workings. Nature News 542, 393. https://doi.org/10.1038/542393a

Pielke, R., Wigley, T., Green, C., 2008. Dangerous assumptions. Nature 452, 531-532. https://doi.org/10.1038/452531a

Pimentel, D., Marklein, A., Toth, M.A., Karpoff, M.N., Paul, G.S., McCormack, R., Kyriazis, J., Krueger, T., 2009. Food Versus Biofuels: Environmental and Economic Costs. Hum Ecol 37, 1-12. https://doi.org/10.1007/s10745-009-9215-8

Pimentel, D., Patzek, T.W., 2005. Ethanol Production Using Corn, Switchgrass, and Wood; Biodiesel Production Using Soybean and Sunflower. Nat Resour Res 14, 65-76. https://doi.org/10.1007/s11053-005-4679-8 
Pindyck, R.S., 2017. The Use and Misuse of Models for Climate Policy. Rev Environ Econ Policy 11, 100-114. https://doi.org/10.1093/reep/rew012

Pollitt, H., 2019. E3ME-Technical-Manual-v6.1. Cambridge Econometrics, Cambridge.

Prieto, P.A., Hall, C.A.S., 2013. Spain's Photovoltaic Revolution: The Energy Return on Investment, 2013th ed. Springer.

Prior, T., Giurco, D., Mudd, G., Mason, L., Behrisch, J., 2012. Resource depletion, peak minerals and the implications for sustainable resource management. Global Environmental Change, Global transformations, social metabolism and the dynamics of socioenvironmental conflicts 22, 577-587. https://doi.org/10.1016/j.gloenvcha.2011.08.009

Proskuryakova, L., Kovalev, A., 2015. Measuring energy efficiency: Is energy intensity a good evidence base? Applied Energy 138, 450-459. https://doi.org/10.1016/j.apenergy.2014.10.060

Qudrat-Ullah, H., 2012. On the validation of system dynamics type simulation models. Telecommun Syst 51, 159-166. https://doi.org/10.1007/s11235-011-9425-4

Radzicki, M., 2009. System Dynamics and Its Contribution to Economics and Economic Modeling. Encyclopedia of Complexity and Systems Science. https://doi.org/10.1007/978-0-387-30440-3_539

Raugei, M., Leccisi, E., 2016. A comprehensive assessment of the energy performance of the full range of electricity generation technologies deployed in the United Kingdom. Energy Policy 90, 46-59. https://doi.org/10.1016/j.enpol.2015.12.011

Raugei, M., Sgouridis, S., Murphy, D., Fthenakis, V., Frischknecht, R., Breyer, C., Bardi, U., Barnhart, C., Buckley, A., Carbajales-Dale, M., Csala, D., de Wild-Scholten, M., Heath, G., Jæger-Waldau, A., Jones, C., Keller, A., Leccisi, E., Mancarella, P., Pearsall, N., Siegel, A., Sinke, W., Stolz, P., 2017. Energy Return on Energy Invested (ERoEI) for photovoltaic solar systems in regions of moderate insolation: A comprehensive response. Energy Policy 102, 377-384. https://doi.org/10.1016/j.enpol.2016.12.042

Raworth, K., 2017. Doughnut Economics: Seven ways to think like a 21st-century economist. Random House.

RCP db, 2009. RCP Database (version 2.0). Available at: https://tntcat.iiasa.ac.at/RcpDb/dsd?Action=htmlpage \&page=welcome.

Reiner, D.M., 2016. Learning through a portfolio of carbon capture and storage demonstration projects. Nature Energy 1, 15011. https://doi.org/10.1038/nenergy.2015.11

Reynolds, D.B., 1999. The mineral economy: how prices and costs can falsely signal decreasing scarcity. Ecological Economics 31, 155-166. https://doi.org/10.1016/S09218009(99)00098-1

Riahi, K., Dentener, F., Gielen, D., Grubler, A., Jewell, J., Klimont, Z., Krey, V., McCollum, D.L., Pachauri, S., Rao, S., van Ruijven, B., van Vuuren, D.P., Wilson, C., 2012. Chapter 17: Energy pathways for sustainable development, in: Team, G.W. (Ed.), Global Energy Assessment: Toward a Sustainable Future. Cambridge University Press and IIASA, pp.1203-1306 (October 2012).

Riahi, K., van Vuuren, D.P., Kriegler, E., Edmonds, J., O’Neill, B.C., Fujimori, S., Bauer, N., Calvin, K., Dellink, R., Fricko, O., Lutz, W., Popp, A., Cuaresma, J.C., Kc, S., Leimbach, M., Jiang, L., Kram, T., Rao, S., Emmerling, J., Ebi, K., Hasegawa, T., Havlik, P., Humpenöder, F., Da Silva, L.A., Smith, S., Stehfest, E., Bosetti, V., Eom, J., Gernaat, D., Masui, T., Rogelj, J., Strefler, J., Drouet, L., Krey, V., Luderer, G., Harmsen, M., Takahashi, K., Baumstark, L., Doelman, J.C., Kainuma, M., Klimont, Z., Marangoni, G., Lotze-Campen, H., Obersteiner, M., Tabeau, A., Tavoni, M., 2017. The Shared Socioeconomic Pathways and their energy, land use, and greenhouse gas emissions implications: An overview. Global Environmental Change 42, 153-168. https://doi.org/10.1016/j.gloenvcha.2016.05.009

Ripple, W.J., Wolf, C., Newsome, T.M., Galetti, M., Alamgir, M., Crist, E., Mahmoud, M.I., Laurance, W.F., 15, 364 Scientist Signatories from 184 Countries, 2017. World 
Scientists' Warning to Humanity: A Second Notice. BioScience 67, 1026-1028. https://doi.org/10.1093/biosci/bix125

Risbey, J., Kandlikar, M., Patwardhan, A., 1996. Assessing integrated assessments. Climatic Change 34, 369-395. https://doi.org/10.1007/BF00139298

Ritchie, J., Dowlatabadi, H., 2017. The 1000 GtC coal question: Are cases of vastly expanded future coal combustion still plausible? Energy Economics 65, 16-31. https://doi.org/10.1016/j.eneco.2017.04.015

RITE, 2008. RITE Global model for Energy and Climate Change Assessment: DNE21+ [WWW Document]. URL https://www.rite.or.jp/system/en/global-warmingouyou/modeltodata/overviewdne21/ (accessed 9.17.20).

Robelius, F., 2007. Giant Oil Fields - The Highway to Oil: Giant Oil Fields and their Importance for Future Oil Production (dissertation). Uppsala University.

Rockström, J., Steffen, W., Noone, K., Persson, Å., Chapin, F.S., Lambin, E.F., Lenton, T.M., Scheffer, M., Folke, C., Schellnhuber, H.J., Nykvist, B., de Wit, C.A., Hughes, T., van der Leeuw, S., Rodhe, H., Sörlin, S., Snyder, P.K., Costanza, R., Svedin, U., Falkenmark, M., Karlberg, L., Corell, R.W., Fabry, V.J., Hansen, J., Walker, B., Liverman, D., Richardson, K., Crutzen, P., Foley, J.A., 2009. A safe operating space for humanity. Nature 461, 472-475. https://doi.org/10.1038/461472a

Roelfsema, M., van Soest, H.L., Hamsen, M., den Elzen, M., Höhne, N., lacubuta, G., Krey, V., Kriegler, E., Luderer, G., Riahi, K., Ueckerdt, F., Després, J., Drouet, L., Emmerling, J., Frank, S., Fricko, O., Gidden, M., Humpenöder, F., Huppmann, D., Fujimori, S., Fragkiadakis, K., Gi, K., Keramidas, K., Köberle, A.C., Reis, L.A., Rochedo, P., Schaeffer, R., Oshiro, K., Vrontisi, Z., Chen, W., Iyer, G.C., Edmonds, J., Kannavou, M., Jiang, K., Mathur, R., Safonov, G., Vishwanathan, S.S., 2020. Taking stock of national climate policies to evaluate implementation of the Paris Agreement. Nature Communications 11, e2096. https://doi.org/10.1038/s41467-020-15414-6

Rogelj, J., Popp, A., Calvin, K.V., Luderer, G., Emmerling, J., Gernaat, D., Fujimori, S., Strefler, J., Hasegawa, T., Marangoni, G., Krey, V., Kriegler, E., Riahi, K., van Vuuren, D.P.,

Doelman, J., Drouet, L., Edmonds, J., Fricko, O., Harmsen, M., Havlík, P., Humpenöder, F., Stehfest, E., Tavoni, M., 2018. Scenarios towards limiting global mean temperature increase below $1.5^{\circ} \mathrm{C}$. Nature Climate Change 8, 325-332. https://doi.org/10.1038/s41558-018-0091-3

Rogner, H.-H., Aguilera, R.F., Bertani, R., Bhattacharya, S.C., Dusseault, M.B., Gagnon, L., Haberl, H., Hoogwijk, M., Johnson, A., Rogner, M.L., Wagner, H., Yakushev, V., 2012. Chapter 7 - Energy Resources and Potentials, in: Global Energy Assessment - Toward a Sustainable Future. Cambridge University Press, Cambridge, UK and New York, NY, USA and the International Institute for Applied Systems Analysis, Laxenburg, Austria, pp. 423-512.

Rosen, R.A., 2015. Critical review of: "Making or breaking climate targets - the AMPERE study on staged accession scenarios for climate policy." Technological Forecasting and Social Change 96, 322-326. https://doi.org/10.1016/j.techfore.2015.01.019

Rotmans, J., 1990. Image: An Integrated Model to Assess the Greenhouse Effect. Springer.

Rydzak, F., Obersteiner, M., Kraxner, F., Fritz, S., McCallum, I., 2013. FeliX3 - Impact Assessment Model. Model Report and Technical Documentation. IIASA, Laxenburg, Austria.

Rye, C.D., Jackson, T., 2018. A review of EROEI-dynamics energy-transition models. Energy Policy 122, 260-272. https://doi.org/10.1016/j.enpol.2018.06.041

Saltelli, A., Bammer, G., Bruno, I., Charters, E., Fiore, M.D., Didier, E., Espeland, W.N., Kay, J., Piano, S.L., Mayo, D., Jr, R.P., Portaluri, T., Porter, T.M., Puy, A., Rafols, I., Ravetz, J.R., Reinert, E., Sarewitz, D., Stark, P.B., Stirling, A., Sluijs, J. van der, Vineis, P., 2020. Five ways to ensure that models serve society: a manifesto. Nature $582,482-484$. https://doi.org/10.1038/d41586-020-01812-9 
Samsó, R., de Blas, I., Perissi, I., Martelloni, G., Solé, J., 2020. Scenario analysis and sensitivity exploration of the MEDEAS Europe energy-economy-environment model. Energy Strategy Reviews 100582. https://doi.org/10.1016/j.esr.2020.100582

Samsó, R., Madurell, T., Solé, J., de Blas, I., Perissi, I., Martelloni, G., Bardi, U., Natalini, D., 2019. D5.2 Models statistics (Deliverable MEDEAS project (Private) No. D5.2). MEDEAS project, Barcelona, Spain.

Sanchez, L.F., Stern, D.I., 2016. Drivers of industrial and non-industrial greenhouse gas emissions. Ecological Economics 124, 17-24. https://doi.org/10.1016/j.ecolecon.2016.01.008

Sanz, A., Vega, P., Mateos, M., 2014. Las cuentas ecológicas del transporte en España. Libros en Acción, Madrid.

Sargent, R.G., 2013. Verification and validation of simulation models. J Simulation 7, 12-24. https://doi.org/10.1057/jos.2012.20

Sarofim, M.C., Reilly, J.M., 2011. Applications of integrated assessment modeling to climate change. Wiley Interdisciplinary Reviews: Climate Change 2, 27-44. https://doi.org/10.1002/wcc.93

Scheidel, A., Sorman, A.H., 2012. Energy transitions and the global land rush: Ultimate drivers and persistent consequences. Global Environmental Change, Global transformations, social metabolism and the dynamics of socio-environmental conflicts $22,588-595$. https://doi.org/10.1016/j.gloenvcha.2011.12.005

Schenk, N.J., Moll, H.C., 2007. The use of physical indicators for industrial energy demand scenarios. Ecological Economics, Ecological Economics of Coastal Disasters 63, 521535. https://doi.org/10.1016/j.ecolecon.2006.12.008

Schneider, M., Froggatt, A., 2017. The World Nuclear Industry Status Report 2017. Mycle Schneider Consulting Project, Paris, London, Washington DC.

Schneider, S., Lane, J., 2005. Integrated Assessment Modeling of Global Climate Change: Much Has Been Learned-Still a Long and Bumpy Road Ahead. Integrated Assessment 5.

Schneider, S.H., 1997. Integrated assessment modeling of global climate change: Transparent rational tool for policy making or opaque screen hiding value-laden assumptions? Environmental Modeling \& Assessment 2, 229-249. https://doi.org/10.1023/A:1019090117643

Schneider, S.H., Morton, L., 1981. The Primordial Bond Exploring Connections Between Man and Nature Through the Humanities and Sciences. Plenum Press, New York.

Schwaninger, M., Groesser, S., 2020. System Dynamics Modeling: Validation for Quality Assurance, in: Dangerfield, B. (Ed.), System Dynamics: Theory and Applications, Encyclopedia of Complexity and Systems Science Series. Springer US, New York, NY, pp. 119-138.

Schwanitz, V.J., 2013. Evaluating integrated assessment models of global climate change. Environmental Modelling \& Software 50, 120-131. https://doi.org/10.1016/j.envsoft.2013.09.005

Scott, V., Gilfillan, S., Markusson, N., Chalmers, H., Haszeldine, R.S., 2013. Last chance for carbon capture and storage. Nature Clim. Change 3, 105-111. https://doi.org/10.1038/nclimate1695

Scrieciu, S., Rezai, A., Mechler, R., 2013. On the economic foundations of green growth discourses: the case of climate change mitigation and macroeconomic dynamics in economic modeling. WENE 2, 251-268. https://doi.org/10.1002/wene.57

Searchinger, T., Heimlich, R., Houghton, R.A., Dong, F., Elobeid, A., Fabiosa, J., Tokgoz, S., Hayes, D., Yu, T.-H., 2008. Use of U.S. Croplands for Biofuels Increases Greenhouse Gases Through Emissions from Land-Use Change. Science 319, 1238-1240. https://doi.org/10.1126/science.1151861

Sers, M.R., Victor, P.A., 2018. The Energy-missions Trap. Ecological Economics 151, 10-21. https://doi.org/10.1016/j.ecolecon.2018.04.004 
Shanker, A., Stern, D.I., 2018. ENERGY AND THE DIRECTION OF GROWTH: WILL AUTONOMOUS ENERGY EFFICIENCY IMPROVEMENTS CONTINUE?

SHC, 2016. Solar Heat Worldwide. Markets and Contribution to the Energy Supply 2014. Solar Heating \& Cooling Programme IEA.

Siegel, L.S., Homer, J., Fiddaman, T., McCauley, S., Franck, T., Sawin, E., Jones, A.P., Sterman, J.D., 2020. EN-ROADS SIMULATOR REFERENCE GUIDE.

Sims, R., Schaeffer, R., Creutzig, F., Cruz-Núñez, X., D’Agosto, M., Dimitriu, D., Figueroa Meza, M.J., Fulton, L., Kobayashi, S., Lah, O., McKinnon, A., Newman, P., Ouyang, M., Schauer, J.J., Sperling, D., Tiwari, G., 2014. Transport. In: Climate Change 2014: Mitigation of Climate Change. Contribution of Working Group III to the Fifth Assessment Report of the Intergovernmental Panel on Climate Change. Cambridge University Press, Cambridge, United Kingdom and New York, NY, USA.

Skrúcaný, T., Kendra, M., Stopka, O., Milojević, S., Figlus, T., Csiszár, C., 2019. Impact of the Electric Mobility Implementation on the Greenhouse Gases Production in Central European Countries. Sustainability 11, 4948. https://doi.org/10.3390/su11184948

Smil, V., 2015. Power density: a key to understanding energy sources and uses. MIT Press.

Smil, V., 2010. Energy Transitions: History, Requirements, Prospects. Praeger, Santa Barbara, California, USA.

Smil, V., 2008. Energy in nature and society: general energetics of complex systems. MIT Press, Cambridge, Massachusetts, USA.

Smith, P., 2016. Soil carbon sequestration and biochar as negative emission technologies. Glob Change Biol 22, 1315-1324. https://doi.org/10.1111/gcb.13178

Smith, P., Gregory, P.J., Vuuren, D. van, Obersteiner, M., Havlík, P., Rounsevell, M., Woods, J., Stehfest, E., Bellarby, J., 2010. Competition for land. Philosophical Transactions of the Royal Society B: Biological Sciences 365, 2941-2957. https://doi.org/10.1098/rstb.2010.0127

Solé, J., Samsó, R., García-Ladona, E., García-Olivares, A., Ballabrera-Poy, J., Madurell, T., Turiel, A., Osychenko, O., Álvarez, D., Bardi, U., Baumann, M., Buchmann, K., Capellán-Pérez, Í., Černý, M., Carpintero, Ó., De Blas, I., De Castro, C., De Lathouwer, J.-D., Duce, C., Eggler, L., Enríquez, J.M., Falsini, S., Feng, K., Ferreras, N., Frechoso, F., Hubacek, K., Jones, A., Kaclíková, R., Kerschner, C., Kimmich, C., Lobejón, L.F., Lomas, P.L., Martelloni, G., Mediavilla, M., Miguel, L.J., Natalini, D., Nieto, J., Nikolaev, A., Parrado, G., Papagianni, S., Perissi, I., Ploiner, C., Radulov, L., Rodrigo, P., Sun, L., Theofilidi, M., 2020. Modelling the renewable transition: Scenarios and pathways for a decarbonized future using pymedeas, a new open-source energy systems model. Renewable and Sustainable Energy Reviews 132, 110105. https://doi.org/10.1016/j.rser.2020.110105

Solow, R.M., 1973. Is the End of the World at Hand? Challenge 16, 39-50.

https://doi.org/10.1080/05775132.1973.11469961

Sorrell, S., 2015. Reducing energy demand: A review of issues, challenges and approaches. Renewable and Sustainable Energy Reviews 47, 74-82. https://doi.org/10.1016/j.rser.2015.03.002

SSP db, 2016. SSP Database (Shared Socioeconomic Pathways) - Version 1.1 (October 2016). Available at: https://tntcat.iiasa.ac.at/SspDb.

Stanton, E.A., Ackerman, F., Kartha, S., 2009. Inside the integrated assessment models: Four issues in climate economics. Climate and Development 1, 166-184. https://doi.org/10.3763/cdev.2009.0015

Statistica, 2018a. Average selling price of new vehicles - U.S. 2018 [WWW Document]. Statista. URL https://www.statista.com/statistics/274927/new-vehicle-average-selling-price-inthe-united-states/ (accessed 11.22.19).

Statistica, 2018b. Average car and van occupancy England 2002-2018 [WWW Document]. Statista. URL https://www.statista.com/statistics/314719/average-car-and-vanoccupancy-in-england/ (accessed 11.21.19). 
Staub-Kaminski, I., Zimmer, A., Jakob, M., Marschinski, R., 2014. Climate policy in practice: a typology of obstacles and implications for integrated assessment modeling. Clim. Change Econ. 5, 1440004. https://doi.org/10.1142/S2010007814400041

Steffen, W., Richardson, K., Rockström, J., Cornell, S.E., Fetzer, I., Bennett, E.M., Biggs, R., Carpenter, S.R., Vries, W. de, Wit, C.A. de, Folke, C., Gerten, D., Heinke, J., Mace, G.M., Persson, L.M., Ramanathan, V., Reyers, B., Sörlin, S., 2015. Planetary boundaries: Guiding human development on a changing planet. Science 347, 1259855. https://doi.org/10.1126/science.1259855

Steffen, W., Rockström, J., Richardson, K., Lenton, T.M., Folke, C., Liverman, D., Summerhayes, C.P., Barnosky, A.D., Cornell, S.E., Crucifix, M., Donges, J.F., Fetzer, I., Lade, S.J., Scheffer, M., Winkelmann, R., Schellnhuber, H.J., 2018. Trajectories of the Earth System in the Anthropocene. PNAS 115, 8252-8259. https://doi.org/10.1073/pnas.1810141115

Stehfest, E., van Vuuren, D.P., Kram, T., Bouwman, L., 2014. Integrated Assessment of Global Environmental Change with IMAGE 3.0 Model description and policy applications. PBL Netherlands Environmental Assessment Agency, The Hague.

Sterman, J., Fiddaman, T., Franck, T., Jones, A., McCauley, S., Rice, P., Sawin, E., Siegel, L., 2012. Climate interactive: the C-ROADS climate policy model. System Dynamics Review 28, 295-305. https://doi.org/10.1002/sdr.1474

Sterman, J.D., 2012. Sustaining Sustainability: Creating a Systems Science in a Fragmented Academy and Polarized World, in: Weinstein, M.P., Turner, R.E. (Eds.), Sustainability Science. Springer New York, pp. 21-58.

Sterman, J.D., 2000. Business dynamics: systems thinking and modeling for a complex world. Stern, D.I., 2017. How accurate are energy intensity projections? Climatic Change 143, 537545. https://doi.org/10.1007/s10584-017-2003-3

Stern, D.I., 2012. Modeling international trends in energy efficiency. Energy Economics 34, 2200-2208. https://doi.org/10.1016/j.eneco.2012.03.009

Stern, D.I., 1997. Limits to substitution and irreversibility in production and consumption: A neoclassical interpretation of ecological economics. Ecological Economics 21, 197-215. https://doi.org/10.1016/S0921-8009(96)00103-6

Stern, N., 2013. The Structure of Economic Modeling of the Potential Impacts of Climate Change: Grafting Gross Underestimation of Risk onto Already Narrow Science Models. Journal of Economic Literature 51, 838-859. https://doi.org/10.1257/jel.51.3.838

Stiglitz, J.E., Sen, A., Fitoussi, J.-P., 2010. Report by the Commission on the Measurement of Economic Performance and Social Progress. Commission on the Measurement of Economic Performance and Social Progress, Paris (France).

Suganthi, L., Samuel, A.A., 2012. Energy models for demand forecasting-A review. Renewable and Sustainable Energy Reviews 16, 1223-1240. https://doi.org/10.1016/j.rser.2011.08.014

Sverdrup, H.U., Ragnarsdottir, K.V., 2014. Natural resources in planetay perspective.

Tainter, J., 1990. The Collapse of Complex Societies. Cambridge University Press.

T\&E, 2018. CNG and LNG for vehicles and ships - the facts. Transport \& Environment, Brussels (Belgium).

Tietge, U., Diaz, S., Mock, P., German, J., Bandivadekar, A., Ligterink, N.E., 2016. From Laboratory to Road. A 2016 update of official and real-world fuel consumption and $\mathrm{CO} 2$ values for passenger cars in Europe (White Paper). International Council on Clean Transportation Europe.

Timmer, M., Erumban, A.A., Gouma, R., Los, B., Temurshoev, U., de Vries, G.J., Arto, I., Genty, V.A.A., Neuwahl, F., Francois, J., others, 2012. The world input-output database (WIOD): contents, sources and methods. Institue for International and Development Economics. 
Tokimatsu, K., Wachtmeister, H., McLellan, B., Davidsson, S., Murakami, S., Höök, M., Yasuoka, R., Nishio, M., 2017. Energy modeling approach to the global energy-mineral nexus: A first look at metal requirements and the $2^{\circ} \mathrm{C}$ target. Applied Energy, Transformative Innovations for a Sustainable Future - Part II 207, 494-509. https://doi.org/10.1016/j.apenergy.2017.05.151

Tol, R.S.J., 2018. The Economic Impacts of Climate Change. Rev Environ Econ Policy 12, 4-25. https://doi.org/10.1093/reep/rex027

Tol, R.S.J., 2006. Integrated Assessment Modelling (Working Paper FNU-102). Research unit Sustainability and Global Change, Hamburg University.

Toll, M., 2018. An electric motorcycle for $\$ 1,995$ in the US - where do I sign up!? Electrek. URL https://electrek.co/2018/07/23/electric-motorcycle-for-2000-usd/ (accessed 11.21.19).

Trainer, F., 2007. Renewable energy cannot sustain a consumer society. Springer Science \& Business Media.

Trainer, T., 2013. Can the world run on renewable energy? A revised negative case. Humanomics 29, 88-104. https://doi.org/10.1108/08288661311319166

Transport \& Environment, 2020. Recharge EU trucks: time to act! A roadmap for electric truck charging infrastructure deployment.

Transport \& Environment, 2016. Dieselgate: Who? What? How? | Transport \& Environment [WWW Document]. URL https://www.transportenvironment.org/publications/dieselgate-who-what-how (accessed 3.12.20).

Turner, G., 2014. Global Collapse Imminent? (No. MSSI Research Paper No. 4). Melbourne Sustainable Society Institute, The University of Melbourne.

Turner, G.M., 2008. A comparison of The Limits to Growth with 30 years of reality. Global Environmental Change, Globalisation and Environmental Governance: Is Another World Possible? 18, 397-411. https://doi.org/10.1016/j.gloenvcha.2008.05.001

UCS, 1992. World Scientists' Warning to Humanity. Union of Concerned Scientist.

Ueckerdt, F., Pietzcker, R., Scholz, Y., Stetter, D., Giannousakis, A., Luderer, G., 2017. Decarbonizing global power supply under region-specific consideration of challenges and options of integrating variable renewables in the REMIND model. Energy Economics 64, 665-684. https://doi.org/10.1016/j.eneco.2016.05.012

Uehara, T., Nagase, Y., Wakeland, W., 2016. Integrating Economics and System Dynamics Approaches for Modelling an Ecological-Economic System. Systems Research and Behavioral Science 33, 515-531. https://doi.org/https://doi.org/10.1002/sres.2373

UN, 2020. World Population Prospects 2019, Online Edition. Rev. 1. [WWW Document]. Department of Economic and Social Affairs, Population Division (2019). URL https://population.un.org/wpp/Download/Standard/Population/ (accessed 1.8.21).

UN, 2019. United Nations Population Division | Department of Economic and Social Affairs [WWW Document]. URL https://www.un.org/en/development/desa/population/index.asp (accessed 7.2.19).

UN, 2015a. Marco de indicadores mundiales para los Objetivos de Desarrollo Sostenible y metas de la Agenda 2030 para el Desarrollo Sostenible. Naciones Unidas.

UN, 2015b. Report of the Inter-Agency and Expert Group on Sustainable Development Goal Indicators. United Nations. Economic and Social Council. Statistical Commission.

UN, 1998. KYOTO PROTOCOL TO THE UNITED NATIONS FRAMEWORK CONVENTION ON CLIMATE CHANGE. United Nations.

UN, 1987. Report of the World Commission on Environment and Development: Our Common Future. United Nations.

UN Environment, 2020. Perspectiva Mundial sobre la Diversidad Biológica 5. Secretaría del Convenio sobre la Diversidad Biológica, Montreal. 
UNEP, 2013. Environmental risks and challenges of anthropogenic metals flows and cycles. UNEP.

UNEP, 2011a. Recycling rates of metals. A status report. International Resource Panel. United Nations Environment Programme.

UNEP, 2011b. Decoupling natural resource use and environmental impacts from economic growth. United Nations Environment Programme.

UNFCCC, 2015. Paris Agreement. Paris (France).

Urban ebikes, 2019. Electric Bikes, Ebikes, Mopeds and Scooters For Sale in London [WWW Document]. Urban eBikes. URL https://urbanebikes.com/ (accessed 11.21.19).

US Department of Commerce, N., 2020. Global Monitoring Laboratory - Carbon Cycle Greenhouse Gases [WWW Document]. URL https://www.esrl.noaa.gov/gmd/ccgg/trends/ (accessed 1.8.21).

US EIA db, 2018. USA Energy Statistics (Database). US Energy Information Administration, http://www.eia.gov.

USGS, 2019. Mineral Commodity Summaries [WWW Document]. URL https://www.usgs.gov/centers/nmic/mineral-commodity-summaries (accessed 10.17.19).

USGS, 2015. Mineral Commodity Summaries 2015. United States Geological Survey, https://minerals.usgs.gov/minerals/pubs/mcs/.

Valero, A., Carpintero, Ó., Valero, A., Calvo, G., 2014. How to account for mineral depletion. The exergy and economic mineral balance of Spain as a case study. Ecological Indicators 46, 548-559. https://doi.org/10.1016/j.ecolind.2014.07.021

Valero, A., Valero, A., Calvo, G., Ortego, A., 2018. Material bottlenecks in the future development of green technologies. Renewable and Sustainable Energy Reviews 93, 178-200. https://doi.org/10.1016/j.rser.2018.05.041

van de Ven, D.-J., Capellan-Peréz, I., Arto, I., Cazcarro, I., de Castro, C., Patel, P., GonzalezEguino, M., 2021. The potential land requirements and related land use change emissions of solar energy. Scientific Reports 11, 1-12. https://doi.org/10.1038/s41598021-82042-5

van de Ven, D.-J., González-Eguino, M., Arto, I., 2018. The potential of behavioural change for climate change mitigation: a case study for the European Union. Mitig Adapt Strateg Glob Change 23, 853-886. https://doi.org/10.1007/s11027-017-9763-y

van den Berg, N.J., Hof, A.F., Akenji, L., Edelenbosch, O.Y., van Sluisveld, M.A.E., Timmer, V.J., van Vuuren, D.P., 2019. Improved modelling of lifestyle changes in Integrated Assessment Models: Cross-disciplinary insights from methodologies and theories. Energy Strategy Reviews 26, 100420. https://doi.org/10.1016/j.esr.2019.100420

van den Bergh, J.C.J.M., 2009. The GDP paradox. Journal of Economic Psychology 30, 117-135. https://doi.org/10.1016/j.joep.2008.12.001

van der Zwaan, B., Keppo, I., Johnsson, F., 2013. How to decarbonize the transport sector? Energy Policy 61, 562-573. https://doi.org/10.1016/j.enpol.2013.05.118

Van Mierlo, J., Maggetto, G., Lataire, P., 2006. Which energy source for road transport in the future? A comparison of battery, hybrid and fuel cell vehicles. Energy Conversion and Management, 12th International Conference on Emerging Nuclear Energy Systems 47, 2748-2760. https://doi.org/10.1016/j.enconman.2006.02.004

van Sluisveld, M.A.E., Hof, A.F., Carrara, S., Geels, F.W., Nilsson, M., Rogge, K., Turnheim, B., van Vuuren, D.P., 2020. Aligning integrated assessment modelling with socio-technical transition insights: An application to low-carbon energy scenario analysis in Europe. Technological Forecasting and Social Change 151, 119177. https://doi.org/10.1016/j.techfore.2017.10.024

van Sluisveld, M.A.E., Martínez, S.H., Daioglou, V., van Vuuren, D.P., 2016. Exploring the implications of lifestyle change in $2^{\circ} \mathrm{C}$ mitigation scenarios using the IMAGE integrated 
assessment model. Technological Forecasting and Social Change 102, 309-319. https://doi.org/10.1016/j.techfore.2015.08.013

van Vuuren, D.P., de Vries, B., Beusen, A., Heuberger, P.S.C., 2008. Conditional probabilistic estimates of 21st century greenhouse gas emissions based on the storylines of the IPCC-SRES scenarios. Global Environmental Change, Local evidence on vulnerabilities and adaptations to global environmental change 18, 635-654. https://doi.org/10.1016/j.gloenvcha.2008.06.001

van Vuuren, D.P., Edmonds, J., Kainuma, M., Riahi, K., Thomson, A., Hibbard, K., Hurtt, G.C., Kram, T., Krey, V., Lamarque, J.-F., Masui, T., Meinshausen, M., Nakicenovic, N., Smith, S.J., Rose, S.K., 2011. The representative concentration pathways: an overview. Climatic Change 109, 5-31. https://doi.org/10.1007/s10584-011-0148-z

van Vuuren, D.P., Kok, M.T.J., Girod, B., Lucas, P.L., de Vries, B., 2012. Scenarios in Global Environmental Assessments: Key characteristics and lessons for future use. Global Environmental Change 22, 884-895. https://doi.org/10.1016/j.gloenvcha.2012.06.001 van Vuuren, D.P., Stehfest, E., Gernaat, D.E.H.J., van den Berg, M., Bijl, D.L., de Boer, H.S., Daioglou, V., Doelman, J.C., Edelenbosch, O.Y., Harmsen, M., Hof, A.F., van Sluisveld, M.A.E., 2018. Alternative pathways to the $1.5^{\circ} \mathrm{C}$ target reduce the need for negative emission technologies. Nature Climate Change 8, 391-397. https://doi.org/10.1038/s41558-018-0119-8

Velasco-Fernández, R., Giampietro, M., Bukkens, S.G.F., 2018. Analyzing the energy performance of manufacturing across levels using the end-use matrix. Energy 161, 559-572. https://doi.org/10.1016/j.energy.2018.07.122

Voigt, S., De Cian, E., Schymura, M., Verdolini, E., 2014. Energy intensity developments in 40 major economies: Structural change or technology improvement? Energy Economics 41, 47-62. https://doi.org/10.1016/j.eneco.2013.10.015

Vrontisi, Z., Luderer, G., Saveyn, B., Keramidas, K., Lara, A.R., Baumstark, L., Bertram, C., Boer, H.S. de, Drouet, L., Fragkiadakis, K., Fricko, O., Fujimori, S., Guivarch, C., Kitous, A., Krey, V., Kriegler, E., Broin, E.Ó., Paroussos, L., Vuuren, D. van, 2018. Enhancing global climate policy ambition towards a $1.5 \backslash$ hspace $0.167 \mathrm{em}^{\circ} \mathrm{C}$ stabilization: a short-term multi-model assessment. Environ. Res. Lett. 13, 44039. https://doi.org/10.1088/17489326/aab53e

Wang, J., Feng, L., Tang, X., Bentley, Y., Höök, M., 2017. The implications of fossil fuel supply constraints on climate change projections: A supply-side analysis. Futures $86,58-72$. https://doi.org/10.1016/j.futures.2016.04.007

WBGU, 2009. Future Bioenergy and Sustainable Land Use. German Advisory Council on Global Change (WBGU).

Wei, C., Ni, J., Shen, M., 2009. Empirical Analysis of Provincial Energy Efficiency in China. China \& World Economy 17, 88-103. https://doi.org/10.1111/j.1749-124X.2009.01168.x

Weitzman, M.L., 2012. GHG Targets as Insurance Against Catastrophic Climate Damages. Journal of Public Economic Theory 14, 221-244. https://doi.org/10.1111/j.14679779.2011.01539.x

Weyant, J., 2017. Some Contributions of Integrated Assessment Models of Global Climate Change. Rev Environ Econ Policy 11, 115-137. https://doi.org/10.1093/reep/rew018

White, L.A., 1943. Energy and the Evolution of Culture. American Anthropologist 45, 335-356. https://doi.org/10.1525/aa.1943.45.3.02a00010

Wilson, C., Kriegler, E., van Vuuren, D.P., Guivarch, C., Frame, D., Krey, V., Osborn, T.J., Schwanitz, V.J., Thompson, E.L., 2017. Evaluating Process-Based Integrated Assessment Models of Climate Change Mitigation (IIASA Working Paper (WP-17-007)). IIASA, Laxenburg, Austria.

Wing, I.S., 2008. Explaining the declining energy intensity of the US economy. Resource and Energy Economics 30, 21-49. 
WITCH, 2020. WITCH documentation [WWW Document]. URL https://doc.witchmodel.org/ (accessed 9.16.20).

WMO, 2020. WMO Statement on the State of the Global Climate in 2019. World Meteorological Organization (WMO), Geneva, Switzerland.

World Bank, 2020. GDP, PPP (constant 2017 international \$) [WWW Document]. URL https://data.worldbank.org/indicator/NY.GDP.MKTP.PP.KD?end=2019\&start=1990 (accessed 1.8.21).

Wurlod, J.-D., Noailly, J., 2018. The impact of green innovation on energy intensity: An empirical analysis for 14 industrial sectors in OECD countries. Energy Economics 71, 47-61. https://doi.org/10.1016/j.eneco.2017.12.012

Wynes, S., Nicholas, K.A., 2017. The climate mitigation gap: education and government recommendations miss the most effective individual actions. Environ. Res. Lett. 12, 74024. https://doi.org/10.1088/1748-9326/aa7541

Wynes, S., Nicholas, K.A., Zhao, J., Donner, S.D., 2018. Measuring what works: quantifying greenhouse gas emission reductions of behavioural interventions to reduce driving, meat consumption, and household energy use. Environ. Res. Lett. 13, 113002. https://doi.org/10.1088/1748-9326/aae5d7

$\mathrm{Xu}, \mathrm{Y}$., Ramanathan, V., 2017. Well below $2{ }^{\circ} \mathrm{C}$ : Mitigation strategies for avoiding dangerous to catastrophic climate changes. PNAS 114, 10315-10323. https://doi.org/10.1073/pnas.1618481114

Yeh, S., Mishra, G.S., Fulton, L., Kyle, P., McCollum, D.L., Miller, J., Cazzola, P., Teter, J., 2017. Detailed assessment of global transport-energy models' structures and projections. Transportation Research Part D: Transport and Environment 55, 294-309. https://doi.org/10.1016/j.trd.2016.11.001

Zagamé, P., Boitier, B., Fougeyrollas, A., Mouël, P.L., Capros, P., Kouvaritakis, N., Bossier, F., Thierry, F., Melon, A., 2018. NEMESIS Model: Full description.

Zemp, M., Huss, M., Thibert, E., Eckert, N., McNabb, R., Huber, J., Barandun, M., Machguth, H., Nussbaumer, S.U., Gärtner-Roer, I., Thomson, L., Paul, F., Maussion, F., Kutuzov, S., Cogley, J.G., 2019. Global glacier mass changes and their contributions to sea-level rise from 1961 to 2016. Nature 568, 382-386. https://doi.org/10.1038/s41586-019-1071-0

Zenzey, E., 2013. Energy as a Master Resource, in: State of the World 2013: Is Sustainability Still Possible? Worldwatch Institute, Washington: Island Press, pp. 73-83.

Zhao, H., Burke, A., Zhu, L., 2013. Analysis of Class 8 Hybrid-Electric Truck Technologies Using Diesel, LNG, Electricity, and Hydrogen, as the Fuel for Various Applications 1-16. https://doi.org/10.1109/EVS.2013.6914957

zhiyi, Y., Xunmin, O., 2019. Life Cycle Analysis on Liquefied Natural Gas and Compressed Natural Gas in Heavy-duty Trucks with Methane Leakage Emphasized. Energy Procedia, Innovative Solutions for Energy Transitions 158, 3652-3657. https://doi.org/10.1016/j.egypro.2019.01.896

Zittel, W., 2012. Feasible Futures for the Common Good. Energy Transition. Paths in a Period of Increasing Resource Scarcities. Ludwig-Bölkow-Systemtechnik GmbH, Munich (Germany).

Zittel, W., Zerhusen, J., Zerta, M., 2013. Fossil and Nuclear Fuels - the Supply Outlook. Energy Watch Group.

Zu, C.-X., Li, H., 2011. Thermodynamic analysis on energy densities of batteries. Energy Environ. Sci. 4, 2614-2624. https://doi.org/10.1039/COEE00777C

Zuberi, M.J.S., Patel, M.K., 2017. Bottom-up analysis of energy efficiency improvement and $\mathrm{CO} 2$ emission reduction potentials in the Swiss cement industry. Journal of Cleaner Production 142, 4294-4309. https://doi.org/10.1016/j.jclepro.2016.11.178 